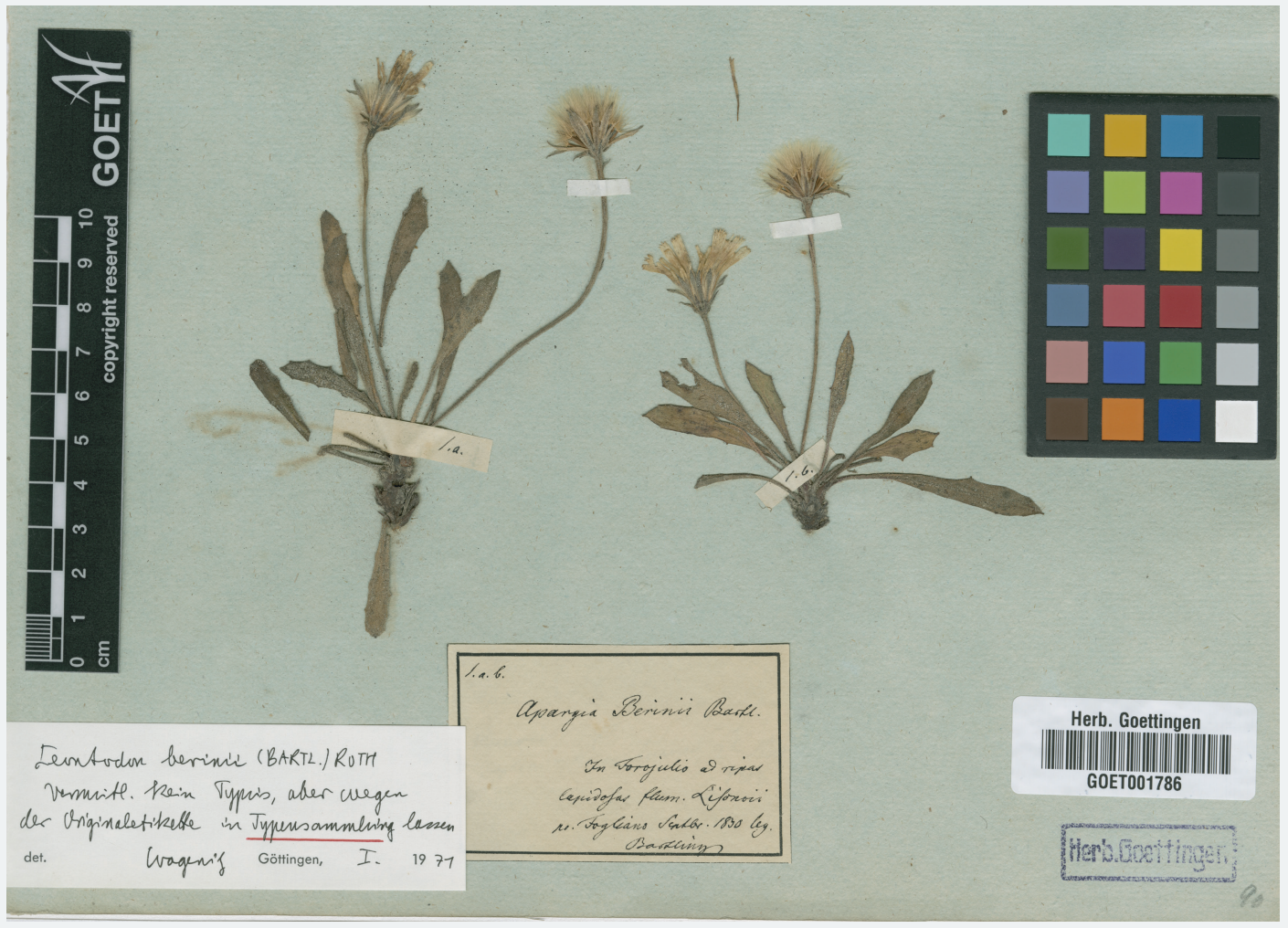

Frauke Johanna Ahrens

Das Herbarium Göttingen, 1832-1852

Akteure, Praktiken, Wissensformate 

Frauke Johanna Ahrens

Das Herbarium Göttingen, 1832-1852

Dieses Werk ist lizenziert unter einer

Creative Commons

Namensnennung - Weitergabe unter gleichen Bedingungen

4.0 International Lizenz.

() (1) ( 
erschienen als Band 13 in der Reihe „Göttinger Studien zur Kulturanthropologie/Europäischen Ethnologie“ im Universitätsverlag Göttingen 2020 
Frauke Johanna Ahrens

Das Herbarium Göttingen, 1832-1852

Akteure, Praktiken, Wissensformate

Göttinger Studien zur

Kulturanthropologie/Europäischen Ethnologie, Band 13

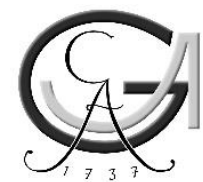

Universitätsverlag Göttingen 2020 


\section{Bibliographische Information der Deutschen Nationalbibliothek}

Die Deutsche Nationalbibliothek verzeichnet diese Publikation in der Deutschen Nationalbibliographie; detaillierte bibliographische Daten sind im Internet über $<$ http://dnb.dnb.de $>$ abrufbar.

„Göttinger Studien zur Kulturanthropologie/Europäischen Ethnologie“, herausgegeben von

Prof. Dr. Regina Bendix

E-Mail: rbendix@gwdg.de

Prof. Dr. Moritz Ege

E-Mail: mege@uni-goettingen.de

Prof. Dr. Sabine Hess

E-Mail: shess@uni-goettingen.de

Prof. Dr. Carola Lipp

E-Mail: Carola.Lipp@phil.uni-goettingen.de

Dr. Torsten Näser

E-Mail: tnaeser1@gwdg.de

Georg-August-Universität Göttingen

Institut für Kulturanthropologie/Europäische Ethnologie

Heinrich-Düker-Weg 14

37073 Göttingen

Anschrift der Autorin

Frauke Johanna Ahrens

E-Mail: frauke.ahrens@outlook.com

Dieses Buch ist auch als freie Onlineversion über die Homepage des Verlags sowie über den Göttinger Universitätskatalog (GUK) bei der Niedersächsischen Staats- und Universitätsbibliothek Göttingen (http://www.sub.uni-goettingen.de) erreichbar.

Es gelten die Lizenzbestimmungen der Onlineversion.

Satz und Layout: Frauke Johanna Ahrens

Umschlaggestaltung: Jutta Pabst

Titelabbildung: Herbarbeleg von Leontodon berinii (Bartl.) Roth, gesammelt von F. G. Bartling im September 1830 in Forojulio in der Nähe des italienischen Ortes Fogliano

Redipuglia, Herbarium Göttingen, GOET001786

(C) 2020 Universitätsverlag Göttingen

http://univerlag.uni-goettingen.de

ISBN: 978-3-86395-450-5

DOI: https://doi.org/10.17875/gup2020-1305

eISSN: 2512-7055 


\section{Danke}

Von der ersten Idee bis zur fertigen Arbeit haben mich viele Personen begleitet und unterstützt. Ihnen möchte ich an dieser Stelle meinen besonderen Dank aussprechen.

In erster Linie danke ich meinen beiden Betreuerinnen Prof. Dr. Regina Bendix und Prof. Dr. Margarete Vöhringer, die mich nicht allein inhaltlich betreut, sondern maßgeblich dazu beigetragen haben, dass meine Arbeit auch zur Publikation kommen konnte. Ihr Zuspruch hat mich durch den Forschungs- und Schreibprozess getragen und mir Mut für nachfolgende Zukunftsplanungen gemacht. Besonders für die finanzielle Unterstützung sei an dieser Stelle ihnen beiden und dem Institut für Kulturanthropologie/Europäische Ethnologie ganz herzlich gedankt.

Ohne das reichhaltige Quellenkorpus wäre meine Arbeit allerdings nicht möglich gewesen. Dazu gehören zuallererst die Bestände des Göttinger Herbariums. Dem Kurator der Sammlung Dr. Marc Appelhans danke ich für die Führungen durch die Sammlung, die Zeit, die er für meine vielen Fragen einräumte, sowie das entgegengebrachte Vertrauen. Der Austausch war mir eine große Hilfe und Freude. Auch dem Universitätsarchiv Göttingen, der Staats- und Universitätsbibliothek Göttingen sowie dem Stadtarchiv Göttingen danke ich für die freundliche Bereitstellung der Bestände.

Matthias Lund danke ich für seine inhaltlichen Anmerkungen, klugen Nachfragen und das detaillierte Lektorat. Vielen Dank für deine Geduld und Selbstlosigkeit.

Schließlich danke ich meiner Familie und meinen Freund*innen, die mich zur rechten Zeit immer zum Innehalten und Durchatmen zu bringen wussten. Danke für eure Liebe. 



\section{Inhaltsverzeichnis}

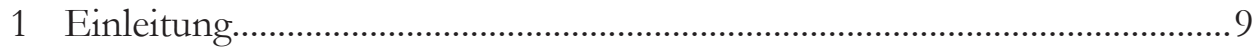

2 Eine historische Ethnografie und Praxeografie ............................................21

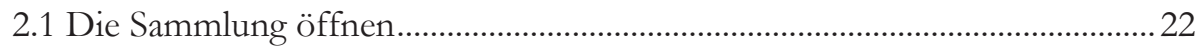

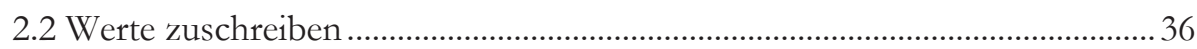

2.3 Pflanzenexemplare akquirieren........................................................................ 53

2.4 Mit Herbarbelegen forschen.............................................................................. 78

2.5 Mit Herbarbelegen lehren .................................................................................. 99

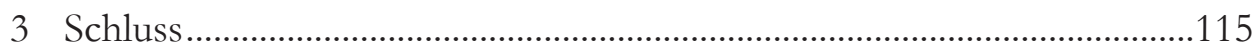

4 Quellen- und Literaturverzeichnis .............................................................121

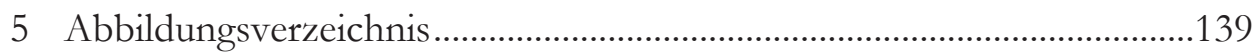





\section{$1 \quad$ Einleitung}

I. Fragestellung und Einführung in die Thematik

Kaum ein Objekt ist in den Sammlungen der Universität Göttingen so häufig vertreten wie das Präparat. Doch auch wenn viele dieser Wissensdinge heute noch in die akademische Arbeit eingebunden sind, die Bestände weiterhin anwachsen und für die Institute anhaltend Relevanz besitzen, wurde die Genese vieler naturwissenschaftlicher Sammlungen bisher kaum oder gar nicht beforscht. Dies gilt auch für das 1832 institutionalisierte Göttinger Herbarium der Abteilung Systematische Botanik des Albrecht-von-Haller-Instituts für Pflanzenwissenschaften. Auf der Grundlage von privaten Sammlungen, die zum Teil auch zu den Beständen des 1773 gegründeten Königlichen Academischen Museums gehörten, entstanden und seitdem stetig gewachsen, genießt das Göttinger Herbarium heute internationales Ansehen. Während sich die Sammlung durch Forschungsreisen der Wissenschaftler*innen ${ }^{1}$ des Instituts sowie Schenkungen weiter vergrößert, die Pflanzenexemplare für wissenschaftliche Untersuchungen zurate gezogen werden und ein

\footnotetext{
${ }^{1}$ Vorliegende Arbeit nutzt eine gendergerechte Sprache. Um historische Situationen nicht zu verfälschen und den Blick für ungleiche Verhältnisse zu schärfen, findet an einigen Stellen dennoch die maskuline Form Verwendung. Das betrifft besonders jene Passagen, die sich auf die ausschließlich männlichen Dozierenden und Studierenden des Göttinger Instituts in dem hier betrachteten Zeitraum beziehen. Ist allerdings von Personengruppen wie Botaniker*innen oder Wissenschaftler*innen die Rede, wird eine geschlechtsneutrale Sprache verwendet.
} 
Teil der Sammlung bereits in aufwendigen Digitalisierungsprojekten für die Zukunft gesichert wurde, ist ihre Geschichte bisher aber nur rudimentär behandelt worden.

Die vorliegende Arbeit fragt deshalb aus kulturanthropologischer Perspektive nach der wissens- und wissenschaftsgeschichtlichen Bedeutung des Göttinger Herbariums. Im Fokus der Untersuchung stehen die Herbarbelege und die mit ihnen in Verbindung stehenden Akteure und wissenskonstituierenden Praktiken im Zeitraum von 1832 bis 1852 . Vor dem Hintergrund einer historischen, objektfokussierten Ethnografie und Praxeologie soll aufgeschlüsselt werden, wie sich diese Praktiken und das wissenschaftliche Erkenntnisinteresse in der Etablierungsphase der Sammlung wechselseitig beeinflusst haben und sich schließlich auch in den Sammlungsobjekten niederschlugen. Inwiefern haben sich also die wissenskonstituierenden Praktiken in die Herbarbelege eingeschrieben? Und wo hat ein Wandel derselben zu einer Bedeutungsverschiebung der wissenschaftlichen Objekte geführt? Gefragt wird nach den Ordnungssystemen, auf die bei der Formatierung des Wissens zurückgegriffen wurde, die reproduziert oder neu geschrieben wurden. Welche Regeln der Wissensordnung und -verarbeitung sind auszumachen? Welches Wissen wurde verarbeitet und welches wiederum ausgeschlossen? Welche Regeln und Funktionsweisen bestimmten die Erstellung von Herbarbelegen im Göttinger Herbarium? Und welche Akteure waren an diesen wissenskonstituierenden Praktiken beteiligt? Von Relevanz sind folglich Fragen nach wiederkehrenden Ordnungsprinzipien, aber auch nach der Transformation und dem Zerfall derselben. Konkret in den Blick genommen werden die Sammlungspraxis des Herbariums, die verschiedenen Sammelstrategien, die Aufnahmekriterien für Pflanzen und die Art und Weise ihrer Aufbewahrung sowie ihre Bedeutung und Nutzung in Forschung und Lehre: Auf welchen Wegen kamen die Pflanzen in das Göttinger Herbarium und wie wurden sie zu Präparaten? Was zeichnete einen Herbarbeleg aus? Welchen Ordnungsprinzipien unterlag das Herbarium und in welchen Räumlichkeiten und Mobiliaren wurden die Präparate aufbewahrt? Inwiefern waren die Objekte schließlich in die Lehr- und Forschungspraxis eingebunden?

\section{Forschungsstand}

Mit diesen Fragestellungen wird vor allem an die Untersuchungen und theoretischen Überlegungen dreier Forschungsfelder angeschlossen: der wissens- und wissenschaftsgeschichtlichen Forschung, der Material Culture Studies sowie der historisch-quellenbasierten Forschung. In allen Bereichen hat die Kulturanthropologie wichtige Beiträge geleistet, die die vorliegende Arbeit aufgreifen möchte, wenngleich die Fokussierung auf eine naturwissenschaftliche Universitätssammlung sowie die internationale und interdisziplinäre Ausrichtung aller drei Themenfelder den Einbezug von Untersuchungen über die Fachgrenze hinaus erforderlich machen. Denn während das Museum und sein Vorgänger, die 
frühneuzeitliche Wunderkammer, zentraler Bestandteil von Untersuchungen materieller Kultur im Fach sind, wurden naturwissenschaftliche und genauer universitäre naturwissenschaftliche Sammlungen hier noch nicht verstärkt in den Blick genommen. Die kulturanthropologische Wissenschafts-, Medizin- und Technikforschung arbeitet zwar immer wieder über andere und mit anderen Disziplinen, doch richtete sie ihr Augenmerk bisher ebenso wenig auf diese Thematik.

Naturwissenschaftliche ${ }^{2}$ Wissens- und Wissenschaftsforschung wird in erster Linie von der Wissenschaftsgeschichte selbst sowie von Teilen der deutschen Geschichtswissenschaft betrieben. Die zentralen Publikationen der letzten zwanzig Jahre widmen sich dabei dem Wissens- und Objekttransfer in Hinblick auf Korrespondenzen und Netzwerke, aber auch im Kontext von weltweitem, kolonialem Handel. ${ }^{3}$ Gerade die anknüpfenden Arbeiten, die die Auswirkungen dieser Austauschbeziehungen auf die Standardisierung, Systematisierung, Ordnung und Anordnung von Wissensbeständen und (Sammlungs-)Objekten thematisieren, waren für diese Arbeit in besonderer Weise anschlussfähig. ${ }^{4}$ Ebenso wurden diejenigen Veröffentlichungen von Seiten der naturwissenschaftlichen Wissensforschung berücksichtigt, die den Zusammenhang von Wissens- und Objekterzeugung und damit die materielle Verfasstheit von Wissen und Erkenntnis herausstellen. ${ }^{5}$

Obwohl ein Großteil dieser Forschungen auch naturwissenschaftliche Sammlungsobjekte und Sammlungspraktiken thematisiert, findet die Fokussierung auf die Vernetzung und den Transfer von Wissen und Wissensbeständen häufig unter Vernachlässigung einer umfassenden Untersuchung spezifischer Sammlungsbestände statt. Sofern diese in den Blick genommen werden, geschieht dies vor allem im Zusammenhang mit bekannten Forschungspersönlichkeiten ${ }^{6}$, der Geschichte der Wunderkammern und Naturaliensammlungen ${ }^{7}$ sowie der

2 An dieser Stelle sei darauf hingewiesen, dass eine Trennschärfe zwischen den Begriffen Naturgeschichte, Naturwissenschaft und Botanik erst im Laufe des 19. Jahrhunderts möglich wurde. Deshalb wird bewusst nicht von botanischer Wissensforschung gesprochen, da die Botanik als eigenständige Disziplin erst im Laufe des 19. Jahrhunderts institutionalisiert worden ist. Dies spiegelt sich auch im Quellenmaterial wider, wo die Begriffe ,Naturgeschichte', ,Botanik' und ,Naturwissenschaft' sowie analog dazu ,Naturforscher' oder ,Botaniker' synonym Verwendung finden. Vgl. Jahn 1998, S. 306-308; Lefèvre 2016; Phillips 2012, S. 1-10.

3 Vgl. Bleichmar \& Mancall 2011; Habermas \& Przyrembel 2013; Miller \& Reill 1996;

Müller-Wille 1999; Müller-Wille 2005; Müller-Wille 2008; Schiebinger 2004; Schiebinger 2005; Siemer 2000; Vogel 2013.

${ }^{4}$ Vgl. Bonneuil 2002; Bourgouet et al. 2002; Daston 1999; Daston 2001; Daston 2004; Förschler \& Mariss 2017; Müller-Wille 1999; Müller-Wille 2017a; Müller-Wille 2017b; te Heesen 2001.

5 Vgl. Becker \& Clark 2001; Beretta 2005; Charmantier \& Müller-Wille 2014; Daston \& Galison 2007; Müller-Wille 2001; Müller-Wille \& Charmantier 2012; Smith et al. 2014; te Heesen \& Spary 2001; Yale 2016.

${ }^{6}$ Vgl. Napolitani 2018; te Heesen 2000; te Heesen 2005.

${ }^{7}$ Vgl. Dolezel et al. 2018. 
Herausbildung von Museen oder allgemeiner gesprochen der Präsentation und dem Ausstellen von Objekten ${ }^{8}$. Das Augenmerk liegt vornehmlich auf der Frühen Neuzeit, während das 19. Jahrhundert zumeist in einen Kontext mit Prozessen der Wissenschaftspopularisierung und dem Wechselverhältnis von Naturwissenschaft und Öffentlichkeit gesetzt wird. ${ }^{9}$ Die daran anzuschließende Frage nach den Rückwirkungsprozessen dieser Entwicklungen auf Universitätssammlungen sowie der gleichzeitig stattfindenden Ausdifferenzierung der Disziplinen blieb aber bisher weitestgehend unbeantwortet. ${ }^{10} \mathrm{Zu}$ einzelnen naturwissenschaftlichen Sammlungen geschweige denn Herbarien gibt es deshalb kaum einschlägige Literatur. ${ }^{11}$ Publikationen zu spezifischen universitären Sammlungen in Deutschland - darunter auch botanische Sammlungen - wurden in den letzten zehn Jahren vor allem im Rahmen von Begleitbänden zu Ausstellungsprojekten von den Hochschulen selbst veröffentlicht. ${ }^{12}$ Flankiert wird diese Literatur von vereinzelten Veröffentlichungen, die sich gezielt dem Herbarium widmen, aber aus Lehrforschungsprojekten hervorgingen. ${ }^{13}$ Für das Göttinger Herbarium besitzen besonders die Veröffentlichungen des ehemaligen Göttinger Botanikers Gerhard Wagenitz Relevanz, der sich als erster und einziger Wissenschaftler der Geschichte der Sammlung intensiv widmete. ${ }^{14}$ Seine Beiträge zur

8 Vgl. Kretschmann 2006; Pomian 1994; Pomian 1998; Savoy 2015; te Heesen \& Vöhringer 2014.

9 Vgl. Beretta 2005; Daum 1998; Daum 2002; Nyhart 2002; Scheidegger 2017.

${ }^{10}$ Dieses Defizit in der universitären Sammlungsforschung beobachtet auch Cornelia Weber in ihrem Beitrag zu Universitätssammlungen und -museen als Quellen frühneuzeitlicher Universitätsgeschichte: „Somit stellt sich die Frage, ob die Universität Göttingen auch beim Institutionalisierungsprozess von akademischen Sammlungen und damit bei der Modernisierung von Wissenschaft und Ausbildung eine Vorreiterrolle gespielt hat. Die bisher vorliegenden Daten unterstützen diese These; um dies zu belegen, sind jedoch weitere, detaillierte Untersuchungen notwendig." Weber 2011, S. 101.

11 Literatur über botanische Sammlungen behandelt vordergründig botanische Gärten sowie botanische Bücher und Illustrationen, während das Herbarium dahinter zurücktritt. Vgl. Cunningham 1996; Desmond 1995; Gottfried Wilhelm Leibniz-Bibliothek 2016; Isphording 2008; Nissen 1966; Outram 1996; Winnerling 2014.

12 Zentrale Beispiele hierfür sind die Ausstellung Theatrum naturae et artis - Theater der Natur und Kunst. Wunderkammern des Wissens aus dem Jahr 2000, die Ausstellung 38 Dinge - Schätze aus den naturund kulturwissenschaftlichen Sammlungen der Universität Tübingen von 2006, die Ausstellung Ausgepackt. Die Sammlungen der Universität Erlangen-Nürnberg aus dem Jahr 2007, die Ausstellung Weltwissen. 300 Jahre Wissenschaften in Berlin von 2010, der Sammelband Wissen sammeln. Die digitalisierten Schätze der Universität Greifswald. Sammlungsobjekte der Botanik, Zoologie sowie Ur- und Frühgeschichte sowie die 2018 veröffentlichte Publikation Wertsachen: Die Sammlungen der Johannes GutenbergUniversität Mainz: Vgl. Internetauftritt der Ausstellung 38 Dinge - Schätze aus den natur- und kulturwissenschaftlichen Sammlungen der Universität Tübingen; Andraschke \& Ruisinger 2007; Bredekamp et al. 2000; Dahlenburg 2011; Hennig \& Andraschke 2010; Hierholzer 2018. Ein entsprechender Band zu den Göttinger Sammlungen erschien 2015. Vgl. Georg-AugustUniversität Göttingen 2015.

13 Vgl. Müller \& Victor 2016; te Heesen et al. 2013.

14 Wagenitz' Beitrag zu einer wissens-/wissenschaftsgeschichtlichen Forschung zum Göttinger Herbarium ist besonders deshalb nicht zu unterschätzen, da er einen Index collectorum principalium berbarii Gottingensis anlegte, ein erstes Verzeichnis der für die Sammlung relevanten 
Sammlungsgeschichte bleiben allerdings auf die großen Erzählstränge fokussiert und sind als ideen- und personengeschichtliche Perspektivierungen einzustufen. Obwohl seitens der Botanik die Bedeutung und Relevanz von Herbarien vermehrt herausgestellt worden sind, wurden bisher keine wissensgeschichtlichen Untersuchungen diesbezüglich angestellt. ${ }^{15}$

Indem diese Arbeit ganz dezidiert einen konkreten Standort und eine einzelne (Universitäts-)Sammlung in einem Zeitraum von zwei Jahrzehnten fokussiert, setzt sie neue Akzente: Sie schreibt keine Erzählung zentraler Forschungspersönlichkeiten, hebt nicht besonders wertvolle Objekte der Sammlung hervor oder folgt herausragenden wissenschaftlichen Ideen und Erkenntnissen. Vielmehr wird - die Tendenzen der bisherigen richtungsweisenden Studien naturwissenschaftlicher Wissensforschung mitberücksichtigend - nach der Sammlungsgenese und der materiellen Bedingtheit von Wissen(schaft) am Beispiel einer einzigen Sammlung gefragt. Die kulturanthropologische Forschung materieller Kultur wird damit um die Auseinandersetzung mit naturwissenschaftlichen Objekten erweitert, während der ethnografische Zugang wiederum eine methodische Ergänzung bisheriger Projekte auf dem Gebiet der naturwissenschaftlichen Wissensforschung darstellt.

\section{Theorie-Bezug}

Nicht nur thematisch, sondern auch theoretisch bewegt sich die Arbeit in einem vielschichtigen Kräftefeld. Mit der Fokussierung auf Präparate einer naturwissenschaftlichen Universitätssammlung greift sie die an den material culture turn und material turn der 1980er Jahre anschließende Renaissance der Universitätssammlungen seit der Jahrtausendwende auf. Im Zuge des Paradigmenwechsels in den Geistes- und Sozialwissenschaften erfuhren physische Objekte in der neueren Wissens- und Wissenschaftsforschung gegenüber den zuvor privilegierten Diskursen, Ideen und Texten eine Aufwertung. ${ }^{16}$ Es wurde nicht mehr nur nach Erkenntnismöglichkeiten oder deren Konstruktionsweise gefragt, sondern die Ontologie, also das Seiende, in den Vordergrund gerückt. Diese in Abgrenzung und als Gegenpol zum linguistic und cultural turn entstandene antisemiotische Tendenzwende führte auch zu einer Stärkung universitärer Sammlungen. ${ }^{17}$

Sammler*innen, sowie einen Band über die Göttinger Biolog*innen veröffentlichte.

Vgl. Wagenitz 1982; Wagenitz 1988.

15 Vgl. Funk 2003; Hurka \& Neuffer 2011; Nualart et al. 2017.

16 Vgl. Eggert \& Samida 2016; Hahn 2016; Reckwitz 2014.

17 Vgl. te Heesen 2008. Die in den beiden letzten Jahrzehnten zunehmende Thematisierung von und Auseinandersetzung mit Universitätssammlungen äußert sich beispielsweise nicht nur in der weltweiten Etablierung der Unterorganisation UMAC (University Museums and Collections) des internationalen Museumsrates ICOM, die sich dezidiert mit den Belangen der Universitätssammlungen und -museen auseinandersetzt, oder auf Bundesebene in der Einrichtung einer umfassenden Datenbank zu allen deutschen Universitätssammlungen und der Koordinierungsstelle für wissenschaftliche Universitätssammlungen in Deutschland. Auch lokal macht sich 
Auch wenn für die Kulturanthropologie, die seit ihren Anfängen intensiv Sachkulturforschung betreibt, kein solcher Umbruch zu konstatieren ist, hat der theoretische Output sich doch erheblich im Fach niedergeschlagen. Gudrun Königs und Zuzanna Papierz' Vorschlag für eine qualitative Dinganalyse bietet beispielsweise eine eindrückliche Rückübersetzung ${ }^{18}$ neuer Impulse in die Fachdisziplin und ihren Umgang mit Objekten. ${ }^{19}$ Doch nicht nur auf die Sachkultur/Material Culture-Forschung, sondern auch auf die kulturanthropologische Wissenschafts-, Medizin- und Technikforschung haben die neuen Materialismen Einfluss genommen. Als Reaktion auf die Prägung des Alltags von Gegenwartskulturen durch Technik, Wissenschaft und Medizin und die Kombination sowie Verknüpfung technischer und menschlicher Möglichkeiten in immer mehr Alltagsbereichen globalisierter Gesellschaften, hat sie sich in den letzten Jahren zu einem wichtigen Bereich der Kulturanthropologie/Europäischen Ethnologie herausgebildet. ${ }^{20}$ Auch dort zu verortende theoretische Überlegungen werden bei einer wissensgeschichtlichen Auseinandersetzung mit naturwissenschaftlichen Präparaten wichtig. Besonders die Zentralität praxistheoretischer Konzepte innerhalb der Science and Technology Studies (STS) möchte vorliegendes Forschungsvorhaben aufgreifen. So betrachten die STS Wissen und Lernprozesse nicht als primär geistige Prozesse, sondern vielmehr als verkörperte, relationale und kontextuelle Praktiken. ${ }^{21}$ Gerade die symmetrische Betrachtung menschlicher und nichtmenschlicher Akteure, die den STS inhärent ist, macht sie besonders anschlussfähig. So sollen die Herbarbelege selbst als strukturierende und mitprägende Akteure botanischer Wissensfelder und Wissenspraktiken verstanden werden, die den Wissensbetrieb maßgeblich mitbeeinflussen. In diesem Sinne verpflichtet sich die Arbeit auch den grundlegenden Prinzipien der AkteurNetzwerk-Theorie und folgt vor allem auch Bruno Latours Überlegungen zu wissenschaftlichen Objekten. ${ }^{22}$

Begrifflich wird das vom DFG-Forschungsverbund Volkskundliches Wissen und gesellschaftlicher Wissenstransfer: zur Produktion kultureller Wissensformate im 20. Jahrbundert entwickelte theoretische Instrumentarium aufgegriffen. ${ }^{23}$ Dieses umfasst die Begriffe Wissensmilieu, Wissensraum, Wissensformat und Wissenstransfer und knüpft an eine erweiterte Perspektive der Wissenschaftsforschung an, die diese immer auch in einem engen Zusammenhang mit Wissensgeschichte betrachtet. Besonders die hinter den Begriffen Wissensmilieu und Wissensformat

diese Entwicklung in Göttingen durch die Gründung der Zentralen Kustodie und die Planungen für das zukünftige Wissensmuseum ,Forum Wissen' bemerkbar.

18 Vgl. Bachmann-Medick 2010.

19 Vgl. König 2004; König \& Papierz 2013.

${ }^{20}$ Vgl. Knecht 2013; Niewöhner et al. 2012.

21 Vgl. Knecht 2013, S 81.

22 Vgl. Latour 1997; Latour 2014.

23 Vgl. Boie et al. 2009; Davidovic-Walther et al. 2009; Dietzsch et al. 2009. 
stehenden Konzepte sind dabei auch für das vorliegende Forschungsvorhaben zentral. Verstanden als heuristisches Konzept meint der Begriff Wissensmilieu

„einen relativ homogenen, intern aber differenzierten Interaktionszusammenhang mit erhöhter Binnenkommunikation, der zugleich durch ein zumindest implizites Milieu-Wissen um gemeinsame Praxisformen geprägt ist. “24

Er soll für das Göttinger Herbarium mit seinen spezifischen institutionellen wie personellen Verbindungen und Netzwerken genutzt werden. Mit Hilfe des Begriffs des Wissensmilieus kann Wissen also als regional geordnet verstanden und eine Begrenzung des zu betrachtenden Feldes begründet werden. Der Begriff Wissensformat hingegen

„lenkt den Blick auf die tradierten Regeln, in denen Wissen erhoben, geformt und weitergegeben wird, auf den sinnlich-ästhetischen und sachkulturellen Charakter sowie auf die Prozesshaftigkeit der Wissensgenerierung und -weitergabe.“25

Er verdeutlicht, dass Informationen nie auf zufällige oder natürliche Weise geordnet werden, sondern in Anordnungen, Zurichtungen und Erscheinungen auftreten, die einer Praxis - der Formatierung - entspringen, die wiederum wissenschaftlich plausibel und gesellschaftlich gebunden ist. Indem Wissen in ein bestimmtes Format gebracht wird, erhält es also seine Gültigkeit. Dabei schließt das Konzept des Wissensformats die Verbindung zu außerwissenschaftlichen Kontexten und Akteur*innen nicht aus. Vielmehr lässt sich mit den Begriffen Format und Formatierung der gesamte Prozess wissenschaftlichen Arbeitens fassen, in dem auch unterschiedliche Öffentlichkeiten ${ }^{26}$ Beteiligung finden. Damit wird sich dezidiert wissenschaftsgeschichtlichen Diskursen angeschlossen, wie sie beispielsweise von Mitchel Ash, Philipp Sarasin, Arne Schirrmacher oder Andreas Daum geführt werden. ${ }^{27}$ Diese betrachten Wissenschaftsgeschichte vor allem in Form von Austauschprozessen, die immer auch in Verbindung mit verschiedenen Öffentlichkeiten stehen. Einem einseitigen Verständnis der Wissensgenerierung und Wissensvermittlung soll entgegengewirkt und vermehrt auch Rückwirkungseffekte zwischen Wissensproduzent*innen, Kommunikator*innen

${ }^{24}$ Boie et al. 2009, S. 186.

${ }^{25}$ Ebd., S. 189.

${ }^{26}$ Genauso wie die Nutzung des Terminus, Wissensgeschichte' anstelle von, Wissenschaftsgeschichte' verfolgt der Begriff, Öffentlichkeiten` die Absicht, von einer einfachen Gegenüberstellung von Wissenschaft auf der einen Seite und Öffentlichkeit auf der anderen Seite abzusehen. Arne Schirrmacher schlägt aus diesem Grund beispielsweise ein gestuftes Modell für verschiedene Wissenschaftsöffentlichkeiten vor, das neben den Polen ,Fachwissenschaft ${ }^{`}$ und ,breite Öffentlichkeiten' noch zusätzliche Abstufungen umfasst. Dadurch grenzt er sich von einem diffusionistischen Verständnis von Wissenschaft ab und betont stattdessen das häufig auch asymmetrische Wechselverhältnis zwischen Wissenschaft und Öffentlichkeit. Vgl. Schirrmacher 2008.

27 Vgl. Ash 2000; Ash 2007; Daum 1998; Daum 2002; Nikolow \& Schirrmacher 2007; Sarasin 2011; Schirrmacher 2008. 
und Öffentlichkeiten in den Blick genommen werden. Damit wird der Tatsache Rechnung getragen, dass Wissen stets auch in einem kollektiven Prozess generiert wird und mit vielschichtigen Wechselverhältnissen in Verbindung steht.

IV. Methodologische Erwägungen und empirischer Untersuchungsgegenstand

Methodisch ordnet sich das Forschungsvorhaben der historischen Ethnografie zu, wie sie besonders von Michaela Fenske, aber auch von anderen Kulturanthropolog*innen im Fach vertreten wird. ${ }^{28}$ Historische Forschung in der Kulturanthropologie/Europäischen Ethnologie wird dabei in Erweiterung und Vertiefung der bereits bestehenden theoretischen und methodischen Vorgehensweisen - der Historischen Anthropologie sowie der Mikrogeschichte - verstanden. Vergangenheits- und Gegenwartsforschung werden in der Historischen Ethnografie nicht in Abgrenzung zueinander gestellt, sondern in Bezug zueinander gesetzt und ein stärkerer theoretischer und methodischer Austausch befürwortet. Den Anmerkungen und Überlegungen Jens Wietschorkes folgend möchte die vorliegende Arbeit allerdings nicht die Verbindung zwischen Feldforschung und dem historisch-archivalischen Forschungsprozess betonen. ${ }^{29}$ Stattdessen wird das Ziel verfolgt, „die Stärken ethnografischen Denkens und Arbeitens auch für die historische Kulturanalyse zu operationalisieren“30. Damit schließt sich die Arbeit der Position Wietschorkes an, der zwar weniger ein methodologisches denn epistemologisches Verständnis von historischer Ethnografie vertritt, aber dennoch ihren heuristischen Wert sowie ihre Bedeutung in Hinblick auf die Schärfung des methodischen Instrumentariums herausstellt. ${ }^{31}$

Für eine Forschung über das Göttinger Herbarium bedeutet dies in der Folge, dass neben der historischen Quellenkritik und der qualitativ-hermeneutischen Textinterpretation auch Impulse aus der neuen Wissensforschung und der ethnografischen Gegenwartsforschung aufgenommen werden. Es werden nicht allein aktuelle dinganalytische Verfahren des Faches für die Arbeit fruchtbar gemacht oder in Anlehnung an die STS und historische wissens- und wissenschaftsgeschichtliche Forschung die Praxeologie ${ }^{32}$ als Zugangsweise befürwortet, sondern die ethnografische Erkenntnisweise insgesamt auf die Bearbeitung historischer Quellen übertragen. Damit ist Historische Ethnografie keine Methode an sich, bedingt aber die Herausbildung des Forschungsgegenstands sowie den darauf gerichteten Blick:

\footnotetext{
28 Vgl. Fenske 2006; Fenske 2013; Imeri \& Schneider 2013; Scholze-Irrlitz 2013.

${ }^{29} \mathrm{Vgl}$. Wietschorke 2013, S. 212.

30 Ebd., S. 210.

31 Vgl. Ebd., S. 211 f.

32 Vgl. Reckwitz 2003. Da Historische Ethnografie eben keine disziplinäre Abgrenzung meint, wird an dieser Stelle auch Bezug auf Ansätze der historischen Praxeologie genommen. Vgl. Elias et al. 2014; Freist 2015; Haasis \& Rieske 2015.
} 
„Übertragen wird dabei die Art und Weise, den Forschungsgegenstand zu konstituieren und zu kontextualisieren, das Forschungsfeld kleinräumig abzustecken, den Blick für das scheinbar Unbedeutende zu öffnen, den Fokus scharf zu stellen, mit der Dynamik von Nähe und Distanz zu spielen, die ,agency ${ }^{6}$ der Akteure ins Zentrum zu stellen und Handlungslogiken herauszuarbeiten. [...] Sie enthält auch [...] eine implizite Aufforderung, disparate Quellensorten heranzuziehen [...]. Diese Definition von Historischer Ethnografie betont das heuristische Moment: die Art und Weise, historisches Quellenmaterial in seiner Vielstimmigkeit praxeologisch aufzuschlüsseln.“33

Im Sinne eines solchen Verständnisses kulturanthropologischen historischen Arbeitens erfolgt die Herausbildung des empirischen Untersuchungsgegenstandes und der Datengrundlage. Dabei bedingen das der Arbeit zugrundeliegende Quellenkorpus sowie der gewählte Zeitraum sich gegenseitig: 1832 ist das Gründungsjahr des Herbariums und 1852 kehrte die Sammlung als etablierte Institution in den botanischen Garten zurück, wo sie von ihrer Abgabe durch das Academische Museum im Jahr 1792 an bis zur Gründung in der Dienstwohnung des Direktors aufbewahrt worden war. Diese ersten zwei Jahrzehnte werden als Etablierungsphase der Sammlung und damit als eine Verdichtung verstanden, die ein Herausarbeiten der wissens- und wissenschaftsgeschichtlichen Bedeutung der Sammlung begünstigt. Diese Annahme spiegelte sich auch eindeutig in der archivalischen Überlieferung wider: Sehr verdichtet liegen für den Zeitraum von 1832 bis 1852 Dokumente vor, die das Herbarium betreffen.

Berücksichtigt wurden die Bestände dreier Institutionen: des Göttinger Herbariums, des Universitätsarchivs Göttingen sowie der Niedersächsischen Staats- und Universitätsbibliothek Göttingen. Ergänzend dazu wurden Gespräche mit dem Kustos des Herbariums geführt, der schließlich auch den Zutritt zur Sammlung ermöglichte. ${ }^{34}$ Die Fokussierung auf Herbarbelege und die sie produzierenden wissenskonstituierenden Praktiken führte schließlich zu einem sehr vielschichtigen Quellenkorpus bestehend aus Verwaltungsakten, Nachlassüberlieferungen, Publikationen der Botaniker*innen sowie den Sammlungsobjekten selbst.

Den umfassendsten Teil dieses Korpus bildeten die Bestände des Universitätsarchivs. Besonders die Akten des Universitätskuratoriums, der staatlichen Aufsichtsbehörde der Universität, aber auch die der akademischen Selbstverwaltung, des Sekretariats, bildeten eine wichtige Grundlage. Gesichtet und ausgewertet wurden die Archivalien zur Gründung des Herbariums, die

\footnotetext{
33 Wietschorke 2013, S. 210 f.

${ }^{34}$ Das Göttinger Herbarium steht Forschenden zwar grundsätzlich zur Nutzung offen, doch ist zuvor eine Anmeldung erforderlich. Wissenschaftler*innen und Institutionen können für ihre Untersuchungen darüber hinaus auch Herbarbelege ausleihen.
} 
Rechnungen und deren Abnahme sowie Revision ${ }^{35}$, die Personalakten der zentralen Göttinger Botaniker*innen ${ }^{36}$, Gebäudeakten sowie die Regelungen zur Unterbringung des Herbariums und Akten zu der Assistentenstelle der Sammlung. Auch Archivalien über die Nachlassverwaltung zweier Privatsammlungen, die Verhandlungen über eine Forschungsreise sowie der Ankauf einer Sammlung wurden herangezogen. Aus dem historischen Bestand der Niedersächsischen Staats- und Universitätsbibliothek wurden besonders die Nachlässe der Botaniker*innen, die Nachschriften zu den botanischen Vorlesungen, die Vorlesungsverzeichnisse und einige der Publikationen der in Göttingen wirkenden Botaniker mitberücksichtigt. Im Herbarium wurden neben den Präparaten auch die im Büro des Kustoden aufbewahrten Archivalien in die Untersuchung miteinbezogen. Dazu gehören in erster Linie der Akzessionskatalog, ein Inventarium sowie die Rechnungen über Einnahmen und Ausgaben des Herbariums.

Trotz einer insgesamt dichten Quellenlage sind auch Leerstellen in der Überlieferung zu verzeichnen. Diese betreffen besonders die Selbstzeugnisse wie beispielsweise die nur rudimentär erhaltenen Notizen und Korrespondenzen der von 1832 bis 1852 in Göttingen wirkenden Botaniker*innen sowie Dokumentationen zur Lehrpraxis. Ebenso muss an dieser Stelle deutlich gemacht werden, dass ein Herbarium eigenen Ordnungsprinzipien folgt, die kulturwissenschaftlichen Fragestellungen nicht immer in die Hände spielen. Denn in einem Herbarium wird in erster Linie nach wissenschaftlichen Pflanzennamen gesucht. ${ }^{37}$ Die Sammlung verfügt zwar über eine eigene Datenbank, doch sind dort bisher vor allem die ungefähr 14.000 Belege der Typensammlung sowie die historisch bedeutsamen Herbarbelege eingespeist worden. Eine umfängliche Digitalisierung und Verzeichnung der Sammlung steht noch aus. Das hat zur Folge, dass eine Recherche nach Sammler*innen, nach Daten und Sammlungsorten stets nur in Bezug auf einen Ausschnitt der Sammlung vorgenommen werden kann. Da der digitalisierte Teil des Herbariums aber immerhin aus etwa 35.000 Herbarbelegen besteht, ermöglichte diese Begrenzung auf der anderen Seite auch überhaupt einen ersten Zugang zu der 800.000 Exemplare umfassenden Sammlung. ${ }^{38}$

Diesem vielschichtigen Korpus widmet sich die Arbeit aus einer praxeologischen und gleichzeitig dinganalytischen Perspektive. Damit verfolgt sie das theoretische sowie methodische Ziel, besonders den Sammlungsobjekten in einem

35 Die Rechnungsunterlagen des Göttinger Herbariums waren besonders für die Rekonstruktion der Sammlungsbestände hilfreich und stellten vielfach eine wichtige Ergänzung zum 1878 nachträglich angefertigten Sammlungskatalog dar.

${ }^{36}$ Bestandteil der Personalakten waren zum Beispiel auch die in Vorbereitung von Forschungsexkursionen verfassten Schreiben an Kuratorium und Sekretariat sowie die im Anschluss einzureichenden Reiseberichte.

${ }^{37}$ Eine Ausnahme stellen die historischen Bestände sowie das Herrenhäuser Herbarium dar, die als geschlossene Konvolute behandelt und zum Teil in gesonderten Räumlichkeiten des Instituts abseits der Haupt- und Typensammlung aufbewahrt werden.

38 Vgl. Herbarium Göttingen. 
erweiterten Verständnis zu begegnen: Sie werden als Glied und Teilhabende innerhalb der Wissenserzeugung und Wissensvermittlung betrachtet, die aus diesem Grund weder allein durch ihre materielle Beschaffenheit zu erschließen noch einfach zu dekodieren sind. Vielmehr werden die Sammlungsobjekte immer im Wechselspiel sozialer Praktiken, als eingebunden in komplexe Ketten und Netze von Repräsentationen wahrgenommen: „[...] the history of science is not a history of concepts, or at least not that alone, but a history of the making and using of objects to understand the world." 39 Der praxisbezogene Ansatz ermöglicht es dabei, eine Perspektive auf die Erzeugung von Wissen zu ermöglichen, die neben den involvierten Personen und ihren Handlungsweisen auch die Dinge und Räume als Akteure und Ko-Akteure von Wissen(schaft) versteht. ${ }^{40} \mathrm{Um}$ diese methodische Vorgehensweise auch formal abzubilden, gliedert sich der Hauptteil der Arbeit in fünf Kapitel, von denen jedes eine Praxis näher beleuchtet. Der Materialität des Wissens Rechnung tragend, werden diese wiederum von jeweils zwei Objektbeschreibungen eingeleitet. Sie dienen an dieser Stelle aber nicht als Illustration der Ergebnisse, sondern sollen vielmehr das Wechselverhältnis von wissenschaftlicher Praxis und Objekterstellung verdeutlichen. Ein solches Vorgehen möchte darüber hinaus genau jenes Wechselspiel von Nähe und Distanz schaffen, mit dem sowohl Details fokussiert als auch das Beobachtete und Beschriebene wieder in einen breiteren Kontext gesetzt werden können und das für kulturanthropologische Perspektivierungen ausschlaggebend ist.

${ }^{39}$ Cook et al. 2017, S. 12.

${ }^{40}$ Vgl. Mariss 2015, S. 92. 

2 Eine historische Ethnografie und Praxeografie 


\subsection{Die Sammlung öffnen}

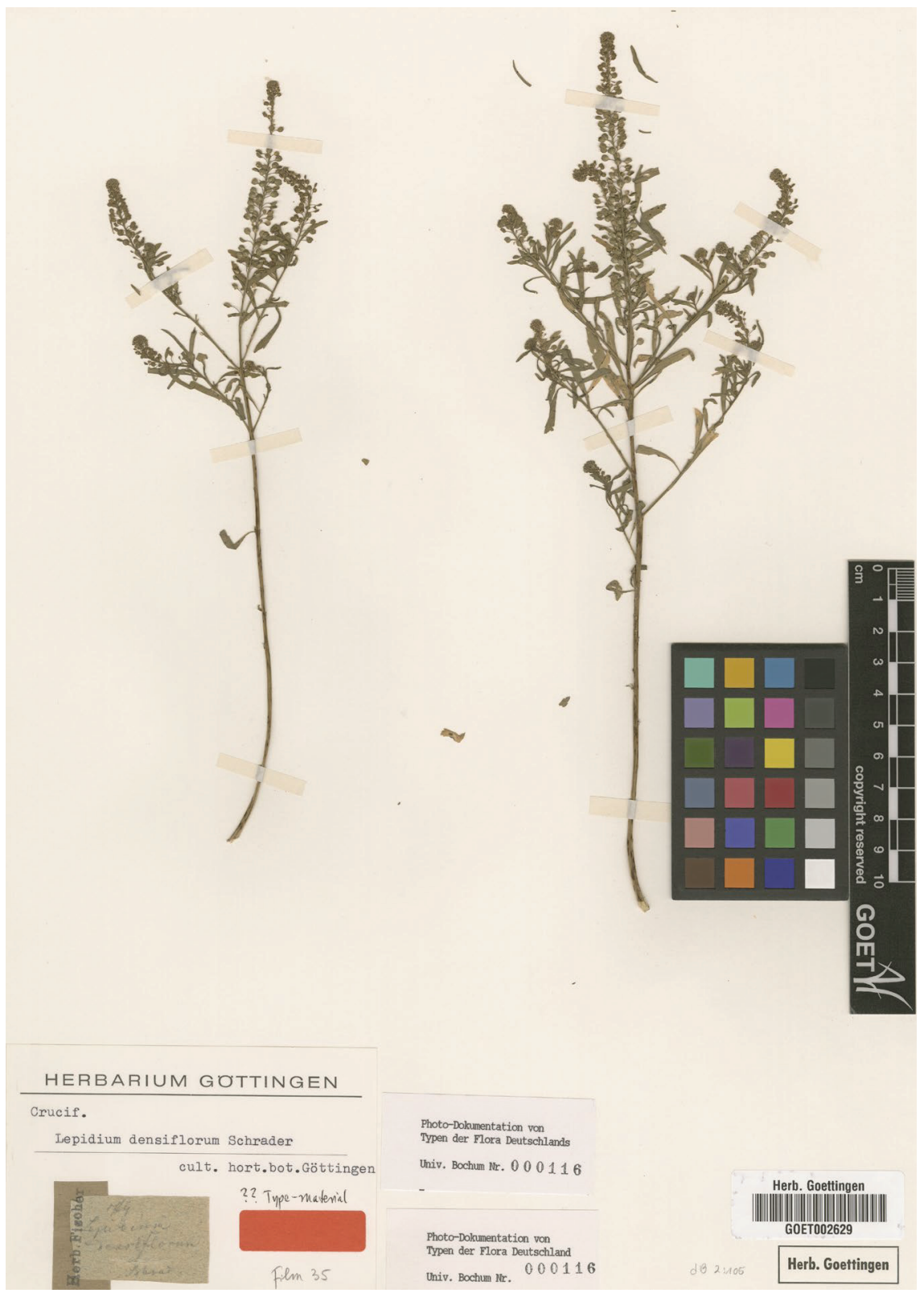

Abb. 1: Herbarbeleg von Lepidium densiflorum Schrad., kultiviert im botanischen Garten Göttingen, Herbarium Göttingen, GOET002629. 
Zwei Sprosse der Pflanze Lepidium densiflorum Schrad. ${ }^{41}$ wurden mit mehreren selbstklebenden Papierstreifen nebeneinander auf einem 28,5 x $41 \mathrm{~cm}$ großen weißen Papierbogen fixiert. Dabei ist das linke Exemplar etwas kürzer als das rechte. Zwar haben sich einige Pflanzenteile gelöst, doch ist der Beleg insgesamt in einem sehr guten Zustand erhalten.

Während der Großteil des Papierbogens vom Präparat selbst eingenommen wird, beherrscht den unteren Teil des Pflanzenbelegs eine überdurchschnittlich hohe Anzahl zusätzlicher Informationen in Form von Etiketten sowie Notizen und Stempeln. Das größte der Etiketten trägt in Großbuchstaben die Überschrift ,Herbarium Göttingen‘ und gibt darunter die Pflanzenfamilie (Cruciferae) sowie den wissenschaftlichen Namen der präparierten Pflanze an. Der Zusatz ,cult. hort. bot. Göttingen' macht außerdem kenntlich, dass diese im botanischen Garten der Universität kultiviert worden ist. In der rechten unteren Ecke wurden untereinander der Barcode mit Inventarnummer sowie der Stempel des Herbariums platziert. Während diese drei Informationen auf allen Herbarbögen zu finden sind, handelt es sich bei den übrigen Angaben um spezielle Zusätze. So erhalten nur diejenigen Belege einen roten Aufkleber, bei denen es sich um einen Pflanzentypus, also das Exemplar, an dem eine Art erstmals beschrieben wurde, handelt. Dass dies bei der vorliegenden Pflanze allerdings nicht ohne Zweifel behauptet werden kann, verrät die mit einem Kugelschreiber gemachte Notiz ,?? Type-material'. Weiter deuten zwei mit einer Schreibmaschine erstellte Etiketten darauf hin, dass dieser Beleg für eine Fotodokumentation der Universität Bochum über die Typen der Flora Deutschlands genutzt wurde. Die Bleistiftnotizen verweisen zum einen auf die Negativsammlung eines ehemaligen Göttinger Botanikers und zum anderen auf eine früher genutzte Datenbank.

Eigens für den Scan wurden dem Papierbogen außerdem am rechten Rand ein Farbkeil sowie ein Maßband beigelegt. Sie erlauben dem*der Betrachter*in bereits anhand des Digitalisats eine Einschätzung zu Farbigkeit und Größe des Pflanzenbelegs vorzunehmen. Beide werden jedoch direkt nach dem Scanvorgang wieder entfernt, sodass sie nicht eigentliche Bestandteile des Herbarbelegs darstellen.

Lässt man das Präparat selbst außer Acht, handelt es sich bei den bisher beschriebenen Bestandteilen des Pflanzenbelegs um Informationen jüngeren Datums. Einzig die zwei Etiketten aus braunem Papier in der linken unteren Ecke verweisen auf den Originalbeleg. Das Papierstück mit dem Stempelaufdruck ,Herb. Fischer stammt von dem ursprünglichen Papierbogen, der später durch das neue Exemplar aus anderem Material ersetzt worden ist. Es gibt an, dass der

\footnotetext{
${ }^{41}$ Wissenschaftliche Pflanzennamen bestehen stets aus zwei Teilen, dem Gattungs- und dem Artnamen, die in ihrer Kombination einzig sind. Der daran anschließende Personenname verweist darüber hinaus auf den*die Erstbeschreiber*in der Art. Da, bis auf Lepidium densiflorum Schrad., alle aus dem Quellenmaterial stammenden und in dieser Arbeit angeführten Pflanzenbezeichnungen heute keine Gültigkeit mehr besitzen, wird bei jeder Erstnennung der aktuell akzeptierte Pflanzenname in Klammern mit angegeben.
} 
Beleg aus der Pflanzensammlung des früheren Garteninspektors Christian Abraham Fischer (1785-1836) stammt. Bei dem zweiten Zettel handelt es sich um einen Teil des Originaletiketts, das höchstwahrscheinlich von Heinrich Adolf Schrader (1767-1836), dem Beschreiber der Pflanze, selbst angefertigt worden ist. Es gibt genauso wie das heutige Etikett den wissenschaftlichen Namen der präparierten Pflanze an. Darüber hinaus verweist es darauf, dass der Beleg von Schraders Sammlung in diejenige Fischers gelangt ist. 


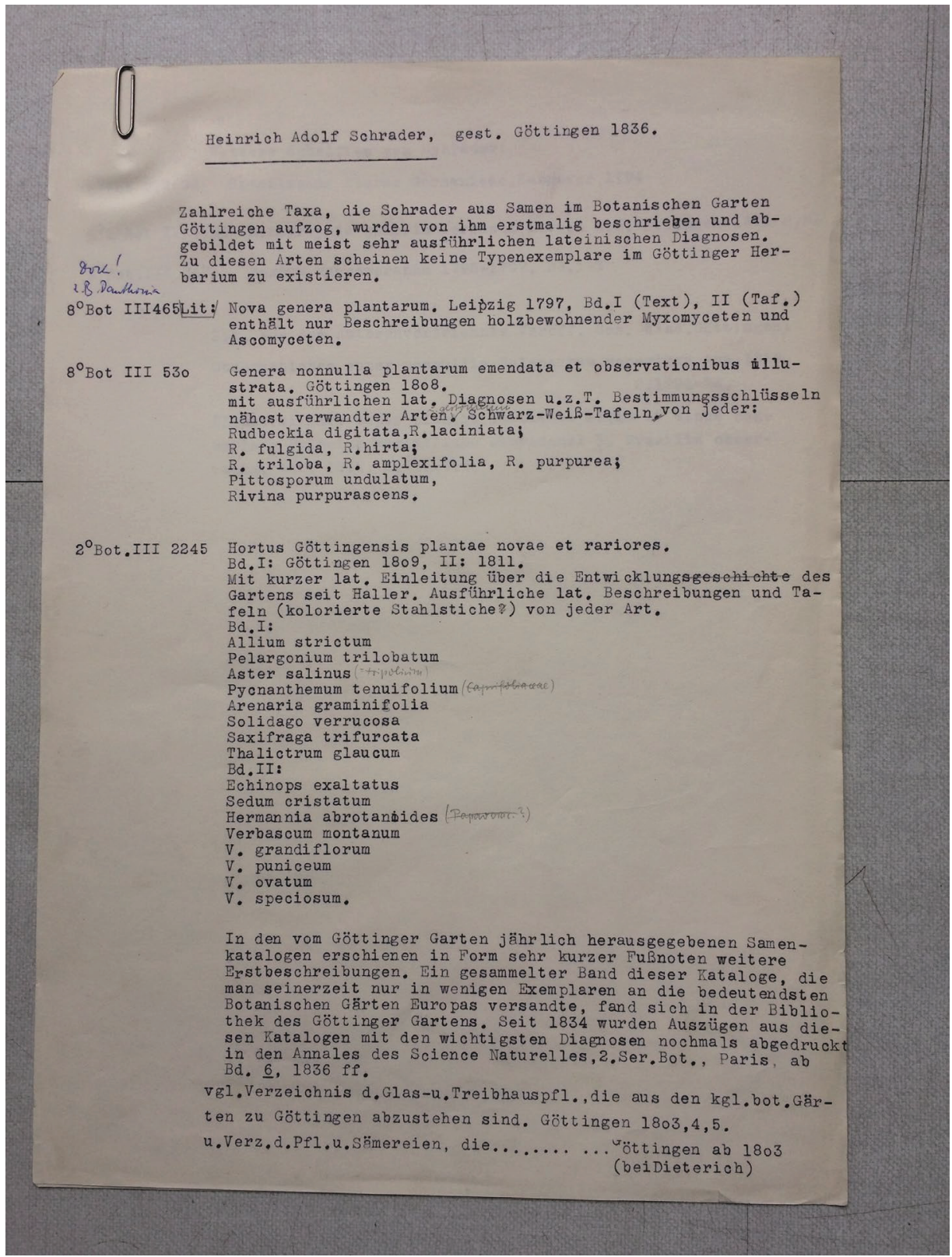

Abb. 2: Verzeichnis von Veröffentlichungen von H. A. Schrader (mit Standnummern in U.B.), in denen neue Arten beschrieben wurden, mit Aufzäblung einiger dieser Arten, Seite 1, Albrecbt-von-HallerInstitut für Pflanzenwissenschaften, PWI.Syst.Geobotan.HE15. 


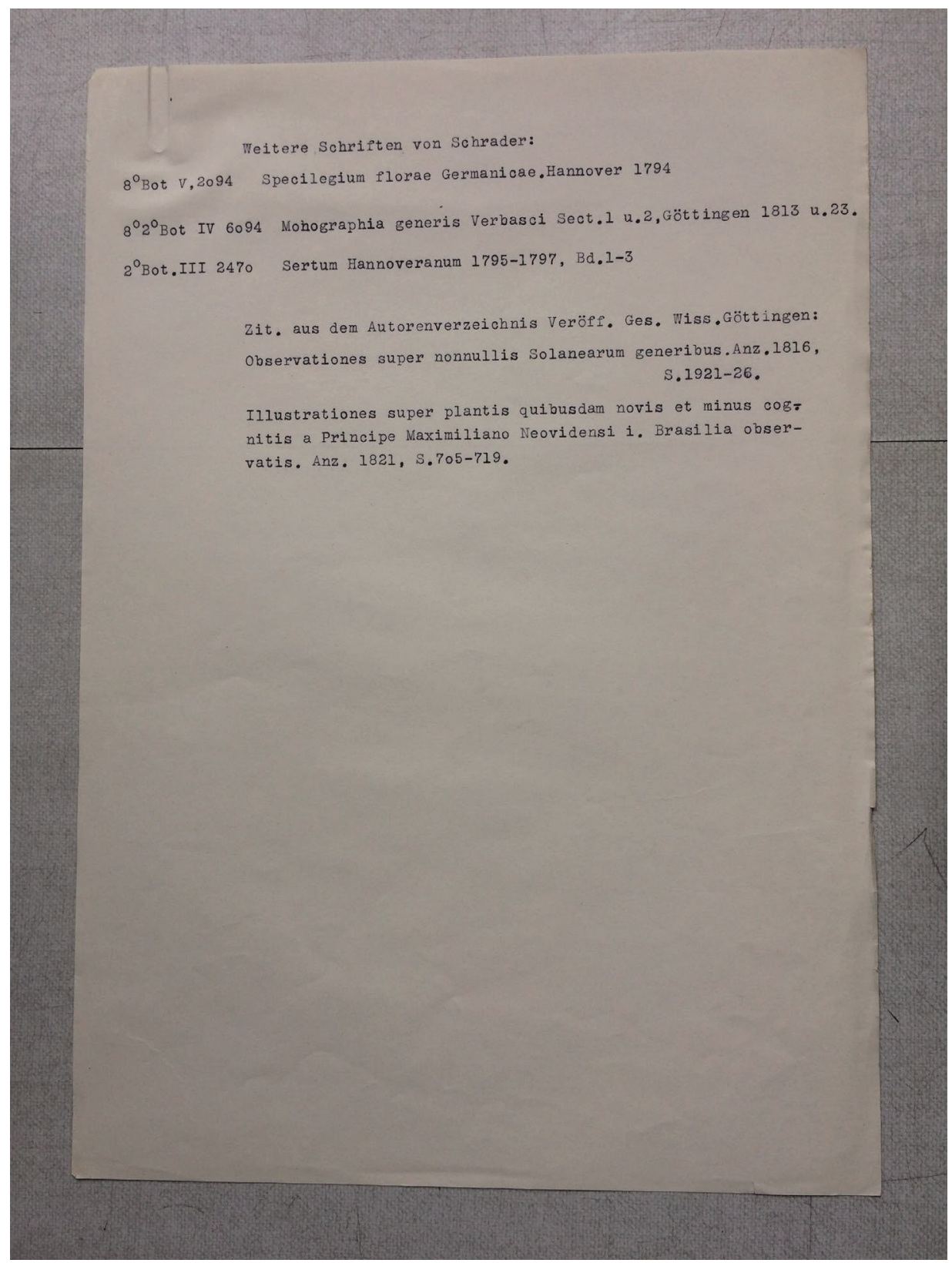

Abb. 3: Verzeichnis von Veröffentlichungen von H. A. Schrader (mit Standnummern in U.B.), in denen neue Arten beschrieben wurden, mit Aufzählung einiger dieser Arten, Seite 2, Albrecht-von-HallerInstitut für Pflanzenwissenschaften, PWI.Syst.Geobotan.HE15. 
Unter der Signatur HE15 im Archiv des Herbariums eingeordnet und in einer Mappe aufbewahrt, befinden sich zwei durch eine metallene Büroklammer zusammengehaltene und auf der Vorderseite mit einer Schreibmaschine bedruckte DIN A4-Seiten. Unter der Überschrift ,Heinrich Adolf Schrader, gest. Göttingen 1836.' werden nach einem einführenden Absatz jene Veröffentlichungen dieses Göttinger Botanikers angeführt, in denen er neue Arten beschrieb. Insgesamt sechs Werke werden gemeinsam mit einer Bibliothekssignatur und einer kurzen Information zum Inhalt aufgelistet. Auch werden teilweise Hinweise zu enthaltenen Abbildungen gegeben und einige der beschriebenen Arten benannt. An manchen Stellen wurde mit Bleistift korrigiert oder ergänzt. Wann und von wem diese Liste erstellt wurde, ist nicht bekannt. Unklar ist auch, ob sie Vollständigkeit für sich beanspruchen kann und nach welchem Kriterium die Publikationen aufgeführt wurden.

Die Aufmerksamkeit soll an dieser Stelle aber auf den ersten Absatz gelenkt werden: ,Zahlreiche Taxa, die Schrader aus Samen im Botanischen Garten Göttingen aufzog, wurden von ihm erstmalig beschrieben und abgebildet mit meist sehr ausführlichen lateinischen Diagnosen. Zu diesen Arten scheinen keine Typenexemplare im Göttinger Herbarium zu existieren.' Denn an dieser Stelle griff ein zusätzlicher Akteur ein und notierte mit einem Kugelschreiber widersprechend: ,Doch! z. B. Danthonia'.

Listen, die einen Überblick über botanische Werke von Wissenschaftler*innen zu geben suchen, sind nicht unüblich und liegen im Archivbestand des Göttinger Herbariums mehrfach vor. Doch in Zusammenhang mit dem Herbarbeleg der Pflanze Lepidium densiflorum wird offenbar, dass in der Unstimmigkeit zwischen Autor*in der Auflistung und Korrektor*in ein Irritationsmoment begründet liegt, das den*die Listenverfasser*in überhaupt erst zu der Aufnahme seiner kurzen Anmerkung in das Dokument veranlasste:

Der Botaniker Heinrich Adolf Schrader war von 1797 bis zu seinem Tod in Göttingen tätig und seit 1803 auch Direktor des botanischen Gartens. ${ }^{42}$ Wie die beschriebene Liste zeigt, brachte er in der Zeit von 1794 bis 1823 verschiedene Werke zur Veröffentlichung, die auf Grundlage von Pflanzenexemplaren und ihrer Beschreibung entstanden. Um den Verbleib genau dieser Pflanzenbelege entstand die Uneinigkeit zwischen dem*der Autor*in der Liste und dem*der Urheber*in der kleinen Seitennotiz. Offenbar war der*die Verfasser*in irritiert davon, dass keine oder - in Berücksichtigung der korrigierenden Notiz - zumindest nur ein geringer Teil der beschriebenen Pflanzen im Göttinger Herbarium aufzufinden waren. Zumindest erschien ihm dieser Hinweis für die Vollständigkeit der Liste nicht verzichtbar. Auch wenn die genaue Anzahl der aus Schraders

42 Vgl. Wagenitz 1982, S. 150; Wagenitz 1988, S. 158. 
Herbarium stammenden und im Göttinger Herbarium aufbewahrten Exemplare nicht exakt zu bestimmen ist, kann konstatiert werden, dass sie im Vergleich zu anderen Göttinger Botaniker*innen deutlich geringer ist. ${ }^{43}$ Die Etiketten des beschriebenen Pflanzenbelegs legen die Vermutung nahe, dass dieser aus Schraders Herbarium selbst stammt. ${ }^{44}$ Gleichzeitig lassen sie aber auch erkennen, dass er nur für eine begrenzte Zeit Teil desselben gewesen war und zuletzt im Herbarium des Inspektors des botanischen Gartens Göttingen und im gleichen Jahr wie Schrader verstorbenen Fischer aufbewahrt worden ist. ${ }^{45}$ Als Bestandteil dessen Sammlung, die am 15. April 1837 angekauft wurde, gelangte der Beleg in das Göttinger Herbarium. ${ }^{46}$ Die eigentliche Sammlung Schrader wurde nicht erworben. ${ }^{47}$ Zwar stand das Kuratorium auch mit der Witwe Schrader in Verhandlung und wurde der Wert des Herbariums auf sein Ansuchen hin durch Friedrich Gottlieb Bartling (1798-1875) ausführlich dargelegt und als beachtlich eingestuft, doch ist davon auszugehen, dass der geforderte Kaufpreis als zu hoch erachtet wurde. ${ }^{48}$ Auch Schraders Korrespondenz wurde von seiner Frau nicht an das

Kuratorium abgetreten, da sie die möglicherweise darin enthaltenen Ideen für Publikationen als Privatsache ihres verstorbenen Mannes betrachtete. ${ }^{49}$ Lediglich

${ }^{43} \mathrm{Da}$ der Bestand des Herbariums bisher nicht vollständig digitalisiert und erfasst worden ist, können in dieser Hinsicht keine gesicherten Aussagen getroffen werden.

${ }^{44}$ Eine Zuordnung von Herbarbelegen zu bestimmten Sammler*innen und Forschenden ist häufig über einen Schriftabgleich möglich. Eine Auswahl der dafür notwendigen Schriftproben stellt der von Wagenitz für das Göttinger Herbarium verfasste Index collectorum principalium bereit. Vgl. Wagenitz 1982, S. 198-214.

${ }^{45}$ Vgl. ebd., S. 54.

46 Die Sammlung Fischer wurde anstelle der Sammlung Schrader für das Herbarium angekauft. Sie galt jedoch als weniger wertvoll, da es sich bei den enthaltenen Exemplaren vor allem um Pflanzen aus dem botanischen Garten handelte und sie nur einzelne wenige Belege anderer Sammler*innen mit einschloss. Aus der Einschätzung Bartlings geht vielmehr hervor, dass das Fischer-Herbarium als Ersatz der Schraderschen Pflanzensammlung angesehen wurde, da auch diese einige Pflanzen des botanischen Gartens enthielt. Vgl. UniA GÖ, Kur., 5586, Schreiben Bartlings an das Universitätskuratorium, 17.2.1837.

${ }^{47}$ Bartling wird in einem Schreiben vom 14. März 1837 über den Beschluss des Kuratoriums informiert. Vgl. UniA GÖ, Kur., 5586, Schreiben des Universitätskuratoriums an Bartling, 14.3.1837.

48 Vgl. Wagenitz 1982, S. 6.

49 Vgl. UniA GÖ, Sek., 450.4, Schreiben des Prorektors und des Senats an das Universitätskuratorium, 11.12.1836. Tatsächlich erschienen die nicht veröffentlichten wissenschaftlichen Arbeiten aus Schraders Nachlass dann 1838 in Verbindung mit seinem Nachruf. Vgl. o. A. 1838; Wunschmann 1971a, S. 430. Am Beispiel der Verhandlungen über den Nachlass Schraders zeigt sich exemplarisch, welche zentrale Rolle die Witwe des Botanikers hierbei spielte. Sie wurde nach dem Tod ihres Mannes zur Ansprechpartnerin für die Universität und entschied über den Verbleib der Sammlung. Es erscheint deshalb auch in Hinblick auf das Göttinger Herbarium sinnvoll, der sozialen Funktion der Professorenfrauen verstärkte Aufmerksamkeit zu widmen. Vgl. Mariss 2018, S. 218-222; Panke-Kochinke 1993. 
Schraders Dienstpapiere sowie einige botanische Zeichnungen, die dieser durch Mittel des Kuratoriums erstellen ließ, mussten an dieses abgetreten werden. ${ }^{50}$

Die Verwunderung des*der Listenverfassers*in über die vergleichsweise geringe Anzahl von Typenexemplaren der von Schrader beschriebenen Arten in der Göttinger Sammlung gründet demnach in der Annahme, dass die Sammlungen Göttinger Botaniker*innen auch in Form einer repräsentativen Menge entsprechender Exemplare vor Ort anzufinden sein müssten. An dieser Stelle treffen unterschiedliche Sammlungspraktiken aufeinander, deren Auswirkung auf den Sammlungsbestand besonders mit Blick auf die Gründungsphase des Göttinger Herbariums sichtbar wird. Die mit der Gründung getroffenen Regelungen und Verordnungen nahmen direkten Einfluss auf die Sammlungspraxis. Das sich bei dem*der Autor*in der Liste herauskristallisierende Selbstverständnis bezüglich einer Sammlungspraxis - nämlich das Öffentlich- und Zugänglichmachen sowie das Bereitstellen der Forschung vor Ort - wird, so die These, mit der Gründung des Herbariums am 26. Oktober 1832 institutionalisiert. ${ }^{51}$

Besonders die der Gründung vorausgehenden Briefwechsel und die darin enthaltenen Positionierungen bilden ab, wie unterschiedliche Sammlungspraktiken gegeneinander abgewogen und verhandelt wurden. In dem fast zwei Jahre andauernden Aushandlungsprozess wurde nicht nur für, sondern auch gegen die Einrichtung eines Herbariums argumentiert und Meinungen sowie Einschätzungen verschiedener Akteure eingeholt. Aus diesen lassen sich deutlich die mit der Gründung einhergehenden Interessen und Hoffnungen, aber auch Befürchtungen herausstellen. Antreibende Kraft in diesem Prozess war der Botaniker und zu dieser Zeit noch Privatdozent Bartling, der sich für „die Anlegung eines öffentlichen Herbariums“52 aussprach. Gegenargumente brachte in erster Linie der dienstältere Professor und Direktor des botanischen Gartens Heinrich Adolf Schrader hervor. ${ }^{53}$

${ }^{50}$ Vgl. UniA GÖ, Sek., 450.4, Schreiben des Prorektors und des Senats an das Universitätskuratorium, 11.12.1836; UniA GÖ, Sek., 450.4, Schreiben des Universitätsgerichts an die Hofrätin Schrader, 18.3.1837.

51 Vgl. UniA GÖ, Kur., 5584, Schreiben des Universitätskuratoriums an Bartling, 26.10.1832.

52 UniA GÖ, Kur., 5584, Schreiben Bartlings an das Universitätskuratorium, 8.12.1830.

${ }^{53}$ Die Gegenargumentation zu Bartlings Vorschlag wurde dem Kuratorium von Johann Friedrich Blumenbach (1752-1840) übermittelt. Dieser war gebeten worden, dem Kuratorium eine Einschätzung zu geben und hatte sich offensichtlich zu diesem Zweck mit Schrader verständigt. Vgl. UniA GÖ, Kur., 5584, Schreiben des Universitätskuratoriums an den Obermedizinalrat Blumenbach, 15.3.1832; UniA GÖ, Kur., 5584, Schreiben des Obermedizinalrats Blumenbach an das Universitätskuratorium, 2.4.1832. Das Verhältnis zwischen Schrader und Bartling war offenbar schon zuvor angespannt gewesen. So ermöglichte Schrader dem Privatdozenten Bartling nur eine eingeschränkte Nutzung des botanischen Gartens. Vgl. UniA GÖ, Kur., 5579. 
Bartling, der sich am 8. Dezember 1830 erstmalig mit einem Pro memoria an das Kuratorium in Hannover wandte, wünschte eine öffentliche Sammlung, die vor allem auch für Studierende zugänglich sein sollte. ${ }^{54}$ Denn in Vorbereitung auf den im Sommer 1793 stattgefundenen Umzug des Academischen Museums als Folge der Ausbaumaßnahmen der Universitätsbibliothek wurden alle botanischen Sammlungen 1792 an den damaligen Direktor des botanischen Gartens Prof. Georg Franz Hoffmann (1760-1826) abgegeben. ${ }^{55}$ Seitdem befanden sie sich in einem Zimmer der sich auf dem Gelände des botanischen Gartens befindlichen Offizialwohnung des Direktors, der diese auch verwaltete. ${ }^{56}$ Darüber hinaus hatte Schrader, wie er selbst in seinem Schreiben betonte, die Sammlungen durch ein Garten-Herbarium ergänzt sowie durch Ankäufe aus dem Ausland durch private Mittel erweitert. ${ }^{57}$ Eine Einsicht in die Sammlung wurde aber lediglich auf Anfrage hin genehmigt. ${ }^{58}$ In seiner Gegenargumentation zu Bartlings Vorschlag bezüglich der Einrichtung eines öffentlichen Herbariums schrieb er:

„Alle Sammlungen der Art, wenn sie auch auf das Beste angefertigt sind, können von den Gegenständen - den Pflanzen, die sie enthalten - nur ein schwaches Bild geben, folglich dem Anfänger der Botanik von gar keinem Nutzen seyn. Diese belehren sich besser in der Natur, und, wo ein nur mittelmässig eingerichteter Garten ist, noch besser durch einen solchen, da er alle Hülfsmittel in dieser Hinsicht darbietet. Mehr Nutzen gewähren schon die Herbarien dem Kenner, nicht so wohl zur Rückerinnerung des früher Gesehenen, als auch besonders zur Vergleichung mit andern, ihm etwa vorkommenden zweifelhaften Gewächsen. Jeder Botaniker ist daher auch gegenwärtig mit einer so grossen Sammlung versehn, als es für seine Bedürfnisse erforderlich ist.“59

Weiter führte er aus:

„Studenten und überhaupt Anfänger der Botanik wissen den Werth solcher Sammlungen nicht zu schätzen, schaden denselben wenn auch nur aus Unkunde, und müssen daher möglichst entfernt gehalten werden. Männern aber von Fach gestattet man gern den Zutritt, wenn sie sich gehörigen Orts melden, oder man gibt ihnen auch wohl, gegen einen ausgestellten Schein, auf kurze Zeit die gewünschten Pflanzen zur genauern Vergleichung.“60

${ }^{54}$ Vgl. UniA GÖ, Kur., 5584, Schreiben Bartlings an das Universitätskuratorium, 8.12.1830.

55 Vgl. Nawa 2010b, S. 34; Plesker 2015, S. 417; UniA GÖ Sek., 450.4, Liste über die Sammlungen und Herbarii vivi, die das Academische Museum an Hoffmann abgegeben hat, 6.6.1792.

Die Auflistung wurde zu einem späteren Zeitpunkt von Schrader ergänzt. Vgl. UniA GÖ, Sek., 450.4, Nachtrag zur Liste von Prof. Hoffmann, verfasst von Schrader, 2.11.1808. Die Beweggründe für die Abgabe der Bestände erläutert Anne Mariss. Vgl. Mariss 2018, S. 216. Zur Geschichte des Academischen Museums vgl. Nawa 2010a.

56 Vgl. UniA GÖ, Kur., 5584, Schreiben Schraders an den Obermedizinalrat Blumenbach, 31.3.1832.

57 Vgl. UniA GÖ, Kur., 5584, Schreiben Schraders an den Obermedizinalrat Blumenbach, 31.3.1832.

${ }^{58}$ Vgl. ebd.

${ }^{59}$ Ebd.

${ }^{60}$ Ebd. 
Deutlich wird, dass Schrader zum einen explizit zwischen Studierenden (als den Anfängern) und den Kennern der Botanik unterschied und zum anderen auch die beiden Institutionen, botanischer Garten und Herbarium, als hierarchisch in ihrer Funktion und ihrem Nutzen verstand. Anders als Bartling sprach er es den Studierenden vollkommen ab, sinnvoll von den Sammlungen getrockneter Pflanzen Gebrauch machen zu können. Die Arbeit mit diesen war aus seiner Perspektive vielmehr den eigentlichen Fachvertreter*innen vorbehalten. Und da Botaniker*innen selbst über (Privat-)Sammlungen verfügten, erschien ihm die Einrichtung eines öffentlichen Herbariums schlichtweg überflüssig. Diese Position unterstrich er zusätzlich durch eine genaue Auflistung der in Göttingen vorhandenen Pflanzensammlungen. Schließlich verfügte Göttingen, so Schrader, ja bereits über einen nicht unansehnlichen Sammlungsbestand. Mit dem Wunsch, „durch ähnliche Gesuche u. Vorschläge des Herrn Prof. Bartling nicht weiter gestört [zu] werden" "61 beendete er seine Stellungnahme.

Doch gerade mit der Aussage, Göttingen besäße bereits eine umfassende Pflanzensammlung, spielte Schrader Bartlings Argumentation direkt in die Hände. Denn in Bartlings Reaktion auf Schraders Schreiben betonte dieser, bis dato keine genaue Kenntnis über die benannten Bestände gehabt zu haben. Er selbst kannte „nur einige auf dem Museum aufbewahrte zum Theil unbrauchbar gewordene Fascikel sibirischer Pflanzen"62, also denjenigen Teil der Sammlung, der auch öffentlich zugänglich gewesen war. Damit war laut Bartling offenkundig, dass das Vorhandensein der Sammlungen nur wenigen bekannt sein konnte und diese, da ihre Benutzung ,nur durch eine besondere Begünstigung des Hofraths Schrader möglich wäre, bisher nicht unmittelbar das Studium der Botanik in Göttingen gefördert hatten “63.

Darüber hinaus differenzierte er im Gegensatz zu Schrader gegenüber dem Kuratorium ganz explizit zwischen privater und universitärer Sammlung. Er stellte heraus, dass Schrader in seinem Verzeichnis seine eigene Sammlung mit den Sammlungen der Universität vermengt habe, diese aber getrennt voneinander zu betrachten seien. ${ }^{64}$ Wenn es um die Frage ginge, ob die bereits vorhandenen botanischen Sammlungen den Bedürfnissen der Forschung und Lehre genügten, so seien alle Exemplare, die aus eigenen Mitteln Schraders angeschafft wurden, nicht mit zu berücksichtigen. ${ }^{65}$

\footnotetext{
${ }^{61}$ Ebd.

${ }^{62}$ UniA GÖ, Kur., 5584, Schreiben Bartlings an das Universitätskuratorium, 9.9.1832.

${ }^{63}$ Ebd.

${ }^{64} \mathrm{Vgl}$. ebd.

${ }^{65}$ Vgl. ebd.
} 
Genau wie Schrader vertrat auch Bartling die Position, dass eine Beobachtung am Objekt stets vorzuziehen sei. ${ }^{66}$

\begin{abstract}
„[D]enn so unentbehrlich auch eine gewählte Bibliothek für den Botaniker ist, so muss doch jede allein aus Büchern geschöpfte Kenntnis der Natur mangelhaft und unsicher bleiben und kann nie den Werth einer auf eigener Anschauung begründeten erreichen. “67
\end{abstract}

Dabei reicht laut Bartling ein botanischer Garten allein allerdings nicht mehr aus. „Nur die glückliche Vereinigung aller Hülfsmittel [...]"68 könne den Herausforderungen heutiger Forschung gerecht werden. Die Bedeutung eines Herbariums für die botanische Forschung schätzt Bartling anders als Schrader ebenso groß ein, wie die eines botanischen Gartens - und das für Gelehrte und Studierende gleichermaßen. ${ }^{69}$

,Die Sammlung öffnen' bedeutet mit Blick auf das Göttinger Herbarium die Entwicklung von einem Konglomerat privater Sammlungen hin zu der Institutionalisierung und dem Entstehen einer systematisierten öffentlichen Sammlung, die auf Nutzung und Zugänglichkeit ausgerichtet ist. Wenn also dem Göttinger Herbarium die Sammlungen der in der Zeit von 1832 bis 1852 tätigen Botaniker*innen in unterschiedlicher Dichte erhalten sind, so ist dies nicht allein Ausdruck des persönlichen Bestrebens oder Interesses derjenigen Wissenschaftler*innen. Vielmehr ist diese Beobachtung über den Sammlungsbestand auf eine Veränderung in der Sammlungspraxis zurückzuführen. Die Entwicklung von der privaten hin zur öffentlichen Sammlung hat nicht nur zur Gründung des Herbariums geführt, sondern sich gleichzeitig auch in den Sammlungsbestand selbst eingeschrieben.

So ließe sich das Vorhandensein zahlreicher Herbarbelege des Göttinger Botanikers Bartling und die Abgabe seines Herbariums an die Universität leicht als ausschließlicher Akt der Verbundenheit mit der durch ihn gegründeten Sammlung interpretieren. Der Verkauf seiner Sammlung am 23. August 1841 muss aber auch vor dem Hintergrund der am 18. Januar 1838 in Kraft getretenen Dienstinstruktion betrachtet werden. ${ }^{70}$ Denn dieses Reglement untersagte dem Direktor erstmalig den Besitz einer eigenen Sammlung. Gleichzeitig schrieb es ihm vor, sein bis zum Antritt der Direktorenstelle zusammengetragenes Herbarium dem Kuratorium in Hannover zum Verkauf anzubieten. Dabei darf selbstverständlich nicht außer Acht gelassen werden, dass diese Instruktionen von Bartling selbst aufgestellt und dem Kuratorium vorgelegt worden sind. Neben einer sicherlich von ihm gewünschten finanziellen Absicherung verfolgte er damit aber ein

\footnotetext{
${ }^{66}$ Vgl. UniA GÖ, Kur., 5584, Schreiben Bartlings an das Universitätskuratorium, 8.12.1830.

Zur Beobachtung als Methode vgl. Daston 2011.

${ }^{67}$ UniA GÖ, Kur., 5584, Schreiben Bartlings an das Universitätskuratorium, 8.12.1830.

${ }^{68}$ Ebd.

${ }^{69}$ Vgl. ebd.; UniA GÖ, Kur., 5584, Schreiben Bartlings an das Universitätskuratorium, 22.10.1831.

${ }^{70}$ Vgl. PWI.Syst.Geobotan.A1.
} 
wissenschaftliches Interesse und es war offenkundig sein Anliegen, die Sammlungspraxis des Göttinger Herbariums auf lange Sicht zu verändern. Denn nicht erst mit dem Inkrafttreten der Instruktionen 1838, sondern bereits ab 1832 sammelte Bartling für das Herbarium und nahm alles Akquirierte in die Sammlung der Universität auf. ${ }^{71}$

Das Abfassen der Vorschriften ist deshalb vielmehr als eine rechtliche Absicherung einer von ihm bereits verfolgten Praxis zu verstehen. Und diese Sammlungspraxis fasste allmählich Fuß: Die Sammlung August Heinrich Rudolf Grisebachs (1814-1879), die heute zum wertvollsten Bestandteil des Göttinger Herbariums gehört, wurde nicht erst 1875 mit der in Folge des Ablebens Bartlings erfolgten Übernahme der Direktorenstelle abgegeben. Grisebach vermachte sein Privatherbar bereits 1866 der Universität als Legat und wurde im Gegenzug in dessen Ausbau mit einer jährlichen Zahlung von 100 Talern finanziell unterstützt. ${ }^{72}$ Die Abgabe mag durchaus wie auch bei Bartling aus ideellen Gründen und dem Wunsch nach finanziellem Ausgleich geschehen sein. Als Erklärung reicht dies allein aber nicht aus, sondern es muss auch der Verbleib der Sammlung Grisebach im Rahmen veränderter Sammlungspraktiken betrachtet werden. Durch rechtliche Regelungen sowie durch die finanzielle Beteiligung des Kuratoriums wurden die Sammlungen Bartlings und Grisebachs schrittweise an den Standort Göttingen gebunden. Eine langwierige Verhandlung, wie sie noch im Fall des Nachlasses Schraders geführt worden war, ist nicht mehr nötig gewesen. Universitäts- und Gartenherbarium sollten mit der Gründung eines öffentlichen Herbariums und mit dem Inkrafttreten der neuen Dienstinstruktion ,vollen Ersatz für die im Privatbesitz des Garten-Directors befindlich gewesenen Sammlungen, sowohl hinsichtlich des botanischen Gartens, als der botanischen Studien überhaupt gewähren"73.

Damit fügt sich die Institutionalisierung des Göttinger Herbariums in einen weitaus breiteren Entwicklungsrahmen ein. Die Tatsache, dass Privatsammlungen nicht auf Dauerhaftigkeit angelegt waren und der Wissenschaft häufig nur für eingeschränkte Zeit und auch nur einem eng begrenzten Personenkreis zur Verfügung standen, führte nicht nur in Göttingen zu ihrer Eingliederung in wissenschaftliche Institutionen. ${ }^{74}$ Dieser Aushandlungsprozess zwischen privater und öffentlicher Sammlung wurde bereits im 18. Jahrhundert angestoßen. ${ }^{75}$

Während die Bestrebungen um Zugänglichkeit und Nutzbarmachung von Sammlungen in Bezug auf das Museumswesen bereits intensive Behandlung erfahren haben, wobei besonders der Weg von den Wunderkammern der Renaissance und des Barock hin zur Herausbildung von Museen verstärkte

\footnotetext{
${ }^{71}$ Vgl. UniA GÖ, Kur., 5584, Schreiben Bartlings an das Universitätskuratorium, 5.2.1840.

72 Vgl. UniA GÖ, Kur. Pers., 4973, Schreiben Grisebachs an das Universitätskuratorium, 18.12.1866.

${ }^{73}$ UniA GÖ, Kur., 5590, Schreiben Bartlings an das Universitätskuratorium, 5.3.1837.

74 Vgl. Siemer 2004, S. 199 f.

75 Vgl. ebd., S. 200.
} 
Aufmerksamkeit erfuhr, tritt die Institutionalisierung universitärer Sammlungen davor zurück. ${ }^{76}$ Dabei gerät aus dem Blick, dass auch diese eine Öffnung und explizite Bildungsabsichten verfolgten. Herbarien als Forschungssammlungen der Botanik treten darüber hinaus wiederum gegenüber den botanischen Gärten stark in den Hintergrund, die bisher weitaus intensivere Beachtung in Forschungsarbeiten fanden. So wird bezüglich der botanischen Gärten durchaus eine Analogie zur sich herausbildenden Museumslandschaft im 18. und 19. Jahrhundert betont und auch hier eine Professionalisierung und zunehmende Öffnung für das 19. Jahrhundert festgestellt. ${ }^{77}$

Ein Blick auf die Gründungsgeschichte der Herbarien zeigt allerdings, dass auch sie sich in diese Entwicklung einfügen. So schreiben Hurka und Neuffer in einer der wenigen Veröffentlichungen zu diesem Thema, dass es sich auch bei Herbarien bis zum Ende des 18. Jahrhunderts vor allem um Privatsammlungen handelte, die nach dem Tod des*der Besitzers*in verkauft oder aufgelöst wurden. ${ }^{78} \mathrm{Im}$ 19. Jahrhundert habe dann die Institutionalisierung der großen Herbarien eingesetzt, die sich durch

„[...] wissenschaftliche Zweckbestimmung; öffentliche Zugänglichkeit und Ausbaumöglich-

keiten durch Zukauf oder Überlassung anderer Sammlungen sowie Tauschverfahren;

Leihverkehr zwischen wissenschaftlichen Einrichtungen [...]“79

und weitgehende Unabhängigkeit von privater Einflussnahme auszeichneten.

In der Herausbildung des Göttinger Herbariums zeigt sich dieses Wechselspiel zwischen Öffnung und Nutzbarmachung einerseits sowie Ausdifferenzierung und Spezialisierung andererseits beispielhaft. ${ }^{80}$ Seine Gründung ist durchaus als eine Fortführung der Bemühungen des Academischen Museums um Öffnung, Zugänglichkeit und Nutzbarmachung für Forschung und Lehre einzustufen. ${ }^{81}$ An genau diese Bestrebungen knüpfen Bartlings Argumentationen an. Gleichzeitig steht die Abgabe der Sammlungen an den botanischen Garten und die darauf folgende Institutionalisierung auch für die Ausdifferenzierung der Naturgeschichte und die Herausbildung neuer Disziplinen im 19. Jahrhundert, zu der auch die Einrichtung von Institutionen gehörte, die den neuen fachwissenschaftlichen

${ }^{76}$ Dabei sei aber explizit auf jene Stimmen hingewiesen, die sich entschieden gegen eine Engführung auf das 19. Jahrhundert als Gründungsepoche der modernen Museen aussprechen und auch für die Zeit vor 1789 eine Zugänglichkeit und Bildungsabsicht in vielen Sammlungen konstatieren. Vgl. Savoy 2015.

77 Vgl. Rieppel 2016; Vogel 2013, S. 270.

78 Vgl. Hurka \& Neuffer 2011, S. 151.

79 Vgl. ebd., S. 152.

${ }^{80}$ Dieses Wechselverhältnis arbeitet Dorinda Outram beispielsweise für das Muséum National d'Histoire Naturelle in Paris für den Zeitraum von 1780-1830 heraus. Vgl. Outram 1996.

81 Zur besonderen Rolle des Göttinger Museums im Verhältnis zur bürgerlichen Öffentlichkeit der Haupt- und Residenzstädte des alten Reiches und den dortigen Museumsgründungen vgl. François 2015, S. 54-55. 
Anforderungen und Bedürfnissen Rechnung trugen.82 Gleichzeitig - um an Cornelia Webers Anmerkungen zum Institutionalisierungsprozess akademischer Sammlungen anzuknüpfen - machte sich die Universität mit der Einrichtung eines öffentlichen Herbariums unabhängig von der persönlichen Ausstattung der Dozierenden. ${ }^{83}$ Erst durch die staatliche Vorsorge für universitäre Lehre und Forschung konnte sich die private Sammlungspraxis zu einer institutionellen wandeln. ${ }^{84}$ Dabei gehört das Göttinger Herbarium mit seiner Gründung im Jahr 1832 nicht ohne Grund zu den älteren Institutionen. ${ }^{85}$ Dass sich der dienstjüngere und zudem noch Privatdozent ${ }^{86}$ Bartling gegenüber dem diensthabenden Direktor Schrader durchsetzen konnte, liegt nicht zuletzt in dem von Schlumbohm beschriebenen intra- und interuniversitären Konkurrenzprinzip der Georgia Augusta begründet. ${ }^{87}$ Dieses hatte zum Ziel, „[...] die Stellung Göttingens im Wettbewerb mit den anderen Hochschulen zu stärken."88 Dabei dienten die richtungsweisenden Universitäten des Reichs und des europäischen Auslands als Maßstab, den es zu übertreffen galt. ${ }^{89}$ Die Herausbildung einer neuen Institution mit den Sammlungspraktiken, Öffnen“ und ,Nutzbarmachen' ist damit sowohl durch eine Verortung in die breitere Sammlungsgeschichte zu erklären als auch mit einem Blick auf die besonderen Standortvoraussetzungen und die Spezifika der Göttinger Universitätspolitik. Denn wie andere Sammlungen des beginnenden 19. Jahrhunderts ist auch das Göttinger Herbarium vor allem als eine Einrichtung der Regierung anzusehen, die unabhängig von privaten Ressourcen von Hannover aus verwaltet wurde. ${ }^{90}$

82 Vgl. Füssel 2015a, S. 57; Gaskell 2017, S. 9 f.

83 Vgl. Weber 2011, S. 101 f.

${ }^{84}$ Vgl. ebd., S. 112.

${ }^{85}$ Die meisten Herbarien wurden im Zeitraum von 1925 bis 2000 gegründet. Vgl. Hurka \&

Neuffer 2011, S. 152.

86 Vgl. Tütken 2005.

87 Vgl. Schlumbohm 2016.

88 Ebd., S. 252.

${ }^{89}$ Vgl. ebd., S. 254.

${ }^{90}$ Eine ähnliche Entwicklung beschreibt zum Beispiel Anke te Heesen für die Sammlungen der Gesellschaft Naturforschender Freunde zu Berlin um 1800. Vgl. te Heesen 2002. 


\subsection{Werte zuschreiben}

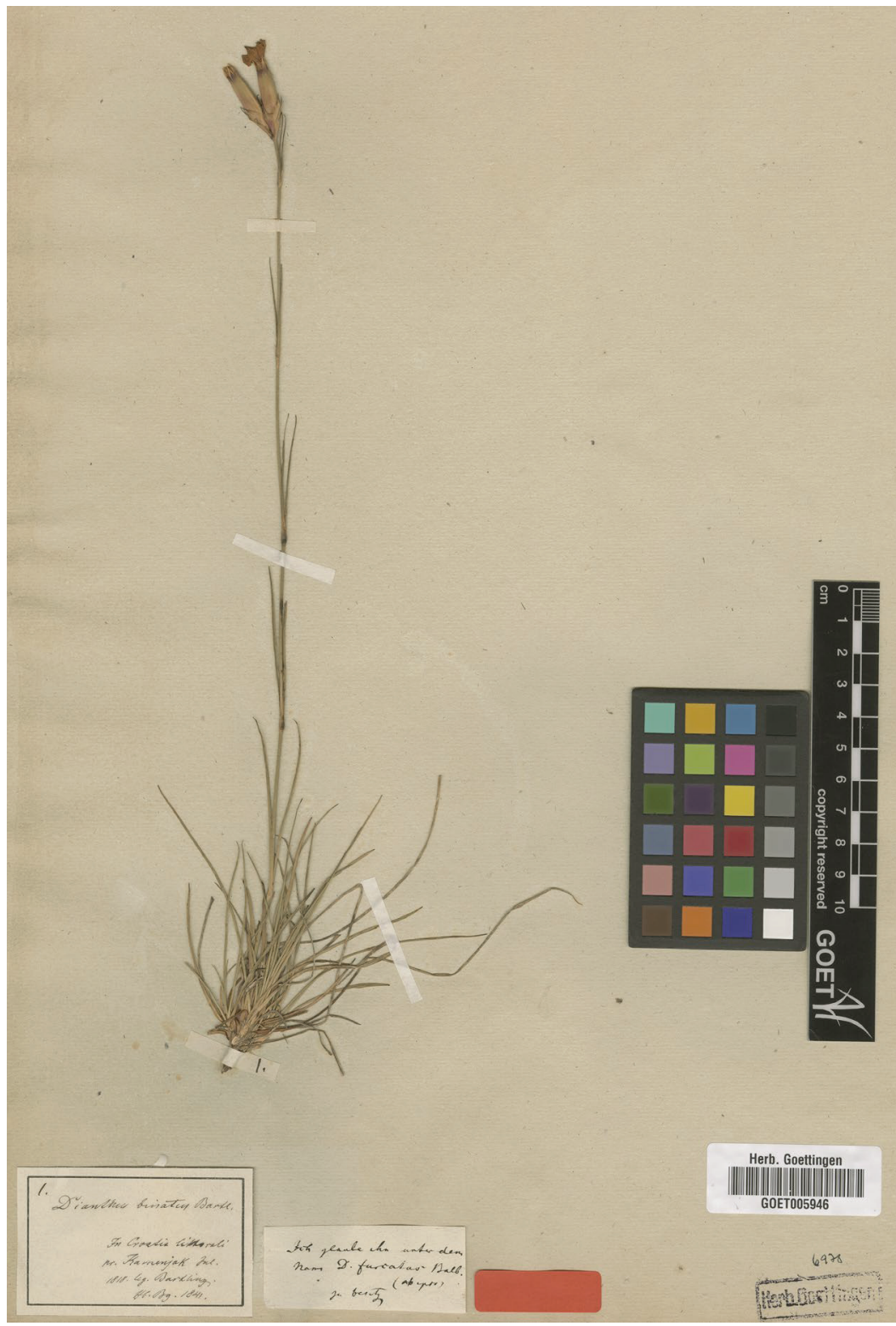

Abb. 4: Herbarbeleg von Dianthus caryophyllus L., gesammelt von F. G. Bartling im Juli 1818 nabe des Kap Kamenjak an der südlichen Spitze der Halbinsel Istrien, Herbarium Göttingen, GOET005946. 
Der Herbarbeleg der Pflanze Dianthus binatus Bartl. (Dianthus caryophyllus L.) zeigt beispielhaft, wie Pflanzenbelege in der Anfangsphase des Göttinger Herbariums angefertigt worden sind. Lediglich zwei der vier Papierstreifen zum Fixieren der Pflanze, der rote Aufkleber, der den Beleg der Typussammlung zuordnet, der Barcode mit Inventarnummer, der Stempel des Herbariums, der Farbkeil und das Maßband sowie die vierstellige mit Bleistift notierte Nummer, die auf ein altes Datenbankensystem verweist, können vorbehaltlos als nicht dem Ausgangsmaterial zugehörige Bestandteile des Belegs benannt werden. Von wann und von wem der auf einem zusätzlichen Etikett notierte Kommentar ,Ich glaube ihn unter dem Namen D. furcatus Balb. [...] zu besitzen' stammt, ist unklar. Während viele der präparierten Pflanzen des Herbariums aber mit der Zeit auf neues, säurefreies Papier umgebettet worden sind, ist bei Dianthus binatus. das Originalmaterial erhalten. Indem der Beleg zu einem Großteil noch aus seinen ursprünglichen Bestandteilen besteht, ermöglicht er es ganz besonders, die Praxis der wissenschaftlichen Objektanfertigung am Göttinger Herbarium in der Zeit von 1832 bis 1852 nachzuvollziehen.

Schon indem zwei der klebenden Papierstreifen ersetzt wurden, tritt das Ausgangsmaterial deutlich hervor. Auch verweist einer der beiden alten Streifen, versehen mit der Kennziffer ,I.', direkt auf das der präparierten Pflanze zugehörige Etikett. Dieses ist durch einen Vergleich mit anderen Herbarbelegen Bartlings ebenfalls als Original auszuweisen. Mit einem in schwarz gezeichneten Rahmen versehen, trägt das Etikett folgende Angabe: In Croatia littorali pr. Kamenjak Jul. 1818. leg. Bartling. Hb. Bg. 1841.' Die Nelke wurde demnach im Juli 1818 nahe des an der südlichen Spitze der Halbinsel Istrien gelegenen Kap Kamenjak von dem Göttinger Botaniker Bartling gesammelt. Der Hinweis ,Hb. Bg. 1841' ist an dieser Stelle besonders interessant, da er mit großer Wahrscheinlichkeit auf den Ankauf des Herbariums Bartlings am 23. August 1841 verweist. Somit ist davon auszugehen, dass die Pflanze auch nach den von Bartling aufgestellten und mit dem Kuratorium vereinbarten Kriterien zur Anfertigung von Herbarbelegen fixiert worden ist. Denn sicherlich legte der Vorsteher des Herbariums großen Wert darauf, seine Sammlung vor Aufnahme in dasselbe in die den Instruktionen entsprechende, ,richtige' Form zu bringen. 


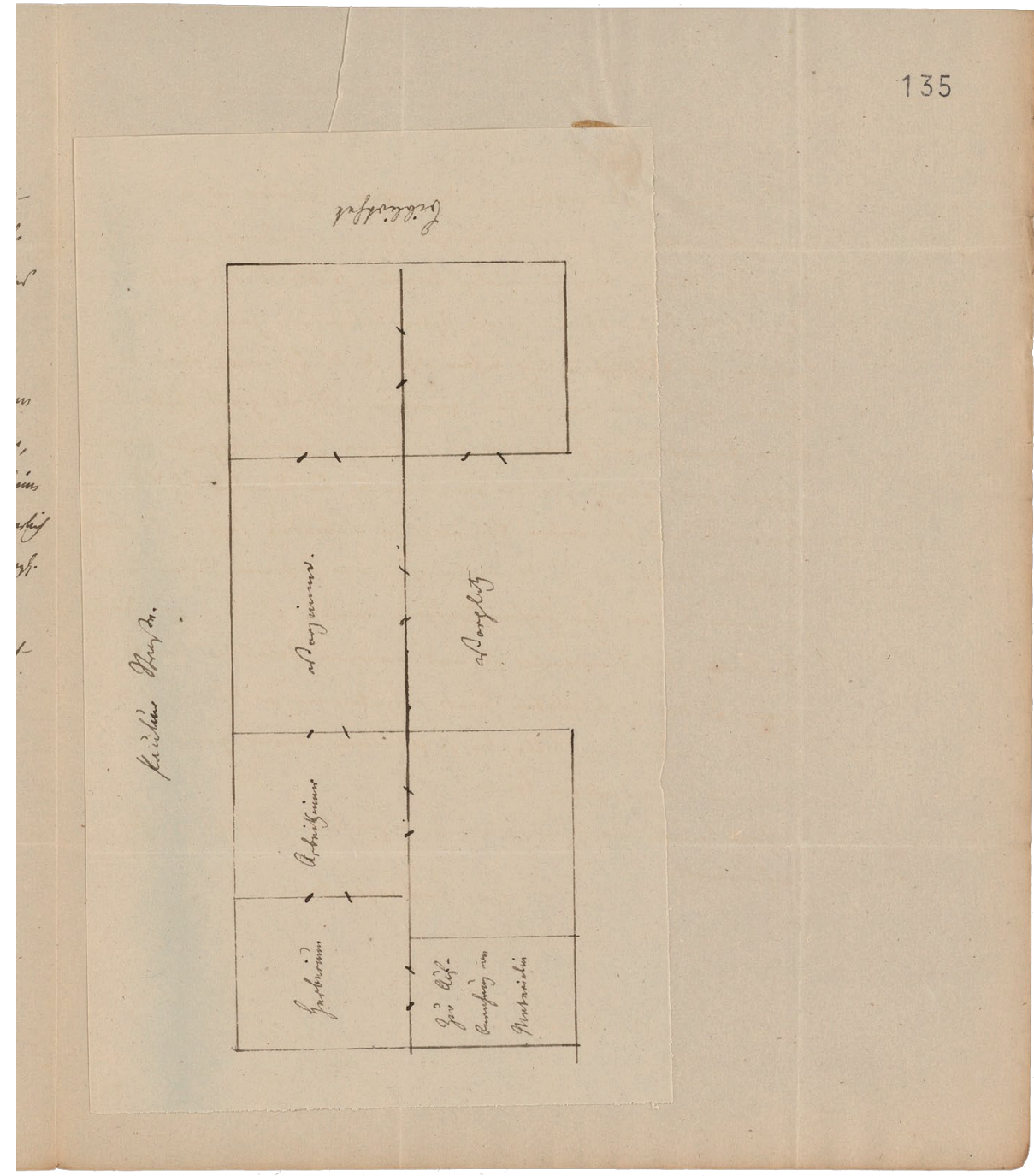

Abb. 5: Einem Schreiben F. G. Bartlings vom 21. Dezember 1833 an den Prorektor und den Senat der Universität beigefügte Zeichnung, Universitätsarchiv Göttingen, UniA GÖ, Kur., 6912.

Einem Schreiben vom 21. Dezember 1833, das vermutlich an den Prorektor und den Senat der Universität gerichtet war, fügte Bartling eine Zeichnung bei. Sie ist an einer Ecke mit Klebstoff versehen, auf dem Papierbogen fixiert worden und zeigt einen in Tinte angefertigten Grundriss. Die abgebildete Etage des länglichen Gebäudes besitzt sieben Zimmer, die sich in U-Form um einen als ,Vorplatz 
bezeichneten Raum anordnen. Oberhalb der Zeichnung wurde die Paulinerstraße vermerkt und an der rechten Seite die Bibliothek. Der Plan ist demnach nicht nach Norden, sondern nach Süden ausgerichtet. Von den Räumen haben vier eine Beschriftung erhalten. Das größere nach Osten ausgerichtete Zimmer trägt die Bezeichnung ,Herbarium‘. Der im Westen daran angrenzende Raum wird als ,Arbeitszimmer ${ }^{6}$ ausgewiesen, während der im Süden angrenzende ,zur Aufbewahrung von Materialien' heißt. Südlich an den Vorplatz schließt das ,Vorzimmer an. Außerdem wurden acht Türen eingezeichnet. Auf Treppen oder Fenster gibt es allerdings keine Hinweise. Auch einen Maßstab lässt der Plan vermissen.

Während die Zeichnung selbst keine weiteren Informationen bereithält, verdeutlicht das Schreiben sehr wohl, um welches Gebäude es sich handelt und in welchem Kontext der Plan erstellt worden ist. Gezeigt wird die Beletage des sogenannten ,Meisterschen Hauses'. Dabei handelte es sich um das ehemalige Wohnhaus Paulinerstraße Nummer 21 des Rechtsgelehrten und geheimen Justizrats Georg Jacob Friedrich Meister (1755-1832). ${ }^{91}$ Es grenzte im Westen an das Bibliotheksgebäude und im Osten an ein weiteres Professorenhaus an. ${ }^{92}$ Nach dem Tod Meisters wurde es am 18. Februar 1833 vom Kuratorium für die Universität angekauft. ${ }^{93}$ Die gängige Bezeichnung des Hauses als Auditoriengebäude unterschlägt allerdings, dass einige Räumlichkeiten - namentlich jene im ersten Stock - zumindest für den Zeitraum von 1834 bis 1844 für andere Zwecke genutzt worden sind. ${ }^{44}$ So zeugt das Schreiben Bartlings von dem nach dem Kauf einsetzenden Aushandlungsprozess zwischen Kuratorium, Prorektor und Senat, dem Landbauinspektor Praël sowie den unterschiedlichen Antragstellenden bezüglich der Gebäudenutzung und Raumverteilung. Denn zunächst wurden nur die Räumlichkeiten im Erdgeschoss und im zweiten Stock zu Auditorien umgebaut. Um die Zimmer im ersten Stock bemühten sich unterschiedliche Interessenten, die in Schreiben Nutzungsvorschläge und Argumente formulierten. $\mathrm{Zu}$ diesen gehörte auch Bartling, der in verschiedenen Schreiben für eine Raumbelegung durch das Herbarium argumentierte. Sein Vorschlag vom 21. Dezember 1833, dem er diese Zeichnung beifügte, wurde schließlich zur Umsetzung gebracht. Im Juni 1834 zog die Sammlung in das Meistersche Haus um. ${ }^{95}$

\footnotetext{
${ }^{91}$ Vgl. Oberdiek 2002, S. 43.

${ }^{2}$ Das angrenzende Professorenhaus gehörte dem geheimen Justizrat Arnold Hermann Ludwig Heeren (1760-1842).

93 Vgl. UniA GÖ, Kur., 6912, Kaufkontrakt, 18.2.1833.

${ }^{94}$ Neben dem Herbarium waren auch die Zeichenakademie des Prof. Carl Wilhelm Friedrich Oesterley (1805-1891) sowie die Singakademie des Musikdirektors Johann August Günther Heinroth (1780-1846) in der Beletage untergebracht. Vgl. UniA GÖ, Sek., 528, Schreiben des Universitätskuratoriums an den Prorektor und den Senat, 3.2.1834. Im Jahr 1844 erteilte das Kuratorium der Universität die Genehmigung, das Meistersche und das Heerensche Haus zu öffentlichen Auditorien umzubauen. Vgl. UniA GÖ, Sek., 528, Schreiben des Kuratoriums an die Universität, 16.10.1844.

95 Vgl. UniA GÖ. Kur., 5584, Notiz vom Juni 1834.
} 
Doch welchen Weg ist das Herbarium in den Jahren von 1832 bis 1852 gegangen? Wie muss man sich die neu gegründete Institution vorstellen? Wie stand es um Zugänglichkeit und Nutzungsmöglichkeiten kurz nach 1832? Konnte der Wunsch nach Öffnung durch das Aufbrechen der Privatsammlung umgesetzt werden? Um diese Fragen beantworten zu können, muss sich zunächst vor Augen geführt werden, dass mit der Institutionalisierung der Sammlung auch eine Spezialisierung und Ausdifferenzierung stattfand, die wiederum mit einem gesteigerten Anforderungsprofil an die Sammlungsobjekte sowie die zur Aufstellung gedachten Räumlichkeiten einherging. Die Zeit von 1832 bis 1852 ist in diesem Zusammenhang als Etablierungsphase des Herbariums zu verstehen, in der die Bedürfnisse und die Infrastruktur dieser neuen Einrichtung ausgehandelt wurden.

Der gesteigerten Anspruchshaltung war es dabei auch zu verdanken, dass sich das Herbarium in den ersten Jahren seines Bestehens, von 1832 bis 1834, zunächst im Wohnhaus seines Gründers in der Roten Straße 239 (heute Nummer 32) befand. ${ }^{96}$ In einem Zimmer im ersten Stock, das südwärts zum Hof gelegen war, wurde die Sammlung aufgestellt und von Bartling so geordnet und in Stand gesetzt, dass sie schließlich auch zur allgemeinen Benutzung freigegeben werden konnte. ${ }^{97}$ In einem solchen Zustand befanden sich die Sammlungen der Universität laut Bartling bis dahin nicht. Seiner Meinung nach seien „,[...] einige Jahre erforderlich [...], um die Sammlung zu einem solchen Grade von Vollständigkeit zu bringen, daß sie sich zu einem öffentlichen Institute der Universität qualificiert $[\ldots]^{698}$. Aus diesem Grund wurde in der Verordnung über die Benutzung des Herbariums auch festgehalten, dass dieses erst dann zugänglich gemacht werden soll, wenn ein solcher Zustand auch erreicht sei. ${ }^{99}$

Der Wunsch nach Zugänglichkeit und Nutzbarmachung der Sammlung stand demnach von Beginn an eng mit einem Anspruch auf Wertigkeit in Verbindung. Dieser führte dazu, dass dem Öffnungsprozess der Sammlung zunächst eine regelrechte Schließung vorausging. ${ }^{100}$ Besonders von Seiten Bartlings ist diese Anspruchshaltung vielfach nachweisbar. Bereits in seinen Vorschlägen zur Gründung eines Herbariums machte er deutlich, welche Kriterien eine

\footnotetext{
96 Vgl. StadtA GOE, B 100 Nr. 293; StadtA GÖ, IV A 1, Personal-Bestand der Georg-AugustUniversität zu Göttingen. Auf das halbe Jahr von Michaelis 1832 bis Ostern 1833, S. 4.

${ }^{7}$ Vgl. UniA GÖ, Kur., 5584, Schreiben Bartlings an den Kanzleirat, 10.4.1832; UniA GÖ, Kur., 5584, Schreiben Bartlings an das Universitätskuratorium, 14.11.1832, Anlage C, Vertrag zwischen Maurermeister H. C. Linne und F. G. Bartling, 13.11.1832.

98 UniA GÖ, Kur., 5584, Schreiben Bartlings an das Universitätskuratorium, 9.9.1832.

${ }^{99}$ Vgl. UniA GÖ, Kur., 5584, Schreiben Bartlings an das Universitätskuratorium, 14.11.1832, Anlage A, B, Von der Benutzung des Universitätsherbarii.

100 Anne Mariss weist zum Beispiel darauf hin, dass sich die Privatsammlungen häufig in einem besseren Zustand befanden als die sich herausbildenden Sammlungen der Universität. Mit der Institutionalisierung einer Sammlung sei deshalb auch nicht zwangsläufig eine qualitative Verbesserung einhergegangen. Vgl. Mariss 2018, S. 216 f.
} 
„[...] Sammlung [...] von wahrem wissenschaftlichem Werthe [...]“101 seiner Meinung nach zu erfüllen habe. ${ }^{102}$ Vor allem Vielfalt und Vollständigkeit des Pflanzenmaterials waren laut Bartling notwendige Voraussetzungen für den Nutzen der Sammlung. ${ }^{103}$ Darüber hinaus wurden die Zurichtung und ganz besonders die Bezeichnung und Beschriftung der Belege von ihm als Qualitätsmaßstäbe benannt. ${ }^{104}$ Erst wenn die Universitätssammlung diese „[...] aus dem jetzigen Stande der Botanik hervorgehenden Anforderungen [...]“105 erfülle, könne sie für die Wissenschaft nutzbar gemacht werden. Vor diesem Hintergrund wird auch verständlich, warum Bartling, anders als Schrader, dem Altbestand an Sammlungen aus dem Academischen Museum eine weitaus geringere Bedeutung beimaß:

„So schätzbar und wichtig sie auch in mehrfacher Beziehung seyn mögen, so instructiv sie auch in einzelnen Fällen seyn können, so bieten sie doch weder die erforderliche Vollständigkeit des Materials, noch die nothwendigen Belege zu den Fortschritten und dem heutigen Zustande der in den neuern Zeiten mit so großem Erfolge bearbeiteten Wissenschaft, da sie nur die Flora einiger weniger Länder enthalten und größtentheils in einer Zeit gemacht sind, in welcher die Pflanzenkunde noch weit von dem Grade von Ausdehnung und Genauigkeit entfernt war, den sie gegenwärtig erreicht hat [...].“106

Einer der ersten mit der Gründung des Herbariums einhergehenden Schritte war deshalb die Formulierung verschiedener Regularien und Instruktionen, die eine rechtliche beziehungsweise vertragliche Festlegung der gewünschten Standards darstellten. Diese Regularien wurden von Bartling am 14. November 1832 verfasst und anschließend dem Kuratorium zur Prüfung und Absegnung unterbreitet. ${ }^{107}$ Sie bestimmten detailliert die Art der Einrichtung, Benutzung und Verwaltung der Sammlung.

In einem ersten Schritt wurde festgelegt, dass die Sammlung in verschiedene Abteilungen einzuteilen sei. ${ }^{108} \mathrm{Zu}$ diesen gehörte das allgemeine Herbarium als

101 UniA GÖ, Kur., 5584, Schreiben Bartlings an das Universitätskuratorium, 8.12.1830.

102 Zur Verdeutlichung sandte Bartling dem Kuratorium gemeinsam mit seinem Schreiben sogar eine kleine exemplarische Sammlung zu. Aus welchen Pflanzenbelegen diese bestand, ist jedoch nicht mehr zu rekonstruieren. Vgl. UniA GÖ, Kur., 5584, Schreiben Bartlings an den Kanzleirat, 22.10.1831.

103 Vgl. UniA GÖ, Kur., 5584, Schreiben Bartlings an das Universitätskuratorium, 9.9.1832.

104 Vgl. UniA GÖ, Kur., 5584, Schreiben Bartlings an das Universitätskuratorium, 8.12.1830; UniA GÖ, Kur., 5584, Schreiben Bartlings an das Universitätskuratorium, 9.9.1832.

${ }^{105} \mathrm{Ebd}$.

${ }^{106}$ Ebd.

107 Vgl. UniA GÖ, Kur., 5584, Schreiben Bartlings an das Universitätskuratorium, 14.11.1832. Das Kuratorium genehmigte den Vorschlag Bartlings in einem Schreiben vom 4. Dezember 1832. Vgl. UniA GÖ, Kur., 5584, Schreiben des Universitätskuratoriums an Bartling, 4.12.1832.

108 Dieser Gliederung übergeordnet wurde außerdem zwischen dem Universitäts-Herbarium und einem Garten-Herbarium unterschieden. Letzteres sollte in der Nähe des Gartens aufgestellt 
Hauptsammlung, eine Sammlung der im Königreich einheimischen Gewächse, eine Sammlung der offiziellen Gewächse, eine Sammlung der im Forstwesen, der Ökonomie, der Medizin und anderen Fachrichtungen relevanten Gewächse sowie eine Abteilung der nicht täglich mit den vier ersten Abteilungen zu verbindenden Sammlungen. ${ }^{109}$ Damit sollte zum einen die Benutzung erleichtert werden, zum anderen wurde aber auch eine Abstufung in der Wertigkeit vorgenommen. So handelte es sich bei der Hauptsammlung um die kostspieligste der fünf Abteilungen, die auch einer intensiveren Schonung, Beaufsichtigung sowie Bearbeitung bedurfte. ${ }^{110}$ Pflanzenexemplare sollten erst in die übrigen Abteilungen gelangen, wenn sie nicht in das Hauptherbarium einzuordnen waren. Seine Vervollständigung und Erweiterung war derjenigen der übrigen Abteilungen vorzuziehen. ${ }^{111}$ Auch durften Exemplare - sofern sie nicht von bedeutenden Botaniker*innen stammten - erst dann in die Hauptsammlung aufgenommen werden, nachdem sie sorgfältig untersucht und bestimmt worden waren. ${ }^{112}$

Die neuen Wertmaßstäbe, die an die Sammlung gerichtet wurden, treten noch einmal deutlicher hervor, stellt man den ersten vier Abteilungen die fünfte Abteilung kontrastierend gegenüber. Denn dieser fielen all jene Pflanzensammlungen zu, die den neuen Standards der ,[...] eigenthümlichen Art ihrer Anordnung wegen, oder weil sie abgeschlossene Werke ausmachen, oder aus

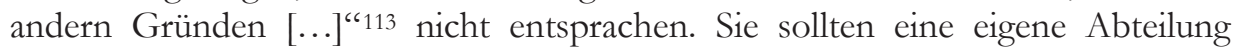
erhalten, da sie sonst an wissenschaftlichem Wert eingebüßt hätten. ${ }^{114}$ Was genau damit gemeint war, zeigt sich, wenn man einen intensiveren Blick auf die Instruktionen zur Anlegung der Abteilungen und der darin enthaltenen Herbarbelege wirft, von denen die fünfte Abteilung bewusst ausgeschlossen wurde. ${ }^{115}$

In diesen Anweisungen wurde dargelegt, wie die Präparate im Detail anzufertigen und aufzubewahren seien: Die Exemplare mussten auf weißem, 20 Zoll hohem und 24 Zoll breitem Papier fixiert, die Flechten in Pappkästchen und die Drogen in Gläsern aufbewahrt werden. ${ }^{116}$ Die einzelnen Belege sollten so zugerichtet werden, dass pro Papierbogen ausschließlich ein Pflanzenexemplar befestigt wird. Auch wurde festgelegt, dass jeder Bogen mit einem nummerierten Etikett zu versehen ist, das den Namen der Art und des Exemplars,

werden und alle dort kultivierten Arten belegen. Vgl. UniA GÖ, Kur., 5586, Schreiben Bartlings an das Universitätskuratorium, 17.2.1837; Wagenitz 2016, S. 224.

109 Vgl. UniA GÖ, Kur., 5584, Schreiben Bartlings an das Universitätskuratorium, 14.11.1832,

Anlage A, A, Von der Einrichtung des Universitäts-Herbarii.

110 Vgl. UniA GÖ, Kur., 5584, Schreiben Bartlings an das Universitätskuratorium, 14.11.1832.

111 Vgl. ebd.

112 Vgl. ebd.

113 Ebd.

114 Vgl. UniA GÖ, Kur., 5584, Schreiben Bartlings an das Universitätskuratorium, 14.11.1832.

115 Vgl. UniA GÖ, Kur., 5584, Schreiben Bartlings an das Universitätskuratorium, 14.11.1832, Anlage A, A, Von der Einrichtung des Universitäts-Herbarii.

116 Wenn Bartling in der Instruktion von ,Exemplaren' spricht, bezieht er sich vermutlich im Speziellen auf die Gefäßpflanzen. 
Informationen zum*zur Sammler*in sowie das Zugangsdatum trägt. Die Exemplare einer Art sollten in Umschlägen aus starkem blauem Papier aufbewahrt werden, auf denen der Name der Gattung zu verzeichnen ist. Darüber hinaus waren die Sammlungen in dauerhaften Pappdeckeln und in verschlossenen Schränken aufzubewahren und nach einem gebräuchlichen System so zu ordnen, dass die einzelnen Gattungen und Arten möglichst schnell aufzufinden sind. Für die systematische Aufstellung wurde vorläufig die von Bartling 1830 herausgegebenen Ordines naturales plantarum ${ }^{117}$ zurate gezogen. ${ }^{118}$ Und in diese systematische Anordnung schienen die der Abteilung fünf zufallenden Sammlungen laut Bartling nicht eingefügt werden zu können - zumindest nicht auf solche Weise, dass sie wissenschaftlich nutzbar zu machen waren. ${ }^{119}$

Dieser Einschätzung Bartlings liegt ein Wertverständnis botanischer Sammlungen zugrunde, das ganz offensichtlich als Fortführung der Bestimmungen Carl von Linnés zur Anlegung eines Herbariums zu verstehen ist, die dieser in seiner 1751 veröffentlichten Philosophia botanica formulierte. ${ }^{120}$ Diese Vorschriften hatten, wie Staffan Müller-Wille es zusammenfasst,

„[...] eine Standardisierung des Herstellungsverfahrens [zum Ziel], die garantieren konnte, daß Eigentümlichkeiten der gesammelten Pflanzen nicht dem aquirierenden [sic!] Prozeß zu schulden waren, oder zumindest sicherstellte - wenn sich solche Eigentümlichkeiten, etwa durch das Trocknen und Einkleben, schon nicht vermeiden ließen -, daß diese Eigentümlichkeiten immer auf dieselbe Weise zustande kamen.“121

Neu war hierbei die Anweisung Linnés, nur eine Pflanze pro Blatt aufzukleben und die einzelnen Blätter nicht wie bei früheren Herbarien noch üblich zusammen zu heften. ${ }^{122}$ Wie Müller-Wille an Linnés Herbarschrank beispielhaft ausführt, gründete diese Form der Anlegung eines Herbariums auf dem Wunsch, die einzelnen Herbarbelege stets in Bewegung halten zu können. ${ }^{123}$ Denn einen Wert erhielt die einzelne Pflanze erst dann, „[...] wenn sie sich in einer reproduktiven und zirkulatorischen Bewegung als äquivalent zu anderen Pflanzen erwies und in das Tableau universeller, taxonomischer Beziehungen eintrat." So weist Müller-Wille darauf hin, dass die Aufforderung Linnés, sich um die ,Zahl der Arten' zu bemühen, eben nicht einfach als Aufruf zur Vermehrung der

\footnotetext{
117 Vgl. Bartling 1830.

118 Vgl. UniA GÖ, Kur., 5584, Schreiben Bartlings an das Universitätskuratorium, 14.11.1832.

119 An dieser Stelle kann nur spekuliert werden, ob beispielsweise auch der Altbestand aus dem Academischen Museum in die fünfte Abteilung des Herbariums fiel. Bartlings Einschätzungen zu diesen Sammlungen legen eine solche Vermutung jedenfalls nahe.

${ }^{120} \mathrm{Vgl}$. Linné 1751, \$11, S. 7.

121 Müller-Wille 2001, S. 25.

122 Vgl. ebd., S. 26.

${ }^{123}$ Vgl. ebd.

${ }^{124}$ Ebd., S. 37.
} 
Sammlung zu verstehen sei. ${ }^{125}$ Vielmehr müsse nach Linné der Erweiterung der Sammlung eine genaue Bestimmung der Arten vorausgehen. Das taxonomische Tableau stellte demnach nur auf den ersten Blick eine endgültige Anordnung dar, während es sich in der Praxis vielmehr durch ein ständiges Neuordnen auszeichnete. ${ }^{126}$

In diesem Zusammenhang müssen auch die Wünsche Bartlings zu der Anlegung der Herbarbelege sowie der Aufstellung der Sammlung gesehen werden. Nicht ohne Grund spricht er lediglich von einer ,vorläufigen Anordnung' im Herbarium. In dem Schreiben, das er den als Vorschlag vorgelegten Instruktionen beifügte, machte Bartling deutlich, dass die Klassifikation der Gewächse ständigen Veränderungen unterworfen sei und deshalb keine endgültige Entscheidung über die Anordnung der Sammlung getroffen werden könne. ${ }^{127}$ Aus diesem Grund wurden die Pflanzenexemplare wie beispielsweise auch Dianthus binatus auf einzelnen Papierbögen fixiert und erst nach einer genauen Beschreibung in das Herbarium eingeordnet. Auf diese Weise blieben sie isoliert und flexibel bezüglich ihrer Platzzuweisung in der Sammlung.

Neben der Herrichtung der Belege selbst und der Aufstellung der Sammlung wurden außerdem eine gewissenhafte Katalogführung sowie genaue Nutzungsbedingungen festgelegt. ${ }^{28}$ Die ständige Anwesenheit des Vorstehers oder des Gehilfen des Herbariums während dessen Öffnungszeiten sollte beispielsweise sicherstellen, dass die bestehende Ordnung nicht gestört würde. Ein eigenmächtiges Handeln in der Sammlung war den Besucher*innen nicht gestattet. Pflanzenbelege durften allein von den Mitarbeitenden aus den Schränken genommen und wieder einsortiert werden. Kontrollierende Instanz gegenüber dem Vorsteher des Herbariums war wiederum das Kuratorium. Diesem musste der Vorsteher nicht nur Verzeichnisse über hinzukommende und abgegebene Pflanzen sowie über die Einnahmen und Ausgaben einreichen, sondern in regelmäßigen Berichten auch über alle das Herbarium betreffenden Angelegenheiten Rechenschaft ablegen. ${ }^{129}$ Auf diese Weise wurde auf verschiedenen Ebenen Kontrolle ausgeübt, um die Einhaltung der formulierten Qualitätsmaßstäbe in der Praxis abzusichern. Gleichzeitig drückte sich in diesen die ,innere Struktur ${ }^{6}$ der Sammlung betreffenden Instruktionen der Wunsch nach einer langfristigen Erhaltung der Sammlung aus.

Während die erste Phase des Göttinger Herbariums von der Herstellung einer grundlegenden Infrastruktur geprägt war, zeugt der beschriebene Plan von 1833

\footnotetext{
125 Vgl. ebd.

126 Vgl. ebd., S. 23.

127 Vgl. UniA GÖ, Kur., 5584, Schreiben Bartlings an das Universitätskuratorium, 14.11.1832.

128 Vgl. UniA GÖ, Kur., 5584, Schreiben Bartlings an das Universitätskuratorium, 14.11.1832, Anlage A, B, Von der Benutzung des Universitätsherbarii.

${ }^{129}$ Vgl. UniA GÖ, Kur., 5584, Schreiben Bartlings an das Universitätskuratorium, 14.11.1832, Anlage A, C, Von der Verwaltung des Universitäts-Herbarii; UniA GÖ, Kur., 5584, Schreiben des Universitätskuratoriums an Bartling, 4.12.1832.
} 
davon, dass kurz nach der Unterbringung der Sammlung in der Roten Straße auch die Frage nach angemessenen und zweckmäßigeren Räumlichkeiten ins Blickfeld rückte. Die Verhandlungen hinsichtlich der Zimmer im ehemals Meisterschen Haus an der Paulinerstraße (Abb. 6 \& Abb. 7), die im Sommer 1834 bezogen wurden, verdeutlichen, dass mit der Aufwertung der Sammlung auch das Erfordernis nach einer ihren Bedürfnissen entsprechenden Lokalität einherging. Bartlings Schreiben, in denen er seine Wünsche bezüglich einer möglichen Raumverteilung darlegt, geben genau Aufschluss darüber. ${ }^{130}$

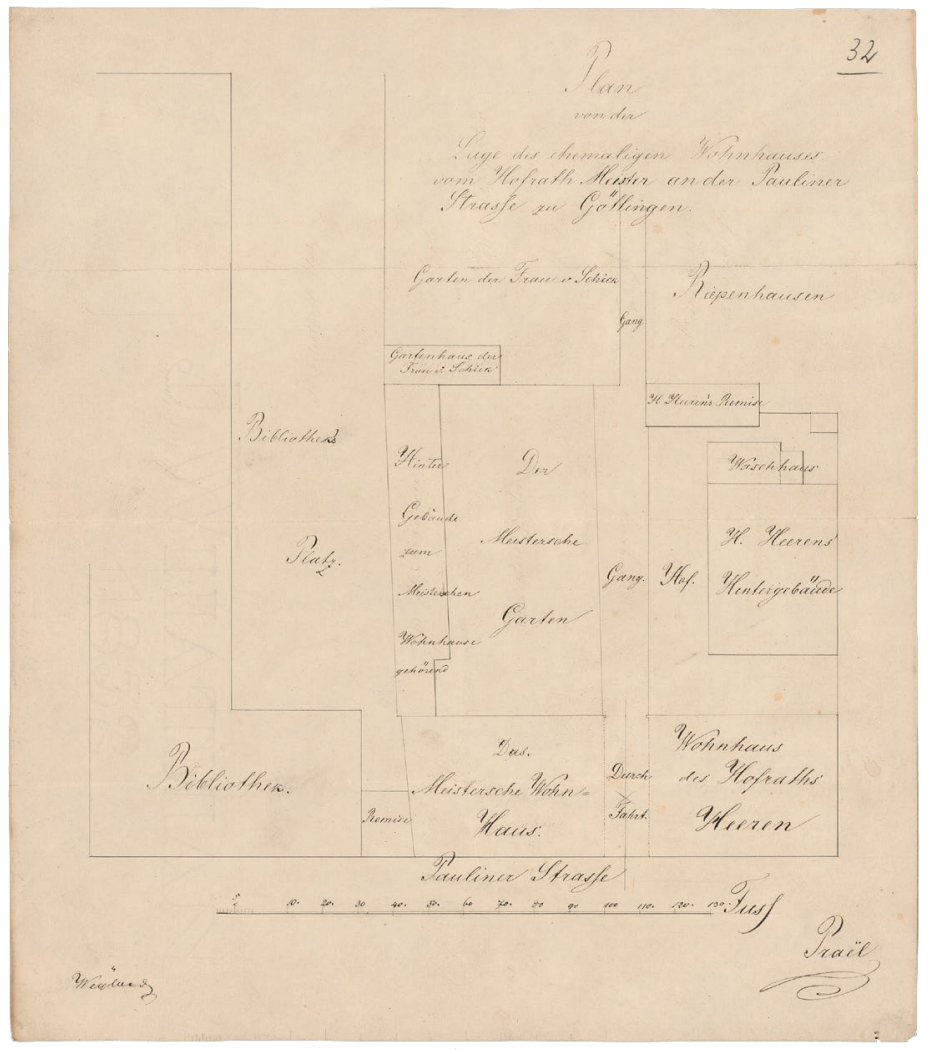

Abb. 6: Einem Schreiben Landbaninspektors Otto Prä̈l vom 24. März 1833 (UniA GÖ, Kur., 6912) an das Kuratorium beigefügte Zeichnung vom Landbauconducteur Weyland, Plan von der Lage des ehemaligen Wohnhauses vom Hofrat Meister an der Paulinerstraße ₹u Göttingen, Universitätsarchiv Göttingen, UniA GÖ, Kart., 3.

130 Vgl. UniA GÖ, Kur., 6912, Schreiben Bartlings an das Universitätskuratorium, 28.10.1833; UniA GÖ, Kur., 6912, Schreiben Bartlings an den Prorektor und den Senat, 21.12.1833. 


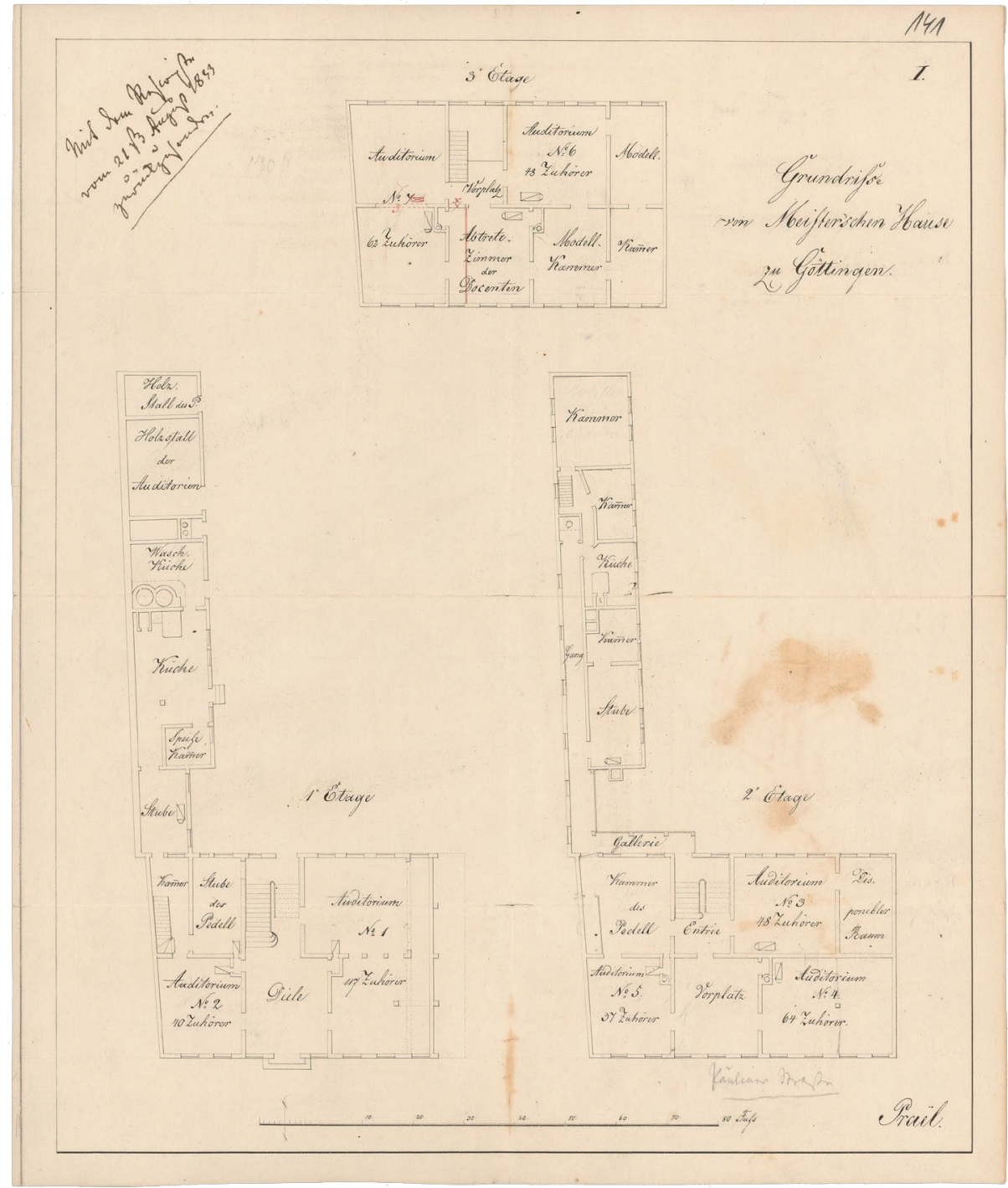

Abb. 7: Einem Schreiben des Landbauinspektors Otto Praël vom 8. Januar 1834 (UniA GÖ, Kur., 6912) an das Kuratorium beigefügte Zeichnung, Grundriss vom Meisterschen Hause ₹u Göttingen, Universitätsarchiv Göttingen, UniA GÖ, Kart., 3.

Bereits bei der Auswahl der Räume wurde mit Blick auf klimatische Bedingungen argumentiert. Denn Bartling favorisierte die nach Süden gerichteten, trockenen, luftigen und hellen Zimmer für die Sammlung. Sie boten seiner Meinung nach die geeigneten Voraussetzungen für eine bestmögliche 
Konservierung der Pflanzenpräparate. Dass er sich gegen die nach Westen ausgerichteten Räumlichkeiten entschied, ist dann mit der gewünschten Raumnutzung zu erklären: Neben einem Raum für die Hauptsammlung erachtete Bartling ein Arbeitszimmer sowie einen Lagerraum für notwendig. Da der Westflügel lediglich zwei Räume beherbergte und das Vorzimmer keine umfassende Nutzung ermöglichte, sah sein Plan die Zimmer im Ostflügel für das Herbarium vor. Das zum Hofe gelegene, dunklere Zimmer sollte zur Aufbewahrung der Papiervorräte und anderer Materialien genutzt werden. Der größte Raum war für die Sammlung selbst bestimmt. Das zweitgrößte Zimmer wurde zum Arbeitsraum und Aufbewahrungsort noch nicht bearbeiteter Pflanzen bestimmt. Neben den Lichtverhältnissen und dem Raumklima war jedoch auch die Größe bei der Verteilung der Zimmer von großer Bedeutung, schließlich sollte der Ausbau der Sammlung ermöglicht werden und der Raum für eine längere Zeitspanne ausreichen. Die erhaltenen Inventarien des Herbariums zeigen auch, dass mit dem Umzug in das Meistersche Haus von diesem Zugewinn an Fläche Gebrauch gemacht wurde. Denn die Grundausstattung aus den Jahren von 1832 bis 1834, die aus einem zweitürigen Tannenholzschrank, einem Schrank mit Glasscheiben für die Aufbewahrung der Flechten- und Drogensammlung, einem Arbeitstisch mit zwei Schubladen sowie Pappkästchen und Gläsern bestand, wurde allmählich ausgebaut. ${ }^{131} 1852$ umfasste die Einrichtung der Sammlung schließlich insgesamt sechs Schränke, von denen drei mit Glastüren versehen waren, zwei Arbeitstische, zwei Repositorien und drei Rohrstühle. ${ }^{132}$ Darüber hinaus führte das Inventarium 217 Gläser zur Aufbewahrung der Drogen, 30 Futterale für den Katalog, eine Papierschere, ein Federmesser, zwei Pinzetten und eine Drahtpresse auf. Die Sammlung wuchs an und nahm damit einhergehend zunehmend Platz und eine ausdifferenziertere Ausstattung in Anspruch. ${ }^{133}$

In den Jahren von 1832 bis 1852 wanderten die botanischen Sammlungen der Universität also zunächst als ungeordnetes Konglomerat von der Offizialwohnung des Direktors in die Räumlichkeiten eines Privatgebäudes in der Roten Straße. In einer zweiten Phase erhielt das Herbarium dann drei Zimmer in einem Universitätsgebäude an der Paulinerstraße, bis es am 7. Juni 1852 in den

131 Vgl. UniA GÖ, Kur., 5584, Schreiben Bartlings an das Universitätskuratorium, 14.11.1832, Anlage B, Veranschlagung der für das Universitäts-Herbarium vom 1ten November 1832 bis dahin 1833 zu verwendenden Ausgaben; UniA GÖ, Kur., 5584, Veranschlagung der für das Universitäts-Herbarium vom 1ten July 1833 bis ultimo Juny 1834 zu verwendenden Ausgaben.

132 Vgl. UniA GÖ, Kur., 5592, Rechnung über Einnahme \& Ausgabe für das Universitäts-

Herbarium zu Göttingen vom 1. Juli 1851 bis dahin 1852, 16.11.1852.

133 Dass die Sammlung in Hinblick auf ihre Einrichtung auch in den nachfolgenden Jahrzehnten weiter ausgebaut wurde, zeigt schließlich das 1875 begonnene ,Inventarium des UniversitätsHerbarium zu Göttingen', das erheblich umfangreicher als die ihm vorausgegangenen Exemplare ausfällt. Vgl. PWI.Syst.Geobotan.HV11. 
botanischen Garten zurückkehrte. ${ }^{134}$ Damit etablierte sich das Herbarium in den ersten beiden Jahrzehnten seines Bestehens als eine Institution mit spezifischen Bedürfnissen. Von einem vornehmlich privaten Raum gelangte es in ein offizielles Gebäude und schließlich als dem botanischen Garten ebenbürtige Sammlung in diesen zurück. Handelte es sich zum Ende des 18. Jahrhunderts noch um einen uneinheitlichen Zusammenschluss von Objekten, kann die Sammlung in der Mitte des 19. Jahrhunderts als etablierte Institution angesehen werden. Während das Herbarium in seinen Anfangsjahren seine Bedürfnisse und Ansprüche grundlegend aushandeln musste, waren bei der Rückkehr ausführliche Argumentationen nicht mehr vonnöten. Wie selbstverständlich wurde es als Sammlung wieder im botanischen Garten integriert. Das verdeutlicht nicht zuletzt die Tatsache, dass das Herbarium ohne weitere Verhandlungen mit dem Gartenmeister in die bis dahin von ihm bezogene Wohnung untergebracht wurde, während dieser selbst ausziehen und auf andere Räumlichkeiten ausweichen musste. ${ }^{135}$ Auch die Formulierung einer, Instruktion für den Assistenten' des Herbariums zeugt von der Etablierung der Sammlung. ${ }^{136}$ Die Mitarbeit eines Gehilfen ist zwar bereits für das Jahr 1833 belegt, wurde 1843 offiziell vom Kuratorium genehmigt und die Bearbeitung damit schon früh in Teilen an diesen abgegeben. ${ }^{137}$ Doch musste Bartling in seinen den Verzeichnissen über die Einnahmen und Ausgaben beigelegten Berichten die Notwendigkeit einer

134 Vgl. UniA GÖ, Sek., 528, Schreiben Bartlings an den Verwaltungsausschuss der Universität, 24.6.1852. Hintergrund für den Umzug ist der Ausbau des Meisterschen Hauses, dessen Beletage nun ebenfalls zu Auditorien umgebaut werden sollte.

135 Vgl. UniA GÖ, Kur., 5605, Schreiben des Universitätskuratoriums an den Verwaltungsausschuss der Universität Göttingen, 5.4.1852.

136 Vgl. UniA GÖ, Sek., 450.4, Schreiben des Universitätskuratoriums an Bartling, 4.1.1853, Anlage, Instruktion für den Assistenten am königlichen Universitäts-Herbarium.

137 Vgl. UniA GÖ, Kur., 5584, Schreiben Bartlings an das Universitätskuratorium, 4.9.1833; UniA GÖ, Kur., 5592, Schreiben des Universitätskuratoriums an Bartling, 19.8.1843. Der erste Gehilfe im Herbarium war der „Studiosus Schlothauber [sic!]“, bei dem es sich vermutlich um August Friedrich Schlotthauber (1801-1872) handelte. Dieser findet allerdings nur in einem einzigen Schreiben Erwähnung, aus dem hervorgeht, dass dieser sich neben der Bearbeitung des Herbariums besonders der Vervollständigung der, vaterländischen` Flora widmete. Für die hierfür erforderlichen Exkursionen erhielt er vom Kuratorium sogar eine finanzielle Unterstützung. Vgl. UniA GÖ, Kur., 5584, Schreiben Bartlings an das Universitätskuratorium, 4.9.1833; Wagenitz 1988, S. 154. Neben dem Assistenten gab es auch Hilfsarbeiter, die in Tagelöhnen bezahlt wurden. So wird im Rechnungsjahr 1836/37 der Schreiber Trautmann für Tätigkeiten im Herbarium bezahlt. Vgl. PWI.Syst.Geobotan.HV1. In den Rechnungsjahren 1847/48 und 1848/49 hilft einer der Gartengehilfen im Herbarium aus. Vgl. PWI.Syst.Geobotan.HV6; PWI.Syst.Geobotan.HV7. Und für das Rechnungsjahr 1851/52 liegen vier Rechnungen für August Bock vor, der Pflanzen aufklebte. Vgl. UniA GÖ, Kur., 5592, Rechnung über Einnahme \& Ausgabe für das Universitäts-Herbarium zu Göttingen vom 1 Juli 1851 bis dahin 1852, 16.11.1852. 
Assistentenstelle immer wieder aufs Neue begründen und erstreiten. ${ }^{138}$ Erst durch ein genaues Abstecken gewann dieser Tätigkeitsbereich auch längerfristig an Legitimation. ${ }^{139}$

Einen solchen Weg konnte die Sammlung nur deshalb gehen, weil ihren Objekten zunehmend Wert zugesprochen wurde. Als zentralem Bestandteil botanischer Forschung wurde sowohl den einzelnen Herbarbelegen als auch der Sammlung als Ganzes mit der Gründung des Herbariums eine erhöhte Wertigkeit beigemessen. Kriterien für ihre wissenschaftliche Bedeutsamkeit waren dabei Vollständigkeit und Nützlichkeit, ihre systematische Sortierung, die selektive Auswahl der aufzunehmenden Objekte sowie eine Genauigkeit in deren Bestimmung und Verzeichnung. Um diesen Ansprüchen und Anforderungen zu entsprechen und sich diesen auch zu verpflichten, entstanden genannte Richtlinien. Es wurden Qualitätsmaßstäbe formuliert, die sich auf das Zurichten der einzelnen Präparate sowie auf die Art und Weise der Unterbringung der Sammlung auswirkten. Und nur durch die offizielle Anerkennung und Wertzuschreibung konnte sich die Institution schließlich auch räumlich etablieren und ausgebaut werden. Die Instruktionen und Regularien für das Göttinger Herbarium, mit denen dessen Wertigkeit und damit auch die Sammlung als solche abgesichert werden sollten, stammten dabei zwar aus Bartlings Feder, entstanden aber ganz deutlich auch vor dem Hintergrund damaliger Sammlungsdynamiken und ganz konkret in Anlehnung an vergleichbare Institutionen. Die Herausbildung des Göttinger Herbariums illustriert beispielhaft die Entwicklung von der Wertschätzung des Kuriosen und Repräsentativen hin zu einer nach wissenschaftlichen Prinzipien geordneten Sammlung. Dem Konkurrenzprinzip der Georgia Augusta folgend, wurden sodann in Bartlings Plädoyers für die Einrichtung eines Herbariums immer wieder andere Sammlungen vergleichend herangezogen. Explizit bezieht sich Bartling beispielsweise in einem Schreiben an das Kuratorium von 1851, in dem er sich sehr ausführlich über die Bedeutsamkeit

138 Vgl. UniA GÖ, Kur., 5592, Schreiben Bartlings an das Universitätskuratorium, 15.2.1847; UniA GÖ, Kur., 5592, Schreiben Bartlings an das Universitätskuratorium, 21.3.1851; UniA GÖ, Kur., 5603, Schreiben Bartlings an das Kabinett Seiner Majestät des Königs, 25.10.1847.

${ }^{139}$ Die Instruktion regelte die Arbeitszeiten des Assistenten sowie seine Aufgaben im Herbarium. Dazu gehörten das Einordnen der in die Sammlung aufzunehmenden Objekte und die Führung des Katalogs, die Konservierung der Sammlung (zum Beispiel das regelmäßige Durchsehen der Pflanzenpakete zum Schutz gegen Insekten), die ordnungsgemäße Aufstellung und äußerliche Bezeichnung der Pflanzenpakete und anderer Gegenstände, die Aufbewahrung der ungeordneten Vorräte und der zum Tausch bestimmten Dubletten sowie die Instandhaltung der Ausstattung. Während der Öffnungszeiten der Sammlung war der Assistent für das Heraussuchen und Einordnen der zur Ansicht vorgelegten Objekte verantwortlich. Dabei hatte er sicherzustellen, dass diese nicht zu Schaden kamen. Auch hatte er ein Verzeichnis über die entliehenen Pflanzen zu führen. Vgl. UniA GÖ, Sek., 450.4, Schreiben des Universitätskuratoriums an Bartling, 4.1.1853, Anlage, Instruktion für den Assistenten am königlichen Universitäts-Herbarium. 
und den Nutzen der Sammlung äußert, auf das Münchner Herbarium. ${ }^{140}$ Zur Bekräftigung seiner Position legte Bartling seinem Schreiben sogar einen von Carl Friedrich Philipp von Martius (1794-1868) verfassten und ihm zugesandten Aufsatz über das Münchner Herbarium bei. ${ }^{141}$ Dabei unterließ er nicht die Bemerkung, dass es sich dabei um einen der ausgezeichnetsten Botaniker handelte und der Bericht auch für die Göttinger botanischen Sammlungen „[...] mehrfaches Interesse darbieten dürfte $[\ldots]^{\text {“142 }}$. Aus dem Aufsatz tritt deutlich hervor, dass viele Prinzipien der Göttinger Sammlung auch in dem 1813 gegründeten Münchner Herbarium wirksam gewesen sind. Dieses hatte ebenso mehrere Abteilungen und umfasste neben einem allgemeinen Herbarium auch eine lokale Sammlung. Mit dem ,Herbarium Boicum` machte man sich auch in München die Erforschung des Königreichs zur besonderen Aufgabe. Das Zurichten der Herbarbelege fand in gleicher Weise statt wie in der Göttinger Sammlung. Ebenso wichtig nahm man die der Aufstellung vorausgehende wissenschaftliche Untersuchung und systematische Bestimmung des Pflanzenmaterials. Und auch in München mussten der Fonds sowie das Verhältnis von Herbarium und botanischem Garten ausgehandelt werden. Einen Gehilfen sah man dort genauso wie in Göttingen als dringendes Erfordernis an. Vordergründiges Ziel war in beiden Sammlungen die Repräsentation des gesamten Pflanzenreichs - und das in systematischer Einheitlichkeit. ${ }^{143}$

Dem Herrichten der Sammlung sowie deren Vergrößerung und Vervollständigung wurde dabei in Göttingen eine solche Aufmerksamkeit gewidmet, dass von Nutzung und Zugänglichkeit zunächst kaum eine Rede sein konnte. Bis zu diesem Punkt mussten offenbar tatsächlich einige Jahre verstreichen. In seinem Bericht über den botanischen Garten, den Bartling 1837 verfasste - im selben Jahr also, in dem er ordentlicher Professor und Direktor wurde und die Georgia Augusta ihr 100-jähriges Bestehen feierte - benannte er das Herbarium als Institution an keiner Stelle. ${ }^{144}$ Er merkte an, dass seit der letzten Abhandlung über den

\footnotetext{
140 Vgl. UniA GÖ, Kur., 5592, Schreiben Bartlings an das Universitätskuratorium, 21.3.1851. Das Münchner Herbarium wird heute auf Platz 18 der 100 größten Herbarien der Welt gelistet. Vgl. Thiers 2019, S. 7. Als eines der ersten institutionalisierten Herbarien Deutschlands galt Bartling die Münchner Sammlung vermutlich auch deshalb als Vorbild, da er sich während mindestens zwei Besuchen im Rahmen von Forschungsreisen mit ihr vertraut gemacht hatte. Vgl. Stafleu 1987, S. 165; UniA GÖ, Kur., 5584, Schreiben Bartlings an das Universitätskuratorium, 8.12.1830; UniA GÖ, Kur., 5584, Schreiben Bartlings an das Universitätskuratorium, 23.10.1838.

141 Vgl. UniA GÖ, Kur., 5584, Das Königliche Herbarium zu München, 1850, Separatabdruck aus den Münchner Gelehrten Anzeigen Bd. 31, Nr. 89-93. Der Aufsatz wurde von dem zugehörigen Schreiben getrennt und in eine andere Akte einsortiert.

142 UniA Gö, Kur., 5592, Schreiben Bartlings an das Universitätskuratorium, 21.3.1851.

143 Dass die systematische Einheitlichkeit allerdings häufig nur eingeschränkt zur Umsetzung gebracht wurde, zeigt sich am Beispiel der Separatsammlungen sowie generell an der Aufgliederung der Sammlung in unterschiedliche Abteilungen.

144 Vgl. Bartling 1837.
} 
botanischen Garten, die 1793 von dem damaligen Professor Hoffmann verfasst worden war, das Institut an Umfang und Inhalt an Bedeutung wesentlich zugenommen habe. ${ }^{145}$ Auch wurde die Königliche Universitäts-Bibliothek als wichtiges fachliches Hilfsmittel der Botanik aufgeführt, ihr Umfang hervorgehoben sowie der Ankauf des Fischer-Herbariums angemerkt. ${ }^{146}$ Doch das 1832 gegründete und seit 1834 mit eigenen, zweckdienlichen Räumlichkeiten versehene Herbarium fand keine Erwähnung. Das ist umso spannender, da Bartling als Alleinstellungsmerkmal des Gartens seine Zugänglichkeit nannte. Bartling schrieb, ,[...] dass kein anderes Institut der Art zugänglicher ist, und keines leichter, bequemer und vollständiger benutzt werden kann." 147 So böte der botanische Garten nicht nur den Gelehrten und Studierenden vor Ort, sondern auch „, [...] jeder anständigen Person [... $]^{\text {“148 }}$ jeden Tag für einige Stunden Zutritt zu allen Teilen. Während der Garten außerdem im Rahmen der Vorbereitungen zur Säkularfeier verschiedenen Instandsetzungen unterzogen wurde und auf Vorschlag Bartlings eine aktuelle Lithographie des Grundrisses angefertigt wurde, findet das Herbarium in diesem Rahmen keine Erwähnung. ${ }^{149}$ Die Sammlung getrockneter Pflanzen wurde tatsächlich erst ab Ostern 1838 für eine Stunde wöchentlich zur freien Benutzung für Studierende und Botaniker*innen freigegeben. ${ }^{150}$ Dass allerdings auch damit der von Bartling angestrebte und für eine zunehmend an Wert gewinnende Sammlung notwendige Grad der Zugänglichkeit nicht erreicht worden war, macht ein Schreiben von 1848 deutlich:

„Hinsichtlich der Benutzung des Universitäts-Herbariums erlaube ich mir zu bemerken, daß zwar jedem, der dieserhalb sich an mich gewendet hat, die Einsicht desselben stets erstattet worden ist, daß ich jedoch noch nicht im Stande gewesen bin, wöchentlich bestimmte Stunden für die Benutzung der botanischen Sammlungen anzusetzen, weil eine solche jedenfalls nur unter ganz specieller Beaufsichtigung, die meine Anwesenheit erfordern würde, geschehen könnte. Insbesondere erschwert diese Einrichtung der Umstand, daß das Local, in welchem das Universitäts-Herbarium aufbewahrt wird, sich nicht in dem botanischen Garten selbst oder doch in dessen unmittelbarer Nähe befindet, ein Umstand, durch welchen überhaupt die umfassendere Benutzung jener an Umfang und wissenschaftlichem Werthe allmälig bedeutend gewordenen Sammlung, namentlich für den botanischen Garten und für die Vorlesungen vielfältig beeinträchtigt und in manchen Fällen fast unmöglich gemacht wird.“151

\footnotetext{
145 Vgl. ebd., S. 3.

146 Vgl. ebd., S. 8.

147 Ebd.

148 Ebd.

${ }^{149}$ Vgl. UniA GÖ, Kur., 5588, Schreiben Bartlings an das Universitätskuratorium, 24.5.1837.

150 Vgl. UniA GÖ, Kur., 5592, Schreiben Bartlings an das Universitätskuratorium, 17.2.1838.

151 UniA GÖ, Kur., 5592, Schreiben Bartlings an das Universitätskuratorium, 14.11.1848.
} 
Bis botanischer Garten und Herbarium als Institutionen vergleichbare Bedeutsamkeit besaßen, waren also vielschichtige Aushandlungsprozesse notwendig. Spezialisierung und Standardisierung sind dabei Ausdruck von zunehmender Wertzuschreibung und dem Bedürfnis nach Absicherung und Erhaltung dieser Wertigkeit. Was 1830 in den ersten Schreiben Bartlings zur Gründung des Herbariums mit einer Abgrenzung von den Altbeständen und einer Abwertung der Privatsammlung begann, etabliert sich bis $1852 \mathrm{zu}$ einer institutionalisierten Sammlung, die nach damaligem wissenschaftlichem Standard aufgebaut und ausgestattet sein sollte. Sie zeichnete sich durch eine eigene Infrastruktur und ein klar formuliertes Sammlungsprofil aus. 


\subsection{Pflanzenexemplare akquirieren}

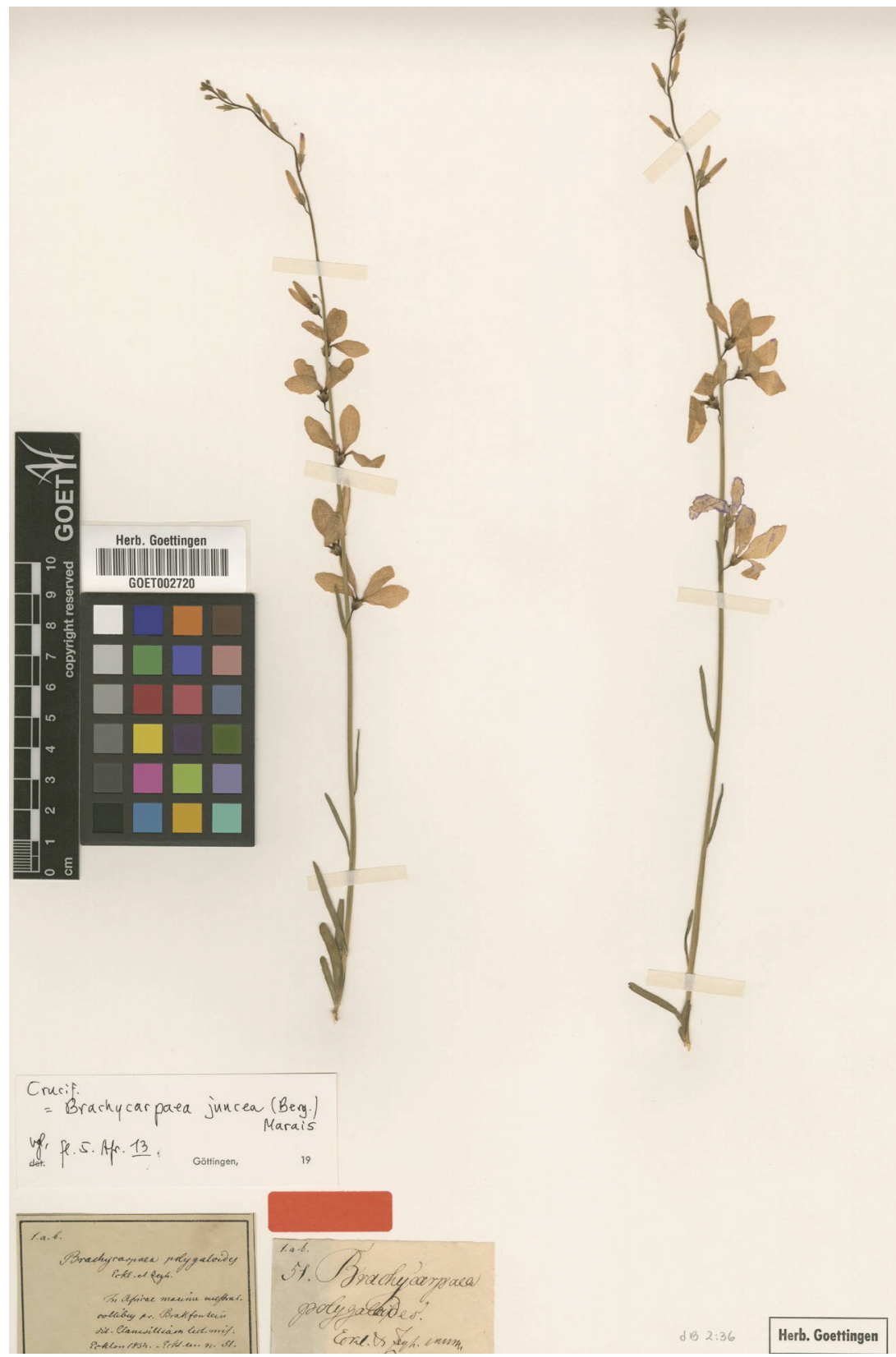

Abb. 8: Herbarbeleg von Brachycarpea juncea Marais, gesammelt von C. F. Ecklon im September 1834 auf einem Hügel bei ,Brackfontein'(Clanwilliam), Herbarium Göttingen, GOET002720. 
$\mathrm{Zu}$ den von Bartling formulierten Voraussetzungen für die Aufnahme in die Sammlung gehörte, dass jeder Pflanzenbeleg nach gleichem Prinzip aufbereitet wurde - egal ob aus eigener Forschungstätigkeit oder auf anderem Wege erworben. Deshalb weist der Herbarbeleg zu Brachycarpaea polygaloides Eckl. \& Zeyh. (Brachycarpea juncea Marais) auch zwei Etiketten auf. Sie verweisen aber nicht allein auf eine Bearbeitung des Materials durch Bartling, sondern in diesem Fall gleichzeitig auf die Sammler oder den*die Übermittler*in des Pflanzenmaterials. Denn auch wenn nicht mit Sicherheit festgestellt werden kann, aus welcher Hand das rechte der beiden Etiketten stammt, so stellt es doch den letzten Verweis auf den vollzogenen Besitzwechsel des Präparats dar: Dieses stammt aus der Sammlung von Christian Friedrich Ecklon (1795-1868) und Carl Ludwig Philipp Zeyher (1799-1858) und wurde, so informiert das umfangreichere linke Etikett, 1834 übermittelt. Diese knappe Jahresangabe täuscht jedoch über den Stellenwert hinweg, den die Akquirierung dieses Konvoluts darstellte. Der Beleg ist Teil eines umfangreichen Ankaufs, der das Herbarium über mehrere Jahre hinweg finanziell belastete.

Das Exemplar von Brachycarpaea polygaloides kann stellvertretend für eine Vielzahl erstmals beschriebener Arten der Kap-Flora stehen. ${ }^{152}$ Die Nummer 51 sowie die Abkürzung ,enum.', beziehungsweise ,en.' auf dem Göttinger Etikett, verweisen auf die Originalbeschreibung in Enumeratio plantarum Africae australis extratropicae. ${ }^{153}$ Diese dreibändige Veröffentlichung zu den gesammelten Pflanzen erschien in den Jahren 1835, 1836 und 1837 und ist als Begleitpublikation zu den Belegen zu verstehen, die Ecklon und Zeyher einzeln aber auch gemeinsam von 1829 bis 1832 in der Kapregion sammelten. ${ }^{154}$ Über die Ergebnisse der Reise und den bevorstehenden Verkauf von Pflanzenexemplaren informierte Ecklon selbst 1833 in einem Bericht, der in der botanischen Zeitschrift Linnaea erschien. ${ }^{155} \mathrm{Als}$ Vorsteher der Göttinger Sammlung schien Bartling auf diesen reagiert, die Chance genutzt und einen Teil der angebotenen Dubletten trotz Schuldenaufnahme für die Universitätssammlung akquiriert zu haben.

\footnotetext{
152 Vgl. Wörz 2016, S. 80.

153 Vgl. Ecklon \& Zeyher 1835, S. 7.

154 Vgl. Wörz 2016, S. 77-79.

155 Vgl. Ecklon 1833.
} 


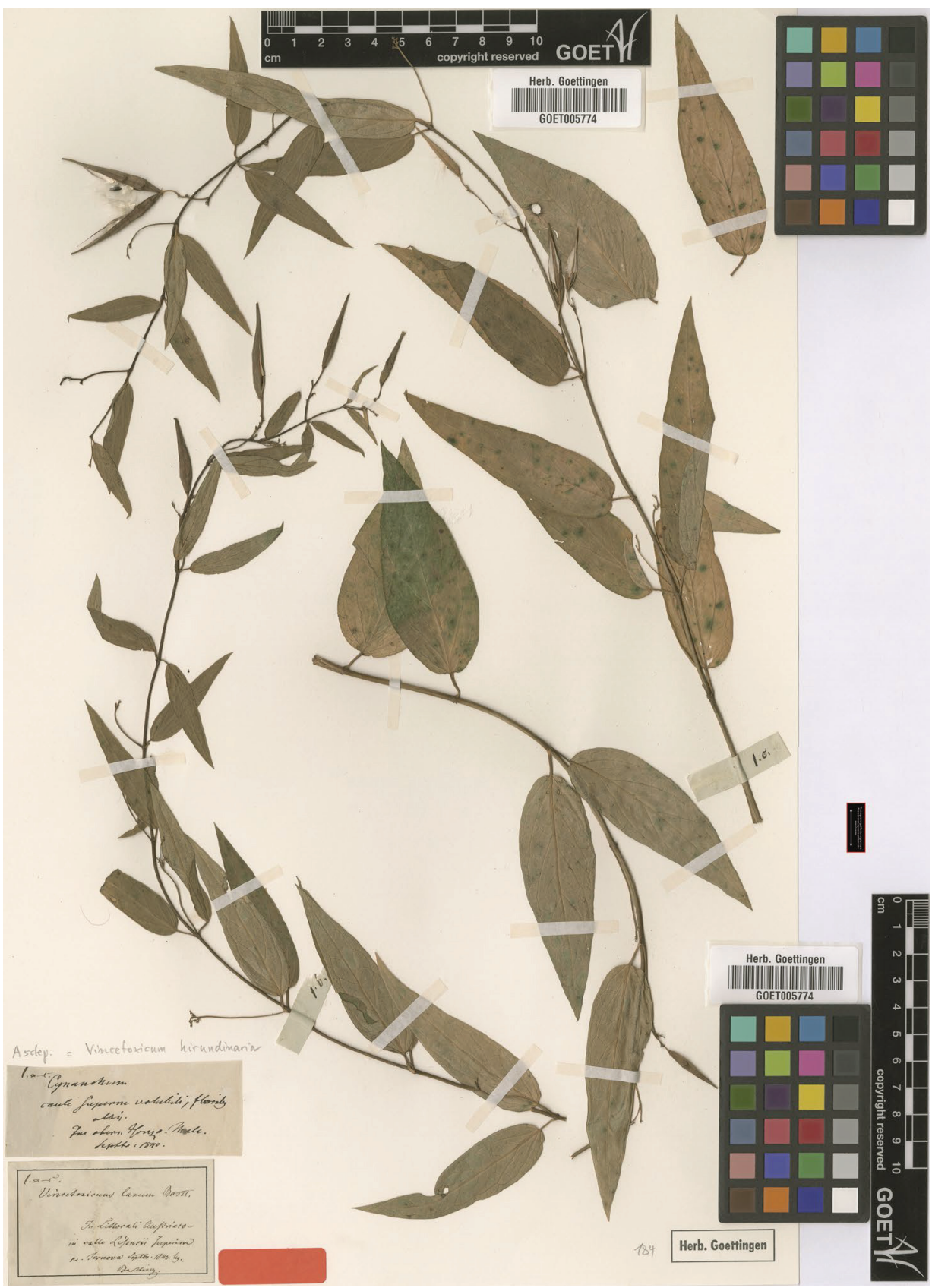

Abb. 9: Herbarbeleg von Vincetoxicum birundinaria Medik., gesammelt von F. G. Bartling im September 1840 im oberen Isonzo-Tal bei Ternova (Ternova d'Isonzo/Trnova ob Soči), Herbarium Göttingen, GOET005774. 


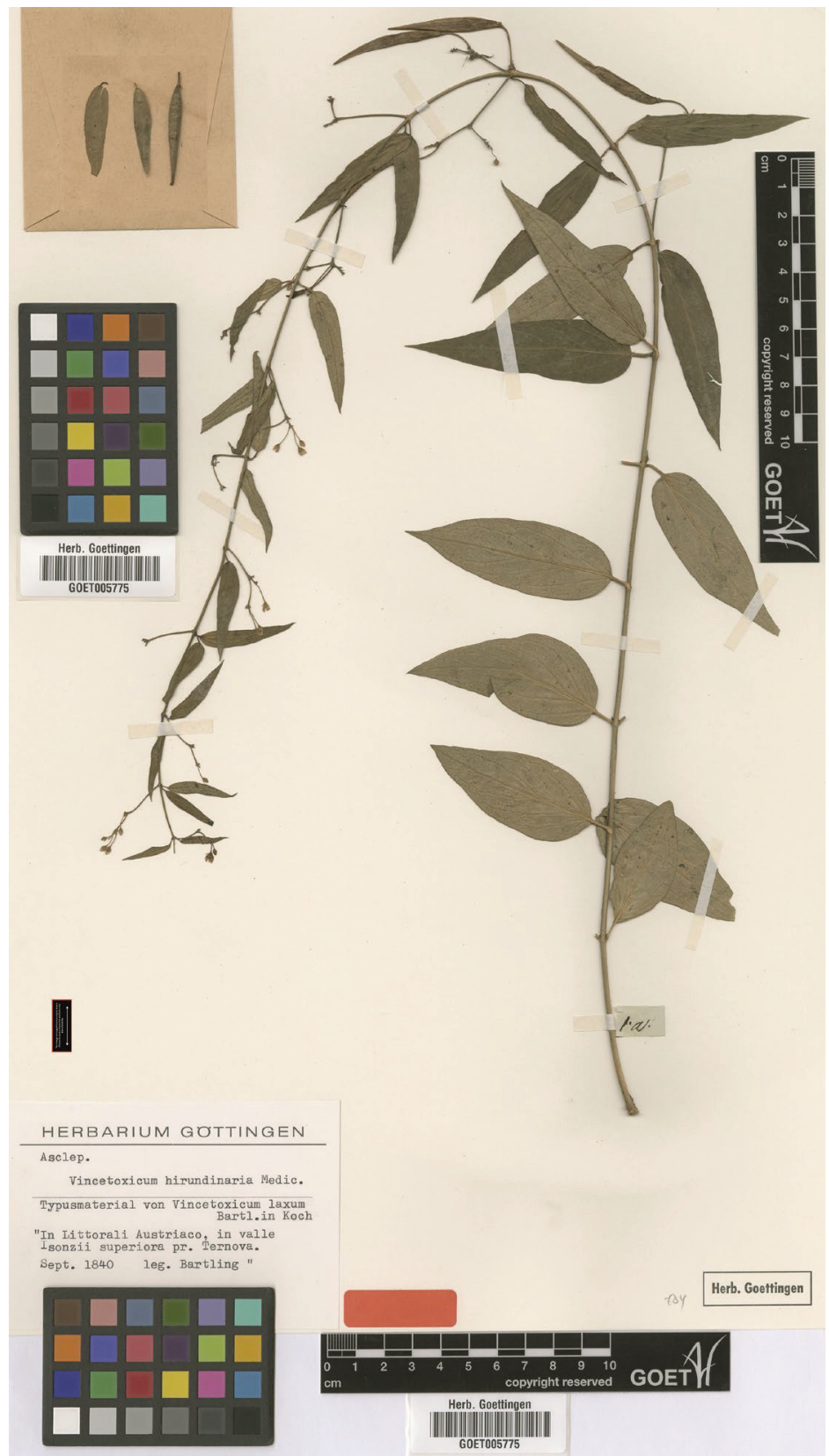

Abb. 10: Herbarbeleg von Vincetoxicum hirundinaria Medik., gesammelt von F. G. Bartling im September $1840 \mathrm{im}$ oberen Isonzo-Tal bei Ternova (Ternova d'Isonzo/Trnova ob Soči), Herbarium Göttingen, GOET005775. 
Auch der Beleg zu Vincetoxicum laxum Bartl. (Vincetoxicum birundinaria Medik. 150) verfügt über zwei Etiketten. Diese stehen hier allerdings nicht für einen Eigentumswechsel, sondern für den Arbeitsvorgang von einer eher flüchtigen Bezeichnung hin zur Beschriftung nach Maßgabe der Universitätssammlung. Denn während die von Ecklon und Zeyher stammende Pflanze angekauft worden ist, wurde dieser Beleg von Bartling auf einer Forschungsreise gesammelt. Die Angabe zum Sammeldatum ,September 1840' sowie zum Sammelort ,In Littorali Austriaco - in valle Lisoncii superiore pr. Ternova ${ }^{6} 157$ lassen ihn zweifelsfrei der zweimonatigen, vom 2. September bis zum 30. Oktober unternommenen Exkursion zuordnen. Sie führte Bartling gemeinsam mit zwei Kollegen von Bad Reichenhall bei Salzburg bis nach Fiume ${ }^{158}$ durch das Kaisertum Österreich. ${ }^{159}$ Finanziert durch das Kuratorium trug Bartling hierbei verschiedene Naturalien für die Sammlungen der Universität zusammen. ${ }^{160}$ Dazu gehörten auch 250 Pflanzenarten in 900 Exemplaren, die von ihm gesammelt und getrocknet wurden und dem Herbarium zugutekamen. Zu diesem Teil der ,Ausbeute' ist Vincetoxicum laxum zu zählen. Während die erste der Beschriftungen vermutlich noch auf der Reise oder im Rahmen der ersten Durchsicht der Naturalien nach der Rückkehr erstellt wurde, entspricht das zweite Etikett den üblichen an die Sammlungsobjekte gestellten Anforderungen. Genauso wie bei Brachycarpaea polygaloides unterscheiden sich die beiden Etiketten also in ihrem Informationsgehalt. Während die von Ecklon und Zeyher stammende Pflanze auf ihrem Schild aber insgesamt weniger Informationen trägt, liegt bei den Etiketten der von Bartling gesammelten Pflanze die Differenz im Informationsgehalt selbst: Das jüngere Etikett hält hier nicht nur konkretere Angaben zum Ort und Sammler bereit. Vielmehr entfallen die Angaben zu Blütenfarbe und den Eigenschaften der Pflanzenstängel der ursprünglichen Beschriftung und werden durch den genauen wissenschaftlichen Namen ersetzt. Anders als das Etikett zu Brachycarpaea polygaloides verweist die ältere Beschriftung des Belegs von Bartling also auf die mit der Sammeltätigkeit verbundenen Arbeitsschritte und nicht auf eine Verkaufsvorbereitung.

\footnotetext{
${ }^{156}$ Ein synonym genutzter Name ist außerdem Vincetoxicum laxum (Bartl.) K. Koch.

${ }^{157}$ Die Pflanze wurde im oberen Isonzo-Tal nahe bei Ternova gesammelt. Ternova d'Isonzo (Trnova ob Soči) gehört heute zur Republik Slowenien.

158 Fiume ist der italienische Name der Stadt Rijeka im heutigen Kroatien.

159 Vgl. UniA GÖ, Kur., 5584, Bericht über die von dem Professor Bartling in den Monaten September und Oktober 1840 durch einen Teil der österreichischen Monarchie gemachten naturwissenschaftlichen Reise, o. D.

160 Vgl. UniA GÖ, Kur., 5584, Bericht über die von dem Professor Bartling in den Monaten September und Oktober 1840 durch einen Teil der österreichischen Monarchie gemachten naturwissenschaftlichen Reise, o. D., Anlage A, Verzeichnis der Gegenstände, welche der Unterzeichnete auf der im September und Oktober gemachten Reise für die Universität akquiriert hat.
} 
Mit seiner Gründung 1832 und der damit einhergehenden Formulierung genauer Richtlinien besaß das Göttinger Herbarium einen expliziten Sammelauftrag. Die Bestimmung der neu institutionalisierten Universitätssammlung war die Bereitstellung „[...] ausgewählter Exemplare einer möglichst großen Anzahl von

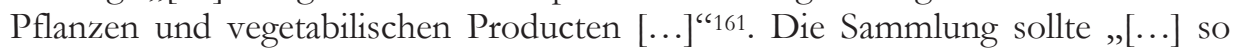
viel es die Umstände und die Mittel erlauben, zweckmäßig vermehrt [...]"162 werden. Doch auf welche Weise gelangten Pflanzenexemplare in das Herbarium? Welche Objekte wurden als wichtig für die Sammlung wahrgenommen? Nach welchen Regeln und Kriterien fand der Erwerb statt? Und wo traten Probleme oder besondere Herausforderungen auf?

Die Überlieferung zu den Prozessen der Akquirierung von Pflanzenmaterial im Göttinger Herbarium in der Zeit von 1832 bis 1852 ist lückenhaft. Der von Bartling und seinem Gehilfen geführte Katalog ist heute nicht mehr erhalten. Ein Überblick über die Art und Weise der Aufnahme von Sammlungen in das Herbarium kann deshalb größtenteils nur über eine Zusammenschau anderer Quellen gewonnen werden. Hierzu gehört in erster Linie der jährliche Schriftverkehr zwischen Bartling und dem Universitätskuratorium bezüglich der Einnahmen und Ausgaben des Herbariums. ${ }^{163}$ Denn auch wenn die von Bartling erstellten und als Register angelegten Rechnungen nur für zehn Rechnungsjahre erhalten sind, so geben die dazu verfassten Bemerkungen des Kuratoriums sowie die den Rechnungen beigefügten Berichte doch Aufschluss über den Zuwachs der Sammlung. ${ }^{164}$ In letzteren gibt Bartling ausführlich Auskunft über den Bestand des Herbariums und das hinzugekommene Pflanzenmaterial. Darüber hinaus wurde ab 1878 von dem Privatdozenten und Assistenten am Herbarium Oscar Drude (1852-1933) ein Akzessionskatalog begonnen, der Nachträge für die vorausgegangenen Jahre beinhaltet. ${ }^{165} \mathrm{Da}$ die Notizbücher Bartlings

161 UniA GÖ, Kur., 5584, Schreiben Bartlings an das Universitätskuratorium, 14.11.1832, Anlage A.

162 UniA GÖ, Kur., 5584, Schreiben Bartlings an das Universitätskuratorium, 14.11.1832, Anlage A, C, Von der Verwaltung des Universitäts-Herbarii.

163 Die Abrechnungen des Herbariums und des botanischen Gartens erfolgten getrennt voneinander, da jede Institution einen eigenen Fonds zur Verfügung gestellt bekam. Vgl. UniA GÖ, Kur., 5560; UniA GÖ, Kur., 5592.

164 Rechnungen sind ausschließlich für die Rechnungsjahre 1836/37, 1837/38, 1839/40, 1840/41, 1843/44, 1847/48, 1848/49, 1851/52, 1854/55 sowie 1860/61 erhalten.

Vgl. PWI.Syst.Geobotan.HV1; PWI.Syst.Geobotan.HV2; PWI.Syst.Geobotan.HV3;

PWI.Syst.Geobotan.HV4; PWI.Syst.Geobotan.HV5; PWI.Syst.Geobotan.HV6; PWI.Syst.Geobotan.HV7; PWI.Syst.Geobotan.HV8; PWI.Syst.Geobotan.HV9; UniA Gö, Kur., 5592, Rechnung über Einnahme \& Ausgabe für das Universitäts-Herbarium zu Göttingen vom 1. Juli 1851 bis dahin 1852. Dass ursprünglich zu jedem Rechnungsjahr ein Register vorgelegen haben muss, ist der ,Instruction für die Rechnungs-Führung wegen des Universitäts-Herbarii zu Göttingen“ vom 4. Dezember 1832 zu entnehmen, die allen erhaltenen Rechnungsaufstellungen Bartlings vorgeschaltet ist.

165 Vgl. PWI.Syst.Geobotan.HE2; Wagenitz 1982, S. 44. 
nicht überliefert und auch die Korrespondenzen nur in Bruchstücken erhalten sind, besitzen außerdem die von ihm nach den Exkursionen abgefassten und an das Kuratorium gesandten Reiseberichte besonders hohe Relevanz.

Wie bereits herausgestellt worden ist, wurde der Sammlung getrockneter Pflanzen mit ihrer Institutionalisierung eine neue Wertigkeit zugesprochen. Diese spiegelte sich in der Zurichtung der Objekte, in ihrer Sortierung und räumlichen Aufbewahrung wider, denn wertvoll war ein Pflanzenbeleg, der genau beschriftet und bestimmt worden war und nach Verrichten dieser Schritte in ein bestehendes System eingeordnet und damit nutzbar gemacht wurde. Die Sammlung als Ganzes verfolgte dabei ein enzyklopädisches Sammlungsideal, das sich vor allem durch den Wunsch nach Vollständigkeit und Komplettierung auszeichnete. ${ }^{166}$ Und auch auf die Frage, wie diesem Wunsch nachzukommen sei, geben die von Bartling verfassten Instruktionen zum Herbarium Auskunft: Drei der zentralen Sammelstrategien, der Ankauf, die Schenkung und der Tausch, werden in ihnen explizit benannt. ${ }^{167}$ Hinzu kamen die in kleinerem oder größerem Umfang durchgeführten Exkursionen ins In- und Ausland, auf denen ebenfalls neues Material zusammengetragen wurde. Alle vier Sammelstrategien waren in der Zeit von 1832 bis 1852 wirksam und trugen ihren Anteil zur Erweiterung der Sammlung bei. ${ }^{168}$ Wie häufig die einzelnen Möglichkeiten der Pflanzenakquisition zum Einsatz kamen und in welchem Verhältnis sie genau zueinander standen, kann für das Göttinger Herbarium aber nur eingeschränkt bestimmt werden. Das erklärt nicht nur das heutige Fehlen des ursprünglichen Katalogs, sondern auch, dass nicht immer vermerkt worden ist, auf welche Weise die Konvolute in die Sammlung gelangt sind. Da hauptsächlich der Schriftverkehr zu den Rechnungen über die Einnahmen und Ausgaben des Herbariums als Quelle zur Verfügung steht, ist außerdem zu vermuten, dass viele der Pflanzen, die im Tauschgeschäft oder als Geschenk erlangt worden sind, dort nur dann erwähnt wurden, sofern sie sehr bedeutsam für die Sammlung waren.

\footnotetext{
166 Vgl. Macho 2000, S. 66-68.

167 Vgl. UniA GÖ, Kur., 5584, Schreiben Bartlings an das Universitätskuratorium, 14.11.1832, Anlage A, C, Von der Verwaltung des Universitäts-Herbarii.

168 Damit unterschieden sich die möglichen Wege der Akquisition im Herbarium zunächst nicht von jenen in den naturhistorischen Museen des 19. Jahrhunderts, wie sie der Historiker Carsten Kretschmann in seiner Monografie für vier Häuser exemplarisch dargelegt hat. Auch Kretschmann unterscheidet zwischen diesen vier Sammelstrategien. Im Mittelpunkt seiner Studie stehen das Großherzogliche Naturalienkabinett in Karlsruhe, das Museum für Naturkunde in Berlin, das Senckenberg-Museum in Frankfurt am Main und das Städtische Museum für Natur-, Völker- und Handelskunde in Bremen. Die vier Häuser wurden nicht nur ausgewählt, da sie im 19. Jahrhundert zu den wichtigsten Einrichtungen dieser Art gehörten, sondern auch, weil sie unterschiedliche Typen des Museums vertreten: die höfische Sammlung (Karlsruhe), das Universitätsmuseum (Berlin) sowie das bürgerliche Vereinsmuseum in zwei unterschiedlichen sozialen Kontexten (Bremen, Frankfurt). Vgl. Kretschmann 2006, S. 139-184.
} 


\section{Ankauf}

Es kann deshalb nur unter Vorbehalt behauptet werden, dass die meisten Pflanzen als Ankäufe in die Sammlung gelangten. Hinsichtlich der Käufe ist allerdings von einem weitestgehend vollständigen Bild auszugehen. Denn dem Herbarium stand als dem Königlichen Universitätskuratorium unterstellte Institution ein jährlicher Fonds zu, von dem auch die Anschaffung von Pflanzenmaterial bestritten werden sollte. Durch die Verbindung nach Hannover war die Sammlung also durch einen regelmäßigen Etat abgesichert, gleichzeitig hatte sie aber auch eine nicht zu unterschätzende Abhängigkeit zur Folge: In Bezug auf die Einnahmen und Ausgaben des Herbariums mussten detaillierte Register angefertigt werden, welche gemeinsam mit den Belegen sowie einem Bericht des Vorstehers zur Abnahme und Revision nach Hannover geschickt wurden. Dadurch sind die Ankäufe für das Herbarium heute zwar gut nachzuvollziehen und zu belegen, es ist jedoch davon auszugehen, dass der Rechtfertigungszwang gegenüber dem Kuratorium und besonders die festgelegten Amtswege die Flexibilität in den Prozessen der Akquirierung verminderten. Wie aus den Berichten Bartlings, die den Rechnungen beizulegen waren, deutlich hervorgeht, reichte der vom Kuratorium zur Verfügung gestellte Fonds von jährlich 100 Talern häufig nicht aus. ${ }^{169}$ In der Praxis konnte folglich nur eingeschränkt von einer autarken Verwendung des Etats die Rede sein. $\mathrm{Ob}$ und inwiefern spontane Kaufangebote wahrgenommen werden konnten, bleibt zu hinterfragen. Diejenigen Schreiben, die Ankäufe für das Herbarium dokumentieren, lassen jedoch darauf schließen, dass diese vornehmlich in Vorausplanung und wohlkalkuliert stattfanden. Denn obwohl offensichtlich nicht jeder Ankauf vom Kuratorium genehmigt werden musste, machte der knapp bemessene Fonds es immer wieder notwendig, außerordentliche Zahlungen und Zuschüsse zu beantragen. Umfassende Quellen, die den Ablauf eines Ankaufs für das Herbarium im ,Normalfall' dokumentieren, liegen nicht mehr vor. Dagegen lassen Sonderfälle, Abweichungen und Herausforderungen in den käuflichen Akquirierungen Rückschlüsse auf die Kaufpraxis am Herbarium zu. So ist davon auszugehen, dass für alle Ankäufe, die sich im Rahmen des zur Verfügung gestellten Fonds bewegten, kein Einverständnis eingeholt werden musste. Anders sah dies aus, wenn der Etat überschritten zu werden drohte: Beim Ankauf des Herbariums des Kassler Naturforschers Rudolph Amandus Philippi (1808-1904) im Jahr 1846 musste das Kuratorium aushelfen. ${ }^{170}$ Der Betrag von 150 Talern konnte durch den Fonds allein nicht bestritten werden, sodass Bartling im Voraus in einem Schreiben an das Kuratorium für einen außerordentlichen Betrag von 100 Talern argumentierte. ${ }^{171}$ Die restlichen 50 Taler sollten wie üblich durch den Fonds des Herbariums getragen werden. Tatsächlich lag der durchschnittliche

\footnotetext{
169 Vgl. UniA GÖ, Kur., 5592, Schreiben Bartlings an das Universitätskuratorium, 15.2.1847.

170 Vgl. Wagenitz 1982, S. 126.

171 Vgl. UniA GÖ Kur., 5600, Schreiben Bartlings an das Universitätskuratorium, 10.1.1846.
} 
Preis für einen Ankauf bei ungefähr zehn Talern und es wurden nur selten mehr als 20 Taler pro Sammlung verausgabt. Von den ungefähr 20 deutlich als Kauf zu benennenden Akquirierungen, bei denen außerdem auch der Preis angegeben ist, können demnach insgesamt vier uneingeschränkt als außerordentliche, die Grenzen des Fonds über-schreitende Ankäufe eingestuft werden. Da die Sammlung Philippi, wie aus Bartlings Schreiben hervorgeht, bereits zu einem früheren Zeitpunkt zum Kauf angeboten worden war, ist ein Vorausplanen in diesem Fall möglich gewesen. Ebenso vorausschauend wurde der Ankauf von Bartlings Herbarium geplant. ${ }^{172}$ Der Erwerb der Sammlung südafrikanischer Gewächse des Pflanzensammlers Christian Friedrich Ecklon ist aber als besondere Ausnahme zu werten. Sie gelangte in zwei Sendungen in das Göttinger Herbarium. Sowohl im Rechnungsjahr 1834/35 als auch im Rechnungsjahr 1835/36 schickte dieser Pflanzen aus Hamburg zu, wo er sein gesammeltes Material zu diesem Zeitpunkt bearbeitete. ${ }^{173}$ Laut den den Rechnungen beigelegten Berichten Bartlings wurde die aus diesen Sendungen entstandene Schuld allerdings nicht direkt, sondern erst in den Rechnungsjahren 1836/37, 1840/41 und 1844/45 abbezahlt. ${ }^{174}$ Denn die insgesamt zu verausgabende Summe umfasste etwa 200 Taler und entsprach damit dem doppelten Jahresfonds des Herbariums. ${ }^{175}$ Anders als mit Raten war die Zahlung dieses Betrags offensichtlich nicht umzusetzen. Eine solche Praxis des Ankaufs für den hier betrachteten Zeitraum bleibt jedoch ein Einzelfall. Üblicherweise erfolgte die Zahlung im gleichen Rechnungsjahr, in dem auch die Akquirierung stattgefunden hatte.

Inwieweit der 1843 getätigte Ankauf einer Sammlung von Pflanzen aus dem westlichen Australien im Voraus durchdacht worden war, kann nicht mit Sicherheit angegeben werden. Allerdings konnte sie nur durch einen außerordentlichen Zuschuss des Kuratoriums von 100 Talern erworben werden. Ob dieser aber explizit für den Kauf dieser Sammlung gewünscht war, lässt sich nicht feststellen. Anders als bei den üblichen Ankäufen, die in seinen Schreiben meist lediglich in Verbindung mit Sammelort und Sammler aufgelistet werden, legt Bartling in Bezug auf die Pflanzen Johann August Ludwig Preiss' (1811-1883) allerdings eine genaue Begründung für die Bedeutsamkeit der Akquirierung dar.

\footnotetext{
172 Bartlings Herbarium wurde 1841 für insgesamt 300 Taler angekauft. Vgl. UniA GÖ, Kur., 5584, Kaufvertrag zwischen Bartling und dem Geheimen Justizrat Bergmann, 23.8.1841.

173 Vgl. UniA GÖ, Kur., 5592, Schreiben Bartlings an das Universitätskuratorium, 15.9.1935; UniA GÖ, Kur., 5592, Schreiben Bartlings an das Universitätskuratorium, 19.12.1836.

${ }^{174}$ Die Abwicklung der Zahlungen fand in den Rechnungsjahren 1840/41 und 1844/45 auch nicht mehr über Ecklon selbst statt, der sich zu dieser Zeit bereits wieder in Kapstadt aufhielt, sondern wurde durch den Bevollmächtigten Apotheker Thorey und Johann Georg Christian Lehmann (1792-1860) in Hamburg geregelt. Vgl. PWI.Syst.Geobotan.HV4; UniA GÖ, Kur., 5592, Schreiben Bartlings an das Universitätskuratorium, 6.8.1846; Wörz 2016, S. 78 f. 175 Vgl. UniA GÖ, Kur., 5592, Schreiben Bartlings an das Universitätskuratorium, 6.8.1846.
} 
Die Sammlung sei wichtig,

„[...] da das Universitäts-Herbarium aus jenen Gegenden, deren Flora zu den reichsten und merkwürdigsten gehört, noch gar nichts besaß, da schwerlich sich bald wieder die Gelegenheit zu einer so interessanten Acquisition darbieten dürfte, und da endlich die vom Dr. Preiss im westlichen Neuholland gesammelten Pflanzen in einem besondern zum Theil schon erschienenem Werk = Plantae Preissieanae, für welches auch der ehrerbietigst Unterzeichnete mehrere Familien bearbeitet hat, beschrieben werden, und daher sämmtlich als Original-Exemplare zu betrachten sind.“"176

Durch den Zuschuss des Kuratoriums konnte die Kaufsumme von 115 Talern problemlos bestritten werden, doch macht die Erklärung Bartlings deutlich, dass ein solcher Betrag als ungewöhnlich hoch angesehen wurde. Gleichzeitig illustriert die Aussage beispielhaft die für die Akquirierungen üblicherweise angebrachten Argumente: Seltenheit und Ausgefallenheit der Sammlung galten immer wieder als wichtige Gründe für die Akquisition. Auch die Tatsache, dass es sich um eine besondere Gelegenheit handelte, taucht in den Begründungen für Ankäufe vermehrt auf. In Bezug auf die Sammlung Philippi wurde dieser Punkt noch durch den Hinweis bestärkt, es gebe weitere „Kauflustige“177. Da außerdem die Vollständigkeit der Sammlung angestrebt wurde, betonte Bartling es stets, wenn aus Regionen bisher keine Exemplare ins Herbarium aufgenommen worden waren - gerade, wenn die dortige Flora als äußerst artenreich angesehen wurde. Für das Herbarium besonders wertvoll waren dann natürlich jene Sammlungen, die zahlreiche Original-Exemplare beinhalteten - also Pflanzen, an denen neue Arten erstmals beschrieben wurden. ${ }^{178}$ Auch ein dichtes Vorkommen von Dubletten, die in der Folge als Tauschgegenstände genutzt werden konnten, galt als schlagkräftiges Argument für den Erwerb.

\section{Exkursionen}

Doch auch wenn das Herbarium ein enzyklopädisches Sammlungsideal verfolgte, so besaß es zugleich auch eigene Schwerpunkte, die sich durch selbstständiges Sammeln auf Exkursionen sehr viel gezielter verfolgen ließen:

„Was die Herbeischaffung der in die Sammlung aufzunehmenden Gegenstände betrifft, so wird zwar, wie bisher, vieles durch Ankauf oder gelegentlichen Tausch erworben werden müssen, manches aber, besonders für die vor allem wünschenswerthe Vervollständigung der europäischen Flora, auch fernerhin nur durch eigenes Sammeln auf Excursionen und Reisen zu erlangen seyn." 179

\footnotetext{
176 UniA GÖ, Kur., 5592, Schreiben Bartlings an das Universitätskuratorium, 21.6.1845.

177 UniA GÖ, Kur., 5600, Schreiben Bartlings an das Universitätskuratorium, 10.1.1846.

178 Zur besonderen Behandlung der Typenexemplare seit dem späten 19. und frühen 20. Jahrhundert vgl. Daston 2004, S. 158-165.

179 UniA GÖ, Kur., 5592, Schreiben Bartlings an das Universitätskuratorium, 21.3.1851.
} 
Die Pflanzen, die zwischen 1832 und 1852 durch Kauf akquiriert wurden, dienten tatsächlich fast vollständig dem Zuwachs der Hauptsammlung des Herbariums und stammten aus Ländern, die von den Göttinger Botaniker*innen und namentlich von Bartling als Verantwortlicher des Herbariums nicht selbst bereist wurden. ${ }^{180}$ Gerade die ,einheimische', ,vaterländische ${ }^{6}$ Flora, die gemeinsam mit der Hauptsammlung des Herbariums zu seinem vorrangingen Interesse gehörte, wuchs aber durch eigene Exkursionen Bartlings an. Welche Regionen er unter Verwendung dieser Begriffe zu umfassen suchte, ist dabei stark kontextgebunden. Sowohl in Bezug auf die Vervollständigung der zweiten Abteilung im Herbarium, der Flora Hannoverana, als auch im Rahmen von Bartlings Reisen in die Alpen fanden die gleichen Bezeichnungen Anwendung. ${ }^{181}$ Es ist deshalb davon auszugehen, dass mit den Begriffen ,heimisch ${ }^{6}$ und ,vaterländisch ${ }^{6}$ im jeweiligen Zusammenhang sowohl das Königreich Hannover als auch die dem Deutschen Bund zugehörigen Regionen gemeint gewesen sind. Diese Zweiteilung spiegelte sich auch in den von Bartling unternommenen Reisen wider: Im Namen der botanischen Einrichtungen bereiste er zum einen verstärkt die Alpenregionen und ihre Ausläufer. Zum anderen führte er Exkursionen in Niedersachsen, und hier besonders im Harz, sowie Nordhessen durch.

Während die erstgenannten Reisen stets finanziell durch das Kuratorium unterstützt und damit auch genehmigt werden mussten, wurden die Exkursionen innerhalb des Königreichs durch den Fonds des Herbariums sowie durch private Mittel bestritten. ${ }^{182} \mathrm{Da}$ von den in Göttingen zwischen 1832 und 1852 wirkenden Dozierenden keine Notizbücher oder umfangreiche Schriftwechsel vorliegen, können diese Reisen sowie alle privaten Ausflüge und jene Exkursionen, die im Rahmen der Vorlesungen gemeinsam mit den Studierenden unternommen wurden, nicht tiefergehend belegt und nachvollzogen werden. Sie fanden lediglich in den jährlichen Berichten an das Kuratorium kurze Erwähnung. Ob diese Reisen innerhalb des Königreichs also genauso verliefen wie die gut dokumentierten umfangreicheren Reisen, lässt sich deshalb nicht hinreichend beantworten. Aus den Berichten Bartlings geht jedoch hervor, dass sie vor allem der Vervollständigung der einheimischen Flora und damit der zweiten Abteilung des Herbariums zugutekamen. ${ }^{183}$

${ }^{180}$ Darin zeigt sich die Beteiligung auch des Göttinger Herbariums an einer ,Colonial Botany“, die sich, wie Schiebinger und Swan herausstellen, bereits durch den Austausch kolonial geprägter Wissensbestände auszeichnete. Denn auch das ,Weitertragen“ und Kultivieren von Samen und Pflanzen in den „laboratories of colonial botany“ (S. 13) - gemeint sind die botanischen Gärten - werten die Autorinnen als eine solche Partizipation.

Vgl. Schiebinger \& Swan 2005.

181 Vgl. UniA GÖ, Kur., 5592, Schreiben Bartlings an das Universitätskuratorium, 15.2.1847; UniA GÖ, Kur., 5592, Schreiben Bartlings an das Universitätskuratorium, 6.11.1849.

182 Vgl. UniA GÖ, Kur., 5592, Schreiben Bartlings an das Universitätskuratorium, 21.3.1851.

183 Vgl. UniA GÖ, Kur., 5592, Schreiben Bartlings an das Universitätskuratorium, 19.12.1836; UniA GÖ, Kur., 5592, Schreiben Bartlings an das Universitätskuratorium, 14.12.1841; 
Die außerhalb des Königreichs unternommenen Forschungs- und Sammelreisen, die nicht durch den Fonds des Herbariums allein bestritten werden konnten, sind wegen der notwendigen Antragstellung sehr ausführlich dokumentiert. Zu jeder dieser Reisen liegen ein Gesuch Bartlings mit detaillierter Begründung für sein Unternehmen, die Bewilligung des Kuratoriums sowie der nach Beendigung der Reise abgefasste Reisebericht vor. Während die Exkursionen im Inland allein schon aus finanzieller Sicht sowie aufgrund der zurückzulegenden Strecken spontaner durchgeführt werden konnten, war für Reisen außerhalb des Königreichs eine größere Vorausplanung notwendig. 184 Die an das Kuratorium gerichteten Anträge auf Bewilligung des Urlaubes zu einer Forschungsreise waren im Falle Bartlings in ihrem Informationsgehalt auch sehr umfangreich. Genau formulierte Bartling sein Forschungsinteresse und die mit der Reise verbundenen Absichten und Ziele. Auch enthielten die Anträge das Reiseziel sowie Angaben zum Reiseverlauf und den erwarteten Reisekosten. Mit Blick auf den Adressaten gaben die Schreiben darüber hinaus immer auch Antwort auf die Frage nach der konkreten Bereicherung und dem Nutzen für die Universität. Damit verfolgte Bartling die Absicht, die Wahrscheinlichkeit der Reisegenehmigung zu erhöhen sowie groß angelegte Forschungsreisen auch auf Dauer zu etablieren. Deutlich erkennbar wird dies an den Passagen seiner Schreiben, in denen er die Exkursion als wichtigen und notwendigen Bestandteil botanischen Erkenntnisgewinns hervorhob. Forschungsreisen waren laut Bartling zentral, um Lücken in der Kenntnis zu schließen, das Wissen zu erweitern sowie die botanischen Ansichten zu schärfen und zu berichtigen. Die Wahl seines jeweiligen Reiseziels begründete er vor allem damit, dass die Region bisher wenig bereist und untersucht worden sei. In den von Bartling abgefassten Reiseberichten wurden die vorab formulierten Absichten dann immer noch einmal aufgegriffen und deren Erreichen bestätigt. Detailliert beschrieb er auch den Verlauf der Route und die bereisten Regionen. ${ }^{185}$ Der Abschluss jedes Berichts stellte die Darlegung der Ergebnisse der Reise und eine Auflistung der akquirierten Objekte dar. ${ }^{186}$

UniA GÖ, Kur., 5592, Schreiben Bartlings an das Universitätskuratorium, 6.11.1849;

UniA GÖ, Kur., 5592, Schreiben Bartlings an das Universitätskuratorium, 21.3.1851;

UniA GÖ, Kur., 5592, Schreiben Bartlings an das Universitätskuratorium, 16.11.1852.

Auch die von Lantzius-Beninga durchgeführte Forschungsreise nach Ostfriesland im Jahr 1847 hatte zum Ziel, die Sammlung einheimischer Gewächse des Herbariums zu vergrößern. Vgl. UniA GÖ, Kur., 5592, Schreiben Bartlings an das Universitätskuratorium, 14.11.1848.

184 Aus einem Schreiben Bartlings geht zum Beispiel hervor, dass er sich vor Antritt einer Exkursion auch mit der Sprache und den Gepflogenheiten des Reiselandes auseinandersetzte sowie sich Kenntnisse über die Vegetation verschaffte. Vgl. UniA GÖ, Kur., 5584, Schreiben Bartlings an den Geheimen Kabinettsrat, 31.7.1843.

185 Die genaue Beschreibung des Sammelorts sollte darüber hinaus, wie Larsen anmerkt, dem wissenschaftlichen Fund Validität verleihen. Vgl. Larsen 1996, S. 362 f.

186 Teilweise wurden den Schreiben auch Verzeichnisse mit den wissenschaftlichen Bezeichnungen des Gesammelten beigelegt. Vgl. UniA GÖ, Kur., 5584, Bericht über die von dem Professor Bartling in den Monaten September und Oktober 1840 durch einen Teil der österreichischen 
Nicht nur um das Kuratorium von der Notwendigkeit der Reiseunternehmungen zu überzeugen, sondern um diese auch gewinnbringend zu nutzen, verbanden gerade die größer angelegten Reisen Bartlings darüber hinaus vielseitige Interessen: Neben dem botanischen Garten und dem Herbarium wurden fast immer auch andere Göttinger naturhistorische Institutionen mit bedacht. ${ }^{187}$ Gesammelt wurden nicht nur Pflanzen und Sämereien, sondern auch andere Naturprodukte und Tiere, die häufig an das Museum abgegeben wurden. ${ }^{188}$ Gleichzeitig wurde fast jede Reise auch für den Besuch von Fachkolleg*innen sowie botanischen Einrichtungen genutzt. Damit kam Bartling als Vorsteher des Herbariums auch seiner Aufgabe nach, Kontakte ins In- und Ausland zu knüpfen, diese Verbindungen auszubauen und zu pflegen. ${ }^{189}$ Sein Netzwerk hielt er aber nicht nur durch Besuche, sondern auch durch gemeinsames Reisen aufrecht. So fanden Bartlings Exkursionen in Begleitung von Studierenden ${ }^{190}$ wie auch von Kollegen ${ }^{191}$ oder unter der Leitung eines Experten ${ }^{192}$ vor Ort statt. Ebenso konnte der Besuch einer naturwissenschaftlichen Versammlung mit einer Forschungsreise verbunden werden. ${ }^{193}$ Nicht zuletzt boten die Exkursionen

Monarchie gemachten naturwissenschaftlichen Reise, o. D.; UniA GÖ, Kur., 5584, Schreiben Bartlings an das Universitätskuratorium, 11.12.1842; UniA GÖ, Kur., 5584, Schreiben Bartlings an das Universitätskuratorium, 3.1.1844.

${ }^{187}$ Ein Schreiben belegt darüber hinaus einen Erwerb für die Bibliothek. UniA GÖ, Kur., 5584,

Bericht über die von dem Professor Bartling in den Monaten September und Oktober 1840 durch einen Teil der österreichischen Monarchie gemachten naturwissenschaftlichen Reise, o. D.

188 Vor allem die zoologische Sammlung unter der Aufsicht des Hofrats Arnold Adolf Berthold (1803-1861) wurde mit Insekten sowie Land- und Süßwasserconchylien bedacht. Vgl. UniA GÖ, Kur., 5584, Schreiben Bartlings an das Universitätskuratorium, 19.10.1838; UniA GÖ, Kur., 5584, Schreiben Bartlings an das Universitätskuratorium, 5.2.1841; UniA GÖ, Kur., 5584, Schreiben Bartlings an das Kabinett Seiner Majestät des Königs, 29.2.1848.

189 Bei dieser in den Instruktionen für den Direktor explizit formulierten Aufforderung handelte es sich selbstverständlich um keine Neuerung per se. Die Netzwerke unter Botaniker*innen waren bereits im 18. Jahrhundert stark ausgebaut. Vgl. Dauser et al. 2008. Neu war, dass der Austausch nun nicht mehr unter Individuen allein stattfand, sondern von einer zentralen Institution ausgeführt wurde. Vgl. Müller-Wille 2008, S. 88. Diese offizielle Korrespondenz blieb dem Vorsteher des Herbariums vorbehalten und musste nicht durch persönliche Mittel bestritten werden. So geht aus den Rechnungen über die Einnahmen und Ausgaben hervor, dass der dem Herbarium zur Verfügung gestellte Fonds auch für Brief- und Paketsendungen genutzt wurde.

190 Vgl. UniA GÖ, Kur., 5584, Schreiben Bartlings an das Universitätskuratorium, 8.12.1830.

191 Vgl. 2 Cod. Ms. philos. 182: F. G. Bartling, Brief von Friedrich Gottlieb Bartling an Ernst Hampe, 27.2.1848; UniA GÖ, Kur., 5584, Bericht über die von dem Professor Bartling in den Monaten September und Oktober 1840 durch einen Teil der österreichischen Monarchie gemachten naturwissenschaftlichen Reise, o. D.

192 Vgl. UniA GÖ, Kur., 5584, Schreiben Bartlings an das Kabinett Seiner Majestät des Königs, 29.2.1848.

193 Vgl. UniA GÖ, Kur. Pers., 5828, Schreiben Bartlings an den Kanzleirat, 20.11.1834. 
Bartling aber auch eine Auszeit von seinem Göttinger Alltag und ermöglichten es ihm, sich geistig und körperlich zu erfrischen. ${ }^{194}$

Doch auch wenn Bartling die Amtswege genau befolgte und vieles bereits vorausgeplant gewesen zu sein schien, veränderte er seine Pläne auch immer wieder: Nicht nur wurden die Routen abgewandelt, sondern teilweise sogar das gesamte Reiseziel gewechselt. ${ }^{195}$ Nicht selten geschah dies aufgrund der Wetterlage. ${ }^{196}$ Die Einbindung in die Lehre und in die Unterhaltung des botanischen Gartens machte es fast unmöglich, einen anderen Zeitpunkt als die Herbstferien für größere Exkursionen zu nutzen. ${ }^{197}$ Für die Abänderungen wurden keine neuen Anträge formuliert, sondern die Genehmigung erst im Nachhinein eingeholt oder eine Erklärung in den Reiseberichten gegeben.

Mit Blick auf die botanischen Sammlungen verfolgte Bartling als Vorsteher vor allem zwei Ziele: Zum einen ging er seinen persönlichen Forschungsinteressen und dem danach ausgerichteten Sammeln, zum anderen dem Sammeln im Sinne des Herbariums nach. Vordergründiges Ziel war auch auf den Exkursionen die Vervollständigung der Sammlungen. Bartling war aber besonders interessiert daran, das Vorkommen und die Verbreitung von Pflanzengruppen im Zusammenspiel mit den Lokalverhältnissen wie Boden, Bewässerung und Klima zu untersuchen. ${ }^{198}$ Aus diesem Grund wurden manche Regionen wie beispielsweise Fiume (heute Rijeka) in Kroatien auch wiederholt und zu einer anderen Jahreszeit von ihm besucht. ${ }^{199}$ Auch das Bereisen unterschiedlicher Ausläufer der Alpen wird vor diesem Hintergrund noch einmal mehr verständlich. In seinen Berichten gegenüber dem Kuratorium führte Bartling es dann als wichtigen Erfolg an,

194 Vgl. UniA GÖ, Kur., 5584, Schreiben Bartlings an das Universitätskuratorium, 13.8.1842; UniA GÖ, Kur., 5584, Schreiben Bartlings an den Geheimen Kabinettsrat, 31.7.1843. Diese häufige Verknüpfung von intellektueller Arbeit und körperlicher Erholung während des Sammlungsprozesses benennt auch Anne Larsen. Vgl. Larsen 1996, S. 360 f.

195 Vgl. UniA GÖ, Kur., 5584, Schreiben Bartlings an das Universitätskuratorium, 28.1.1841; UniA GÖ, Kur., 5584, Schreiben Bartlings an das Universitätskuratorium, 11.12.1842; UniA GÖ, Kur., 5584, Schreiben Bartlings an den Geheimen Kabinettsrat, 31.7.1843.

${ }^{196}$ Der Einfluss des Wetters und der Jahreszeit auf das Sammeln sowie auf die Reiseroute wird in jedem überlieferten Reisebericht Bartlings thematisiert.

${ }^{197}$ Eine Ausnahme stellt die von Grisebach im Jahr 1839 unternommene Reise nach Albanien dar. Für die ca. sechs Monate andauernde Exkursion bewilligte das Kuratorium ihm sogar ein Reisestipendium von 300 Talern. Vgl. UniA GÖ, Kur. Pers., 4973, Schreiben Grisebachs an königliche Majestät, 24.12.1838; UniA GÖ, Kur. Pers., 4973, Schreiben des Universitätskuratoriums an Grisebach, 28.1.1839.

198 Vgl. UniA GÖ, Kur., 5584, Schreiben Bartlings an das Universitätskuratorium, 9.9.1832.

${ }^{199}$ In dem Reisebericht aus dem Jahr 1840 schreibt Bartling: „Hier blieb denn auch der Unterzeichnete mit seinen Reisegenossen um so lieber einige Zeit, je interessanter es ihm seyn musste, die Flora von Fiume, die er vor mehr als 20 Jahren im Sommer untersucht, und über welche er später Verschiedenes bekannt gemacht hatte, auch im herbstlichen Gewande zu sehen.“ UniA GÖ, Kur., 5584, Bericht über die von dem Professor Bartling in den Monaten September und Oktober 1840 durch einen Teil der österreichischen Monarchie gemachten naturwissenschaftlichen Reise, o. D. 
konnte er bisher nicht bekannte Standorte einer Pflanzenart ausfindig machen. ${ }^{200}$ Ein ganz persönliches Interesse hatte Bartling aber an den Kryptogamen. Die diesen zuzurechnenden Flechten bezeichnete er sogar als seine „Lieblinge“201.

Für den Tauschhandel des Instituts sammelte Bartling vor allem Alpenpflanzen, die sich für diesen Zweck offenbar besonders eigneten:

\begin{abstract}
„Obgleich die im hiesigen botanischen Garten befindliche Sammlung von Alpenpflanzen nicht unbedeutend ist, so dürfte doch eine Vermehrung derselben um so erwünschter seyn, je mehr diese Gewächse einerseits in wissenschaftlicher Rücksicht vorzüglich intressant, andererseits aber deshalb besonders wichtig sind, da sie bei geringen Culturkosten mehr fast als irgend eine andere Abtheilung von Pflanzen die Mittel zur Unterhaltung ausgedehnter Tauschverbindungen, besonders mit den englischen und holländischen Gärten an die Hand geben. “202
\end{abstract}

Aus Bartlings Reiseberichten geht hervor, dass er stets mehrere Exemplare einer Art zusammentrug. Die überflüssigen Dubletten konnten dann nach seiner Rückkehr gegen gleichwertige Pflanzen anderer Personen und Institutionen eingetauscht werden. ${ }^{203}$ Auch wurde der Kontakt zu Fachkolleg*innen während der Exkursionen gelegentlich zum Ankauf von Pflanzen genutzt. ${ }^{204}$

Anders als die Ankäufe erlaubte das selbst durchgeführte Sammeln, die Objekte auch den eigenen Bedürfnissen entsprechend zuzurichten. In welchem Zustand sich die angekauften Pflanzenexemplare genau befanden, ist zwar nicht bekannt, doch war der Trocknungsprozess in jedem Fall bereits abgeschlossen. Selbst wenn die Objekte also noch nicht auf Papier fixiert waren, so waren sie vor dem Trocken doch drapiert worden. ${ }^{205}$ Bartling konnte zwar vor Kaufabschluss die Sammlung sichten - welcher Teil der Pflanze gesammelt und wie dieser aufbereitet wurde, war zu diesem Zeitpunkt aber selbstverständlich nicht mehr zu beeinflussen. ${ }^{206}$ Das Sammeln auf Exkursionen bot diesbezüglich sehr viel

200 Vgl. UniA GÖ, Kur., 5584, Schreiben Bartlings an das Universitätskuratorium, 23.20.1838.

2012 Cod. Ms. philos. 182: F. G. Bartling, Brief von Friedrich Gottlieb Bartling an Ernst Hampe, 27.2.1848.

202 UniA GÖ, Kur. Pers., 5828, Schreiben Bartlings an das Universitätskuratorium, 30.7.1838.

${ }^{203}$ Es ist allerdings naheliegend, dass Bartling auch deshalb mehrere Exemplare einer Art sammelte, um etwaige Verluste auf dem Transportweg zu kompensieren.

204 Vgl. UniA GÖ, Kur. Pers., 5828, Schreiben Bartlings an den Kanzleirat, 20.11.1834; UniA GÖ, Kur., 5584, Schreiben Bartlings an das Universitätskuratorium, 11.12.1842.

205 Eine Bearbeitung des Pflanzenmaterials war nach dem Kauf nur eingeschränkt möglich. Eine Rechnung vom 26. Juni 1852 zeigt, dass im Fall der Sammlung Philippi zumindest das entsprechende Papier und die Etiketten wie üblich beim Göttinger Lithografen Rittmüller bestellt wurden. Vgl. UniA GÖ, Kur., 5592, Rechnung über Einnahme und Ausgabe für das Universitäts-Herbarium zu Göttingen vom 1. Juli 1851 bis dahin 1852.

${ }^{206}$ So sichtete Bartling das Herbarium Philippi vor dem Ankauf persönlich. In seinem Bericht an das Kuratorium schrieb er: „In schuldiger Befolgung des hohen Rescripts vom 27. Februar d. J. [...] hat der ehrerbietigst Unterzeichnete nach dem Schlusse der Vorlesungen sich nach Cassel begeben, die in Frage stehenden Sammlungen nochmals untersucht [...]“. UniA GÖ, Kur., 5600, Schreiben Bartlings an das Universitätskuratorium, 7.4.1846. Dass es durchaus von 
weitreichendere Möglichkeiten. Und auch wenn keines der Notizbücher, die Bartling auf seinen Reisen mit sich führte, erhalten ist, so geben die von ihm verfassten Reiseberichte doch teilweise Aufschluss über sein Vorgehen. ${ }^{207}$ Über das Sammeln selbst und die zum Einsatz gekommenen Materialien gibt es keine Hinweise. ${ }^{208}$ Deutlich wird jedoch, dass das Gesammelte bereits auf der Reise verarbeitet wurde. ${ }^{209}$ Die Pflanzenexemplare wurden getrocknet, geordnet, bezeichnet und danach verpackt. ${ }^{210}$ Je nach Reiseroute und Objektmenge und vor dem Hintergrund, dass man sich zu Fuß fortbewegte, wurden die Pflanzenpakete in Kisten verpackt und noch während der Exkursion nach Göttingen abgeschickt. ${ }^{211}$ Nach der Rückkehr und vor Abfassen des Reiseberichtes wurde

Bedeutung war, welche Teile der Pflanze gesammelt worden waren, beschreibt Larsen beispielhaft: „Botanical specimens consisted of the entire plant, including the roots, bulbs or rhizomes, whenever possible. Young plants with their cotyledons (seed leaves) still visible were important for identification because the embryo often revealed relationships that were hidden in the mature plant. The various stages of the flower were also useful." Larsen 1996, S. 366.

207 Dass es solche Notizbücher gegeben hat, wird deutlich, da Bartling diese an mehreren Stellen explizit benennt und auf diese verweist. Auch die umfangreichen Beschreibungen der bereisten Landschaften und der Reiserouten in seinen Berichten an das Kuratorium lassen darauf schließen, dass Bartling seine Exkursionen umfangreich dokumentierte. Vgl. UniA GÖ, Kur. Pers., 5828, Schreiben Bartlings an den Kanzleirat, 20.11.1834; UniA GÖ, Kur., 5584, Schreiben Bartlings an das Universitätskuratorium, 28.1.1841; UniA GÖ, Kur., 5584, Schreiben Bartlings an den Kanzleirat, 5.2.1841; 2 Cod. Ms. philos. 182: F. G. Bartling, Brief von Friedrich Gottlieb Bartling an Ernst Hampe, 27.2.1848. Ein umfassenderes Verständnis für die weiterhin wenig beachtete materielle Kultur des Sammelns und besonders des Organisierens, Verpackens und Transportierens botanischer Sammlungsobjekte kann von dieser Seite aus somit nicht erfolgen. Für Arbeiten, die einen solchen Weg verfolgen vgl. Laird \& Bridgman 2014, te Heesen 2000; te Heesen 2004.

208 Es ist allerdings davon auszugehen, dass Bartling genauso wie andere Botaniker*innen zumindest mit einer sogenannten ,botany box'zum Aufbewahren des Gesammelten, einem Messer und einer Pflanzenkelle sowie Papier zum Trocknen der Pflanzen ausgestattet gewesen ist. Auch war es üblich, Bestimmungsbücher mit sich zu führen. Vgl. Larsen 1996, S. 366. Einige Materialien wurden auch auf der Reise gekauft. Vgl. UniA GÖ, Kur., 5584, Bericht über die von dem Professor Bartling in den Monaten September und Oktober 1840 durch einen Teil der Österreichischen Monarchie gemachten naturwissenschaftlichen Reise, o. D.

209 Vgl. UniA GÖ, Kur., 5584, Bericht über die von dem Professor Bartling in den Monaten September und Oktober 1840 durch einen Teil der österreichischen Monarchie gemachten naturwissenschaftlichen Reise, o. D.

210 Vgl. UniA GÖ, Kur., 5584, Schreiben Bartlings an das Universitätskuratorium, 29.2.1848. Manche Arbeiten wurden allerdings auch abgegeben. Das Einlegen von Pflanzen übernahm beispielsweise der Pharmazeut Sander. Vgl. UniA GÖ, Kur., 5592, Schreiben Bartlings an das Universitätskuratorium, 19.12.1836. Aus dem Inventarium des Rechnungsjahres 1851/52 geht hervor, dass das Herbarium für das selbstständige Trocknen spätestens seit 1851 eine eigene Drahtpresse besaß. Vgl. UniA GÖ, Kur., 5592, Rechnung über Einnahme und Ausgabe für das Universitäts-Herbarium zu Göttingen vom 1. Juli 1851 bis dahin 1852.

211 Vgl. UniA GÖ, Kur. Pers., 5828, Schreiben Bartlings an den Kanzleisekretär, 19.10.1838. Welche Transportmöglichkeiten für gewöhnlich genutzt wurden, ist nicht überliefert. Lediglich einmal erwähnt Bartling, dass das Gesammelte einem Bekannten mitgegeben wurde. Vgl. UniA GÖ, Kur., 5584, Bericht über die von dem Professor Bartling in den Monaten September und Oktober 1840 durch einen Teil der österreichischen Monarchie gemachten naturwissen- 
das Material von Bartling gesichtet und geordnet. ${ }^{212}$ Die lebenden Pflanzen wurden zeitnah im botanischen Garten kultiviert und die gesammelten Sämereien zum Teil ausgesät oder für den Tauschhandel genutzt. Die getrockneten Pflanzen gelangten in das Herbarium und wurden dort untersucht und als Herbarbelege zugerichtet.

Auch wenn die auf Exkursionen gesammelten Pflanzenexemplare den eigenen Ansprüchen und Wertmaßstäben vermutlich deutlich mehr entsprochen haben werden und das vorausschauende Sammeln von Dubletten sich in der Folge sicherlich als nützlich erwies, brachte das Akquirieren auf Reisen Bartling finanziell genauso in Bedrängnis wie der Erwerb durch Kauf. Denn wie die naturhistorischen Museen der Zeit besaß das Institut keinen eigenen Reiseetat. ${ }^{213}$ Und obwohl die Exkursionen sowohl dem Herbarium als auch dem botanischen Garten zugutekamen, sollten sie allein durch den Fonds des Herbariums bestritten werden. Dass diese Regelung Bartling vor große Herausforderungen stellen musste, geht ganz deutlich aus einem Vorschlag hervor, den er 1847 dem Kuratorium unterbreitete. ${ }^{214}$ In diesem spricht er sich - prognostizierend, dass der Etat von 100 Talern auch in den kommenden Jahren nicht ausreichen werde dafür aus, den häufig nicht ausgeschöpften Fonds des Gartens für Exkursionen mobil zu machen. ${ }^{215}$ Diese Umverteilung lehnte das Kuratorium allerdings ab, sodass Bartling zwar in der Folge gelegentlich außerordentliche Zuschüsse aus der Etatsumme des Gartens entnehmen durfte, dafür aber weiterhin eine Antragstellung vornehmen musste. ${ }^{216}$ Flexibles Handeln war somit auch hinsichtlich der Forschungsreisen und kleineren Exkursionen nicht möglich. Dabei zeigen die Trennung der Fonds sowie deren unterschiedliche Höhe, dass das Herbarium gegenüber dem botanischen Garten eine weniger gestärkte Position bekleidete. Die Forderung, von dem weniger umfangreichen Etat des Herbariums neben den Ausgaben für Pflanzenankäufe, den Hilfsarbeiter, Heiz- und Reinigungskosten, Materialien und Mobiliar auch die unternommenen Reisen des Vorstehers zu decken, stützt darüber hinaus die Aussage, Bartling sei der erste Göttinger Botaniker gewesen, der selbst umfangreiche Forschungsreisen außerhalb Mitteleuropas unternahm. ${ }^{217}$ Von den sechs größeren Exkursionen, die Bartling sich in

schaftlichen Reise, o. D. Eine Herausforderung für den Transport stellten besonders die Sämereien dar, die bei Feuchtigkeit schnell zu keimen drohten. Vgl. UniA GÖ, Kur. Pers., 4973, Schreiben Grisebachs an das Universitätskuratorium, 9.2.1839. Sie wurden, wie Larsen beschreibt, deshalb häufig in Sand oder gar Rosinen gelegt, die ihnen die Feuchtigkeit entziehen sollten. Vgl. Larsen 1996, S. 367.

212 Vgl. UniA GÖ, Kur., 5584, Schreiben Bartlings an das Universitätskuratorium, 3.1.1844;

UniA GÖ, Kur., 5584, Schreiben Bartlings an das Universitätskuratorium, 29.2.1848.

${ }^{213}$ Vgl. Kretschmann 2006, S. 175.

${ }^{214}$ Vgl. UniA GÖ, Kur., 5592, Schreiben Bartlings an das Universitätskuratorium, 15.2.1847.

215 Vgl. ebd. Dem Garten standen jährlich 2.980 Taler zu.

216 Vgl. UniA GÖ, Kur., 5592, Schreiben des Universitätskuratoriums an Bartling, 28.4.1847.

217 Vgl. Wagenitz 1996, S. 11. 
2 Eine historische Ethnografie und Praxeografie

den Jahren von 1832 bis 1852 durch das Kuratorium genehmigen ließ, dauerten nur eine weniger als sechs Wochen und zwei insgesamt zwei Monate an. ${ }^{218}$ Reisen wurden auch zuvor unternommen, aber offensichtlich nicht in der Dichte, in einem deutlich kleineren Rahmen und vor allem durch private Mittel gedeckt. ${ }^{219}$ Die finanzielle Beschränkung führte allerdings in keiner Weise zur Abnahme von Bartlings Reiseaktivitäten. Vielmehr ließ er sich seine Reisen im Nachhinein genehmigen, überschritt sogar den Fonds und bat, fielen die Reise-kosten doch höher als erwartet aus, nachträglich um Zuschüsse. ${ }^{220}$

\section{Tausch}

Die Vermutung, dass sich ein regelmäßigeres und umfangreicheres Akquirieren von Pflanzenexemplaren auf Reisen erst mit der Gründung des Herbariums und auf die Initiative Bartlings hin entwickelte, wird durch zwei Aussagen Bartlings selbst bestätigt: Im Bericht zum Rechnungsjahr 1849/50, welchen Bartling noch einmal gezielt nutzte, um den Stellenwert des Herbariums zu bekräftigen, äußerte er sich auch zu den zur Erhaltung und Erweiterung der Sammlung notwendigen Strategien.

\footnotetext{
„Was die Herbeischaffung der in die Sammlung aufzunehmenden Gegenstände betrifft, so wird zwar, wie bisher, vieles durch Ankauf oder gelegentlichen Tausch erworben werden müssen, manches aber [...] auch fernerhin nur durch eigenes Sammeln auf Excursionen und Reisen zu erlangen seyn.“"221
}

Auch seine Pläne für eine Exkursion ins südliche Tirol im Jahr 1838 versah er mit einer Begründung, die auf das Verhältnis der Sammelstrategien untereinander rückschließen lässt:

218 Bartling ging alle zwei bis vier Jahre auf größere Forschungsreise. Daneben unternahm er kleinere Exkursionen im Königreich Hannover.

${ }^{219}$ Die von Schrader unternommenen Reisen dauerten beispielsweise nur etwa acht Tage an und fanden meist innerhalb des Landes statt. Sowohl in seinen Anfragen an das Kuratorium als auch in den darauf erfolgten Urlaubsbewilligungen finden sich keine Erläuterungen zum Reiseziel oder dem Zweck der Exkursionen. Auch finanzielle Zuschüsse wurden von Schrader nicht beantragt. Vgl. UniA GÖ, Kur. Pers., 4949, Schreiben aus Hannover an Schrader, 28.9.1814; UniA GÖ, Kur. Pers., 4949, Schreiben des Universitätskuratoriums an Schrader, 7.10.1819; UniA GÖ, Kur. Pers., 4949, Schreiben Schraders an das Universitätskuratorium, 12.9.1822; UniA GÖ, Kur. Pers., 4949, Schreiben Schraders an das Universitätskuratorium, 22.9.1824.

${ }^{220}$ Vgl. UniA GÖ, Kur., 5584, Schreiben Bartlings an den Kanzleirat, 3.11.1840; UniA GÖ, Kur., 5584, Schreiben Bartlings an das Universitätskuratorium, 11.12.1842; UniA GÖ, Kur., 5584, Schreiben Bartlings an das Kabinett Seiner Majestät des Königs, 29.2.1848; UniA GÖ, Kur., 5592, Schreiben des Universitätskuratoriums an Bartling, 22.12.1852; UniA GÖ, Kur., 5592, Schreiben Bartlings an das Universitätskuratorium, 4.10.1853.

221 UniA GÖ, Kur., 5592, Schreiben Bartlings an das Universitätskuratorium, 21.3.1851. 
„Eine solche Vermehrung ist jedoch auf dem gewöhnlichen Wege nicht wohl zu erreichen, da selbst den süddeutschen Gärten viele seltene Alpenpflanzen fehlen, und nur durch Sammeln in den Alpen selbst zu bewerkstelligen [...]."222

Die Durchführung von Forschungsreisen stellte demnach nicht den ,gewöhnlichen Weg' dar, auf dem Pflanzen für die Sammlungen gewonnen wurden. Zu diesem gehörten vielmehr der Ankauf sowie der Tausch. Welchen Umfang der Tauschhandel im Verhältnis zum Kauf einnahm, ist jedoch schwer festzustellen. Anders als die käufliche Akquisition und ein großer Teil der Forschungsreisen musste er dem Kuratorium gegenüber nicht dokumentiert werden. Seine Wirksamkeit für die Sammlung in der Folge zu verkennen, dürfte sich aber als Fehlschluss herausstellen, denn die bisherigen Untersuchungen zu den Gelehrtennetzwerken des 18. Jahrhunderts haben sehr deutlich gemacht, welches Gewicht der (wissenschaftlichen) Vernetzung auch für den Austausch von Objekten zukam. Trotz des heutigen Fehlens einer umfangreichen Korrespondenz Bartlings und damit von Dokumenten, die die Tauschgeschäfte des Göttinger Herbariums ausführlich dokumentieren könnten, sollte ihre Bedeutsamkeit für die Sammlung nicht unterschätzt werden. Die Tatsache, dass es für sie offensichtlich keiner Genehmigung bedurfte, lässt vielmehr einen regen Nutzen dieser Form der Akquisition vermuten, die sich aber weniger auf bürokratischer Ebene abdrückte.

Dass dem Tauschhandel tatsächlich eine zentrale Bedeutung zugekommen sein muss, zeigt sich an seinem zentralen Tauschobjekt, der Dublette. Dubletten, jene Objekte also, die mehrfach in der Sammlung vorhanden waren, wurden genauso wie dies die naturhistorischen Museen des 19. Jahrhunderts taten gegen andere Pflanzenexemplare eingetauscht. ${ }^{223}$ Dabei wurden diese für die eigene Sammlung als entbehrlich eingestuften Pflanzenexemplare in Göttingen ganz gezielt eingeplant. Sowohl das Sammeln von Dubletten als auch das Aussäen von Pflanzen, die in der Folge im Tausch an andere Gärten abgegeben wurden, zeugen für ein solches Vorausplanen. Wiederholt nennt Bartling außerdem das Vorhandensein von Dubletten als Argument für den Ankauf einer Sammlung.224 Der Gedanke, mehrfach vorhandene Pflanzenexemplare (und auch andere Objekte) so einzutauschen, dass unterschiedliche Sammlungen dadurch ihre Bestände gegenseitig zu ergänzen vermochten, stand mit dem Wunsch nach Vollständigkeit und damit mit den verfolgten Sammlungsidealen in Verbindung. ${ }^{225}$ Gleichzeitig weist Kretschmann auch auf die sich verändernde Bedeutung der Dublette im Verlauf des 19. Jahrhunderts hin:

222 UniA GÖ, Kur. Pers., 5828, Schreiben Bartlings an das Universitätskuratorium, 30.7.1838.

223 Vgl. Kretschmann 2006, S. 151 f.

224 Vgl. UniA GÖ, Kur., 5586, Schreiben Bartlings an das Universitätskuratorium, 17.2.1837;

UniA GÖ, Kur., 5600, Schreiben Bartlings an das Universitätskuratorium, 10.1.1846.

225 Müller-Wille bezeichnet das gezielte Sammeln von Dubletten gar als Konzept, das erst mit Linné Bekanntheit zu erlangen schien. Vgl. Müller-Wille 2017a, S. 121, Fußnote 35. 
„War sie zunächst bedenkenlos gegen andere Objekte eingetauscht worden, so besaß sie nach der Jahrhundertmitte nicht selten einen eigenen Wert. Gerade ihre minimalen Abweichungen machten sie zu einem wichtigen Gegenstand der evolutionstheoretischen Forschung, die eine möglichst große Anzahl ähnlicher Objekte benötigte, um in dem scheinbar Gleichen das Typische wie das Abweichende herauszustellen.“226

Bartlings Vorgehen spiegelt diese Entwicklung allerdings nicht wider. ${ }^{227} \mathrm{Ihm}$ galten Dubletten vielmehr als entbehrliche Objekte, die ohne Dokumentation oder Genehmigung durch das Kuratorium eingetauscht werden durften und, da für sie auch der Fonds nicht bemüht werden musste, eine weitestgehend flexible Akquirierung für das Göttinger Herbarium möglich machten.

Wichtige Voraussetzung für den Handel war jedoch die Äquivalenz der einzutauschenden Pflanzenexemplare, wie ein Auszug aus einem Brief Bartlings an den Apotheker Georg Ernst Ludwig Hampe (1795-1880) von 1848 zeigt:

„Die Grönländischen Pflanzen, von denen Sie mir schreiben, acquirierte ich gern für das Univ. Herbar., doch wird dies davon abhängen, ob Sie hier ein genügendes Aequivalent finden oder nicht. “ 228

Woran genau die Gleichwertigkeit von Pflanzensammlungen oder einzelnen Pflanzenexemplaren gemessen wurde, ist für die unter Bartling durchgeführten Tauschhandel nicht mehr zu bestimmen. Anzunehmen ist jedoch, dass auch hier jene Wertmaßstäbe griffen, die an die angekauften Sammlungen gestellt wurden. ${ }^{229}$ Ebenso war es möglich, lebende Pflanzen gegen getrocknete Exemplare einzutauschen. ${ }^{230} \mathrm{Ob}$ Hampe bei seinem Besuch aber tatsächlich ein Äquivalent für die von ihm angebotene Sammlung fand und ob er überhaupt nach Göttingen gekommen ist, lässt sich nicht belegen. Allerdings wurden laut Katalog im Jahr 1848 insgesamt 100 grönländische Pflanzen für viereinhalb Reichstaler für das Herbarium angekauft. Dabei könnte es sich durchaus um eine zusätzliche,

226 Kretschmann 2006, S. 151.

227 Ein bewusstes Zusammentragen mehrerer Exemplare einer Art ist aber beispielsweise für das auf pflanzengeografische Studien ausgerichtete Herbarium Grisebachs zu belegen.

2282 Cod. Ms. philos. 182: F. G. Bartling, Brief von Friedrich Gottlieb Bartling an Ernst Hampe, 27.2.1848.

229 Auch Müller-Wille und Stelter stufen die Tauschbeziehungen als Äquivalenzbeziehungen ein. Vgl. Müller-Wille 2008, S. 87 f.; Stelter 2018, S. 190-194. Für das 19. Jahrhundert stellt MüllerWille dabei fest: „So verlagerte sich das naturgeschichtliche Interesse ganz auf die Beziehungen unter den gesammelten Gegenständen, dem, was das ,natürliche System` der Lebewesen genannt wurde. Im Vordergrund stand die Frage, auf welcher Ebene des Systems - Art, Gattung, Familie, oder Klasse - Lebewesen als Gleiche füreinander eintreten können. Die Beziehungen unter Sammlern regelten sich auf der Grundlage dieses Systems, ihr Erfolg wurde nach ihrer Fähigkeit bemessen, eine möglichst große Zahl ,neuer', d. h. dem System noch nicht einverleibter Arten zu entdecken." Ebd. S. 88. Deshalb bot sich der Tausch auch vornehmlich für Sammlungen mit ähnlichen Interessen an und behinderte den Austausch mit Institutionen, die andere Ordnungssysteme verfolgten. Vgl. Stelter 2018, S. 192.

230 Vgl. UniA GÖ, Kur., 5592, Schreiben Bartlings an das Universitätskuratorium, 14.12.1840. 
ausgleichende Zahlung handeln, die Bartling neben einzutauschenden Pflanzen zu entrichten hatte. Eine solche Kombination von Sammelstrategien scheint in jedem Fall möglich.

Neben dem Tausch beim Besuch externer Botaniker*innen in Göttingen, wie Bartling ihn Hampe vorschlug, fand diese Form des Handels auch auf seinen Forschungsreisen statt. So berichtete er,

„[...] auf größern und kleinern Excursionen für das Universitäts-Herbarium zu sammeln fortgefahren und die sich darbietenden Gelegenheit[en] zum Eintausch gegen überflüssige

Doubletten und zur Erweiterung der Verbindungen für das Institut benutzt“231

zu haben. Dabei standen die Tauschgeschäfte, wie dieser Auszug sowie der Brief an Hampe zeigen, eng mit der Pflege von Kontakten in Zusammenhang. So wurde die Korrespondenz zur Abwicklung von Tauschgeschäften genutzt. Ob für den Tausch die gleichen Netzwerke aktiviert wurden wie für den Kauf, muss an dieser Stelle allerdings unbeantwortet bleiben. Da nur wenige Tauschgeschäfte im Einzelnen zu belegen sind, kann auch keine Aussage darüber getroffen werden, welche Pflanzen vornehmlich für das Herbarium akquiriert worden sind. Das verstärkte Sammeln von Alpenpflanzen für den Tauschhandel zeigt allerdings, dass zumindest in Hinblick auf das Zusammentragen von Dubletten nachfrageorientiert gehandelt wurde. ${ }^{232}$ Diese Vermutung bestätigt der Austausch von Desideraten- und Dublettenlisten zwischen den an der Korrespondenz beteiligten Personen. ${ }^{233}$ Indem man sich über Pflanzenwünsche und vorhandenes Pflanzenmaterial verständigte, konnte der Tauschhandel vorausgeplant und so zu einer beidseitigen Vervollkommnung der Sammlungen beigetragen werden. Dabei griff sicherlich auch im Herbarium jene partizipatorische Praxis, die Kretschmann für die Prozesse der Akquirierung sowie der Abgabe von Sammlungsobjekten in naturhistorischen Museen beschreibt. ${ }^{234}$ Demnach drücke sich Teilhabe an der Sammlung auch durch den Austausch von Objekten aus. Indem bereits bearbeitetes und bestimmtes Material abgegeben wurde, beabsichtigte man, so Kretschmann, ein genaueres Wissen nach außen zu tragen. Den Dublettenhandel bezeichnet er deshalb als „Multiplikator naturkundlichen Wissens“235.

231 UniA GÖ, Kur., 5592, Schreiben Bartlings an das Universitätskuratorium, 15.9.1835.

232 Vgl. UniA GÖ, Kur., 5592, Schreiben Bartlings an das Universitätskuratorium, 15.2.1847.

233 Ein solcher Austausch ist sowohl für Schrader als auch für Bartling zu belegen. Vgl. 2 Cod. Ms. philos. 182: H. A. Schrader. Die Korrespondenz Bartlings ist zwar nicht in Göttingen überliefert, doch besitzt die Universitätsbibliothek Leipzig 35 Briefe aus dem Schriftwechsel mit Gustav Kunze (1793-1851). Diese konnten zwar nicht eigens eingesehen werden, doch zeigen bereits die Inhaltsbeschreibungen, dass auch der Austausch von Dubletten- und Desideratenlisten wiederholt zum Thema gemacht wurde. Vgl. Briefe von F. G. Bartling an G. Kunze in der Universitätsbibliothek Leipzig.

234 Vgl. Kretschmann 2006, S. 152.

235 Kretschmann 2006, S. 152. 
Eine solche Vermehrung wissenschaftlicher Erkenntnis fand noch einmal mehr durch eine besondere Form des Austauschs von Objekten statt, die als Eigenheit botanischen Tauschhandels verstanden werden kann. Eine wiederkehrende Sammlungspraxis im Herbarium stellte nämlich der Erwerb gegen Beschreibung dar. Dabei zogen externe Botaniker*innen Göttinger Kolleg*innen zu Rate, durch die sie Pflanzenexemplare bearbeiten und bestimmen ließen. In der Folge erhielt die beschreibende Instanz als Gegenleistung die entsprechenden Dubletten. ${ }^{236}$ So bemerkte Bartling in seinem Bericht, den er dem Kuratorium zum Rechnungsjahr 1849/50 vorlegte:

\begin{abstract}
„Nicht unwichtige Beiträge sind dem Universitäts-Herbarium bis jetzt auch noch dadurch erwachsen, daß ich theils für die Untersuchung und Bestimmung der von verschiedenen Reisenden gesammelten Pflanzen [...] als Honorar die untersuchten Exemplare erhalten [...] und da ich selbst botanische Sammlungen nicht besitzen darf, dem UniversitätsHerbarium überwiesen habe. Meine Verhältnisse und Verbindungen werden hoffentlich auch fernerhin Gelegenheit zu ähnlichen Acquisitionen geben, und schon jetzt ist wieder eine sehr bedeutende Sammlung aus Java in meinen Händen, für deren Bearbeitung ich die sämmtlichen zahlreichen Dubletten derselben erhalte.“237
\end{abstract}

Andersherum verschickte auch Bartling Pflanzen zur Begutachtung und Einschätzung. Gerade durch diesen Dienst unter Fachkolleg*innen wurde der Anspruch an die Wertigkeit des für die Sammlung aufzunehmenden Materials noch einmal mehr gesichert und erfüllt.

\title{
IV. Schenkung
}

Schließlich erhielt das Göttinger Herbarium auch Sammlungen als Geschenk. Dabei schien es sich aber um eine seltener zum Einsatz gekommene Sammelstrategie gehandelt zu haben, die auch rar an Nachweisen ist. Abgesehen von einer im Bericht zum Rechnungsjahr 1834/35 erwähnten Sammlung nordamerikanischer Pflanzen, die auf Geheiß des Königs, dem Herzog von Cambridge, gleichmäßig zwischen der Universität Göttingen und dem Königlichen Garten zu Herrenhausen aufgeteilt wurden, handelte es sich hierbei vornehmlich um ein wissenschaftlich motiviertes Schenken unter Fachkolleg*innen. ${ }^{238}$ Anders als bei den naturhistorischen Museen der Zeit wirkte sich am Göttinger Herbarium ein Schenken im Sinne des Memoriagedankens oder eines Statusdenkens weniger stark aus. ${ }^{239}$ Eine auf diese Weise stattfindende fürstliche oder aber bürgerliche Teilhabe an der Sammlung schien für eine Universitätssammlung, die in Göttingen darüber hinaus ja auch dem Kuratorium und damit dem König direkt unterstellt

\footnotetext{
236 Als Dank wurden und werden neue Arten in dem Zuge häufig auch nach Kolleg*innen benannt.

237 UniA GÖ, Kur., 5592, Schreiben Bartlings an das Universitätskuratorium, 21.3.1851.

238 Vgl. UniA GÖ, Kur., 5592, Schreiben Bartlings an das Universitätskuratorium, 15.9.1835.

239 Vgl. Kretschmann 2006, S. 162.
} 
gewesen ist, weniger gegriffen $\mathrm{zu}$ haben. ${ }^{240}$ Es ist davon auszugehen, dass Schenkungen vielmehr beim Besuch externer Wissenschaftler*innen in Göttingen oder bei Bartlings Exkursionen gemacht wurden. ${ }^{241}$ Inwieweit eine Schenkung in Hinblick auf die Erweiterung der Sammlung als wünschenswert betrachtet wurde, lässt sich nicht beantworten. Die stark beschränkte Einflussnahme auf die Wertigkeit des Materials sowie den Gegenstand an sich dürfte aber bei dieser Form des Erwerbs eine Herausforderung gewesen sein. Denn das Ablehnen einer Schenkung konnte sicherlich keine Option darstellen. Zudem bedeuteten umfangreichere Schenkungen auch einiges an Mehrarbeit, wie es am Beispiel der 1850 als Geschenk ans Herbarium gekommenen etwa 1.000 javanischen Gewächse des Pflanzensammlers Friedrich August Carl Waitz (1798-1882) deutlich wird. ${ }^{242}$ Denn dem Nachtrag im Akquisitionskatalog fügte Drude folgende Notiz hinzu: „Die Bestimmungen sind von Bartling theilweise ausgeführt, aber bis jetzt (1878) liegen noch zahlreiche Exemplare völlig unbestimmt" 243 vor.

Auf also insgesamt vier unterschiedlichen Wegen gelangten Pflanzenexemplare in das Göttinger Herbarium. Bei diesen Prozessen der Akquirierung war Bartling zentraler Akteur. Das bedeutet nicht, dass nicht auch andere Dozierende wie Schrader oder Grisebach im Sinne des Instituts handelten, doch den Ausbau des Herbariums im Speziellen trieb vor allem dessen Vorsteher an. Von den aus eigener Hand stammenden Instruktionen gerahmt, die ihm den Besitz einer privaten Sammlung untersagten, ihm gleichzeitig aber das Führen der offiziellen Korrespondenz und der Geschäfte des Herbariums vorbehielten, setzte sich Bartling intensiv für die Erweiterung der Sammlung ein. Dabei beeinflussten Sammelstrategien, Objekte und Sammlung sich gegenseitig. Die einzelnen Abteilungen besaßen nicht nur unterschiedlich hohe Priorität, auch wurden für ihren Zuwachs unterschiedliche Strategien präferiert. Hauptsammlung und Flora Hannoverana nahmen stets eine gesonderte Rolle ein. ${ }^{244}$ Während die allgemeine Sammlung vornehmlich durch Käufe erweitert wurde, kamen der Sammlung ,einheimischer Gewächse besonders die Ergebnisse der selbst durchgeführten Exkursionen zugute. Den Ansprüchen auf eine wissenschaftliche Wertigkeit der Objekte nach Möglichkeit folgend, wurden Pflanzenexemplare und Sammlungen erworben und zunehmend durch eigene Exkursionen gesammelt. Die akquirierten Objekte selbst trugen nicht allein zum Anwachsen der Sammlung bei und gaben dem Herbarium dadurch seine Daseinsberechtigung. Sie nahmen darüber hinaus eine wichtige Rolle im Festigen von Verbindungen zu anderen Personen und

\footnotetext{
240 Vgl. ebd., S. 156 f.

241 Während der Versammlung der Naturforscher*innen in Stuttgart konnte Bartling 1834 nicht nur Sammlungen käuflich erwerben, sondern wurden ihm auch Pflanzen zum Geschenk gemacht. Vgl. UniA GÖ, Kur. Pers., 5828, Schreiben Bartlings an den Kanzleirat, 20.11.1834.

242 Vgl. Wagenitz 1982, S. 173.

243 PWI.Syst.Geobotan.HE2.

244 Die medizinische Abteilung, die ökonomische Botanik und die Drogen fanden in der Dokumentation zu den Akquirierungsprozessen nur gelegentliche und beiläufige Erwähnung.
} 
Institutionen ein. Das Sammeln und Erwerben von Dubletten erweiterte außerdem den Handlungsspielraum der Sammlung und bot ihr in der Folge wiederum neue Möglichkeiten der Akquisition. Anders als der botanische Garten war das Herbarium noch keine etablierte Institution. Das zeigt die Trennung der sich zudem in ihrer Höhe maßgeblich unterscheidenden Fonds sowie die immer wiederkehrenden Erklärungen der Bedeutsamkeit der Institution in Bartlings Schreiben. Das Herbarium musste seine Eigenständigkeit immer wieder neu behaupten. Es ist also nicht verwunderlich, dass Bartling wiederholt in Verhandlung mit dem Kuratorium trat, Vorschläge für Änderungen in den ökonomischen Strukturen machte und damit das Verhältnis von Herbarium und Garten neu zu bestimmen suchte.

Trotz der finanziellen Herausforderungen wuchs der Sammlungsbestand des Herbariums laut Aussage Bartlings bis zum Jahr 1851 auf 1.300 Arten in weit über 100.000 Exemplaren an. ${ }^{245}$ Zu Unterbrechungen des Erwerbs von Pflanzenmaterial kam es jedoch nicht nur dann, wenn der Fonds erschöpft war. Immer wieder erklärte Bartling gegenüber dem Kuratorium, dass die Neuanschaffung gestoppt werden müsste und erst dann wieder aufzunehmen sei, wenn das bisher angesammelte Material fertig bearbeitet wäre. ${ }^{246}$ Dem Kuratorium boten diese regelmäßigen Erklärungen Bartlings über die Verzögerungen im Ausbau der Sammlung offenbar Anlass, den Vorsteher des Herbariums doch einmal nach dem voraussichtlichen Ende der Ordnungsvorgänge zu befragen. In einem Antwortschreiben vom 21. März 1851 holte Bartling weit aus und legte dem Kuratorium eine „[...] ausführliche Vorstellung des Zustandes und

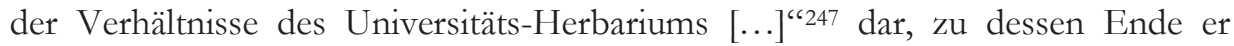
folgendes Fazit zog:

\footnotetext{
„Aus den oben erörterten Umständen dürfte sich nun ergeben, daß mit dem Einordnen der gegenwärtig vorhandenen noch ungeordneten Vorräthe, welches wohl, wenn nichts hinzukäme, in 3-4 Jahren beschafft seyn könnte, die nöthigen Arbeiten für das UniversitätsHerbarium nicht abgeschlossen seyn werden, daß vielmehr, indem stets neues Material zur Vervollständigung desselben zu erwerben seyn wird, dieses Institut fortwährend wenigstens dieselben Arbeitskräfte, wie bisher, in Anspruch nehmen dürfte.“248
}

\footnotetext{
245 Vgl. UniA GÖ, Kur., 5592, Schreiben Bartlings an das Universitätskuratorium, 21.3.1851.

246 Vgl. UniA GÖ, Kur., 5592, Schreiben Bartlings an das Universitätskuratorium, 15.9.1835;

UniA GÖ, Kur., 5592, Schreiben Bartlings an das Universitätskuratorium, 15.4.1844;

UniA GÖ, Kur., 5592, Schreiben Bartlings an das Universitätskuratorium, 6.11.1849.

247 UniA GÖ, Kur., 5592, Schreiben Bartlings an das Universitätskuratorium, 21.3.1851.

248 Ebd.
} 
Genauso wie Kretschmann es für das Berliner Museum für Naturkunde darlegt, bestand auch in Göttingen die Logik der Sammlung folglich in ihrer Dynamik. ${ }^{249}$ Ihr Anwachsen konnte zwar abgebremst werden, doch zu stoppen war es nicht. Von einem bewussten Abschluss der Sammeltätigkeit und Akquisition, wie vom Kuratorium in Hannover angedacht, war erst recht nicht auszugehen. ${ }^{250}$

${ }^{249}$ Vgl. Kretschmann 2006, S. 155. Das Berliner Museum für Naturkunde stand jedoch vor einem anderen Problem: Sein Direktor Martin Heinrich Karl Lichtenstein (1780-1857) sah sich einer regelrechten Überfüllung und kaum einzuschränkenden Vermehrung der Sammlung ausgesetzt. Vgl. ebd.

250 Auch Hans Peter Hahn betont, dass der Prozess des Sammelns nie als beendet anzusehen ist. Sehr anschaulich stellt er dabei heraus, dass die Unabgeschlossenheit von Sammlungen vielmehr ein wichtiges Charakteristikum darstellt, das eine Offenheit für neue Fragestellungen überhaupt erst möglich macht. Vgl. Hahn 2018. 


\subsection{Mit Herbarbelegen forschen}

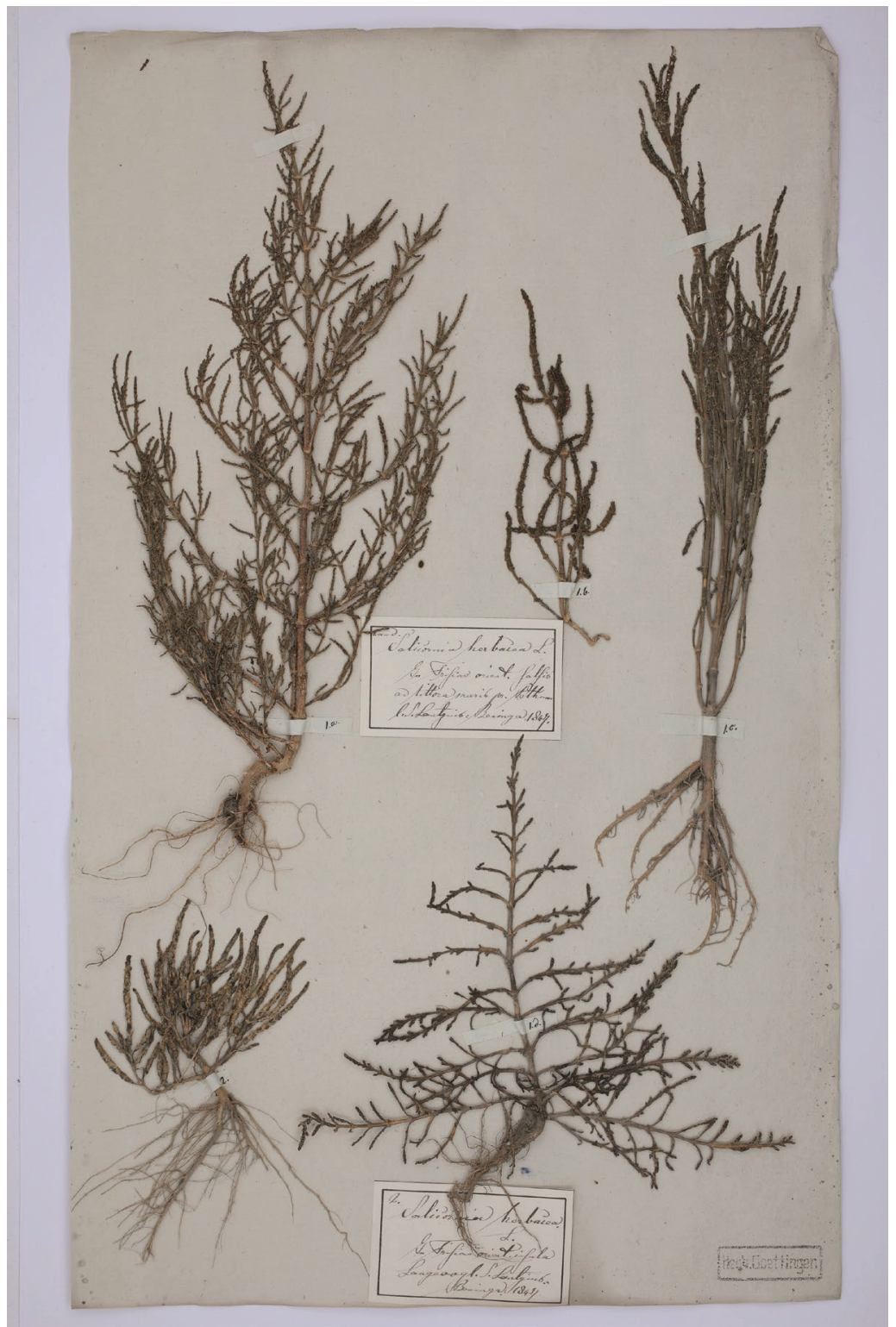

Abb. 11: Herbarbeleg von Salicornia europaea L., gesammelt von B. S. G. Lantrius-Beninga im Jabr 1847 am Meer bei Petkum (1 a-d) sowie auf der Insel Langeoog (2), Herbarium Göttingen, obne Inventarnummer. 
Nur ein Bruchteil der im Göttinger Herbarium vorhandenen Pflanzenbelege sind bisher digitalisiert worden. Vor allem die wertvollen Typusexemplare sind für die heutige Forschung von Bedeutung und liegen als Scans in der Datenbank des Instituts vor. Die meisten Bögen hingegen werden ohne zusätzlich hinterlegtes Digitalisat in den Schränken der Sammlung nach Gattungen sortiert aufbewahrt. Zu dieser Objektgruppe gehören auch die hier gezeigten Exemplare von Salicornia herbacea L. (Salicornia europaea L.). ${ }^{251}$

Gleich zwei Exemplare in fünf Sprossen wurden auf dem Papierbogen fixiert. Die Art der Zurichtung und Beschriftung gleicht dabei den von Bartling erstellten Pflanzenbelegen: Die zwei Etiketten wurden auf gleiche Weise mit einem schwarzen Rahmen versehen und tragen den wissenschaftlichen Namen sowie Informationen zu Fundort, Sammler und Sammeldatum. Zur Vermeidung von Verwechslungen wurden die Exemplare auch hier durchnummeriert und die Klebestreifen zum Fixieren der Präparate mit der jeweiligen Zahl versehen, sodass Etikett und Pflanze aufeinander verweisen. Da vom ersten Pflanzenexemplar vier Stränge gesammelt worden sind, wurden diese darüber hinaus mit einem Buchstaben gekennzeichnet. Denn während die Präparate 1. a-d in Petkum gesammelt wurden, stammt das Präparat 2. von der Insel Langeoog.

Alle Exemplare sind jedoch auf die 1847 von Bojung Scato Georg LantziusBeninga (1815-1871), dem Assistenten am Herbarium, durchgeführte Exkursion nach Ostfriesland zurückzuführen. Die die Etiketten zierende Handschrift lässt sich ihm zweifelsfrei zuordnen. ${ }^{252}$ Aufgrund seiner Assistentenstelle am Herbarium ist außerdem davon auszugehen, dass Lantzius-Beninga nicht nur die Etiketten erstellte, sondern den gesamten Beleg herrichtete. Die Pflanzenart Salicornia herbacea selbst geht jedoch nicht auf ihn zurück, sondern ist bereits 1762 von Linné beschrieben worden. In Lantzius-Beningas Beiträgen zur Kenntniss der Flora Ostfrieslands 253 wird sie in der Beschreibung der Flora der Marsch als eine jener Arten genannt, die vor allem unmittelbar an der Küste auftreten. Salicornia herbacea, von ihm auch als „Glasschmalz“254 oder „Sülte“255 bezeichnet, sei

„[d]ie erste Pflanze, welche sich in Masse auf dem angeschlemmten, noch nackten Boden zeigt" ${ }^{\text {"256. }}$.

\footnotetext{
${ }^{251}$ Der Scan wurde auf Anfrage durch den Kurator der Sammlung angefertigt.

252 Vgl. UniA GÖ, Kur. Pers., 5909; Wagenitz 1982, S. 204.

253 Vgl. Lantzius-Beninga 1849.

254 Ebd., S. 43.

255 Ebd., S. 25 u. 43.

256 Ebd., S. 25.
} 


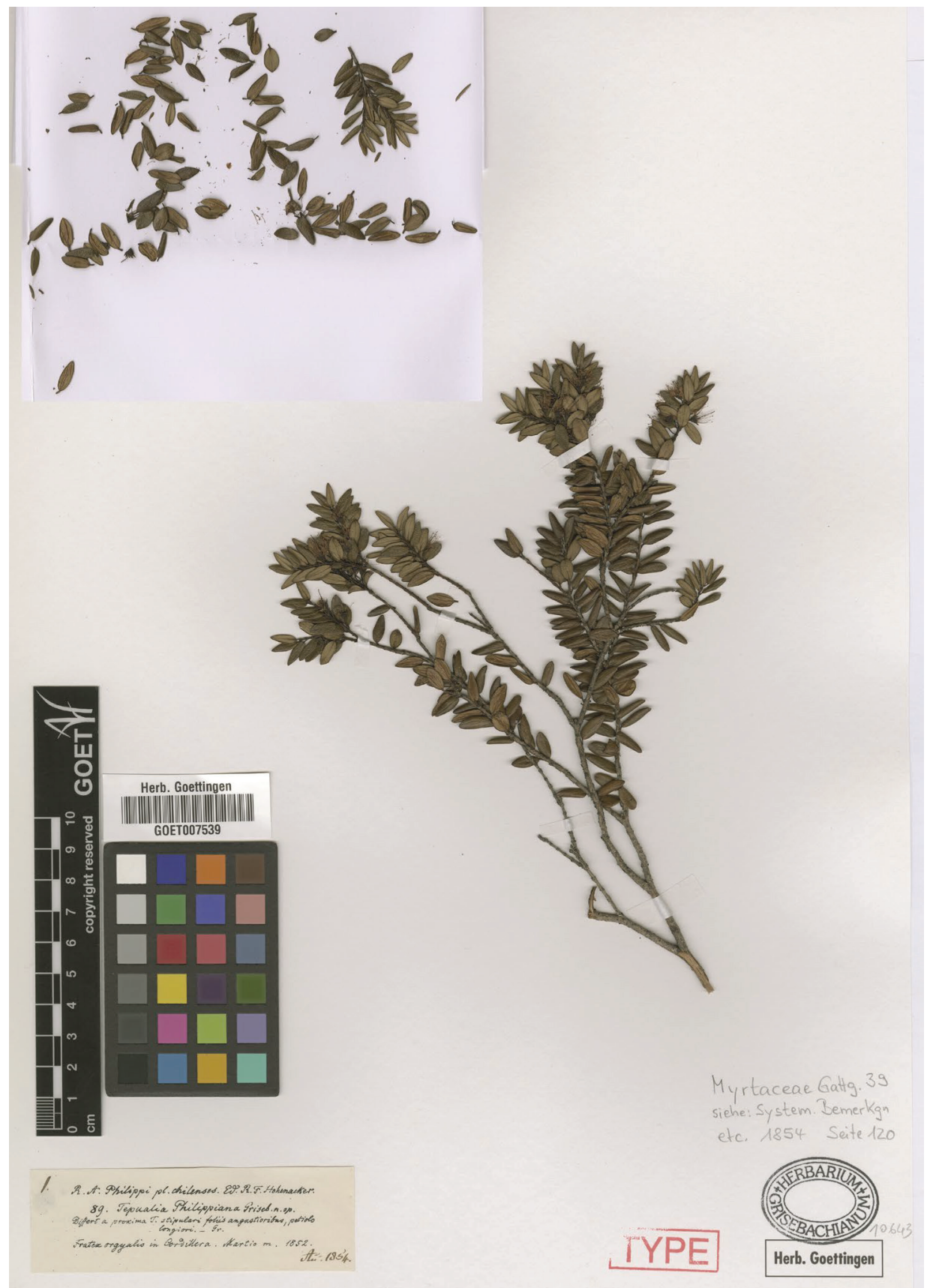

Abb. 12: Herbarbeleg von Tepualia stipularis (Hook. \& Arn.) Griseb., gesammelt von R. A. Pbilippi im Jahr 1852 in der chilenischen Provinz, Cordillera, Herbarium Göttingen, GOET007539. 


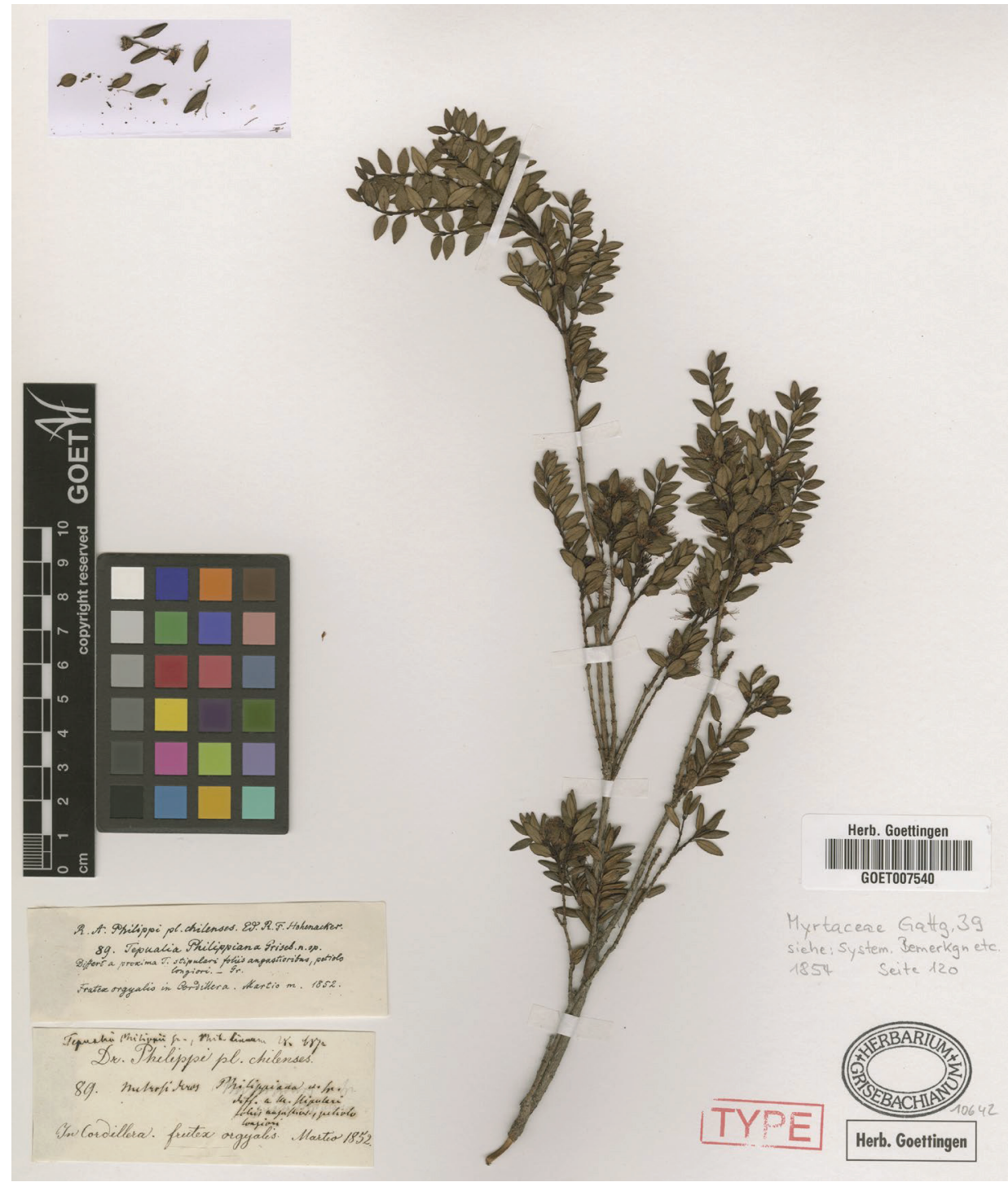

Abb. 13: Herbarbeleg von Tepualia stipularis (Hook. \& Arn.) Griseb., gesammelt von R. A. Philippi im Jahr 1852 in der chilenischen Provinz Cordillera, Herbarium Göttingen, GOET007540.

Die Herbarbelege der Pflanze Tepualia philippiana Griseb. (Tepualia stipularis (Hook. \& Arn.) Griseb.) unterscheiden sich deutlich von dem durch LantziusBeninga angefertigten Präparat. Während von letzterem bisher kein einziger Pflanzenbeleg in die Datenbank des Herbariums eingespeist worden ist, gehört Tepualia philippiana zu einer Vielzahl dort zu findender aus der Sammlung 
Grisebachs stammender Pflanzen. Dabei handelt es sich selbstverständlich nur um einen Bruchteil aller von ihm in der Sammlung befindlichen Belege. ${ }^{257}$ Dennoch werden hieran zwei Dinge deutlich: Zum einen verweist die hohe Anzahl von hinterlegten Digitalisaten auf eine hohe Typendichte in Grisebachs Herbarium. Zum anderen steht sie für ein erhöhtes Interesse an diesem Pflanzenmaterial und damit für eine verstärkte Bekanntheit, aber auch Bedeutsamkeit der wissenschaftlichen Arbeit Grisebachs bis in die Gegenwart. ${ }^{258}$

So weisen schon die roten Stempel die Belege von Tepualia philippiana als ,Type ${ }^{6}$ und damit als wertvolle Präparate aus. Wie der Großteil der digitalisierten Pflanzenexemplare von Grisebach wurden auch sie auf neuen Papierbögen fixiert. Die Etiketten machen deutlich, dass sie aber anders als beispielsweise Salicornia herbacea nicht selbst gesammelt wurden. Die Pflanzen stammen nicht von einer Exkursion Grisebachs, sondern wurden im März 1852 von Rudolph Amandus Philippi in der chilenischen Provinz Cordillera gesammelt und anschließend als Teil des Exsikkatenwerks ${ }^{259}$ R. A. Philippi pl. Chilenses Ed. R. F. Hohenacker des Pflanzensammlers Rudolf Friedrich Hohenacker (1798-1874) zum Verkauf angeboten. Laut Notiz auf einem der Etiketten wurden sie 1854 akquiriert. Der Akzessionskatalog sowie ein Schreiben Bartlings von 1866 machen deutlich, dass die Belege aber nicht - wie der Stempel ,Herbarium Grisebachianum` zunächst vermuten ließe - in Grisebachs Privatsammlung, sondern in das Universitätsherbarium übergingen. ${ }^{260}$ Als Teil der Universitätssammlung wurde Tepualia philippiana von Grisebach neu bestimmt, mit einem wissenschaftlichen Namen versehen und die Beschreibung in der Publikation Systematische Bemerkungen über die beiden ersten Pflanzensammlungen Philippi's und Lechler's im südlichen Chile und an der Maghellans-Straße auf Seite 32 mit direktem Verweis auf die Herbarbelege mit der Nummer 89 veröffentlicht. ${ }^{261}$

\footnotetext{
257 Im Jahr 1866, als Grisebach sein Herbar der Universität als Legat vermachte, umfasste seine Sammlung 40.000 Pflanzenarten. Das Universitäts-Herbarium zählte zu diesem Zeitpunkt 20.000 Exemplare. Vgl. UniA GÖ, Kur. Pers., 4973, Schreiben Grisebachs an das Universitätskuratorium, 18.12.1866; UniA GÖ, Kur. Pers., 4973, Schreiben des Universitätskurators an den königlichen Staatsminister und Minister der geistlichen Unterrichts- und Medizinalangelegenheiten Herrn Dr. von Mühler in Berlin, 1.7.1868.

258 Auf der Internetseite des Göttinger Herbariums wird das Grisebach-Herbarium auch tatsächlich als bedeutendster Teil der Sammlung gewertet. Vgl. Herbarium Göttingen.

259 Bei Exsikkatenwerken handelt es sich um Auflistungen von Pflanzenarten, die von Privatpersonen, Institutionen oder Tauschvereinen herausgegeben werden. Die aufgeführten Pflanzen, die als Dubletten vorliegen, werden auf Anfrage hin im Tausch gegen andere Exemplare verschickt, oder zum Kauf angeboten. Vgl. Wörz 2016, S. 22.

260 Vgl. UniA GÖ, Kur., 5592, Schreiben Bartlings an das Universitätskuratorium, 3.4.1866; PWI.Syst.Geobotan.HE2.

261 Vgl. Grisebach 1854, S. 32.
} 
Mit dem Zusammentragen von Pflanzenmaterial war nur ein erster Schritt zur Herausbildung eines Herbariums, wie es Bartling vorschwebte, getan:

\begin{abstract}
„Soll aber eine solche Sammlung das leisten, was von ihr verlangt werden kann, so kommt es nicht allein darauf an, daß sie möglichst viele verschiedene Arten in einzelnen Exemplaren vereinigt, sondern sie muss die einzelnen Arten in möglichster Vollständigkeit in ihren mannichfaltigen Formen von den verschiedensten Standtorten, und den verschiedenen Gegenden ihrer Verbreitungsbezirke, in den verschiedenen Entwicklungsstufen enthalten, sie muss so viel als möglich Original-Exemplare aus den Händen der Entdecker oder Beschreiber und von authentischen Standtorten nachweisen können, und zugleich in der Bezeichnung und Anordnung ihrer sämmtlichen Bestandtheile die Benutzung der neuesten wissenschaftlichen Untersuchungen und Ergebnisse darthun.“262
\end{abstract}

Indem die genaue Beschreibung und Bestimmung der Pflanzenexemplare als notwendige Voraussetzung für den Eingang von Präparaten in die Sammlung festgelegt worden war, ist das Göttinger Herbarium bereits in seinen Grundzügen als Forschungssammlung angelegt gewesen. Die vorausgehende Bearbeitung nahm dabei einen solchen Stellenwert ein, dass die Aufnahme neuen Pflanzenmaterials zu ihren Gunsten teilweise ausgesetzt werden musste. Wertigkeit erhielten die Objekte erst durch ihre korrekte Bezeichnung. Aus diesem Grund waren die Bestimmung und Etikettierung auch Aufgaben, die dem Vorsteher des Herbariums vorbehalten blieben. Diese Tätigkeiten durfte der Assistent - im Gegensatz zu den mechanischen Arbeiten - nur auf spezielle Anweisung hin vornehmen. ${ }^{263}$

Das Untersuchen der Pflanzen stellte dabei als Bestandteil des Zurichtungsprozesses von Herbarbelegen den Dreh- und Angelpunkt möglicher Forschungsarbeiten dar. Diese gingen dem Studium der Pflanze bereits voraus, wurden aber auch nach Fertigstellung des Belegs weitergeführt. Bereits das Einschätzen und Auswählen von Pflanzenmaterial, sowohl im Rahmen von Kaufhandlungen als auch im Tausch oder auf Reisen, kann als Teil des Beforschens verstanden werden. Die Erstellung der Belege nach dem von Bartling formulierten Anforderungsprofil legte dann allerdings die eigentliche Basis für den Auf- und Ausbau der Sammlung sowie für weiterführende botanische Forschungen. Für sie war ein gewissenhaft beschriebener und bestimmter Herbarbeleg notwendige Voraussetzung. ${ }^{264}$,Mit Herbarbelegen forschen` meint für das Göttinger

262 UniA GÖ, Kur., 5592, Schreiben Bartlings an das Universitätskuratorium, 21.3.1851.

${ }^{263}$ Die Passage in den Instruktionen lautet: , , [...] die Bestimmung und Etiquettierung der einzelnen Exemplare [ist] aber nur auf specielle Anweisung des Dirigenten vorzunehmen." UniA GÖ, Sek., 450.4, Instruktion für den Assistenten am königlichen Universitäts-Herbarium, 4.1.1853. Gleiches galt auch für den Tauschverkehr des Instituts: „Der Tauschverkehr selbst bleibt der Leitung des Dirigenten vorbehalten.“ UniA GÖ, Sek., 450.4, Instruktion für den Assistenten am königlichen Universitäts-Herbarium, 4.1.1853.

${ }^{264}$ Dass es sich bei der Bestimmung allerdings nicht um einen abgeschlossenen Prozess handelte, zeigt ein Auszug aus einem Schreiben Bartlings an das Kuratorium: ,[...] auch sind mehrere 
Herbarium deshalb zwei unterschiedliche Praktiken: Zur mit dem Herbarium verknüpften Forschungsarbeit gehörten zum einen die Bestimmung neu aufzunehmenden Pflanzenmaterials und zum anderen die darauf aufbauende Bearbeitung und weitere Untersuchung bereits fertiggestellter Belege. Sie wurde von den Göttinger Botaniker*innen sodann sehr unterschiedlich ausgestaltet. Dabei spielten sowohl die konkrete Anstellung und der damit verbundene Dienstrang als auch Tätigkeitsfelder und Verantwortlichkeiten sowie konkrete Forschungsinteressen eine zentrale Rolle.

Als Vorsteher des Herbariums widmete sich Bartling intensiv dem Ausbau der Sammlung. Das zeigt ihre vorübergehende Schließung und ihre Bearbeitung in den Räumlichkeiten in der Roten Straße, aber auch die später dem Kuratorium gegenüber wiederholt gemachten Aussagen zur Arbeit im Herbarium und deren Vorankommen. Die von ihm verfasste Vorschrift, als Vorsteher der Sammlung kein Privatherbar besitzen zu dürfen, verpflichtete Bartling dabei noch einmal mehr der Sammlung und trieb ihn in ihrem Ausbau an. Genauso wie die Forschungsreisen musste sich das Bearbeiten des Pflanzenmaterials im Herbarium dabei allerdings dem Hochschulkalender und dem Lehrbetrieb unterordnen. Letzterer war im Fach Botanik wiederum von den Jahreszeiten bestimmt. Schon die Arbeiten im botanischen Garten und dessen von Wetter und Jahreszeit abhängige Nutzung in Forschung und Lehre gaben den Jahresverlauf in mancher Hinsicht vor. Den Arbeiten im Herbarium konnte sich Bartling nur wenige Stunden wöchentlich und vorwiegend im Wintersemester widmen. ${ }^{265}$ Die Räumlichkeiten des Herbariums im ehemals Meisterschen Haus an der Paulinerstraße verfügten hierfür über zwei Arbeitstische. ${ }^{266}$ Darüber hinaus wurde es Studierenden und Dozierenden aber auch gestattet, Pflanzenexemplare

der wichtigsten und artenreichsten Pflanzenfamilien mit Benutzung der neuesten literarischen Hülfsmittel revidirt, und, wo es nöthig war, umgeordnet.“ UniA GÖ, Kur., 5592,

Schreiben Bartlings an das Universitätskuratorium, 16.11.1852.

265 So erklärt Bartling in einem Schreiben an das Kuratorium: „Mit der wissenschaftlichen

Bestimmung, der Einordnung und Catalogisirung des vorhandenen Materials habe ich in Gemeinschaft mit dem Dr. Lantzius-Beninga im vergangenen Winter mich anhaltend beschäftigt; im abgewichenen Sommersemester hat dagegen in dieser Beziehung nur wenig geschehen können, da es nothwendig gewesen war, sämmtliche [...] Landpflanzen im botanischen Garten anzuordnen und zu revidiren [...]." UniA GÖ, Kur., 5592, Schreiben Bartlings an das Universitätskuratorium, 14.11.1848. Auch an anderer Stelle wird die Beeinflussung der Forschungsarbeiten durch den Jahresverlauf deutlich: „Die erforderliche genaue Untersuchung vieler ausländischer, zum Theil bis jetzt noch unbeschriebener, Gewächse hat [...] eine raschere Zunahme der Sammlung fast unmöglich gemacht, da der ehrfurchtsvoll Unterzeichnete im Sommer nur durchschnittlich drei Nachmittage wöchentlich und an kurzen und dunkeln Tagen nur wenige Mittagsstunden auf dieselbe verwenden konnte [...]. “ UniA GÖ, Kur., 5592, Schreiben Bartlings an das Universitätskuratorium, 15.9.1835. Vgl. außerdem UniA GÖ, Kur., 5592, Schreiben Bartlings an das Universitätskuratorium, 29.7.1843; UniA GÖ, Kur., 5592, Schreiben Bartlings an das Universitätskuratorium, 26.2.1845.

266 Vgl. PWI.Syst.Geobotan.HV3; UniA GÖ, Kur., 5592, Rechnung über Einnahme \& Ausgabe für das Universitäts-Herbarium zu Göttingen vom 1. Juli 1851 bis dahin 1852, 16.11.1852. 
auszuleihen. ${ }^{267}$ Da sich das Herbarium von 1834 bis 1852 nicht in unmittelbarer Nähe des botanischen Gartens und der dort liegenden Dienstwohnung befand, ist davon auszugehen, dass Bartling diese Möglichkeit durchaus für seine Studien wahrnahm. Die Verlegung der Sammlung in den botanischen Garten sowie die nun durch Vorschriften abgesicherte Anstellung eines Assistenten wurden 1852 sodann auch als große Arbeitserleichterung angesehen:

„Durch die von Hohem Königlichen Universitäts-Curatorium gnädigst angeordnete Versetzung des Herbariums in ein im botanischen Garten belegenes Local ist manchen Uebelständen gründliche Abhülfe geworden, indem sowohl die Beaufsichtigung und Bearbeitung der Sammlungen bedeutend erleichtert, als auch die Benutzung desselben, namentlich in Bezug auf den botanischen Garten wesentlich gefördert ist." 268

Doch auch wenn Bartling dem Kuratorium gegenüber bereits 1835 betonte, dass die Sammlung trotz ihres geringen Umfangs „[... für die monographische Bearbeitung mancher Pflanzengruppen reiche Materialien darbietet" ${ }^{\text {" } 69}$, so fiel seine eigene Publikationstätigkeit eher bescheiden aus. Jene Werke, die auf seine Dissertation $^{270}$ von 1820 folgten, ihm zu seiner Anstellung an der Universität verhalfen und heute zu Bartlings Hauptwerken zählen - die mit Heinrich Ludolph Wendland (1791-1869) gemeinsam herausgegebenen Beiträge zur Botanike271 und die Ordines naturales plantarum ${ }^{272}$-, erschienen 1824/25 sowie 1830. Bei den nachfolgenden Veröffentlichungen handelte es sich, abgesehen von der gemeinsam mit Ernst Hampe herausgegebenen Arbeit über die Kryptogamen des Harzes, lediglich um kleinere Aufsätze. ${ }^{273}$ Diese weniger umfangreichen Arbeiten verdeutlichen allerdings, warum es an dieser Stelle eines differenzierteren Verständnisses von Forschungsarbeit bedarf. ${ }^{274}$ Es ist richtig, dass Bartlings Publikationstätigkeit mit Übernahme des Direktorats zurücktrat. ${ }^{275}$ Dass sich seine Haupttätigkeit auf den Ausbau der botanischen Anstalten verlegte, bedeutete aber keine generelle Abnahme seiner Forschungsarbeit. Seine Mitarbeit an den Plantae Ecklonianae 1832 sowie insbesondere die spätere Bearbeitung

\footnotetext{
267 Vgl. UniA GÖ, Sek., 450.4, Instruktion für den Assistenten am königlichen UniversitätsHerbarium, 4.1.1853.

268 UniA GÖ, Kur., 5592, Schreiben Bartlings an das Universitätskuratorium, 16.11.1852.

${ }^{269}$ UniA GÖ, Kur., 5592, Schreiben Bartlings an das Universitätskuratorium, 15.9.1835.

${ }^{270} \mathrm{Vgl}$. Bartling 1820.

271 Vgl. Bartling \& Wendland 1824; Bartling \& Wendland 1825. Zum Verhältnis von Bartling zu

H. L. Wendland vgl. Knoll 2005, S. 94, 96 u. 98.

272 Vgl. Bartling 1830.

273 Vgl. Drude 1875, S. 855; Wunschmann 1871b, S. 224.

${ }^{274}$ Im Vorwort des Sammelbandes From Private to Public stellt auch Marco Beretta fest: „Much more so than in other sciences, the role of writing in natural history is relatively secondary to the naturalist's techniques of handling and preserving specimens, by the care taken when organizing the species in a collection, and by the ways in which the exhibit is displayed to the public."

Beretta 2005, S. viii.

275 Vgl. Wunschmann 1971b, S. 224 f.
} 
einzelner Familien der Plantae Preissianae (1844-1847) bilden heute nur einen Ausschnitt seiner Arbeit ab - nämlich ausschließlich jenen Teil, der zur Veröffentlichung gebracht worden ist. ${ }^{276}$ Gerade die Bestimmung der von Preiß gesammelten Pflanzen verweist aber auf die an die Sammlungsführung gebundene Forschungstätigkeit. So ist davon auszugehen, dass die systematische Bearbeitung 277 im Rahmen dieser Publikation in Zusammenhang mit der 1843 von Preiß angekauften Sammlung stand. Genauso wie alle anderen neu in das Herbarium aufzunehmenden Pflanzenexemplare mussten auch sie zunächst bestimmt werden. Dass es in dem Zuge zu einer Veröffentlichung kam, war dann aber eine Ausnahme.

Mit Einrichtung der Sammlung 1832 widmete sich Bartling also intensiv der Pflanzenbestimmung. Damit arbeitete er weiterhin in seinem Forschungsgebiet, der Systematik, war dadurch zeitlich aber so eingeschränkt, dass eine Publikationstätigkeit nur noch bedingt möglich gewesen ist. Wie viel Raum die Bearbeitung der Sammlung getrockneter Pflanzen einnahm, zeigt sich auch in den Briefen, die Bartling an andere Botaniker*innen schickte. Im Austausch mit Kunze machte Bartling sein Vorankommen bezüglich der Poeppigschen Pflanzen ${ }^{278}$ wiederholt zum Thema und gegenüber Hampe äußerte er, nur noch wenig Zeit zu finden, um seinem persönlichen Interesse, den Kryptogamen, nachgehen zu können. ${ }^{279}$ Forschungsarbeit fand für Bartling also ganz im Namen des Instituts statt. Seine Fokussierung auf systematische Arbeiten, nach seiner explizit als dissertatio inauguralis geographico-botanica bezeichneten, also als dezidiert pflanzengeografisch ausgewiesenen, Dissertation, ist deshalb vor allem auch im Zusammenhang mit Bartlings Dienstpflichten zu betrachten. ${ }^{280}$

Ebenfalls aktiv in die (Forschungs-)Arbeiten des Herbariums eingebunden war der Assistent der Sammlung Lantzius-Beninga. Und auch dieser wandte sich nach seiner Dissertation ${ }^{281} 1844$ und einer weiteren Arbeit ${ }^{282}$ von 1847 nicht nur anderen thematischen Gebieten zu, sondern veröffentlichte auch deutlich weniger wissenschaftliche Texte. Diese Beobachtung konnte sich der Verfasser eines

\footnotetext{
276 Vgl. Drude 1875, S. 855; Wunschmann 1971b, S. 224.

277 Systematische Studien haben die Rekonstruktion der Phylogenie zum Ziel. Kernstück dieser Arbeiten sind ,ausführliche wissenschaftliche Bearbeitungen einer Gruppe (meist Gattung oder Familie), die alles bisherige Wissen zusammentragen, aber vor allem auf der eigenen Untersuchung möglichst aller Arten beruhen". Wagenitz 1993, S. 43 f.

278 Gemeint sind die im Rechnungsjahr 1839/40 durch Tausch gegen lebende Exemplare akquirierten von Eduard Friedrich Poeppig (1798-1868) in Peru und Brasilien gesammelten Pflanzen. Vgl. UniA GÖ, Kur., 5592, Schreiben Bartlings an das Universitätskuratorium, 14.12.1840.

279 Vgl. Briefe von F. G. Bartling an G. Kunze in der Universitätsbibliothek Leipzig; 2 Cod. Ms. philos. 182: F. G. Bartling, Brief von Friedrich Gottlieb Bartling an Ernst Hampe, 27.2.1848.

${ }^{280}$ Vgl. Wagenitz 1996, S. 11.

${ }^{281}$ Vgl. Lantzius-Beninga 1844.

282 Vgl. Lantzius-Beninga 1847.
} 
biografischen Artikels über Lantzius-Beninga augenscheinlich nicht erklären, wenn er schreibt,

„[...] eine Fortsetzung derselben [sei], wenn auch wohl geplant, doch nie zur Thatsache geworden und es [müsse] einigermaßen befremden, daß, nicht gar lange darauf, sich Lantzius-Beninga's Thätigkeit einem ganz anderen Gebiete botanischer Forschung zuwandte." $" 283$

Vor dem Hintergrund seiner Anstellung am Herbarium mag aber genauso wie auch bei Bartling weder die Abnahme seiner Publikationstätigkeiten noch die Fokussierung auf ausschließlich zwei systematische Arbeiten verwundern. ${ }^{284}$ Seit 1843 war Lantzius-Beninga Gehilfe in der Sammlung, promovierte 1844 und wurde 1846 habilitiert, in welchem Zuge ihm auch eine vorläufige Lehrberechtigung zugesprochen wurde. ${ }^{285}$ Dabei verfügte Lantzius-Beninga durchaus über die Möglichkeit, ein eigenes Herbar zu führen und, da er anders als der Vorsteher nicht vollkommen auf das Universitäts-Herbarium angewiesen war, theoretisch über einen weitaus größeren Handlungsspielraum. Doch auch wenn sein Verantwortungsbereich als weniger umfangreich zu bewerten ist und eine weitaus geringere Verpflichtung gegenüber der Sammlung bestand, muss die Einbindung in die Sammlungstätigkeiten umfassend gewesen sein.

Die von Lantzius-Beninga auf seiner im Sommer 1847 durchgeführten Forschungsreise nach Ostfriesland gesammelten Exemplare gingen beispielsweise genauso wie auch alle von Bartling auf Exkursionen zusammengetragenen Objekte direkt in die Sammlung über. ${ }^{286}$ Eine Entschädigung wurde dafür offenbar nicht entrichtet. Die auf diese Reise aufbauende und 1849 zur Veröffentlichung gebrachte Arbeit Beiträge zur Kenntniss der Flora Ostfrieslands ${ }^{287}$ ist dabei wie auch Bartlings wenige Publikationen im Rahmen der Tätigkeiten am Herbarium entstanden. ${ }^{288}$ Auch wenn Lantzius-Beninga gebürtiger Ostfriese war und damit

283 Wunschmann 1883, S. 702 f.

${ }^{284}$ Vgl. ebd.

285 Vgl. UniA GÖ, Kur. Pers., 5909, Schreiben der philosophischen Fakultät an das Universitätskuratorium, 5.9.1846.

286 „Der Zuwachs des Universitäts-Herbariums im Verlauf des Rechnungsjahrs 1847/48 hat [...] hauptsächlich in einer sehr reichen Sammlung ostfriesischer Gewächse, welche der Doctor Lantzius-Beninga eingeliefert, und in den von mir aus Südfrankreich zurückgebrachten Gegenständen bestanden. Beide Erwerbungen mussten für das Universitäts-Herbarium um so erwünschter seyn, je unvollständiger bis jetzt die Flora von Frankreich und die der norddeutschen Küstenländer in demselben repräsentirt war.“ UniA GÖ, Kur., 5592, Schreiben Bartlings an das Universitätskuratorium, 14.11.1848.

287 Vgl. Lantzius-Beninga 1847.

288 Bei der zweiten von Ernst Wunschmann benannten Veröffentlichung Lantzius-Beningas handelt es sich um eine Publikation mit dem Titel ,Die unterscheidenden Merkmale der deutschen Pflanzenfamilien und Geschlechter', die als Lehrbuch einzustufen ist. Diese erschien allerdings erst 1866 und fällt damit aus dem hier gewählten Untersuchungszeitraum.

Vgl. Wunschmann 1883. 
ein persönliches Interesse an dieser Region bestanden haben mag, gehörte die Flora des Königreichs Hannover doch zu den Schwerpunkten der Sammlung. Während die Idee zur Reise noch als weithin autarke Entscheidung zu werten ist, wurde die Publikation tatsächlich nicht von Lantzius-Beninga selbst, sondern durch das Kuratorium angeregt. ${ }^{289}$ Schon die Einrichtung einer regionalen Spezialsammlung, die neben der Hauptsammlung bestand, sprach für eine Sammlungstätigkeit im Sinne der Regierung. In welchem Maße man aber von Hannover aus kameralistische Absichten verfolgte, zeigt sich an dem Entstehungsprozess der Beiträge zur Kenntniss der Flora Ostfrieslands. Damit unterstreicht dieses Beispiel deutlich, wie sehr die Sammlung und damit auch die mit ihr einhergehende Forschung unter Einflussnahme des Königreichs und dessen Interessen standen.

Im Oktober 1848 sendete Lantzius-Beninga seinen Bericht über die Resultate seiner Forschungsreise an das Kuratorium. ${ }^{290}$ Aus ähnlichen Schriftwechseln nach den Reisen Bartlings geht hervor, dass die Reiseberichte üblicherweise lediglich zur Kenntnis genommen wurden und es in dem Zuge mitunter auch zur nochmaligen Bewilligung von Zulagen kam. Der Bericht Lantzius-Beningas wurde hingegen durch das Ministerium der geistlichen und Unterrichts-Angelegenheiten an das Königliche Ministerium des Inneren zur Einsicht weitergeleitet, da er Informationen über die Bodenverhältnisse und die Flora der Provinz Ostfriesland enthielt, „[...] welche vielleicht in landwirtschaftlichen Beziehungen für Königliches Ministerium des Innern einiges Interesse haben könnten [...]“291. Und mit dieser Einschätzung lag man offensichtlich richtig: Bereits am 25. Januar 1849 wurde ein Antwortschreiben verfasst mit dem Wunsch, den Aufsatz Lantzius-Beningas als Ergänzung der dem Göttinger Hofrat Georg Friedrich Wilhelm Meyer (1782-1856) aufgetragenen Ausarbeitung über die Flora des Königreichs Hannover (1842-1854) ${ }^{292}$ zu veröffentlichen. ${ }^{293}$ Als Vorlage für Lantzius-Beningas Ausarbeitung sollte die als Teil dieses mehrbändigen Werks 1836 erschienene Chloris Hanoverana ${ }^{294}$ dienen. Bereits das Titelblatt bietet einen sehr guten Eindruck darüber, was diesen Band, der eine kleinere abgeschlossene Flora bildete, auszeichnete, denn hier findet der Titel ,Chloris Hanoverana' durch einen Zusatz Erläuterung:

\footnotetext{
289 Vgl. UniA GÖ, Kur., 5835, Schreiben des Universitätskuratoriums an Lantzius-Beninga, 15.3.1849.

${ }^{290}$ Vgl. UniA GÖ, Kur. Pers., 5090, Schreiben Lantzius-Beningas an das Universitätskuratorium, 18.10.1848.

${ }^{291}$ UniA GÖ, Kur., 5835, Schreiben des Königlich-Hannoverschen Ministeriums der geistlichen und Unterrichts-Angelegenheiten (Braun) an Königliches Ministerium des Inneren, 15.1.1849.

292 Vgl. Wagenitz 2003, S. 46.

293 Vgl. UniA GÖ, Kur., 5835, Schreiben des Königlichen Ministeriums des Inneren an Königliches Ministerium der geistlichen und Unterrichts-Angelegenheiten, 25.1.1849.

294 Vgl. Meyer 1836.
} 
„Chloris Hanoverana oder nach den natürlichen Familien geordnete Übersicht der im Königreiche Hannover wildwachsenden sichtbar blühenden Gewächse und Farn nebst einer Zusammenstellung derselben nach ihrer Benutzung im Haushalte, in den landwirthschaftlichen Gewerben und in den Künsten.“295

Meyers Chloris Hanoverana ging demnach über eine übliche floristische Arbeit 296 hinaus, indem sie gleichermaßen einen Anwendungsanspruch verfolgte. Diese erklärende, auf die Nutzung angelegte Absicht schlug sich auch in der Aufmachung des Bandes nieder. Schon dem systematischen Teil wurde eine auszuklappende Karte als ,Erläuternde Übersicht der Anordnung vorgeschaltet, die die Verwandtschaftszugehörigkeiten übersichtlich darstellte. Jedem Verwandtschaftskreis ging dann eine Übersicht über die Gattungen voraus. Auffällig dabei ist, dass neben der wissenschaftlichen Bezeichnung stets auch der herkömmliche Name aufgeführt wurde. Zu jeder Art wurden zusätzlich immer Angaben zu der ersten Beschreibung, vorhandenen Abbildungen, Dauer, Blütezeit, Fruchtreife, Vorkommen und Standorten gemacht. Der letzte Teil des Bandes war ganz der Anwendung gewidmet, indem er die Einsatzmöglichkeiten der Gewächse fokussiert. ${ }^{297}$ Meyers Vereinszugehörigkeiten, zum Beispiel bei der Königlichen Landwirtschafts-Gesellschaft in Celle und der Naturhistorisch-ökonomischen Gesellschaft in Hannover, sowie der auf dem Titelblatt der Chloris Hanoverana in Anführungszeichen gesetzte Satz „Die Kenntnis des Vaterlandes gehört der Ehre der Nation an" 298 geben noch einmal mehr den Impetus dieses Bandes wieder. Denn als Physiograph des Königreichs Hannover stand Meyer mit seinem Werk ganz in der Tradition jenes Spannungsfelds zwischen Merkantilismus und Physiokratie, das, wie Hubert Steinke deutlich macht, seit dem 18. Jahrhundert neben Wissenschaft und Liebhaberei auch ein ökonomisches Interesse an den Pflanzen und der damit verbundenen Forschung hervorrief. ${ }^{299}$

\footnotetext{
${ }^{295}$ Ebd., Titelblatt.

${ }^{296}$ Die Floristik und Arealkunde untersucht den Artenbestand eines Gebietes oder die Verbreitung einzelner Arten. Die am Ende dieser Forschungen stehenden Florenwerke sind heute vor allem für Fragen des Artenschutzes bedeutsam. Vgl. Wagenitz 1993, S. 43 f.

297 Die Gewächse, die sich zum unmittelbaren Verbrauch oder zur Gewinnung roher Produkte des Pflanzenreichs eignen, teilte Meyer in Gewächse für den Haushalt, den Wiesen- und Weidenbau, die Viehhaltung, die Kultur der Heiden, die Befestigung des Treib- und Flugsandes und die Begrünung der Deiche, den Forstbau sowie den Gartenbau ein. Vgl. Meyer 1836, S. 691-711. Jene Gewächse, die sich zur Gewinnung von Kunstprodukten eignen, wurden in folgende Gruppen unterschieden: Gewächse für das Arznei- und Apothekerwesen, für das Manufaktur- und Fabrikwesen und für den Handwerksbetrieb. Vgl. Meyer 1836, S. 711-722.

${ }^{298}$ Ebd., Titelblatt.

${ }^{299}$ Vgl. Steinke 2008.
} 
Und an dieses konkrete Werk sollte die Publikation Lantzius-Beningas anknüpfen. Aus dem Austausch zwischen ihm und den beiden Ministerien geht hervor, dass man den Aufsatz in gleichem Format wie die bei Vandenhoeck und Ruprecht erschienene Chloris Hanoverana wünschte.300 Die Sicherstellung der Finanzierung durch das Ministerium des Inneren sowie die Lantzius-Beninga nach eigenem Ermessen zugestandene Überarbeitung täuschen dabei nicht über den deutlichen Aufforderungscharakter von Seiten des Kuratoriums hinweg:

\begin{abstract}
„Wir glauben voraussetzen zu dürfen, daß es den eigenen Wünschen des [...] Dr. Lantzius-
Beninga entsprechen werde, das Ergebnis seiner Forschungen in dieser Weise der Wissenschaft zugänglich zu machen, und werden es gern sehen, wenn derselbe Solches bewerkstelligt, wobei Wir es ihm, wie sich von selbst versteht, überlassen, dabei anstatt der gewählten Berichtsform nach seinem Ermessen auch eine andre ihm geeignet erscheinende Form für die Veröffentlichung zu gebrauchen“301,
\end{abstract}

heißt es in einem Schreiben des Kuratoriums vom März 1848. Dem Ansuchen folgend arbeitete Lantzius-Beninga den Aufsatz nach eigener Aussage im Sinne der Chloris Hanoverana um und gab 500 Exemplare in Auftrag. ${ }^{302}$

Dass das Ergebnis in seiner äußeren Aufmachung deutlich einfacher ausfiel, mag auch der politischen und finanziellen Situation geschuldet sein. Eine außerordentliche Ähnlichkeit ist allerdings nicht nur äußerlich sondern auch inhaltlich kaum festzustellen: Dem „Monumentalwerk““303 Meyers im Folioformat kommt der als Heft erschienene Aufsatz zumindest nicht nahe. ${ }^{304}$ Nach einer Einführung folgt auf eine ausführliche Darlegung der Bodenverhältnisse und einer Beschreibung der verschiedenen Floren ${ }^{305}$ Ostfrieslands ein systematisches Verzeichnis der Gefäßpflanzen samt ihrer Fundorte. Auch hier werden neben den wissenschaftlichen Namen die herkömmlichen Bezeichnungen genannt. Einen gezielt auf die Anwendung ausgerichteten Teil besitzt der Aufsatz jedoch nicht. Von besonderem Interesse ist an dieser Stelle allerdings die dem systematischen Verzeichnis angefügte Auflistung über die Anzahl der Arten je Familie, in der Lantzius-Beninga seine Ergebnisse denen der Chloris Hanoverana direkt gegenüberstellt. Deutlich tritt dadurch hervor, dass Lantzius-Beninga durch seine

300 Vgl. UniA GÖ, Kur., 5835, Schreiben des Ministeriums des Inneren an das Ministerium der geistlichen und Universitäts-Angelegenheiten, 7.3.1849.

301 UniA GÖ, Kur., 5835, Schreiben des Universitätskuratoriums an Lantzius-Beninga, 15.3.1849, Kopie.

302 Vgl. UniA GÖ, Kur., 5835, Schreiben Lantzius-Beningas an das Universitätskuratorium, 9.5.1849, Abschrift.

303 Wagenitz 1996, S. 11.

${ }^{304}$ Dies mag auch an den Veränderungen liegen, denen das botanische Publikationsformat insgesamt im Laufe des 19. Jahrhunderts unterlag. Vgl. Bonneuil 2002, S. 201. Für Untersuchungen, die gezielt Buchpublikationen in den Blick nehmen, vgl. Ford 2014; Yale 2016.

${ }^{305}$ Lantzius-Beninga unterscheidet zwischen sieben Floren: Flora der kultivierten Geest, Flora des Waldbodens, Flora der wilden Heide (der Heide im engeren Sinne), Flora der Meeden oder des Gebiets der natürlichen Wiesen, Flora des Moors, Flora der Marsch und Flora der Inseln. 
Untersuchungen den 311 von Meyer benannten Arten insgesamt 403 neue hinzufügen sowie neue Standorte für die bereits benannten Arten ausmachen konnte. ${ }^{306} \mathrm{Ob}$ diese Gegenüberstellung aus rein wissenschaftlichem Interesse erfolgte oder auch aus einem Konkurrenzdenken heraus gewählt wurde, lässt sich aus heutiger Perspektive nicht rekonstruieren. Die auf diese Aufstellung folgende zweiseitige Zusammenfassung setzte allerdings einen ganz anderen Fokus als Meyers Veröffentlichung: Auf Grundlage des Auszählens der Arten und des Zusammentragens der Standorte traf Lantzius-Beninga Aussagen über das Vorkommen der Pflanzen. Hervorgehoben wurden von ihm jene Arten, die neu oder von besonderem Interesse für die Flora des gesamten Königreichs Hannover sowie für die Flora Deutschlands seien. ${ }^{307}$ Damit verzichtete Lantzius-Beninga also nicht allein auf einen anwendungsbezogenen Teil, sondern platzierte einen von der Chloris Hanoverana abweichenden Inhalt, der nicht auf Anweisung des Kuratoriums hin, sondern offenbar aus eigenem Interesse entstanden war. Das Ministerium selbst schien mit dem Ergebnis dennoch zufrieden. Von den 75 Exemplaren, die Lantzius-Beninga dem Kuratorium zur weiteren Nutzung zusandte, leitete das Ministerium des Inneren, ein Schreiben vom 25. Mai 1849 an sämtliche Landdrosteien und die Berghauptmannschaft zu Clausthal begleitend, einige Ausfertigungen

„zum eigenen Gebrauche, beziehungsweise zur angemessenen Vertheilung im Verwaltungs-

bezirke, wobei die landwirthschaftlichen Vereine [...] zu berücksichtigen seyn werden“"308,

weiter. ${ }^{309}$

In seiner Form kleiner und handlicher als Meyers Chloris Hanoverana war der Aufsatz Lantzius-Beningas mit Sicherheit leicht in Umlauf zu bringen. Und auch wenn nicht bekannt ist, ob und in welche landwirtschaftlichen Vereine sie gelangt ist und dort auch gelesen wurde, so zeigt sich am Beispiel dieser Publikation deutlich die Verstrickung wissenschaftlichen Arbeitens mit ökonomischen und politischen Interessen. Wenn Wagenitz in Bezug auf Meyers Flora des Königreichs Hannover kommentiert, „[e]s bedürfte eines speziellen Studiums um herauszufinden, was daran originell ist ${ }^{\text {"310 }}$, dann urteilt er aus einer wissenschaftlichen Anspruchshaltung heraus. Sowohl Meyers groß angelegtes Werk als auch Lantzius-Beningas Aufsatz gingen aber in ihrem Anspruch über einen wissenschaftlichen Nutzen hinaus. Denn wie sie auch rezipiert worden sein

\footnotetext{
306 Wunschmann stuft Lantzius-Beningas Arbeit als durchaus bedeutend für die Kenntnis der Flora Ostfrieslands ein: „Man kann sagen, daß durch dieses Werk die Flora eines bis dahin zum größten Theil botanisch noch unbekannten Gebietes in ihren Hauptzügen festgestellt worden ist." Wunschmann 1883, S. 702-703.

307 Vgl. Lantzius-Beninga 1849, S. 54 f.

308 UniA GÖ, Kur., 5835, Schreiben des Ministeriums des Inneren an sämtliche Landdrosteien und die Berghauptmannschaft zu Clausthal, 25.5.1849.

309 In der Akte ,UniA GÖ, Kur., 5835` sind noch acht Exemplare enthalten.

310 Wagenitz 1996, S. 11.
} 
mögen, so stand hinter ihrer Publikation die Idee der Verwertbarkeit von Forschungsergebnissen. Die mögliche Weiterleitung des Aufsatzes über die Flora Ostfrieslands Lantzius-Beningas an landwirtschaftliche Vereine stand dabei sowohl in der Tradition kameralistischen Denkens als auch eines aufklärerischen Bildungsanspruchs. ${ }^{311}$

Gerade in Bezug auf die Arbeiten von Bartling und Lantzius-Beninga tritt deutlich hervor, wie die Regierung auf die wissenschaftliche Forschungsarbeit Einfluss nahm. Nicht nur begünstigte sie Arbeiten, die den Interessen des Königreichs dienten. Die Etablierung und der Ausbau eines an den wissenschaftlichen Standards der Zeit orientierten Herbariums konnten nur durch Unterstützung des Kuratoriums möglich gemacht werden. Die Verpflichtung der Universität und dem Kuratorium gegenüber band Bartling und Lantzius-Beninga massiv an die Sammlung. Gerade für den Direktor des Instituts bedeutete seine Stellung in der Folge mitnichten eine unabhängige, frei bestimmte und vornehmlich den persönlichen Interessen folgende Forschungstätigkeit.

Das wissenschaftliche Arbeiten mit Herbarbelegen der neben Bartling und Lantzius-Beninga als Professoren am Göttinger Institut tätigen Botaniker*innen gestaltete sich im Vergleich dazu anders. Während der noch bis 1836 wirkende Schrader die Arbeit am Herbarium allerdings nicht unterstützte, sondern ausschließlich mit seinem Privatherbar arbeitete, war Grisebach der Sammlung sehr geneigt. Genauso wie Schrader arbeitete er zwar mit einer Privatsammlung, doch besaß er eine vollkommen andere Haltung zu der Bedeutsamkeit des Herbariums als Institution. Dass er der Sammlung unterstützend gegenüberstand, drückt sich nicht zuletzt in einem Ansuchen vom 19. Juni 1846 ab. ${ }^{312}$ In diesem formulierte Grisebach den Wunsch, Bartling bei der Verwaltung des Universitätsherbariums assistieren zu dürfen, dem in der Folge auch stattgegeben wurde. ${ }^{313}$ Die Direktion und Rechnungsführung blieben Bartling weiterhin vorbehalten, doch wurde Grisebach bezüglich der Anschaffung von Materialien und der Einrichtung der Sammlung eine entscheidende Stimme zugesprochen. Gleichzeitig wurde ihm im Gegenzug zu seinem Mitwirken in der Verwaltung und bei der

311 So arbeitet Marten Pelzer die Verknüpfung von Bildung und Lesen als primäres Ziel landwirtschaftlicher Vereine am Beispiel des landwirtschaftlichen Vereinswesens des ehemaligen Fürstentums Lüneburg im 19. Jahrhundert deutlich heraus. Dabei zeigt er, dass bei der für die Vereinsbibliothek ausgewählten Literatur vor allem der praktische Nutzen der Schriften im Vordergrund stand. Zu den Autor*innen der Zeitschriften und landwirtschaftlichen Bücher, die von populären, möglichst kurz gefassten bis hin zu wissenschaftlich gehaltenen Werken reichten, gehörten neben Agrarschriftsteller*innen, Geistlichen, populären Vielschreiber*innen und Vertreter*innen des landwirtschaftlichen Vereinswesens auch Professor*innen und prominente Landwirtschaftslehrer*innen. Vgl. Pelzer 2004. Für eine erste umfassende Arbeit zum landwirtschaftlichen Vereinswesen des 19. und frühen 20. Jahrhunderts siehe Marten Pelzers Studie zum Landwirtschaftlich-gewerblichen Verein zu Badbergen. Vgl. Pelzer 2002. 312 Vgl. UniA GÖ, Kur., 5584, Schreiben Grisebachs an den Geheimen Kabinettsrat, 19.6.1846.

313 Vgl. UniA GÖ, Kur., 5584, Schreiben des Universitätskuratoriums an Bartling und Grisebach, 20.7.1846. 
Bearbeitung des Pflanzenmaterials die Möglichkeit eingeräumt, die Sammlung jeder Zeit nutzen zu dürfen. Und gerade dieser Punkt schien in Hinblick auf Grisebachs Forschungsinteressen ausschlaggebend für sein Gesuch gewesen zu sein.

Denn auch Grisebach widmete sich intensiv der Taxonomie und machte sich schon zu Studienzeiten als Systematiker einen Ruf. ${ }^{314}$ Sein privates Herbarium war der zentrale Ausgangspunkt seiner Forschung. ${ }^{315}$ Deren Ziel bestand jedoch nicht allein darin, einen Beitrag zur Systematik zu leisten. Vielmehr bildeten Grisebachs systematische Studien die Grundlage für seine pflanzengeografischen Überlegungen, die 1872 in Die Vegetation der Erde nach ibrer klimatischen Anordnung 316 mündeten. ${ }^{317} \mathrm{Um}$ ein Verständnis von dem Gesamtbild der Vegetation und der Verbreitung der Pflanzengruppen auf der Erde zu erlangen, war nicht nur eine umfassende Lektüre, sondern auch das Studium der Herbarien verschiedener Länder notwendig. Deshalb führte Grisebach nicht allein Forschungsreisen durch, sondern ergänzte seine Sammlung ebenso durch Ankäufe und den Tausch von Pflanzenmaterial. In seinem Herbarium überstieg die Anzahl der Exemplare aus diesem Grund häufig jene der Arten. 318 Doch auch wenn Grisebachs Privatsammlung sehr umfangreich gewesen ist, so war er zur Erlangung eines Überblicks über sämtliche Floren bestrebt, das Göttinger Universitätsherbarium ergänzend für seine Arbeiten hinzuzuziehen. In seinem Schreiben hob er besonders das Anliegen hervor, möglichst uneingeschränkt im Universitätsherbarium arbeiten zu können, und schien vor allem aus diesem Grund Bartling assistierend zur Seite gestellt werden zu wollen. ${ }^{319}$

Der Beleg der Pflanze Tepualia philippiana zeugt auch tatsächlich von einer solchen Nutzung der Institutssammlung und eines sich gegenseitigen Befruchtens zwischen Herbarium und Grisebachs Forschungsarbeit: Das Präparat gelangte als Teil eines größeren käuflich erworbenen Pflanzenkonvoluts des nach Chile emigrierten Naturforschers Philippi in die Göttinger Sammlung. ${ }^{320}$ Die Publikation Systematische Bemerkungen über die beiden ersten Pflanzensammlungen Philippi's und Lechler's im südlichen Chile und an der Maghellans-Straße ${ }^{321}$ von 1854 macht offenkundig, dass Grisebach sich intensiv mit diesen Pflanzen beschäftigt hatte. Der

\footnotetext{
314 Vgl. Grisebach 1880, S. 606-608.

315 Vgl. ebd., S. 626 f.; Mägdefrau 2013, S. 128.

316 Vgl. Grisebach 1872a; Grisebach 1872b.

317 Vgl. Mägdefrau 2013, S. 128.

318 Vgl. Grisebach 1880, S. 626.

319 „Dagegen ist mir, um im Lokale des Universitäts-Herbarii jederzeit arbeiten zu können, daran gelegen, in der Direction dieses letztern Instituts Herrn Professor Bartling adjungirt zu werden und namentlich zur Führung eines Schlüssels zu dessen Räumen berechtigt zu sein.“ UniA GÖ, Kur., 5584, Schreiben Grisebachs an den Geheimen Kabinettsrat, 19.6.1846.

320 Vgl. UniA GÖ, Kur., 5592, Schreiben Bartlings an das Universitätskuratorium, 3.4.1866; PWI.Syst.Geobotan.HE2., Vorläufige Übersicht der Einnahmen und Ausgaben 1. Juli 1853 bis dahin 1864.

321 Vgl. Grisebach 1854.
} 
Kaufpreis und genaue Ablauf der Akquirierung lassen sich zwar nicht mehr rekonstruieren, doch die expliziten Verweise auf das Herbarmaterial machen offenkundig, dass Grisebach nicht auf der Grundlage seiner Privatsammlung arbeitete. Dass seine systematischen Studien und die Auseinandersetzung mit der chilenischen Flora auch hier Teil größer angelegter Fragestellungen gewesen sind, zeigt sich dann im zweiten Band der Vegetation der Erde nach ibrer klimatischen Anordnung, in dem Grisebach dem chilenischen Übergangsgebiet ein eigenes Kapitel widmete. ${ }^{322}$

Neben der Fokussierung pflanzengeografischer Betrachtungen unterschied sich Grisebachs Forschung von der Bartlings und Lantzius-Beningas dadurch, dass sie in zahlreichen Publikationen Ausdruck fand. ${ }^{323}$ Daraus kann geschlossen werden, dass Grisebach, obwohl ihm Bartling als Direktor übergeordnet gewesen ist, eine deutlich größere wissenschaftliche Reichweite besaß. Der untergeordnete Status am Institut war mit Blick auf Grisebachs Wirken schnell nicht mehr angemessen, sodass er 1847 - im gleichen Jahr, in dem er die Mitwirkung am Herbarium zugesprochen bekam - auch vom außerordentlichen zum ordentlichen Professor in der Hierarchie aufstieg. ${ }^{324}$ Damit gab es erstmals zwei ordentliche Professuren im Fach Botanik gleichzeitig. ${ }^{325}$ Grisebachs wissenschaftliche Arbeit wurde durch ihre Veröffentlichung aber nicht allein publik gemacht, sondern die Publikationen selbst waren ein Spiegelbild seiner Vernetzung. Schon in Bezug auf Bartlings Forschungstätigkeit wurde deutlich, dass diese genauso wie die Akquirierung des Materials selbst nicht in Abgeschiedenheit stattfand, sondern im Austausch mit anderen Institutionen und Kolleg*innen: So bearbeitete er beispielsweise Pflanzen des Berliner Herbariums ${ }^{326}$, holte die Einschätzung von anderen Wissenschaftler*innen ein $^{327}$ und veröffentlichte gemeinsam mit anderen Botaniker*innen. Grisebachs Wirken überstieg Bartlings Ausmaß wissenschaftlicher Vernetzung aber bei Weitem. Grund dafür waren nicht nur seine umfangreicheren Forschungsreisen. ${ }^{328}$ Vielmehr machte sein pflanzengeografischer Anspruch eine umfassendere Vernetzung notwendig. Grisebach arbeitete zu

\footnotetext{
322 Vgl. Grisebach 1872, S. 467-480.

323 Vgl. Grisebach 1880, S. 606-620.

324 Vgl. Grisebach 1880, S. 620.

325 Während Bartling aber der philosophischen Fakultät angehörte, war Grisebachs Professur an der medizinischen Fakultät angesiedelt.

326 Vgl. 2 Cod. Ms. philos. 182: F. G. Bartling, Brief von Friedrich Gottlieb Bartling an Ernst Hampe, 27.2.1848.

327 In einem Brief an Ernst Hampe unterbreitete Bartling ihm folgenden Vorschlag: „Es würde mich sehr freuen, wenn Sie sich entschlössen, beim Beginn der schönen Jahreszeit einige Tage bei mir zuzubringen: wir könnten dann zusammen unter anderm auch die Ausbeute von meiner letzten Reise, die bis dahin nur zum kleinsten Theile eingeordnet wird, durchgehen - einiges fände sich dann auch wohl noch für Ihre Sammlung. “ 2 Cod. Ms. philos. 182: F. G. Bartling, Brief von Friedrich Gottlieb Bartling an Ernst Hampe, 27.2.1848.

328 Grisebach bereiste unter anderem den Balkan (1839/40), Norwegen (1842), die Pyrenäen (1850) sowie die Karpaten (1852). Vgl. Grisebach 1880, S. 607-613.
} 
einzelnen Pflanzengruppen, verfasste aber auch floristische Arbeiten. Der darauf aufbauende Blick auf die Verbreitung der Arten machte es erforderlich, Exemplare aus allen Ländern, in denen diese vorkommen, mit in die Betrachtungen einzuschließen. ${ }^{329}$ So zeugt der 1847 veröffentlichte Aufsatz Über die Vegetationslinien des nordwestlichen Deutschlands ${ }^{330}$ davon, dass Grisebach sich mit einer ähnlichen Region wie Lantzius-Beninga befasste. Doch Lantzius-Beninga verfasste ein Florenwerk, das auf den Ergebnissen seiner eigens durchgeführten Forschungsreise entstand. Grisebach hingegen arbeitete klimatische Vegetationslinien sowie engere Vegetationsbezirke heraus. Sehr deutlich tritt dabei hervor, wie die Publikationen und Ergebnisse anderer seine pflanzengeografischen Arbeiten bedingten. Das von ihm angelegte, Verzeichniss der Pflanzen, welche im nordwestlichen Deutschland eine ihrer Vegetationslinien erreichen' gründete sich nicht allein auf eigene Untersuchungen. Vielmehr verweist Grisebach auf Florenwerke anderer Wissenschaftler*innen, schriftliche Mitteilungen sowie auf mündliche Aussagen seiner Göttinger Kollegen Bartling und Lantzius-Beninga. 331 Die Ergebnisse der Ostfriesland-Exkursion werden hier von Grisebach fruchtbar gemacht. Von Interesse war dabei weniger das Vorkommen einer für die Region typischen Pflanze wie Salicomia herbacea, stattdessen griff Grisebach eine Pflanze aus dem Ergebnisteil der zwei Jahre später erscheinenden Beiträge zur Kenntniss der Flora Ostfrieslands auf. Primula acaulis Jacq. (Primula vulgaris Hudson ${ }^{332}$ ) wird dort

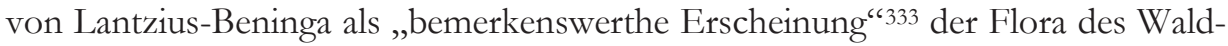
bodens Ostfrieslands und als „neи [Hervorhebung im Original] für das Königreich“"334 eingestuft. Für Grisebach wird sie damit zu einer von 81 Pflanzen, die die östlichen Vegetationslinien markieren. ${ }^{335}$

Die Unterscheidung zweier Formen des Forschens mit den Sammlungsobjekten des Herbariums - die Bestimmung der in die Sammlung zu integrierenden Pflanzenpräparate sowie das daran anschließende Beforschen der fertiggestellten Belege - schärft den Blick dafür, dass die wissenschaftliche Arbeit bereits in den Belegen selbst steckt. Denn das Fehlen umfangreicher Bibliografien täuscht darüber hinweg, dass schon das Forschen mit Herbarbelegen bei ihrer Erstellung beginnt und diese einen zeitintensiven Bestandteil der Arbeit mancher Dozierender darstellte. Bartling und Lantzius-Beninga sind mit der Bearbeitung der Sammlung stark ausgelastet gewesen, was nicht zuletzt auch durch die Tatsache unterstrichen wird, dass einige Konvolute noch Jahrzehnte nach ihrer Akquirierung nicht bearbeitet worden waren. Im großen Rahmen betrachtet schlug sich ihre systematische Arbeit allerdings in der Bezeichnung der Belege und

\footnotetext{
329 Vgl. Mägdefrau 2013, S. 128.

330 Vgl. Grisebach 1880.

331 Vgl. Grisebach 1880, S. 158-191.

332 Synonym genutzte Namen sind außerdem Primula acaulis (L.) Hill und Primula veris var. acaulis L.

333 Lantzius-Beninga 1849, S. 14.

334 Ebd., S. 54.

335 Vgl. Grisebach 1880, S. 173.
} 
damit in einem kontinuierlichen Anwachsen der Sammlung nieder: Orientiert an Bartlings Ordines naturales plantarum wurden die Pflanzenexemplare bestimmt und im Anschluss daran aufgestellt. Am Ende dieses Forschens standen nach einem einheitlichen System bezeichnete, auf Papierbögen fixierte Pflanzenbelege, abgelegt in einem Schrank. Nur vereinzelt führte diese Arbeit zu einer Publikation. Das Bestimmen der Pflanzenexemplare bildete darüber hinaus jedoch nicht nur die Grundlage für das Entstehen des Herbariums selbst, sondern stellte die Voraussetzung für weiterführende Forschungstätigkeiten dar. Schon in seinem 1875 verfassten Nachruf stellte Drude fest, dass Bartling dem Institut mit der Gründung des Herbariums „,...] eine wichtige Quelle für systematischpflanzengeografische Studien geschaffen " 336 habe. Grisebachs zahlreichen Arbeiten zur Pflanzengeografie, wenn auch nicht allein auf Grundlage des Universitätsherbariums entstanden, zeugen schließlich von dem aktiven Nutzen dieser Quelle.

Das aufeinander aufbauende Arbeiten der Botaniker*innen am Göttinger Herbarium illustriert beispielhaft jenen Wandel des ontologischen Status der Arten, den Müller-Wille für die botanische Forschung seit der Begründung Linnés binärer Nomenklatur festmacht. ${ }^{337}$ Denn durch die Etablierung dieses Systems und des daran gebundenen Regelwerks verloren die wissenschaftlichen Namen ihre Beschreibungsfunktion und besaßen hingegen ausschließlich eine Bezeichnungsfunktion. Damit wurden die Arten über sprachliche und räumliche Grenzen hinweg vergleichbar und der Informationsaustausch begünstigt. ${ }^{338}$ Eine gemeinsame Grundlage in der Bestimmung der Arten war die Voraussetzung für die Weiterentwicklung botanischer Forschungsarbeit und die Etablierung neuer Forschungsperspektiven. Die unterschiedliche thematische Fokussierung der am Göttinger Institut tätigen Botaniker*innen bildet deshalb auch eine generelle Verschiebung in der botanischen Forschung ab. Von der Beschreibung und Katalogisierung entwickelte sie sich hin zu Fragestellungen, die auf die regionale und globale Verbreitung der Pflanzen abzielten.339 Grisebachs Arbeiten zur Pflanzengeografie wären ohne die weltweite Verwendung der binären Nomenklatur also nicht möglich gewesen:

\footnotetext{
336 Drude 1875, S. 855.

337 Vgl. Müller-Wille 2017b, S. 126.

338 Schon seit der Herausbildung der binären Nomenklatur ist die Botanik als, wordy science $z u$ verstehen. Vgl. Daston 2001, S. 270. Doch erst eine erhöhte begriffliche Standardisierung zur Vermeidung von Synonymen in der Mitte des 19. Jahrhunderts führte zur Herausbildung einer gemeinsamen wissenschaftlichen Sprache. Vgl. Bonneuil 2002; Daston 2004, S. 153-158.

Diese Entwicklung der Botanik hin zu umfassenden und verbindlichen Konzepten lässt sich besonders durch den von Daston und Galison geprägten Begriff der ,communitarian objectivity ${ }^{6}$ fassen. Vgl. Daston 1999, S. 85-91; Daston 2001.

339 Vgl. Bonneuil 2002, S. 195; Müller-Wille 2017b, S. 119 f.
} 
„The shares that certain plant families held in the overall number of genera and species of a certain climate or region played a fundamental role in the attempts of Augustin de Candolle, Alexander von Humboldt, and Robert Brown to establish ,laws' that governed the geographic distribution of plants in the second and third decades of the nineteenth century.“340

Indem die Standardisierung in der Bezeichnung sich zum Paradigma ${ }^{341}$ erhob, erhielten die Artnamen folglich neue empirische Bedeutung. Sie wurden zu

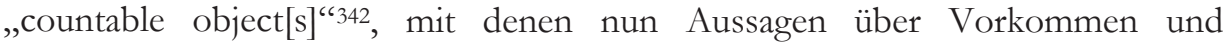
Verbreitung von Pflanzen gemacht werden konnten. ${ }^{343}$ In Kombination mit zusätzlichem Datenmaterial wurden auf Grundlage von Herbarbelegen nun neue Forschungsfelder bestritten. ${ }^{344}$

Grisebach bespielte demnach eine andere und neue wissenschaftliche Bühne als die ihm vorausgehenden Göttinger Professor*innen. ${ }^{345}$ Seine Forschungsarbeit knüpfte an die sich verschiebende Rolle der Systematik an, während sich Bartling und Lantzius-Beninga vornehmlich auf die Vervollständigung der Sammlung fokussierten. Kooperation in der wissenschaftlichen Arbeit wurde von Grisebach darüber hinaus in einem weitaus größeren Rahmen betrieben. 346 Dass sein Bekanntheitsgrad und sein Ansehen aus gegenwärtiger Perspektive als sehr viel höher zu bewerten sind, ist deshalb nicht allein durch die Etablierung neuer Forschungsperspektiven zu erklären, sondern auch auf seine Vernetzung und hohe Publikationsdichte zurückzuführen. Denn offenkundig wurde wissenschaftliche Produktivität bereits im zweiten Viertel des 19. Jahrhunderts maßgeblich an den Veröffentlichungen bemessen. Wiederholt informierten die Göttinger Botaniker*innen das Kuratorium über ihr Vorankommen in Projekten und legten ihren Schreiben Publikationslisten oder gar Veröffentlichungen selbst bei. ${ }^{347}$

\footnotetext{
340 Müller-Wille 2017b, S. 125.

341 Eine offizielle Richtlinie stellte der von Alphonse de Candolle (1806-1893) 1867 in Vorschlag gebrachte Internationale Code der Botanischen Nomenklatur (seit 2011 Internationaler Code der Nomenklatur für Algen, Pilze und Pflanzen) dar. Vgl. Daston 2004.

342 Müller-Wille 2017b, S. 126.

$343 \mathrm{Vgl}$. Stafleu 1987, S. 165.

344 Vgl. Bourguet 2002; Bourguet 2005.

345 Vgl. Wagenitz 2003, S. 46.

346 Nennenswert ist beispielsweise die Zusammenarbeit Grisebachs mit William Hooker (1785-1865) und den Royal Botanic Gardens (Kew). Im Auftrag der britischen Regierung reiste er mehrmals nach London, um Pflanzenmaterial der ,westindischen Flora' zu bearbeiten und veröffentlichte schließlich eine mehrbändige Flora of the British West Indian Islands (1859-1864). Vgl. Bonneuil 2002, S. 201; Grisebach 1880, S. 614.

347 Vgl. UniA GÖ, Kur. Pers., 5828, Schreiben Bartlings an den Kanzleirat, 2.4.1836; UniA GÖ, Kur. Pers., 5828, Schreiben Bergmanns an das Universitätskuratorium, 22.5.1836; UniA GÖ, Kur. Pers., 4973, Schreiben Grisebachs an das Universitätskuratorium, 17.5.1852; UniA GÖ, Kur. Pers., 4973, Schreiben Grisebachs an das Universitätskuratorium, 1854.
} 
Forschungsthemen, Publikationstätigkeit und Grad der Vernetzung bilden deutlich ab, in welchem Spannungsverhältnis sich das Göttinger Institut und mit ihm auch die dort tätigen Wissenschaftler*innen im Zeitraum von 1832 bis 1852 befanden. Tätigkeiten bauten aufeinander auf, während sich Forschungsschwerpunkte gleichzeitig massiv verschoben. Die Stellung am Institut brachte dabei auf der einen Seite Entscheidungsgewalt, bedeutete auf der anderen Seite jedoch auch eine erhöhte Rechenschaft gegenüber dem Kuratorium sowie im Falle Bartlings eine starke Bindung an die Sammlungen. Wissenschaftliche Arbeiten wie beispielsweise Lantzius-Beningas Beiträge zur Flora Ostfrieslands bilden dabei deutlich ab, inwieweit die Ansprüche der Regierung mit den eigenen wissenschaftlichen Interessen verhandelt werden mussten. Der vereinnahmende Ausbau der Sammlung erschwerte es dabei den Verantwortlichen, ein freieres und innovativeres Arbeiten zu verfolgen. 


\subsection{Mit Herbarbelegen lehren}

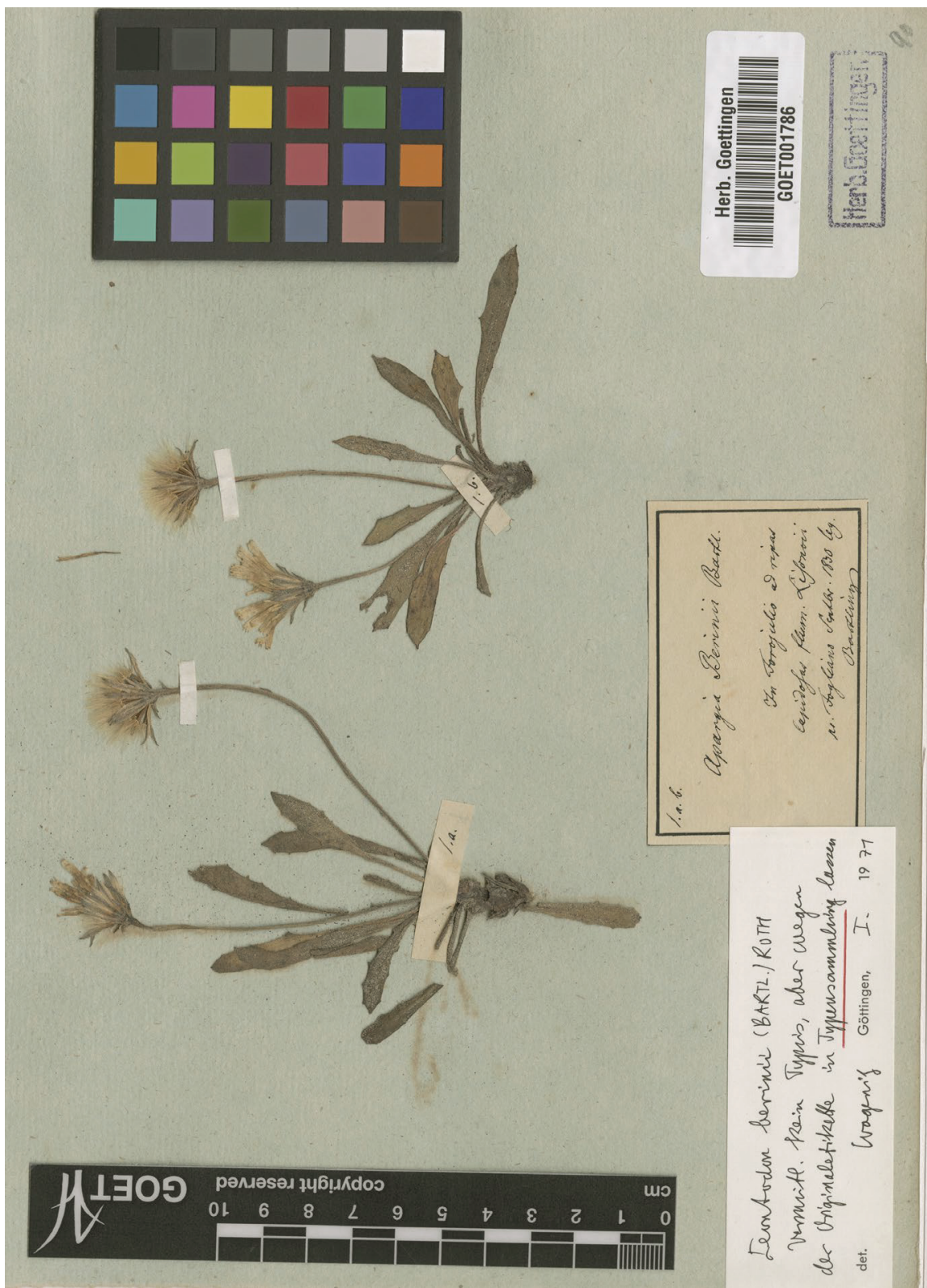

Abb. 14: Herbarbeleg von Leontodon berinii (Bartl.) Roth, gesammelt von F. G. Bartling im September 1830 in Forojulio in der Nähe des italienischen Ortes Fogliano Redipuglia, Herbarium Göttingen, GOET001786. 
,In Forojulio ad ripas lapidosas flum. Lisoncii pr. Fogliano Septbr. 1830 leg. Bartling' steht auf dem Originaletikett des Herbarbelegs von Apargia berinii Bartl. (Leontodon berinii (Bartl.) Roth). Die Pflanze wurde demnach im September 1830 in Forojulio in der Nähe des italienischen Ortes Fogliano Redipuglia am steinigen Flussufer des Isonzo gesammelt. Das Etikett selbst suggeriert dabei den Eindruck eines isoliert stattgefundenen Sammlungsprozesses. Denn als Sammler wie auch Beschreibender der Pflanze wird allein der Göttinger Botaniker Bartling genannt. Auf andere Personen wird nicht verwiesen.

Doch anders als die Beschriftung des Pflanzenexemplars als Ergebnis der Exkursion zunächst vermuten lässt, handelte es sich bei der im Jahr 1830 in den Michaelisferien unternommenen wissenschaftlichen Reise nicht um ein von anderen Personen losgelöstes Unterfangen. Die Route von Salzburg bis in das südliche Tirol bewerkstelligte Bartling nicht allein, sondern in Begleitung vier seiner Studierenden. Schon in dem der Reise vorausgehenden Schreiben, in welchem Bartling um eine finanzielle Unterstützung bat, findet dieser Plan Erwähnung:

\footnotetext{
„Dieser unterthänigsten Bitte erlaubt sich der ehrfurchtsvoll Unterzeichnete noch die Bemerkung hinzuzufügen, daß mehrere seiner fleißigsten Zuhörer den Wunsch geäußert haben ihn auf der projectirten Reise zu begleiten und daß er es sich wird angelegen seyn lassen, diesen jungen eifrigen Männern so nützlich als möglich zu werden.“ ${ }^{348}$
}

Auf welche Weise Bartling den Studierenden auf der gemeinsamen Exkursion schließlich ,nützlich geworden ist ${ }^{6}$, gibt auch der nach Abschluss der Reise abgefasste Reisebericht nicht an. ${ }^{349}$ Deutlich wird jedoch, dass das Pflanzenpräparat Apargia berinii seinen Entstehungskontext auf den ersten Blick verschweigt. Sein Bezug zur Lehrpraxis erschließt sich erst durch den Schriftverkehr Bartlings mit dem Kuratorium.

\footnotetext{
348 UniA GÖ, Kur., 5584, Schreiben Bartlings an das Hohe Großbritannisch-Hannoversche Cabinets-Ministerium, Universitäts-Departement, 21.6.1830.

349 Vgl. UniA GÖ, Kur., 5584, Schreiben Bartlings an das Universitätskuratorium, 8.12.1830.
} 


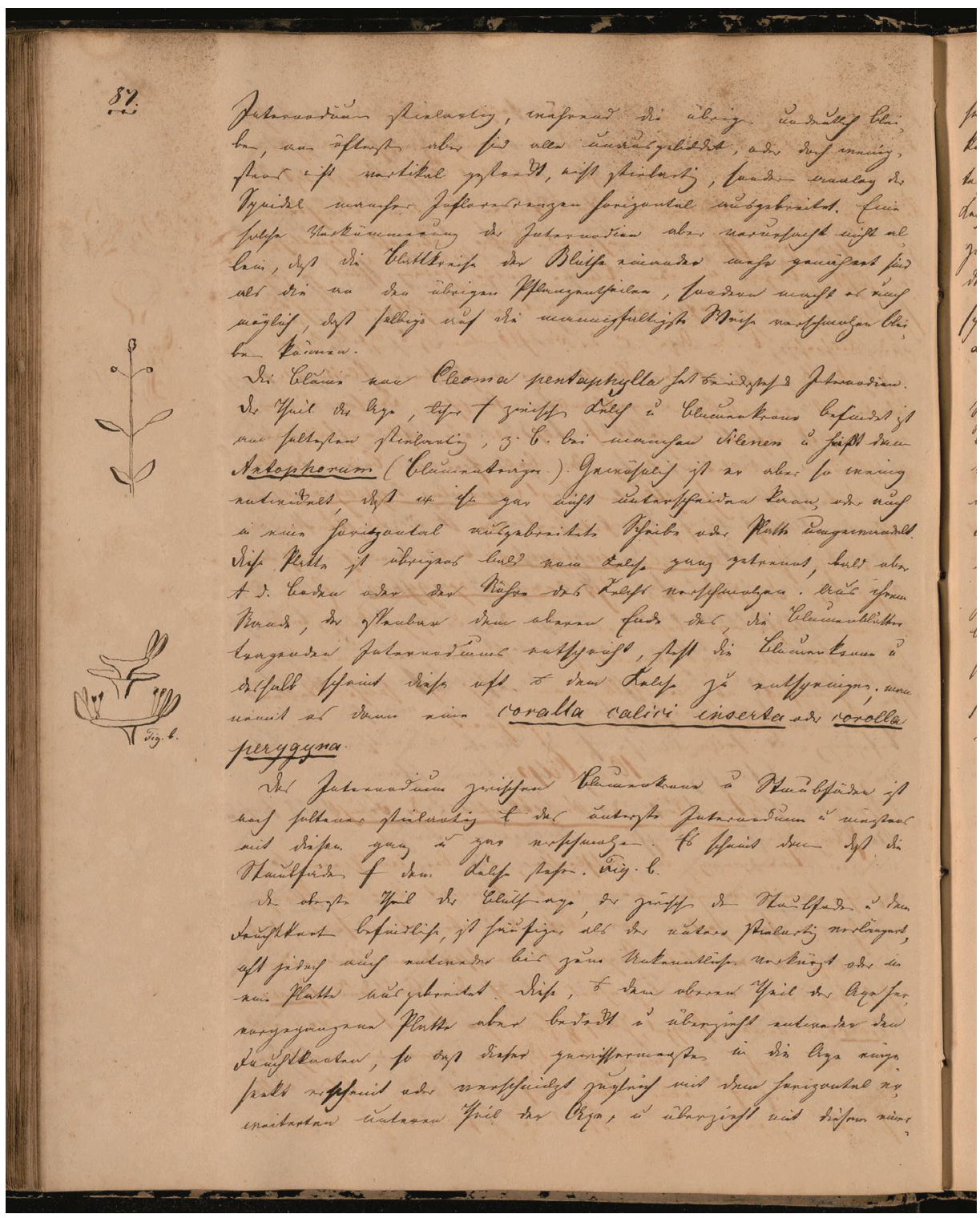

Abb. 15: Seite 87 der Vorlesungsnachschrift des Studierenden Schünemann, Wintersemester 1836/37, Vorlesung über die Organographie und Physiologie der Pflanzen von F. G. Bartling, Staats- und Universitätsbibliothek Göttingen, Handschriftenabteilung, Cod. Ms. 1990.24/1. 


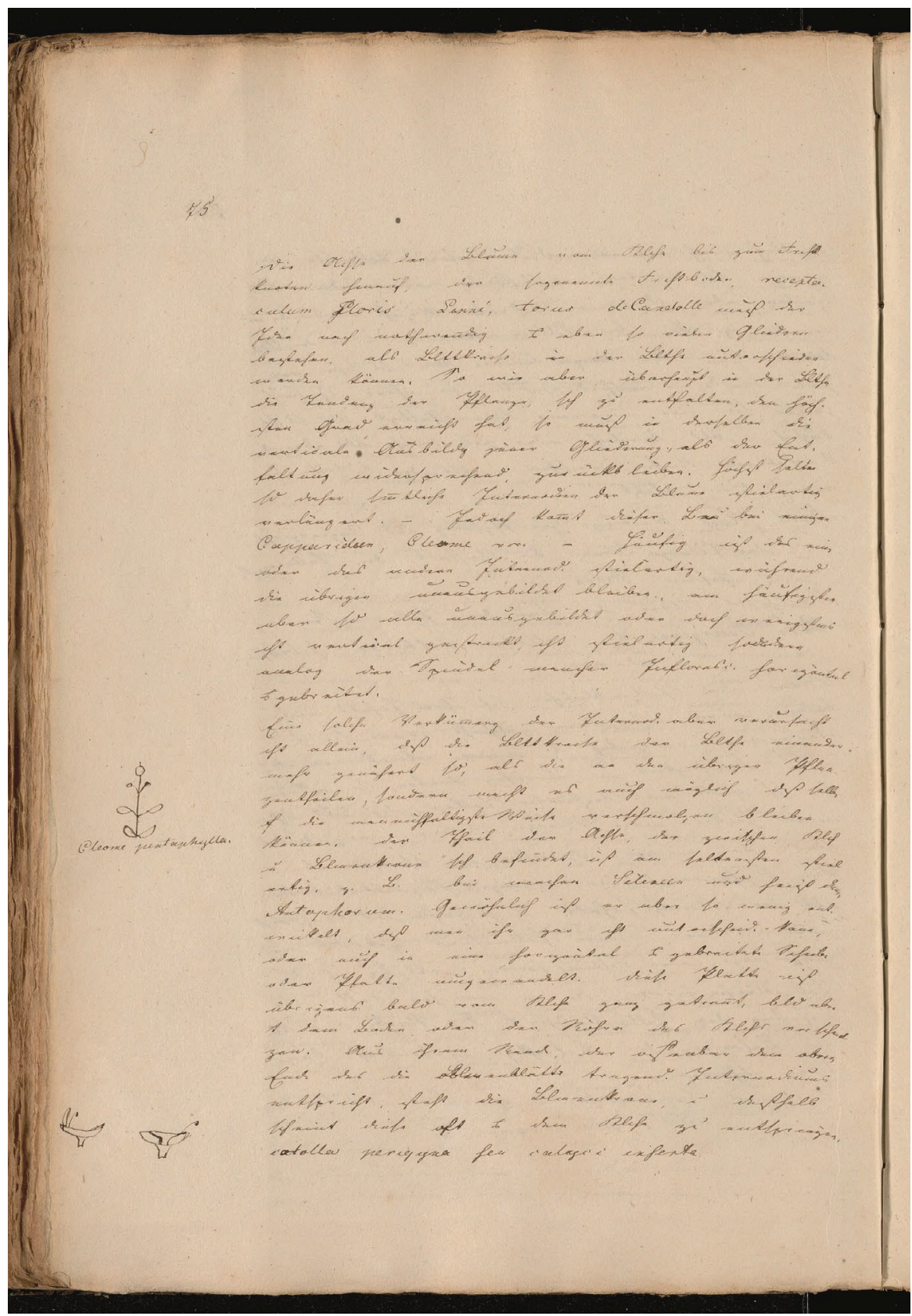

Abb. 16: Seite 75 der Vorlesungsnachschrift des Studierenden W. A. Roth, Wintersemester 1836/37, Vorlesung über die Organographie und Physiologie der Pflanzen von F. G. Bartling, Staats- und Universitätsbibliothek Göttingen, Handschriftenabteilung, Cod. Ms. 2016.12/2. 
Im Wintersemester 1836/37 hielt Bartling eine Vorlesung über die Anatomie und Physiologie der Pflanzen, von der heute zwei Nachschriften zeugen - eine verfasst von dem Studierenden W. A. Roth und die zweite von dem Studierenden Schünemann. ${ }^{350}$ Während Apargia berinii sich nicht sofort als aus dem Lehrkontext stammendes Objekt zu erkennen gibt, verweisen die Schriftquellen hingegen direkt auf die botanische Lehre. Noch während der Veranstaltung oder im Nachhinein abgefasst, geben sie im Fließtext auf deutscher Sprache den Inhalt der Vorlesung wieder. ${ }^{351}$ Beide hier gezeigten Auszüge stammen aus dem neunten Kapitel des Abschnitts zur Organographie, der Lehre von der äußeren Gestalt der Pflanzenbestandteile, das bei Schünemann die Überschrift, Vom Bau der Blüthen` und bei Roth ,Vom Bau der Blumen' trägt. Eine Gegenüberstellung der Auszüge macht offenkundig, dass sich die beiden Nachschriften nicht allein in ihrem Wortlaut entsprechen, sondern auch die an vielen Stellen der Nachschriften flankierend auftretenden Zeichnungen einander gleichen. Schünemann und Roth arbeiteten nach denselben Vorlagen. Ihre Nachschriften belegen, dass die von Bartling abgehaltene Vorlesung nicht allein aus dem gesprochenen Wort bestand, sondern auch Objekte Eingang in die Veranstaltung fanden. An den hier ausgewählten Stellen der beiden Nachschriften wurde sogar Bezug auf eine ganz konkrete Pflanzenart genommen: Die erste von Schünemann gemachte Zeichnung am Seitenrand wurde mit ,Cleome pentaphylla' (Cleome gynandra L.) bezeichnet. Und auch zu dieser Illustration gibt es ein Pendant bei Roth. Die Zeichnung von Schünemann ist 3,5 und die von Roth 1,6 cm lang und genauso wie der Text mit Tinte gefertigt. Anders als Apargia berinii verweisen die Zeichnungen in den Nachschriften damit zwar direkt auf die akademische Lehre als ihren Entstehungskontext, doch lassen sie den Betrachtenden in Unkenntnis über die tatsächlichen Lehrobjekte, die ihnen zugrunde lagen.

350 Vgl. Cod. Ms. 1990.24/1; Cod. Ms. 2016.12/2. Die Vorlesung hielt Bartling montags, dienstags, donnerstags und freitags um jeweils elf Uhr. Vgl. Georg-August-Universität Göttingen 1830-1852, WS 1836, S. 11. Bei den Vorlesungsverzeichnissen handelt es sich allerdings in erster Linie um Ankündigungskataloge. Sie geben keinen Aufschluss darüber, ob die Veranstaltungen auch durchgeführt und welche Themen tatsächlich behandelt wurden. Vgl. Bruning 2011.

351 Beide Vorgehensweisen, sowohl das Notieren während der Veranstaltungen als auch die Ausführung zu einem späteren Zeitpunkt, sind üblich gewesen. Vgl. Joost 2001, S. 38. Neben dem Entstehungskontext bleibt ebenso offen, ob nach einem Lehrbuch gearbeitet wurde, Bartling den Text diktierte oder einen freien Vortrag hielt. Vgl. Paulsen 1965, S. 142-144. 
Neben seiner Funktion als Forschungssammlung galt das Herbarium Bartling von vornherein auch als zentrales Hilfsmittel für den Unterricht im Fach Botanik:

\begin{abstract}
„[F]ür ein gründliches Studium der Botanik [ist] ein möglichst reiches und gut geordnetes Herbarium das erste und wichtigste Hülfsmittel $[\ldots]^{“}$ und ist „,...] nicht allein für die botanischen Studien überhaupt, sondern auch für die Vorlesungen und für die richtige Bestimmung der Pflanzen im botanischen Garten ein solches unentbehrlich [... ]“352.
\end{abstract}

Die Verknüpfung von Sammlung und Lehre fand dabei in dreierlei Weise statt: Zum einen wurden Herbarbelege mit in die Veranstaltungen gebracht und zum anderen wurde durch die von Bartling angestrebte Öffnung der Sammlung auch Studierenden der Zugang zum Herbarium selbst ermöglicht. Gleichzeitig kamen nicht nur die fertigen Objekte zum Einsatz, sondern die Studierenden wurden in den Prozess der Objektgenese selbst mit einbezogen. Damit wurde an bereits bestehende Lehrpraktiken angeknüpft, ebenso wurden aber neue Wege beschritten, die auch nicht von allen Dozierenden gleichermaßen mitgetragen wurden.

Tatsächlich war die Georgia Augusta, wie auch die anderen Hochschulen des späten 18. und frühen 19. Jahrhunderts, keine Forschungsanstalt, wie wir sie heute kennen. Die Lehre bedeutete für die Dozierenden einen solch großen Zeitaufwand, dass für die eigene wissenschaftliche Arbeit wenig Raum blieb. ${ }^{353}$ Das Fehlen umfassender Dokumentationen der Lehrpraxis der in Göttingen wirkenden Botaniker*innen darf deshalb nicht über deren Stellenwert hinwegtäuschen, sondern muss als symptomatisch für diesen Teil universitärer Praxis gesehen werden. ${ }^{354}$ Denn zusätzlich zu ihrer Bedeutung als Teil wissenschaftlicher Tätigkeit galten die Lehrveranstaltungen den Dozierenden, und ganz besonders den Privatdozierenden, als unverzichtbare Einnahmequelle. ${ }^{355}$ Studierende mussten für die Veranstaltungen zahlen und noch bis zum Ende des

\footnotetext{
352 UniA GÖ, Kur., 5592, Schreiben Bartlings an das Universitätskuratorium, 26.2.1845.

353 Vgl. Joost 2001, S. 34.

${ }^{354}$ So gilt die Rekonstruktion der Lehrpraxis nach wie vor als Desiderat der Universitätsgeschichte. Vgl. Bruning 2011, S. 284. Besonders die Vorlesungspraxis ist im Vergleich zur Disputation bisher wenig untersucht worden. Vgl. Füssel 2015b, S. 65. Die Lehre mit Objekten erfuhr zuletzt vermehrt Aufmerksamkeit. Doch wird hier besonders der außeruniversitäre Unterricht in den Blick genommen. Vgl. Bergsträsser \& Markert 2018; Markert 2017; Lehmann 2016. Auch viele der Beiträge aus dem Sammelband Ordnen - Vernetzen - Vermitteln. Kunst- und Naturalienkammern der Frühen Neuzeit als Lehr-und Lernorte aus dem Jahr 2018, der sich auch der universitären Lehre mit Objekten widmet, behandeln die Lehre letztlich nur beiläufig. Vgl. Dolezel et al. 2018.

355 Vgl. Schlumbohm 2016, S. 237; Tütken 2005. Bei den botanischen Vorlesungen handelte es sich offensichtlich vorwiegend um privata, also bezahlte Veranstaltungen. Die öffentlichen Vorlesungen (publica), die noch bis ins 18. Jahrhundert üblich gewesen waren, verloren auch an der Georgia Augusta an Bedeutung. Vgl. Georg-August-Universität Göttingen 1830-1852; Paulsen 1965, S. 130 f.; Saada 2008, S. 45.
} 
19. Jahrhunderts waren die Lehrenden auf diese Einkünfte auch angewiesen. 356 Gleichwohl traten neben finanzielle Beweggründe fachliche Auffassungen und Überzeugungen, die sich besonders in der Ausgestaltung der Lehrveranstaltungen niederschlugen: Neben den Vorlesungen oder auch in deren Rahmen unternahmen die Dozierenden der Botanik Exkursionen mit ihren Studieren und führten Demonstrationen im botanischen Garten sowie am Mikroskop durch. ${ }^{357}$ Damit waren die eigene Anschauung und Beobachtung am Objekt schon vor der Gründung des Herbariums üblich und ein etablierter Bestandteil der Lehre.

Das zeigt sich nicht erst in dem 1837 verfassten Aufsatz Bartlings über den botanischen Garten. ${ }^{358}$ Schon der Eintrag in Friedrich Karl Gottlob Hirschings Sammlungsführer von 1792 thematisiert die Art und Weise der Einbindung von Objekten in die Lehre am Göttinger botanischen Institut. ${ }^{359}$ Dieser beschreibt Demonstrationen und Untersuchungen im Garten als selbstverständlichen Bestandteil der Lehrveranstaltungen, an welche dessen Infrastruktur sogar angepasst wurde. 360 Und auch die Möglichkeit des Gartenbesuchs durch die Studierenden außerhalb der Lehrveranstaltungen war bereits zum Ende des 18. Jahrhunderts in solcher Weise etabliert, dass Hirsching die dafür üblichen Uhrzeiten angeben konnte. ${ }^{361}$ Ebenso wird an dieser Stelle beschrieben, dass man zur Anschauung nicht allein den Garten aufsuchte:

„[...] mehrentheils [werden] die Gewächse im Hörsaale demonstrirt, da man dann durch schlimme Witterung nicht gehindert wird fortzufahren, und da ferner mancherlei erhebliche Erläuterungen durch Zeichnungen, Bücher, trokene Pflanzen, u. s. w. sich können anbringen lassen, auch die Gefahr der zufälligen Beschädigung verringert wird, und die Lernbegierigen einander nicht den Prospect nach dem zu untersuchenden Gegenstande verhindern können." ${ }^{362}$

Deutlich wird, dass die doppelte Einbindung von Objekten - der Weg der Objekte in die Veranstaltung sowie die Verlagerung der Lehre in die Sammlung selbst - schon vor der Gründung des Herbariums bestand. Doch auch wenn bereits an dieser Stelle die getrockneten Pflanzen Erwähnung fanden, wird die

\footnotetext{
356 Vgl. Joost 2001, S. 32.

357 Vgl. Georg-August-Universität Göttingen 1830-1852. Mikroskopische Demonstrationen sind ab dem Wintersemester 1841 nachzuweisen. Sie wurden zunächst nur von Grisebach, in der Folge aber auch von Bartling und Lantzius-Beninga angeboten. Vgl. Georg-August-Universität Göttingen 1830-1852, WS 1841, S. 11.

358 Vgl. Bartling 1837.

359 Vgl. Hirsching 1792, S. 124-170.

360 Vgl. ebd., S. 155, S. 157 f. u. 164.

361 „Die gewöhnliche Zeit, da der Garten von den Studierenden besucht wird, sind im Sommer die Abendstunden von 5. bis 7. Uhr, den Sonnabend ausgenommen." Ebd., S. 165. Zur Nutzung des botanischen Gartens vgl. außerdem PWI.Syst.Geobotan.A19, Gesetze für die Studierenden auf der Georg-August-Universität zu Göttingen (1830), Regulativ über die Benutzung des botanischen Gartens zu Göttingen, S. 66-67.

362 Ebd., S. 155.
} 
offenbare Vorrangstellung des botanischen Gartens und damit der lebenden Pflanzenexemplare in der Lehre deutlich. Der Einsatz von Herbarbelegen ist folglich vor 1832 üblich gewesen, doch handelte es sich dabei im Vergleich zum Garten um eine einseitige Laufrichtung: Für die eigene Anschauung wurden die Studierenden in erster Linie auf Naturbeobachtungen und damit auf den botanischen Garten verwiesen. Eine Einsicht in die Herbarien der Göttinger Dozierenden außerhalb der Vorlesungen blieb in erster Linie anderen Wissenschaftler*innen vorbehalten. Dies verschob sich erst mit der Gründung des Herbariums allmählich. Und während Schrader dieser Entwicklung argwöhnisch entgegentrat, setzte sich Bartling intensiv für den Ausbau der Sammlung und ihre Nutzung sowohl in der Forschung als auch in der Lehre ein.

Die Einbindung der Herbarbelege in die Lehre setzte nicht erst nach ihrer Erstellung ein, sondern begann schon einige Arbeitsschritte zuvor. Die Beobachtung in der Natur und an der lebenden Pflanze wurde nicht ausschließlich im botanischen Garten gelehrt. Die Dozierenden unternahmen im Rahmen der Veranstaltungen ebenso Exkursionen mit ihren Studierenden. ${ }^{363}$ Und auf diesen Ausflügen wurde nicht nur Anschauung betrieben, sondern auch Pflanzenmaterial gesammelt, das in der Folge seinen Weg in die Sammlung fand. Das Pflanzenexemplar von Apargia berinii, das zweifelsfrei einer mit Zuhörern unternommenen Reise Bartlings zuzuordnen ist, steht stellvertretend für jene Beteiligung der Studierenden am Prozess der Akquirierung. Die groß angelegte Exkursion, die, von Salzburg ausgehend, durch das Tal der Salzach, nach Wildbad in Gastein, über die Zentralkette der Alpen, in die Umgebung des Groß-Glockners und von dort durch das Tal der Piave, über Belluno, nach Triest und schließlich nach Venedig führte, stellte allerdings in zweifacher Weise eine Ausnahme dar. ${ }^{364}$ Zum einen handelte es sich um die erste Forschungsreise Bartlings in eine entferntere Region nach mehr als zehn Jahren. Zum anderen ist es mit Blick auf nachfolgende Reiseberichte mit großer Wahrscheinlichkeit eine Ausnahme gewesen, dass Bartling auf einer solchen wissenschaftlichen Reise von Studierenden begleitet worden ist. Denn die gemeinsam mit Studierenden stattfindenden Exkursionen hatten in der Regel die Region um Göttingen zum Ziel und fielen damit weitaus kleiner aus. Wie sie in der Praxis konkret ausgestaltet worden sind, lässt sich nicht rekonstruieren, da für die vornehmlich kleineren Ausflüge keine Dokumentationen vorliegen. Damit muss offenbleiben, ob die Studierenden hier selbst sammelten und wenn ja, ob das Gesammelte in privater Hand blieb oder am Ende vielleicht auch in das Herbarium gelangte. Im Zuge der Exkursionen wurden aber mit Sicherheit, wie schon der von Bartling formulierte Wunsch, den Männern

\footnotetext{
363 Dies ist sowohl für Schrader als auch für Bartling, Lantzius-Beninga und Grisebach zu belegen.

Vgl. Georg-August-Universität Göttingen 1830-1852; o. A. 1838, S. 356; UniA GÖ, Kur., 5592, Schreiben Bartlings an das Universitätskuratorium, 15.2.1847.

${ }^{364}$ Vgl. UniA GÖ, Kur., 5584, Schreiben Bartlings an das Universitätskuratorium, 8.12.1830.
} 
„so nützlich als möglich“365 werden zu wollen, andeutete, auch Kenntnisse darüber gewonnen, welche Voraussetzungen eine Pflanze mitbringen musste, die sich als Präparat eignen sollte, wie Pflanzen demnach auszuwählen und wie sie noch während der Reise zuzurichten seien. Das mag durch Beobachten, aber sicherlich auch durch eigenes Sammeln vermittelt worden sein - auf der Exkursion nach Südtirol sowie beim Besuch der um Göttingen gelegenen Ausflugsziele.

Ein noch intensiveres Lernen mit Herbarbelegen wurde dann einem ganz ausgewählten Kreis von Studierenden ermöglicht. Tatsächlich handelte es sich dabei um nur jeweils einen Studenten, den Assistenten am Herbarium. Diese Tätigkeit ist dabei nicht als Lehre im strengeren Sinne zu verstehen, doch wurde die Assistentenstelle vermutlich nur von denjenigen Studierenden bekleidet, die sich als besonders engagiert hervortaten. Die drei ersten durch das Kuratorium offiziell genehmigten und heute bekannten Assistenten am Universitätsherbarium, die im Zeitraum von 1843 bis 1873 nacheinander für die Sammlung arbeiteten, Lantzius-Beninga, Oscar Drude und Johannes Reinke (1849-1931) - bekleideten im Anschluss an ihre Tätigkeit in der Sammlung auch ausnahmslos die Position eines Dozierenden. ${ }^{366}$ Dabei ist ihre Assistenz am Herbarium nicht allein vor dem Hintergrund einer speziellen zusätzlichen Bildung einiger weniger zu sehen, sondern weiter gefasst auch als erste Öffnung der Sammlung für Studierende überhaupt zu betrachten. Denn auch wenn neben dem Ausbau der Sammlung besonders ihre Öffnung ein zentrales Ziel darstellte, erreichte ihre Zugänglichkeit lange Zeit nicht das von Bartling angestrebte Ausmaß. Das Herbarium und seine Objekte mussten den neu formulierten Wertmaßstäben und Richtlinien entsprechend zunächst hergerichtet werden und erforderten dann während der Besuchszeiten eine Beaufsichtigung. Abgesehen vom Assistenten des Herbariums fanden deshalb in den ersten Jahren gar keine und in der Folgezeit erst nach und nach Studierende den Weg in die Sammlung.

Es ist deshalb davon auszugehen, dass die Einbindung der Herbarbelege in die Lehre zunächst auch weiterhin vor allem durch das Mitbringen von Objekten in die Vorlesungen stattfand. ${ }^{367}$ Das nur zögerliche Fortschreiten des

365 UniA GÖ, Kur., 5584, Schreiben Bartlings an das Hohe Großbritannisch-Hannoversche Cabinets-Ministerium, Universitäts-Departement, 21.6.1830.

366 Vgl. Wagenitz 1988, S. 48, 106 u. 144.

367 Dabei ist bereits der Professorenhaushalt als Veranstaltungsort der Vorlesungen und anderer Zusammenkünfte zwischen Dozierenden und Studierenden als semi-öffentlicher Wissensraum zu verstehen. Vgl. Mariss 2018, S. 217 f.; Napolitani 2018, S. 113-117. Doch von der selbstständigen Nutzung der Sammlung blieben die Studierenden dennoch ausgeschlossen. Durch die Entstehung von Auditoriengebäuden und Hörsälen, deren Nutzung im Falle Grisebachs auch konkret nachzuweisen ist, trat die Bedeutung des Privathaushaltes für die universitäre Lehre des 19. Jahrhunderts dann insgesamt zurück. Vgl. 2 Cod. Ms. philos. 182: A. H. R. Grisebach, Vorlesungsankündigung von ca. 1850; UniA GÖ, Sek., 528, Tabelle der öffentlichen Hörsäle für das Sommersemester 1849; UniA GÖ, Sek., Tabelle über die öffentlichen Auditorien für das Wintersemester 1849/50. 
Öffnungsprozesses der Sammlung lag dann aber nicht nur in der Anspruchshaltung Bartlings begründet. Vielmehr spiegelte sich darin auch das gespannte Verhältnis zwischen Universitätssammlung und Privatsammlung wider. Denn während Bartling auf der einen Seite eine Auflösung der Privatsammlungen zugunsten eines Universitätsherbariums wünschte, legten die Richtlinien der Sammlung auf der anderen Seite fest, dass nur ihr Vorsteher diese auch uneingeschränkt nutzen durfte. Ausleihen konnten zwar vorgenommen werden, mussten aber stets durch Bartling genehmigt werden. ${ }^{368}$ Und da diese Regelung auch für Dozierende galt, war mit Gründung des Herbariums die Privatsammlung noch lange nicht abgelöst worden. ${ }^{369} \mathrm{Ihr}$ Fortwirken und ihre Bedeutsamkeit reichten auch in Göttingen bis weit in das 19. Jahrhundert. 370

Dies tritt sehr deutlich im Rahmen der Verhandlungen bezüglich Grisebachs Mitwirken im Herbarium und dessen zugrunde liegenden Bedingungen hervor:
„Da meine Thätigkeit hiernach jenem Institute zu Gute kommt, ohne mir als Lehrer irgend welche Vortheile zu verschaffen, da ich namentlich nach Bestimmung 2. nicht als Mitdirector [...], sondern nur als Assistent des Prof. Bartling zu betrachten bin, so findet meines Erachtens der Grundsatz, nach welchem die Directoren von Sammlungen keine eigenen Sammlungen besitzen dürfen, auf mich keine Anwendung. Es werden hierdurch übrigens auch die Interessen des Directors auf keine Weise verletzt, indem ich zu Vorlesungen überall keine getrocknete Pflanzen des Universitäts-Herbarii benutzen werde und zu wissenschaftlichen Zwecken auch meine Privat-Sammlung Herrn Prof. Bartling stets, wie bisher, zu Gebote steht.“ 371

Der Auszug aus dem Schreiben vom 15. Juli 1846 zeigt, dass Grisebach seine private Sammlung, anders als von Bartling gewünscht, nicht im Zuge seiner Anstellung am Herbarium abzugeben gedachte. Die allmähliche Ablösung der Privatsammlung schien ihm eine unliebsame Entwicklung gewesen zu sein und die Modalitäten der Nutzung des Herbariums waren offensichtlich noch nicht in dem Maße ausgereift, als dass man tatsächlich gewillt gewesen wäre, die eigene Sammlung sowohl für Forschungszwecke als auch für die Lehre aufzugeben. Dass es sich um eine schrittweise Entwicklung handelte, zeigt sich nicht nur am Beispiel Grisebachs und Schraders, sondern auch bei Bartling als Vorsteher der neu

368 Vgl. UniA GÖ, Kur., 5584, Schreiben Bartlings an das Universitätskuratorium, 14.11.1832, Anlage A, B, Von der Benutzung des Unviersitäts-Herbarii.

369 Auf ähnliche Prozesse macht auch Samuel Alberti aufmerksam, wenn er beschreibt, dass die Überführung von Privatsammlungen in institutionelle Strukturen häufig nicht reibungslos verlief, keine vollständige Übergabe der Sammlung vollzogen wurde oder geteilte Eigentumsverhältnisse vorlagen. In seinem Artikel Owning and collecting natural objects in nineteenth-century Britain plädiert er deshalb für die Auflösung einer einfachen Dichotomie von privaten und öffentlichen Sammlungsbeständen. Da oft keine Trennschärfe möglich ist, sollten seiner Meinung nach vielmehr die Abstufungen zwischen diesen beiden Polen betont werden. Vgl. Alberti 2005.

370 Vgl. Mariss 2018, S. 217 f.

371 UniA GÖ, Kur., 5584, Schreiben Grisebachs an den Geheimen Kabinettsrat, 15.7.1846. 
angelegten Sammlung. ${ }^{372}$ Denn wenngleich dieser wiederholt betonte, „,...] sowohl für den botanischen Garten, als für [s]eine Vorlesungen und litterarischen

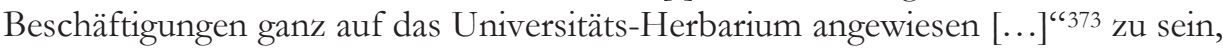
nutzte auch er bis zu dessen Ankauf durch die Universität im Jahr 1841 sein privates Herbarium für die Lehre. ${ }^{374}$

Doch welche Herbarbelege fanden genau Verwendung? Wie und an welcher Stelle kamen sie zum Einsatz? Und was versprachen sich die Dozierenden von ihrer Einbindung in die Lehrveranstaltungen? Während die Nutzung von getrockneten Pflanzenpräparaten an sich durch viele Schreiben zu belegen ist, ist der konkrete Einsatz der Objekte nur eingeschränkt nachzuweisen. Zwar zeigen schon die Aushandlungen über den Besitz von Privatsammlungen, dass die für die Veranstaltungen genutzten Belege nicht vordergründig aus dem Universitätsherbarium, sondern aus den Sammlungen der Dozierenden bezogen wurden. Wie die Pflanzenbelege aber genau in die Lehre eingebunden worden sind, wurde an keiner Stelle dokumentiert.

Einige wenige Auszüge aus Schreiben, die an das Kuratorium in Hannover gerichtet worden sind, geben zumindest Hinweise auf die Objekte, die Bartling für seine Lehrveranstaltungen gebrauchte, und welche Anforderungen er an diese stellte. Im Zuge der vom Kuratorium angeregten Wertprüfung vor dem Ankauf seiner Privatsammlung äußerte Bartling beispielsweise, diese vor einer Weitergabe zunächst umarbeiten zu müssen:

„Dieser Bericht [gemeint ist die Einschätzung der Sammlung durch den Hofrat Meyer] hat deshalb noch nicht abgefasst werden können, weil einige Theile meines Herbariums, die ich bis jetzt noch immer zu den Vorlesungen benutzt habe, bevor sie übersehen und taxirt werden können, einer Umarbeitung bedürfen, zu welcher ich erst in diesem Winter Zeit zu gewinnen hoffen darf.“"375

An dieser Stelle bringt Bartling also zum Ausdruck, dass die in der Lehre Verwendung findenden Herbarbelege sich in einer anderen Form befanden als der Rest seines Herbariums. Wie sich die Belege voneinander unterschieden und worin die erforderliche Umarbeitung genau bestand, ob sie in der Zurichtung oder Bezeichnung der Präparate lag, oder doch andere Punkte betraf, bleibt unklar. Aus einem Schreiben Bartlings geht zumindest hervor, welche Pflanzen bevorzugt von ihm genutzt wurden. So bezeichnet er die Moose, Flechten und anderen Kryptogamen als „bei den Vorlesungen unentbehrliche [...] Sammlungen“376.

\footnotetext{
372 Auch Schrader nutzte für Vorlesungen seine Privatsammlung. Vgl. o. A. 1838, S. 356.

373 UniA GÖ, Kur., 5584, Schreiben Bartlings an den Geheimen Kabinettsrat, 30.6.1846.

$374 \mathrm{Vgl}$. UniA GÖ, Kur., 5592, Schreiben Bartlings an das Universitätskuratorium, 14.12.1840.

375 UniA GÖ, Kur., 5592, Schreiben Bartlings an das Universitätskuratorium, 14.12.1840.

376 UniA GÖ, Kur., 5592, Schreiben Bartlings an das Universitätskuratorium, 15.4.1844.
} 
Die einzigen Quellen aber, die von den Veranstaltungen selbst zeugen, sind die zu den Vorlesungen von Schrader, Bartling und Grisebach vorliegenden Vorlesungsnachschriften. ${ }^{377}$ In Form von Fließtexten geben sie zuvorderst Aufschluss über den vermittelten Inhalt. Wie jedoch die Nachschriften zu Bartlings Vorlesung aus dem Wintersemester 1836/37 beispielhaft zeigen, wurden die Texte an vielen Stellen durch Zeichnungen der Studierenden ergänzt. Diese werden wie bei der Zeichnung von Cleome pentaphylla zwar in manchen Fällen mit einem wissenschaftlichen Namen versehen. Auf welches konkrete Objekt sie sich beziehen, ob nach Vorlage eines Buches, einer Lehrtafel oder doch eines Herbarbelegs gearbeitet worden ist, bleibt dennoch unklar. Anhand der Vorlesungsverzeichnisse wird allerdings deutlich, dass neben dem Zusammenhang zwischen Jahresverlauf und Objektnutzung auch ein Einfluss auf die Vorlesungsinhalte bestand. Denn einige Themen wurden vornehmlich im Wintersemester und andere im Sommersemester abgehalten. ${ }^{378}$ Und auch Bartling äußerte, dass die Veranstaltungen im Wintersemester ein geringeres Einkommen einbrächten. ${ }^{379}$ Dass die zur Verfügung stehenden Objekte die Ausgestaltung der Lehre maßgeblich mitbestimmten, liegt deshalb nahe. Um darüber hinaus aber auch eine Entwicklung in der Ausführung der Zeichnungen feststellen zu können, liegen nicht ausreichend Nachschriften vor. Auffallend ist zumindest, dass die Zeichnungen der Vorlesungen Bartlings ausschließlich zweidimensional sind, während die zu Schraders Vorlesung im Sommersemester 1821 und Wintersemester 1822 angefertigten Zeichnungen (Abb. 17) dreidimensionale Objekte zeigen und die Nachschriften sogar getrocknete Pflanzen enthalten (Abb. 18). ${ }^{380}$

377 Vgl. Cod. Ms. 1990.24/1; Cod. Ms. 1990.24/2; Cod. Ms. 2016.12/2; 4 Cod. Ms. hist. nat. 59 a, Angeb.; 4 Cod. Ms. hist. nat. 63 d : 13; 4 Cod. Ms. hist. nat. 101 d; 4 Cod. Ms. hist. nat. Nachtr. 10; 4 Cod. Ms. hist. nat. Nachtr. 11; 8 Cod. Ms. hist. nat. 63 e; 8 Cod. Ms. philos. 13 o. Vorlesungsnachschriften garantieren allerdings keine fehlerfreie Rezeption und müssen dahingehend kritisch gelesen werden. Vgl. Joost 2001, S. 34.

378 Allgemeine, spezielle, medizinische, landwirtschaftliche sowie ökonomische und Forst-Botanik wurden ausschließlich im Sommersemester gelehrt, während die Vorlesungen über die Anatomie und Physiologie der Pflanzen und die Kryptogamen im Wintersemester abgehalten wurden. Exkursionen fanden das ganze Jahr über statt. Die Demonstrationen im Garten wurden im Wintersemester durch Besuche in die Gewächshäuser sowie mikroskopische Demonstrationen ersetzt. Vgl. Georg-August-Universität Göttingen 1830-1852.

379, „[... [ da einerseits theils nur im Sommer die botanischen Vorlesungen einen Erwerb darbieten, theils zum Studium der Botanik kostspielige Sammlungen und eine ausgedehnte eben so kostspielige Correspondenz unumgänglich nothwendig sind, anderseits aber schriftstellerische Arbeiten wohl in keinem Fache so wenig lohnen sind; als in diesem.", UniA GÖ, Kur. Pers., 5828, Schreiben Bartlings an den Geheimen Kabinettsrat, 11.3.1832; „Durch diese gnädige Bewilligung in den Stand gesetzt, in dem beginnenden Wintersemester, in welchem mein Fach nur ein geringes Einkommen gewähren kann, meinen Vorlesungen und Privat-Arbeiten ohne drückende Sorgen mich widmen zu können, wage ich es, [...] das Gefühl des innigsten Dankes für die mir zu Theil gewordene hohe Gnade ehrerbietigst auszusprechen [...].“, UniA GÖ, Kur. Pers., 5828, Schreiben Bartlings an das Universitätskuratorium, 7.11.1835.

${ }^{380}$ Die auf Abb. 17 gezeigte Pflanze wurde, wie die Abdrücke zeigen, sogar in der Nachschrift selbst gepresst. 


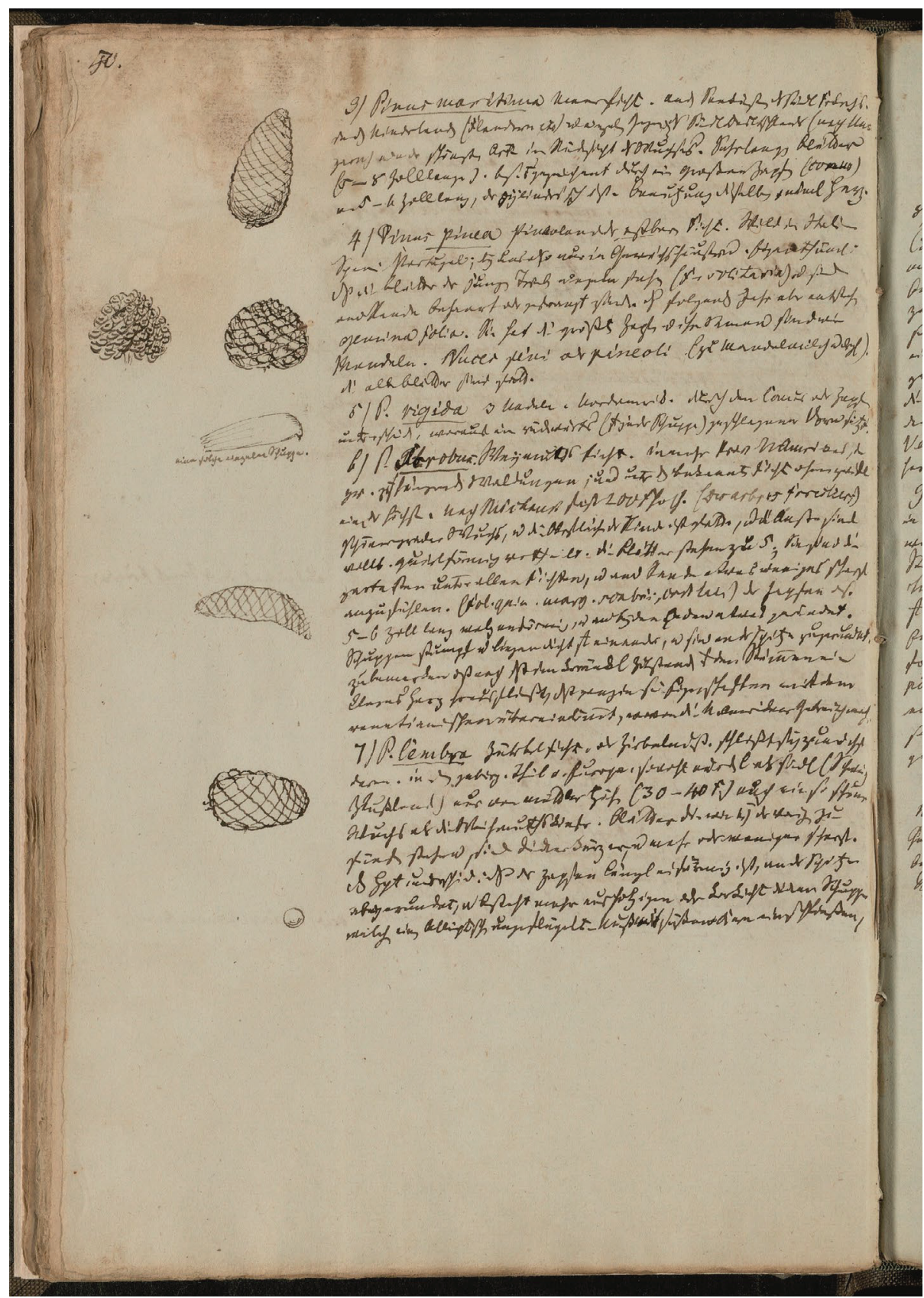

Abb. 17: Seite 50 der Vorlesungsnachschrift des Studierenden G. Schwalbe, Sommersemester 1821, Staats- und Universitätsbibliothek Göttingen, Handschriftenabteilung, 4 Cod. Ms. hist. nat. 63 d: 13. 


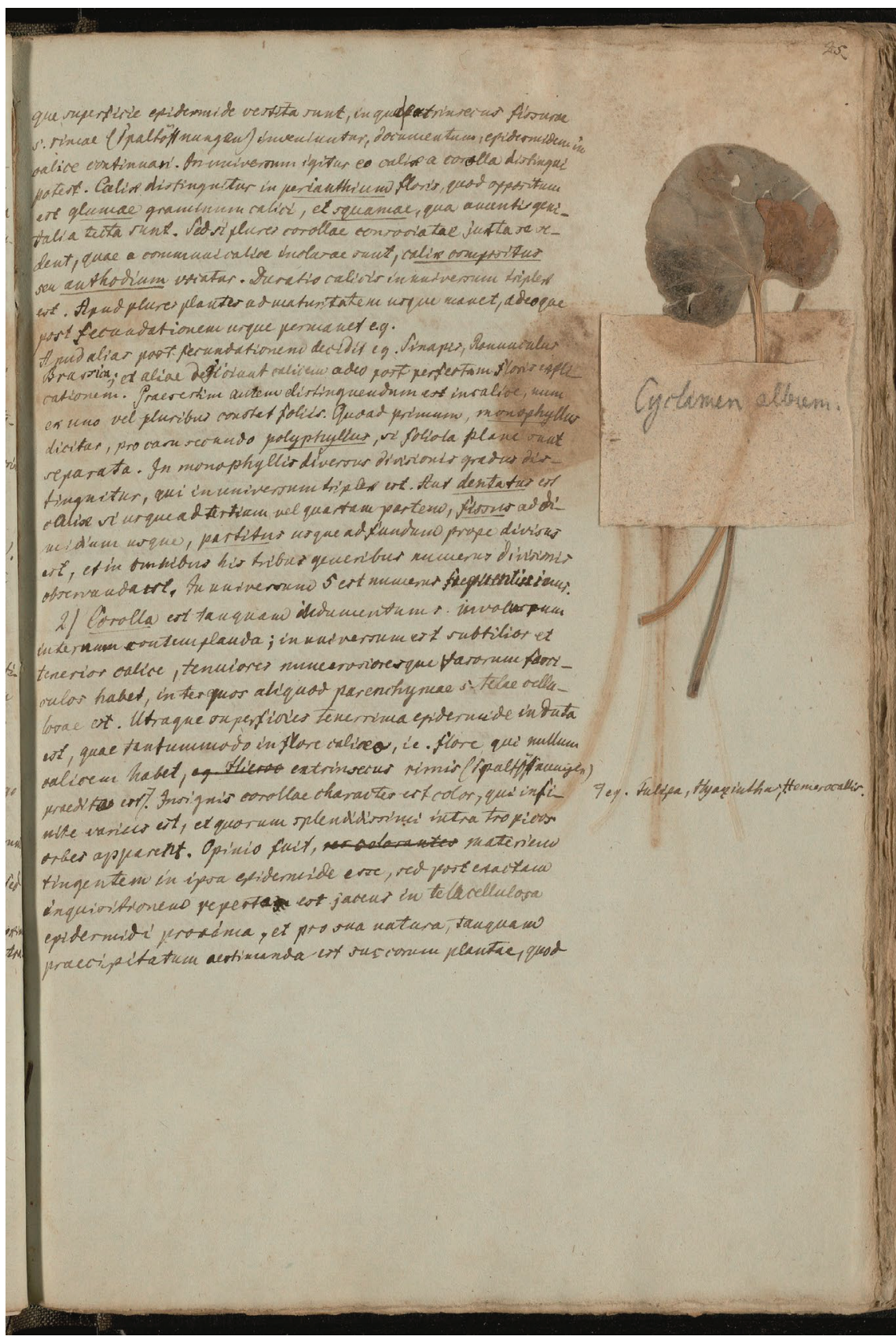

Abb. 18: Seite 25 der Vorlesungsnachschrift des Studierenden G. Schwalbe, Sommersemester 1821, Staats- und Universitätsbibliothek Göttingen, Handschriftenabteilung, 4 Cod. Ms. hist. nat. 63 d: 13. 
Doch auch wenn der Einsatz getrockneter Pflanzenexemplare in der Lehre nicht konkreter zu belegen und damit nachzuvollziehen ist, geben die zur Verfügung stehenden Informationen sehr wohl Aufschluss über Entwicklungen und Tendenzen, die sich in der botanischen Lehre mit Objekten im betrachteten Zeitraum von 1832 bis 1852 abzeichneten. Das verdeutlichen auch die eingangs beschriebenen Objekte: Denn selbst wenn Apargia berinii ihren Bezug zur Lehrpraxis ohne ein Hinzuziehen zusätzlicher Dokumente nicht offenbar macht, liegt schon ihrem Entstehungskontext eine grundlegende Verschiebung in der Lehre des botanischen Instituts zugrunde. Es wurde bereits deutlich gemacht, dass die unter der Leitung Bartlings durchgeführte Exkursion, in dessen Rahmen die Pflanze gesammelt worden ist, nicht den üblichen Ausflügen im Rahmen der Lehrveranstaltungen entsprach. Und dennoch drückt sich hier ein zentraler Punkt im Wandel der Lehrpraxis exemplarisch ab. Nach einer langen Phase ohne groß angelegte Exkursionen bat Bartling um Genehmigung einer Reise, die darüber hinaus zum Ziel hatte, Pflanzenmaterial für die bereits damals von ihm geplante Einrichtung eines öffentlichen Herbariums zusammenzutragen. ${ }^{381}$ Für seine Forschungstätigkeit sowie das von ihm mit viel Einsatz verfolgte Ziel, eine solche Sammlung zu etablieren, muss die Forschungsreise von 1830 einen hohen Stellenwert besessen haben. Und trotz dessen ließ er sich gerne von Studierenden begleiten und öffnete damit gleichermaßen seine Forschungstätigkeit nach außen. Diese Öffnung schlug sich, wenn auch auf andere Weise, ebenso in den Vorlesungsnachschriften nieder. Das tritt nicht so sehr in einer alleinigen Betrachtung der zu Bartlings Veranstaltungen abgefassten Exemplare hervor, wird aber sehr deutlich, wenn man die beiden zu Schraders Vorlesungen erhaltenen Schriften vergleichend hinzuzieht: Denn während die Nachschrift aus dem Sommersemester 1824 ausschließlich in deutscher Sprache verfasst wurde, entwickelte sich der Text aus dem Sommersemester 1821 und dem Wintersemester 1822/23 erst von vorwiegend lateinischer Sprache über vereinzelte Begriffsübersetzungen hin zu einer rein deutschsprachigen Nachschrift. ${ }^{382}$ Dass es sich dabei nicht um eine Ausnahme, sondern eine Verschiebung größeren Ausmaßes handelte, zeigt sich dann in den Promotionsschriften und selbst in den

381 Vgl. UniA GÖ, Kur., 5584, Schreiben Bartlings an das Hohe Großbritannisch-Hannoversche Cabinets-Ministerium, Universitäts-Department, 21.6.1830. Dass dieser Plan auch zur Umsetzung gebracht wurde, zeigt Bartlings Kommentar zu den Ergebnissen der Reise: „Diese Sammlung, deren wichtigste Bestandtheile aus Oberkärnthen, aus den Karnischen Alpen und vom adriatischen Meere sind, besteht etwa aus 500 Arten und 2-3000 Exemplaren und dürfte sich wohl als Beitrag zu einem öffentlichen Herbarium eignen, wenn Eure Excellenzen ein solches in Göttingen einrichten zu lassen geruhen sollten, und würde der ehrfurchtsvoll Unterzeichnete sich glücklich schätzen, wenn er auf diese Weise sein Scherflein zu einem Institute beitragen könnte, das für die in Göttingen Botanik Treibenden ein erwünschtes Hülfsmittel seyn müßte.“ UniA GÖ, Kur., 5584, Schreiben Bartlings an das Universitätskuratorium, 8.12.1830.

382 Vgl. 4 Cod. Ms. hist. nat. 63 d: 13; 8 Cod. Ms. hist. nat. 63 e. 
Publikationen der Dozierenden selbst. ${ }^{383}$ Der Unterricht wurde nicht mehr auf Latein vollzogen und damit einhergehend zugänglicher gestaltet. ${ }^{384}$

Bartlings diesbezügliches Engagement weist die naheliegende Schlussfolgerung, sein Antrieb sei allein aus finanzieller Abhängigkeit und dem damit verbundenen Wunsch nach einer Zunahme der Teilnahmezahlen geboren, entschieden zurück. Vielmehr schienen fachliche sowie ideelle Ansichten sein Handeln maßgeblich mitbestimmt zu haben. Bartling sprach den Studierenden einen neuen Stellenwert und damit auch neue Rechte zu. Das zeichnete sich in der Ausgestaltung der Lehre sowie im Umgang mit den dort Verwendung findenden Objekten ab. Schon Schrader sprach sich für ein Beobachten in der Natur aus und bot selbst regelmäßig Demonstrationen im botanischen Garten an. ${ }^{385}$ Doch diese Aspekte der botanischen Lehre wurden von Bartling weiter ausgebaut, womit er in Widerspruch zu seinem dienstälteren Kollegen trat. Bartling verfolgte offensichtlich eine inhaltliche Öffnung und die Ermöglichung einer Lehre, die sich an eben jenen Forschungsstandards orientierte, die auch die wissenschaftliche Arbeit der Dozierenden selbst leiteten.

In der Institutionalisierung des Herbariums als öffentliche Sammlung scheinen diese Punkte zusammenzulaufen: Der zunehmenden Bedeutsamkeit getrockneter Pflanzenbelege wurde mit der Gründung dieser Universitätssammlung Rechnung getragen. Wissenschaftler*innen und Studierende benötigten gleichermaßen einen Zugang zum Herbarium, um mit den Objekten zu lernen und zu arbeiten. ${ }^{386}$ So wurden die Herbarbelege also bereits vor der Gründung der Sammlung in der Lehre genutzt, sollten nun aber den Studierenden selbst verstärkt zugänglich gemacht werden. Durch explizit der Sammlung verpflichtete Assistentenstellen wurden einzelne Studierende darüber hinaus in die Sammlungsarbeit eingebunden und damit nicht nur an die Erstellung der Sammlungsobjekte, sondern in manchen Fällen auch an deren Beforschung herangeführt. Damit traten das Herbarium und die Pflanzenpräparate auch in der Lehre neben den botanischen Garten.

\footnotetext{
383 Vgl. Wagenitz 1988, S. 10. Auch in der gemeinsamen Publikation von Bartling und Wendland von 1824 wird zwischen Latein und Deutsch gewechselt. Dazu schreiben die Autoren im Vorwort des Bandes: „Uebrigens werden wir uns, je nachdem es passend scheint, bald der lateinischen, bald der deutschen Sprache bedienen“. Bartling \& Wendland 1824, S. VII.

384 Vgl. Paulsen 1965, S. 135 u. 148. Womöglich kann auch die Unterteilung der Vorlesungen Bartlings in unterschiedliche Kapitel im Sinne einer solchen Ausrichtung der Veranstaltungen auf die Bedürfnisse und Anforderungen der Studierenden hin verstanden werden.

385 Vgl. UniA GÖ, Kur., 5584, Schreiben Schraders an den Obermedizinalrat Blumenbach, 31.3.1832; o. A. 1838 , S. 356.

386 Ein Beispiel für eine solche institutionelle Öffnung stellte auch die Göttinger Universitätsbibliothek dar, die den Studierenden täglich offen stand und es ihnen genauso wie den Dozierenden erlaubte, Literatur auszuleihen. Vgl. Paulsen 1965, S. 13 u. 141. Und auch an der Universitätsbibliothek waren Studierende (zum Beispiel Lantzius-Beninga) als Assistenten tätig. Vgl. Wagenitz 1988, S. 106.
} 


\section{Schluss}

„Es ist [...] allgemein anerkannt, daß die botanischen Gärten in der letztern Beziehung [gemeint ist die Förderung der systematischen Botanik] wenig geleistet haben und daß selbst die möglichen Leistungen nicht in Verhältniß zu den Kosten stehen, welche ihr Unterhalt und die Vermehrung des Pflanzenvorraths erfordern. Kein einziges systematisches Werk von Bedeutung ist in der neuren Zeit aus der ausschließlichen Benutzung botanischer Gärten hervorgegangen, diese Richtung der Wissenschaft gehört einer vorübergegangenen Periode an.“ ${ }^{\text {“387 }}$

Zu diesem Fazit kam Grisebach 1875 - also 23 Jahre nach der Rückkehr des Herbariums in den botanischen Garten. In seinem Schreiben an den königlichen Senatsminister und Minister der geistlichen, Unterrichts- und MedizinalAngelegenheiten in Berlin stellte er damit deutlich heraus, dass er den Gärten als Lehrmittel sowie für wissenschaftliche Untersuchungen nur marginale Bedeutung zuschrieb. Für die systematische Botanik seien vielmehr Herbarien sowie pflanzenphysiologische Untersuchungen von Relevanz. ${ }^{388}$ Und dabei handele es

\footnotetext{
387 UniA GÖ, Kur. Pers., 4973, Schreiben Grisebachs an den königlichen Senatsminister und Minister der geistlichen, Unterrichts- und Medizinal-Angelegenheiten Herrn Dr. Falk in Berlin, 11.12.1875.

388 Die vordergründigen Aufgaben des Direktors des botanischen Gartens und des damit verbundenen Herbariums bestanden für Grisebach nämlich in der Durchführung von
} 
sich nicht um seine persönliche Einschätzung, sondern um eine ,allgemein anerkannte' Auffassung.

Verglichen mit den um 1832 verfassten Gesuchen Bartlings, in denen er nicht allein für die Institutionalisierung eines öffentlichen Universitätsherbariums plädierte, sondern gleichermaßen dessen Bedeutsamkeit ausführlich darlegen und rechtfertigen musste, ist das Selbstverständnis, mit dem Grisebach eine solche Aussage tätigte, beachtlich. Innerhalb von 43 Jahren hatte sich offensichtlich nicht nur das Herbarium gegenüber dem botanischen Garten behaupten können; die Forschungsparadigmen hatten sich in solchem Maß verschoben, dass die Relevanz des botanischen Gartens für wissenschaftliche Tätigkeiten sogar angezweifelt wurde. Die hinter dieser Entwicklung stehenden Praktiken und Akteure sowie nicht zuletzt die zentrale Rolle, die den wissenschaftlichen Objekten in diesem Prozess selbst zuteilwurde, standen im Blickpunkt der vorliegenden Arbeit. Die Institutionalisierung und Etablierung des Göttinger Herbariums im Zeitraum von 1832 bis 1852 ist dabei als historische Ethnographie geschrieben worden. Anhand fünf verschiedener Praktiken und ausgewählten Objekten folgend, wurde das Zusammenwirken von Akteuren, Praktiken und Wissensformaten beleuchtet, wobei besonders die materielle Seite der Wissensproduktion herausgestellt wurde.

Voraussetzung für die Herausbildung des Göttinger Herbariums als eigenständige Institution war zunächst die explizite Differenzierung zwischen universitären und privaten Sammlungsbeständen sowie der Wunsch nach Öffnung. Als Universitätsbesitz gehörten die Herbarien aus Sicht des damaligen Privatdozenten und späteren Direktors des botanischen Instituts Bartling zugänglich und nutzbar gemacht und deshalb verfocht er - sogar gegen die Widersprüche seines Kollegen Schraders - die Planung eines öffentlichen Herbariums. Während die Sammlungen der Universität bis 1832 vermengt mit dem Privatherbarium Schraders in dessen Dienstwohnung aufbewahrt wurden, fand mit der Institutionalisierung einhergehend eine Neuausrichtung der Bestände statt. Das Bestreben, die Sammlung für Wissenschaftler*innen und Studierende zu öffnen, führte in der Folge paradoxerweise zunächst sogar zu ihrer Schließung. Denn mit der Gründung des Herbariums gingen eine Spezialisierung und Ausdifferenzierung der Sammlung einher: Über die Nutzbarmachung hinaus strebte Bartling eine Überarbeitung des Bestandes nach damaligen wissenschaftlichen Kriterien und Wertmaßstäben an. Die Präparate sollten auf bestimmte Weise zugerichtet, sortiert und in angemessenen Räumlichkeiten untergebracht werden. Um diese neu formulierten Bedürfnisse und Ansprüche abzusichern und eine verbindliche Infrastruktur zu schaffen, wurden Regularien und Instruktionen verfasst. Diese legten aber nicht nur Kriterien fest, nach denen das vorhandene Material zu behandeln war, sondern forderten gleichzeitig einen Ausbau der Sammlung. Mit dem Anspruch auf eine erhöhte wissenschaftliche Wertigkeit der Herbarbelege

Demonstrationen der kultivierten Pflanzen und der Förderung des wissenschaftlichen

Fortschritts der systematischen Botanik. Vgl. ebd. 
trat der Altbestand des Academischen Museums in seiner Bedeutsamkeit zurück und die Akquirierung neuer Pflanzenexemplare wurde in den Fokus gerückt. Die dabei verfolgten Strategien waren der Ankauf, das Sammeln auf Exkursionen, der Tauschhandel sowie die Schenkung. Dabei handelte es sich natürlich um keine Neuerung an sich, da alle diese Strategien bereits vor der Institutionalisierung wirksam waren. Indem das Herbarium einem enzyklopädischen Sammlungsideal folgend nach Vollständigkeit strebte, wurden allerdings sehr viel gezielter Pflanzenexemplare und Sammlungen akquiriert und zu diesem Zweck vermehrt auch größere Exkursionen unternommen. Indem der neuen Institution ein jährlicher Fonds zur Verfügung gestellt wurde, mussten die anfallenden Kosten auch nicht mehr durch private Mittel getragen werden. Der Erwerb der Sammlungsobjekte oblag dem Vorsteher der Sammlung und dieser wirkte nun weniger als Privatperson, denn als Vertreter einer Institution. Von einem unabhängigen, vollkommen eigenmächtigen Handeln konnte dennoch nicht die Rede sein: Der Sammlungsvorsteher stand regelmäßig unter Rechtfertigungsdruck - ganz besonders, da die zur Verfügung gestellten Gelder zumeist nicht ausreichten und das Herbarium seinen wissenschaftlichen Stellenwert immer wieder behaupten musste.

Der Einsatz der Sammlung in Forschung und Lehre, wie er von Bartling angestrebt wurde und der ihn überhaupt zur Institutionalisierung des Herbariums angetrieben hatte, wurde erst allmählich erreicht. Schließlich waren der Sammlungsaufbau und -ausbau grundlegende Voraussetzungen für eine weiterführende Nutzung. Neben dem zeitintensiven Lehrbetrieb widmeten sich Bartling und der Assistent des Herbariums, Lantzius-Beninga, aus diesem Grund intensiv den Sammlungstätigkeiten. Forschungsarbeit fand für sie in erster Linie im Rahmen der neuen Institution statt. Welche Konsequenz dies für ihr wissenschaftliches Wirken hatte, zeigt sich beispielhaft an ihrer Publikationstätigkeit. Sie nahm in Folge der aufwendigen Bearbeitung stark ab und beschränkte sich thematisch auf systematische Studien oder Florenwerke mit direktem Bezug zur Arbeit am Herbarium. Ein weiterführendes Beforschen der Pflanzenpräparate wurde vielmehr von Grisebach betrieben. Auf systematische Studien aufbauend verfolgte er Fragestellungen der aufkommenden pflanzengeografischen Forschung. Die Verantwortlichkeiten und dienstlichen Verpflichtungen der am Herbarium tätigen Personen standen häufig, das zeigt ein Vergleich der Forschenden, im Konflikt mit persönlichen wissenschaftlichen Interessen. Die Abhängigkeit vom Universitätskuratorium in Hannover verschärfte dieses Spannungsverhältnis. Ein weitestgehend freies und unabhängiges Forschen war den Sammlungsmitarbeiter*innen kaum vergönnt. Darüber hinaus führte die Bindung an die Sammlung dazu, dass neue Forschungsparadigmen und Forschungsfelder wie die Pflanzengeografie zugunsten des Sammlungsaufbaus nur eingeschränkt verfolgt werden konnten. Die Öffnung der Sammlung für Studierende erfolgte ob dieser zeitintensiven Tätigkeit erst 1838. Die Lehre mit den Objekten fand aus diesem Grund weniger 
in der Sammlung selbst statt. Eine Auseinandersetzung mit den Pflanzenexemplaren erfolgte für die Studierenden vielmehr auf Exkursionen und ganz besonders indem Dozierende Belege in die Veranstaltungen mitbrachten.

Da Akteure, Praktiken und Wissensformate in einem ständigen Wechselverhältnis zueinander standen, eröffnete erst eine symmetrische Betrachtungsweise ein tiefergehendes Verständnis dieser zur Herausbildung des Herbariums führenden, vielschichtigen Mechanismen. Der ethnografische Zugang ermöglichte es darüber hinaus, Details zu fokussieren, sie zu beschreiben und immer wieder in die breiteren Zusammenhänge zurückzuverorten. Dieses Spiel von Nähe und Distanz wurde zudem durch die räumliche und zeitliche Eingrenzung des Forschungsgegenstandes begünstigt. Auf diese Weise konnte herausgestellt werden, wie die Herbarbelege als wissenschaftliche Präparate im betrachteten Zeitraum zunehmend Bedeutsamkeit erfuhren. Erst durch diesen Zugewinn war eine Institutionalisierung der Sammlung überhaupt möglich. Dabei kann die Gründung selbstverständlich auch als eine Geschichte einzelner Forschungspersönlichkeiten betrachtet werden. ${ }^{389}$ Doch der vielschichtige Bedeutungswandel, dem die Sammlung und mit ihr die Objekte im Zeitraum von 1832 bis 1852 unterlagen, lässt sich durch ein alleiniges Beleuchten einzelner Akteure kaum ausreichend nachvollziehen. Erst eine multiperspektivische Betrachtungsweise zeigt eindrücklich, wie sich persönliche Interessen, wissenschaftlicher Diskurs und institutionelle Rahmenbedingungen gegenseitig bedingten. So machten sowohl eine Verschiebung wissenschaftlicher Forschungsparadigmen als auch das Konkurrenzprinzip der Georgia Augusta es möglich, dass Bartling als Privatdozent seine Pläne gegen den dienstälteren und ordentlichen Professor Schrader durchsetzen konnte. Damit zeichneten sich auf der einen Seite übergeordnete diskursive Strukturen ab. Auf der anderen Seite wurden aber vor allem auch die Aushandlungen innerhalb des spezifischen Wissensmilieus mit seinen personenund standortgebundenen Voraussetzungen herausgestellt. Im betrachteten Zeitraum trat das Herbarium als ebenbürtige universitäre Sammlung neben den botanischen Garten. Die Botaniker, die diese Entwicklung maßgeblich begleiteten, Schrader, Bartling, Lantzius-Beninga und Grisebach, standen für unterschiedliche wissenschaftliche Schulen. Am Beispiel dieser Forschenden ließen sich wandelnde wissenschaftliche Überzeugungen und damit einhergehende Praktiken deutlich nachzeichnen.

Dabei zeigte sich, dass die in diesen Prozess eingebundenen Herbarbelege nicht allein als materielle Zeugen wissenschaftlichen Erkenntnisinteresses fungieren. Die wissenskonstituierenden Praktiken am Göttinger Herbarium schrieben sich nicht nur in die Objekte ein. Gleichermaßen legten die Präparate selbst einen Umgang mit sich nahe. So wurden die einzelnen Belege zwar an bestimmten Ordnungsprinzipien orientiert erstellt und aufgestellt. Diese

389 Vgl. Füssel 2015b, S. 84; Weber 2011, S. 84. 
spezifische Zurichtung und Anordnung der wissenschaftlichen Objekte ermöglichte es dann aber erst, dass darauf aufbauende Forschungstätigkeiten fruchtbar gemacht werden konnten. Systematische und floristische Studien bedingten die Herausbildung der Pflanzengeografie. Dabei waren einzeln auf Papierbögen fixierte, wissenschaftlich bezeichnete und nach einem in sich einheitlichen Prinzip aufgestellte Pflanzenpräparate die Voraussetzung für einen Vergleich der Pflanzenarten über Landesgrenzen hinweg und damit für Fragen, die auf ihre Verbreitung hin abzielten.

Im Rahmen des Institutionalisierungsprozesses traten darüber hinaus Prinzipien hervor, die zum Teil noch bis in die Gegenwart fortwirken. Das betrifft die bürokratischen Abläufe des Hochschulbetriebs wie beispielsweise die Beantragung von Geldern und Forschungsreisen, aber auch die immer wiederkehrende Aushandlung von wissenschaftlichen Freiheiten und gleichzeitigen Abhängigkeiten sowie nicht zuletzt das Konkurrenzprinzip zwischen den Forschenden als Antriebfeder wissenschaftlicher Erkenntnis. Innerhalb der Sammlung selbst sind solche Kontinuitäten ebenso festzumachen: Bis heute ist das Herbarium in unterschiedliche Abteilungen gegliedert. Die Zurichtung der Belege erfolgt nach sehr ähnlichen Prinzipien und selbstverständlich ist die Sammlung noch immer nach Pflanzenfamilien geordnet. 390 Die Wissenschaftler*innen führen zwar Privatsammlungen, doch in universitären Forschungsprojekten Gesammeltes gilt noch immer als Eigentum der Universität und geht damit stets in die Sammlung über.

Die Beschreibung des Selbstbehauptungsprozesses der Botanik als eigenständige Disziplin durch Ausdifferenzierung und Spezialisierung am Beispiel der Institutionalisierung eines konkreten Sammlungsbestandes stellt dabei nicht nur eine Ergänzung zu den zahlreichen Publikationen über die Naturgeschichte des 17. und 18. Jahrhunderts dar. Gleichermaßen handelt es sich bei der Nutzbarmachung und Öffnung universitärer Sammlungen für eine Fachöffentlichkeit auch um eine parallele Entwicklung zu den Popularisierungsentwicklungen und -tendenzen des 19. Jahrhunderts mit seinen Museumsgründungen und dem zunehmenden Wechselverhältnis zwischen Wissenschaft und verschiedenen Öffentlichkeiten. Mit der Institutionalisierung des Göttinger Herbariums wurde zweifelsohne ein maßgeblicher Schritt zur Herausbildung der Botanik als eigenständige Disziplin getan. Das verdeutlicht nicht zuletzt ein Blick zurück auf den Kontext der von Grisebach 1875 formulierten Einschätzung zur wissenschaftlichen Bedeutsamkeit der an das botanische Institut angegliederten Sammlungen. Denn dem an den Minister in Berlin gerichteten Schreiben gingen zwei Ereignisse voraus: die Gründung eines physiologischen Instituts im Jahr 1873 sowie der Tod Bartlings am 19. November 1875. Die Vakanz der Direktorenstelle nahm Grisebach zum Anlass, „das Verhältnis der vorhandenen Lehrkräfte zu den

390 Heute ist die Hauptsammlung nach dem System von Adolf Engler (1844-1930) aufgestellt. Das Typenherbar ist hingegen alphabetisch sortiert. 
bestehenden botanischen Instituten und die Bedeutung dieser letztern als Lehrmittel, so wie in wissenschaftlicher Beziehung zu erläutern“391. Damit reagierte er gleichfalls auf die Pläne der philosophischen Fakultät, einen neuen Ordinarius der Botanik zu berufen, um die doppelte Professur im Fach zu erhalten - ein Plan, der Grisebach widerstrebte. Das Hinzutreten einer dritten botanischen Einrichtung trieb ihn vielmehr dazu an, nochmals für eine Vertretung durch eine einheitliche Direktion zu plädieren. ${ }^{392}$ Am 1. April 1876 schied Grisebach deshalb schließlich aus der medizinischen Fakultät aus und wechselte unter Übernahme Bartlings Professur zur philosophischen Fakultät. ${ }^{393}$ Damit wurde die Botanik endgültig von der Medizin losgelöst und die an das botanische Institut angegliederten Einrichtungen samt ihrer Sammlungen als formal ebenbürtig behandelt.

391 UniA GÖ, Kur. Pers., 4973, Schreiben Grisebachs an den königlichen Senatsminister und Minister der geistlichen, Unterrichts- und Medizinal-Angelegenheiten Herrn Dr. Falk in Berlin, 11.12.1875.

392 Mit diesem Anliegen trat Grisebach laut seines Schreibens bereits im Januar 1873 an das Ministerium in Berlin heran. Vgl. ebd.

393 Vgl. UniA GÖ, Kur. Pers. 4973, Schreiben des Ministeriums der geistlichen, Unterrichtsund Medizinal-Angelegenheiten an den königlichen Universitätskurator und Geheimen Regierungsrat Herrn Dr. von Warnstedt (Göttingen), Berlin, 26.4.1876. 


\section{Quellen- und Literaturverzeichnis}

I. Archivalische Quellen

\section{Universitätsarchiv Göttingen:}

UniA GÖ, Kur., 5560: Abnahme und Revision der Rechnungen. 1814-1863.

UniA GÖ, Kur., 5579: Erlaubnis zur Benutzung des botanischen Gartens für Privatdozent Dr. Bartling für seine Lehrveranstaltungen. 1829-1836.

UniA GÖ, Kur. 5584: Einrichtung eines Universitätsherbariums und dessen Verwaltung; Reisekostenzuschuss für Prof. Dr. Bartling. 21.06.1830_ 19.05.1852.

UniA GÖ, Kur., 5586: Ankauf der Herbarien Prof. Dr. Schraders und des Gärtners Fischer nach deren Tod. 1837-1837.

UniA GÖ, Kur., 5588: Säcularfeier. 1837-1838.

UniA GÖ, Kur., 5590: Regelung der Vertretung Prof. Dr. Schraders nach dessen Tod. 1836-1837.

UniA GÖ, Kur., 5592: Abnahme und Revision der Rechnungen des Universitätsherbariums. 1834-1867.

UniA GÖ, Kur., 5600: Ankauf der Pflanzensammlung des Dr. Philipp aus Kassel für das Universitätsherbarium. 1846-1846. 
UniA GÖ, Kur., 5603: Zahlungen an den Privatdozenten Dr. Lantzius-Beninga für Hilfsarbeiten im Universitätsherbarium. 25.10.1847-30.10.1847.

UniA GÖ, Kur., 5605: Räume des Universitätsherbariums. 1852-1856.

UniA GÖ, Kur., 5835: Forschungen des Privatdozenten Dr. Lantzius-Beninga über die Flora Ostfrieslands im Rahmen der „Flora Hannoverana“. Enthält auch: Druck der Ergebnisse. 16.01.1849-26.05.1849.

UniA GÖ, Kur., 6912: Ankauf des Meisterschen Hauses zur Erweiterung der Bibliothek und dessen einstweilige Benutzung. 1833-1843.

UniA GÖ, Kur. Pers., 4949: Schrader, Prof. Dr. Heinrich Adolf (Professor der Medizin). 1802-1836.

UniA GÖ, Kur. Pers., 4973: Grisebach, Prof. Dr. August (Professor der Medizin). 1837-1879.

UniA GÖ, Kur. Pers., 5828: Bartling, Prof. Dr. Friedrich Georg (Professor der Naturwissenschaften). 1831-1875.

UniA GÖ, Kur. Pers., 5909: Lantzius-Beninga, Prof. Dr. Boyung Scato (Professor der Naturwissenschaften). 05.09.1846-10.02.1872.

UniA GÖ, Sek., 450.4: Botanischer Garten. Herbarium. 1792-1837.

UniA GÖ, Sek., 528: Das Meister-Heerensche Haus; ,alte Auditorienhäuser“;

Verlegung des Universitäts-Herbarium. 1833-1833.

\section{Staats- und Universitätsbibliothek Göttingen, Handschriftenabteilung:}

Cod. Ms. 1990.24/1: Anatomie und Physiologie der Pflanzen.

Cod. Ms. 1990.24/2: Oeconomische und Forst-Botanik.

Cod. Ms. 2016.12/2: Medicinische Botanik.

2 Cod. Ms. philos. 182: F. G. Bartling.

2 Cod. Ms. philos. 182: A. H. R. Grisebach.

2 Cod. Ms. philos. 182: H. A. Schrader.

4 Cod. Ms. hist. nat. 59 a, Angeb.: Medicinische Botanik.

4 Cod. Ms. hist. nat. 63 d: 13: Lectiones de Botanica [Vorlesungsnachschrift].

4 Cod. Ms. hist. nat. 101 d: Die Einleitung in die Botanik.

4 Cod. Ms. hist. nat. Nachtr. 10: Botanik [Vorlesungsnachschrift].

4 Cod. Ms. hist. nat. Nachtr. 11: Medicinische Botanik [Vorlesungsnachschrift].

8 Cod. Ms. hist. nat. 63 e: Botanik [Vorlesungsnachschrift].

8 Cod. Ms. philos. 13 o: Landwirthschaftliche Botanik. 
Albrecht-von-Haller-Institut für Pflanzenwissenschaften:

PWI.Syst.Geobotan.A1: Dienst-Instruktion für den Direktor, 1838.

PWI.Syst.Geobotan.A19: Div. gedruckte Verordnungen, Verzeichnisse, 1792-1923.

PWI.Syst.Geobotan.HE2: Akzessions-Katalog des Herbariums (und Museums), geführt ab 1876 (mit nachträglicher unvollständiger Zusammenstellung für die Zeit seit 1833). Fortlaufend geführt bis 1918, dann wieder seit 1952; dazwischen nur vereinzelte Angaben.

PWI.Syst.Geobotan.HE15: Verzeichnis von Veröffentlichungen von H. A. Schrader (mit Standnummern in U.B.), in denen neue Arten beschrieben wurden, mit Aufzählung einiger dieser Arten.

PWI.Syst.Geobotan.HV1: Rechnung über Einnahmen und Ausgaben für das Universitäts-Herbarium, 1836/37.

PWI.Syst.Geobotan.HV2: Rechnung über Einnahmen und Ausgaben für das Universitäts-Herbarium, 1837/38.

PWI.Syst.Geobotan.HV3: Rechnung über Einnahmen und Ausgaben für das Universitäts-Herbarium, 1839/40.

PWI.Syst.Geobotan.HV3: Rechnung über Einnahmen und Ausgaben für das Universitäts-Herbarium, 1839/40.

PWI.Syst.Geobotan.HV4: Rechnung über Einnahmen und Ausgaben für das Universitäts-Herbarium, 1840/41.

PWI.Syst.Geobotan.HV5: Rechnung über Einnahmen und Ausgaben für das Universitäts-Herbarium, 1843/44.

PWI.Syst.Geobotan.HV6: Rechnung über Einnahmen und Ausgaben für das Universitäts-Herbarium, 1847/48.

PWI.Syst.Geobotan.HV7: Rechnung über Einnahmen und Ausgaben für das Universitäts-Herbarium, 1848/49.

PWI.Syst.Geobotan.HV8: Rechnung über Einnahmen und Ausgaben für das Universitäts-Herbarium, 1854/55.

PWI.Syst.Geobotan.HV9: Rechnung über Einnahmen und Ausgaben für das Universitäts-Herbarium, 1860/61.

\section{Stadtarchiv Göttingen:}

StadtA GOE, B 100 Nr. 293: Haus-Nr. 293 = Rothestraße 32, 1764-1854.

StadtA GOE, IV A 1: Verzeichnis der öffentlichen und Privat-Lehrer, der Mitglieder der academischen Behörden und der Studierenden auf der GeorgAugust-Universität Göttingen, SS 1822-WS 1823/24, SS 1825, WS 1826/27SS 1828, SS 1829-SS 1894, WS 1899/1900-WS 1908/09, WS 1917/18-SS 1922. 
II. Gedruckte Quellen

Bartling 1820 - Friedrich Gottlieb Bartling: De Littoribus Ac Insulis Maris Liburnici (Dissertatio Inauguralis Geographico-Botanica), Hannover 1820.

Bartling \& Wendland 1824 - Friedrich Gottlieb Bartling \& Heinrich Ludolph Wendland: Beiträge zur Botanik. Erstes Heft, Göttingen 1824.

Bartling \& Wendland 1825 - Friedrich Gottlieb Bartling \& Heinrich Ludolph Wendland: Beiträge zur Botanik. Zweites Heft, Göttingen 1825.

Bartling 1830 - Friedrich Gottlieb Bartling: Ordines Naturales Plantarum, Göttingen 1830.

Bartling 1837 - Friedrich Gottlieb Bartling: Der botanische Garten zu Göttingen im Jahre 1837. Skizze, Göttingen 1837.

Ecklon 1833 - Christian Friedrich Ecklon: Nachricht über die von Ecklon und Zeyher in Südafrika unternommenen Reisen und deren Ausbeute in botanischer Hinsicht. In: Linnaea 8 (1833), S. 390-394.

Ecklon \& Zeyher 1835 - Christian Friedrich Ecklon \& Carl Ludwig Philipp Zeyher: Enumeratio plantarum Africae australis extratropicae Pars. 1, Hamburg 1835.

Georg-August-Universität Göttingen 1830-1852 - Georg-August-Universität Göttingen: Verzeichnis der Vorlesungen, Göttingen 1830-1852, URL: https://t1p.de/Historische-Vorlesungsverzeichnisse (Zugriff: 5.8.2019).

Grisebach 1854 - August Heinrich Rudolf Grisebach: Systematische Bemerkungen über die beiden ersten Pflanzensammlungen Philippi's und Lechler's im südlichen Chile und an der Maghellans-Strasse, Göttingen 1854.

Grisebach 1872a - August Heinrich Rudolf Grisebach: Die Vegetation der Erde nach ihrer klimatischen Anordnung. Ein Abriss der vergleichenden Geographie der Pflanzen, Bd. 1, Leipzig 1872.

Grisebach 1872b - August Heinrich Rudolf Grisebach: Die Vegetation der Erde nach ihrer klimatischen Anordnung. Ein Abriss der vergleichenden Geographie der Pflanzen, Bd. 2, Leipzig 1872.

Grisebach 1880 - August Heinrich Rudolf Grisebach: Über die Vegetationslinien des Nordwestlichen Deutschlands. Ein Beitrag zur Geographie der Pflanzen (1847). In: Eduard Grisebach (Hg.): Gesammelte Abhandlungen und kleinere Schriften zur Pflanzengeographie von A. Grisebach, Leipzig 1880, 136-216.

Hirsching 1792 - Friedrich Karl Gottlob Hirsching (Hg.): Nachrichten von sehenswürdigen Gemälde- und Kupferstichsammlungen, Münz-, Gemmen-, Kunst- und Naturalienkabineten, Sammlungen von Modellen, Maschinen, physikalischen und mathematischen Instrumenten, anatomischen Präparaten und botanischen Gärten in Deutschland, nach alphabetischer Ordnung der Oerter, Bd. 6, Erlangen 1792. 
Lantzius-Beninga 1844 - Scato Lantzius-Beninga: De evolutione sporidiorum in capsulis muscorum (Dissertatio Inauguralis), Göttingen 1844.

Lantzius-Beninga 1847 - Scato Lantzius-Beninga: Beiträge zur Kenntnis des inneren Baues der ausgewachsenen Mooskapsel, insbesondere des Peristomes. In: Botanische Zeitung 5 (1847), S. 17-22.

Lantzius-Beninga 1849 - Scato Lantzius-Beninga: Beiträge zur Kenntniss der Flora Ostfriesland's, Göttingen 1849.

Linné 1751 - Carl von Linné: Philosophia botanica in qua explicantur fundamenta botanica cum definitionibus partium, exemplis terminorum, observationibus rariorum, adjectis figuris aeneis, Stockholm 1751.

Meyer 1836 - Georg Friedrich Wilhem Meyer: Chloris Hanoverana oder nach den natürlichen Familien geordnete Übersicht der im Königreiche Hannover wildwachsenden sichtbar blühenden Gewächse und Farn, Göttingen 1836.

o. A. 1838 - o. A.: Reliquiae Schraderianae. In: Linnaea 12 (1838), S. 354-476.

III. Verwendete Literatur

Alberti 2005 - Samuel J. M. M. Alberti: Owning and collecting natural objects in nineteenth-century Britain. In: Marco Beretta (Hg.): From Private to Public. Natural Collections and Museums (Uppsala Studies in History of Science, Bd. 32 \& European Studies in Science History and the Arts, Bd. 5.), Sagamore Beach 2005, S. 141-154.

Andraschke \& Ruisinger 2007 - Udo Andraschke \& Marion Maria Ruisinger: Die Sammlungen der Universität Erlangen-Nürnberg (Ausst.-Kat., Ausgepackt. Die Sammlungen der Universität Erlangen-Nürnberg, 20.5-29.7.2007, Stadtmuseum Erlangen), Nürnberg 2007.

Ash 2000 - Mitchell G. Ash: Raume des Wissens - was und wo sind sie?

Einleitung in das Thema. In: Berichte zur Wissenschaftsgeschichte 23 (2000), S. 235-242.

Ash 2007 - Mitchell G. Ash: Wissenschaft(en) und Öffentlichkeit(en) als Ressourcen füreinander. In: Sybilla Nikolow \& Arne Schirrmacher (Hg.): Wissenschaft und Öffentlichkeit als Ressourcen füreinander. Studien zur Wissenschaftsgeschichte im 20. Jahrhundert, Frankfurt am Main 2007, S. 349-362.

Bachmann-Medick 2010 - Doris Bachmann-Medick: ,Cultural Turns', Version:

1.0. In: Docupedia-Zeitgeschichte, 29.3.2010, URL: http://docupedia.de/zg/Cultural_Turns (Zugriff: 4.8.2019).

Becker \& Clark 2001 - Peter Becker \& William Clark: Little Tools of Knowledge. Historical Essays on Academic and Bureaucratic Practices (Social history, popular culture, and politics in Germany), Ann Arbor 2001. 
Beretta 2005 - Marco Beretta: Preface. In: Marco Beretta (Hg.): From Private to Public. Natural Collections and Museums (Uppsala Studies in History of Science, Bd. 32 \& European Studies in Science History and the Arts, Bd. 5.), Sagamore Beach 2005, S. vii-ix.

Bergsträsser \& Markert 2018 - Linnéa Bergsträsser \& Michael Markert: Präparate im Unterricht. Zur schulischen Relevanz der Firma Schlüter und ihres Lehrmittelangebots in Vergangenheit und Gegenwart. In: Vernate 37 (2018), S. 5-21.

Bleichmar \& Mancall 2011 - Daniela Bleichmar \& Peter C. Mancall: Collecting Across Cultures. Material Exchanges in the Early Modern Atlantic World, Philadelphia 2011.

Boie et al. 2009 - Jenni Boie, Antonia Davidovic-Walther, Carsten Drieschner, Michaela Fenske, Silke Göttsch, Sabine Imeri, Wolfgang Kaschuba, Lioba Keller-Drescher \& Franka Schneider: Volkskundliches Wissen und gesellschaftlicher Wissenstransfer. Zur Produktion kultureller Wissensformate im 20. Jahrhundert (DFG-Forschungsverbund). In: Michael Simon et al. (Hg.): Bilder. Bücher. Bytes. Zur Medialität des Alltags (Mainzer Beiträge zur Kulturanthropologie/Volkskunde, Bd. 3), Münster, New York \& Berlin 2009, S. 183-199.

Bonneuil 2002 - Christophe Bonneuil: The manufacture of species. Kew Gardens, the Empire, and the standardisation of taxonomic practices in late nineteenth-century botany. In: Marie-Noëlle Bourgouet et al. (Hg.): Instruments, Travel and Science. Itineraries of precision from the seventeenth to the twentieth century (Routledge Studies in the History of Science, Technology and Medicine, Bd. 16), London \& New York 2002, S. 189-215.

Bourguet 2002 - Marie-Noelle Bourguet: Landscape with numbers. Natural history, travel and instruments in the late eighteenth and early nineteenth century. In: Marie-Noelle Bourguet et al. (Hg.): Instruments, Travel and Science. Itineraries of precision from the seventeenth to the twentieth century, London \& New York 2002, S. 96-125.

Bourguet 2005 - Marie-Noelle Bourguet: Measurable Difference. Botany, Climate, and the Gardener's Thermometer in Eighteenth-Century France. In: Londa Schiebinger \& Claudia Swan (Hg.): Colonial Botany. Science, Commerce, and Politics in the Early Modern World, Philadelphia 2005, S. $270-286$.

Bredekamp et al. 2000 - Horst Bredekamp, Jochen Brüning \& Cornelia Weber (Hg.): Theater der Natur und Kunst. Essays (Ausst.-Kat., Theatrum naturae et artis - Theater der Natur und Kunst. Wunderkammern des Wissens, 10.12.2000-4.3.2001, Martin-Gropius-Bau Berlin), Berlin 2000. 
Bruning 2011 - Jens Bruning: Vorlesungsverzeichnisse. In: Ulrich Rasche (Hg.): Quellen zur frühneuzeitlichen Universitätsgeschichte. Typen, Bestände, Forschungsperspektiven (Wolfenbütteler Forschungen, Herausgegeben von der Herzog August Bibliothek, Bd. 128), Wiesbaden 2011, S. 269-292.

Charmantier \& Müller-Wille 2014 - Isabelle Charmantier \& Staffan Müller-Wille: Carl Linnaeus's botanical paper slips (1767-1773). In: Intellectual History Review 24/2 (2014), S. 215-238, DOI: 10.1080/17496977.2014.914643 (Zugriff: 24.7.2019)

Cunningham 1996 - Andrew Cunningham: The culture of gardens. In: Nicholas Jardine et al. (Hg.): Cultures of natural history, Cambridge \& New York 1996, S. 38-56.

Dahlenburg 2011 - Birgit Dahlenburg (Hg.): Wissen sammeln. Die digitalisierten Schätze der Universität Greifswald. Sammlungsobjekte der Botanik, Zoologie sowie Ur- und Frühgeschichte, Greifswald 2011.

Daston 1999 - Lorraine Daston: The Moralized Objectivities of Science. In: Wolfgang Carl \& Lorraine Daston (Hg.): Wahrheit und Geschichte. Ein Kolloquium zu Ehren des 60. Geburtstages von Lorenz Krüger (Abhandlungen der Akademie der Wissenschaften in Göttingen. Philologischhistorische Klasse, Dritte Folge, Nr. 231), Göttingen 1999, S. 78-100.

Daston 2001 - Lorraine Daston: Scientific Objectivity with and without Words. In: Peter Becker \& William Clark: Little Tools of Knowledge. Historical Essays on Academic and Bureaucratic Practices (Social history, popular culture, and politics in Germany), Ann Arbor 2001, S. 259-284.

Daston 2004 - Lorraine Daston: Type Specimens and Scientific Memory. In: Critical Inquiry 31/1 (2004), S. 153-182.

Daston \& Galison 2007 - Lorraine Daston \& Peter Galison: Objektivität, Frankfurt am Main 2007 (Engl. Orig.: Objectivity, New York 2007).

Daston 2011 - Lorraine Daston: The Empire of Observation, 1600-1800. In: Lorraine Daston \& Elizabeth Lunbeck (Hg.): Histories of Scientific Observation, Chicago \& London 2011, S. 81-113.

Daum 1998 - Andreas Daum: Naturwissenschaften und Öffentlichkeit in der deutschen Gesellschaft. Zu den Anfängen einer Populärwissenschaft nach der Revolution von 1848. In: Historische Zeitschrift 267 (1998), S. 57-90.

Daum 2002 - Andreas Daum: Science, Politics, and Religion. Humboldtian Thinking and the Transformations of Civil Society in Germany, 1830-1870. In: Osiris 17 (2002), S. 107-140. 
Dauser et al. 2008 - Regina Dauser, Stefan Hächler, Michael Kempe, Franz Mauelshagen \& Martin Stuber: Einleitung. In: Regina Dauser et al. (Hg.): Wissen im Netz. Botanik und Pflanzentransfer in europäischen Korrespondenznetzen des 18. Jahrhunderts (Institut für Europäische Kulturgeschichte der Universität Augsburg, Colloquia Augustana, Bd. 24), Berlin 2008, S. 9-28.

Davidovic-Walther et al. 2009 - Antonia Davidovic-Walther, Michaela Fenske \& Lioba Keller-Drescher: Akteure und Praktiken. Explorationen volkskundlicher Wissensproduktion. In: Berliner Blätter. Ethnographische und ethnologische Beiträge 50 (2009), S. 6-14.

Desmond 1995 - Ray Desmond: Kew. The History of the Royal Botanic Gardens, London 1995.

Dietzsch et al. 2009 - Ina Dietzsch, Sabine Imeri, Wolfgang Kaschuba, Cornelia Kühn \& Leonore Scholze-Irrlitz: Einleitung. In: Ina Dietzsch et al. (Hg.): Horizonte ethnografischen Wissens. Eine Bestandsaufnahme, Köln, Weimar \& Wien 2009, S. 7-15.

Dolezel et al. 2018 - Eva Dolezel, Rainer Godel, Andreas Pečar \& Holger Zaunstöck: Ordnen - Vernetzen - Vermitteln. Kunst- und Naturalienkammern der Frühen Neuzeit als Lehr- und Lernorte. In: Eva Dolezel et al. (Hg.): Ordnen - Vernetzen - Vermitteln. Kunst- und Naturalienkammern der Frühen Neuzeit als Lehr- und Lernorte (Acta Historica Leopoldina 70), Stuttgart 2018, S. 7-18.

Eggert \& Samida 2016 - Manfred K. H. Eggert \& Stefanie Samida: Menschen und Dinge. Anmerkungen zum Materialitätsdiskurs. In: Herbert Kalthoff et al. (Hg.): Materialität. Herausforderungen für die Sozial- und Kulturwissenschaften, Paderborn 2016, S. 123-140.

Elias et al. 2014 - Friederike Elias, Albrecht Franz, Henning Murmann \& Ulrich Wilhelm Weiser: Hinführung zum Thema und Zusammenfassung der Beiträge. In: Friederike Elias et al. (Hg.): Praxeologie. Beiträge zur interdisziplinären Reichweite praxistheoretischer Ansätze in den Geistes- und Sozialwissenschaften (Materiale Textkulturen, Schriftenreihe des Sonderforschungsbereichs 933, Bd. 3), Berlin \& Boston 2014, S. 3-12.

Fenske 2006 - Michaela Fenske: Mikro, Makro, Agency. Historische Ethnografie als kulturanthropologische Praxis. In: Zeitschrift für Volkskunde 102 (2006), S. 151-177.

Fenske 2013 - Michaela Fenske: „Historische Ethnografie“ - Ein Modus des Denkens, Forschens und Darstellens. In: Reinhard Johler et al. (Hg.): Kultur_Kultur. Denken. Forschen. Darstellen (38. Kongress der Deutschen Gesellschaft für Volkskunde in Tübingen vom 21. bis 24. September 2011), Münster et al. 2013, S. 204-205. 
Ford 2014 - Lisa L. Ford: From Plant to Page. Aesthetics and Objectivity in a Nineteenth-Century Book of Trees. In: Pamela H. Smith et al. (Hg.): Ways of making and knowing. The material culture of empirical knowledge, Ann Arbor 2014, S. 221-242.

Förschler \& Mariss 2017 - Silke Förschler \& Anne Mariss: Die frühneuzeitliche Naturgeschichte und ihre Verfahrensweisen. In: Silke Förschler \& Anne Mariss (Hg.): Akteure, Tiere, Dinge. Verfahrensweisen der Naturgeschichte in der Frühen Neuzeit, Köln et al. 2017, S. 7-26.

François 2015 - Etienne François: Das Zeitalter der $>$ Haupt- und Residenzstädte <. In: Bénédicte Savoy (Hg.): Tempel der Kunst. Die Geburt des öffentlichen Museums in Deutschland 1701-1815, Köln, Weimar \& Wien 2015, S. 46-57.

Freist 2015 - Dagmar Freist: Diskurse - Körper - Artefakte. Historische Praxeologie in der Frühneuzeitforschung - eine Annäherung. In: Dagmar Freist (Hg.): Diskurse - Körper - Artefakte. Historische Praxeologie in der Frühneuzeitforschung, Bielefeld 2015, S. 9-30.

Funk 2003 - Vicki Ann Funk: 100 Uses for an Herbarium. Well at least 72. In: American Society of Plant Taxonomists Newsletter 17/2 (2003), S. 17-19.

Füssel 2015a - Marian Füssel: Die Universität der Dinge. Zur universitätshistorischen Verortung des Sammlungswesens. In: Georg-August-Universität Göttingen (Hg.): Dinge des Wissens. Die Sammlungen, Museen und Gärten der Universität Göttingen (Ausst.-Kat., Dinge des Wissens, 6.2.-10.7.2012, Göttingen), Göttingen 2015, S. 52-59.

Füssel 2015b - Marian Füssel: Lehre ohne Forschung? Zu den Praktiken des Wissens an der Universität der Frühen Neuzeit. In: Martin Kintzinger \& Sita Steckel unter Mitarbeit von Julia Crispin (Hg.): Akademische Wissenskulturen. Praktiken des Lehrens und Forschens vom Mittelalter bis zur Moderne (Veröffentlichungen der Gesellschaft für Universitäts- und Wissenschaftsgeschichte, Bd. 13), Basel 2015, S. 59-87.

Gaskell 2017 - Ivan Gaskell: Everything or nothing? What do University Museums know? (The Nineteenth Horst Gerson Lecture, held in memory of Horst Gerson in the aula of the University of Groningen on the 6th of October 2017), Groningen 2017, S. 5-41.

Georg-August-Universität Göttingen 2015 - Georg-August-Universität Göttingen (Hg.): Dinge des Wissens. Die Sammlungen, Museen und Gärten der Universität Göttingen (Ausst.-Kat., Dinge des Wissens, 6.2-10.7.2012, Göttingen), Göttingen 2015.

Haasis \& Rieske 2015 - Lukas Haasis \& Constantin Rieske: Historische Praxeologie. Zur Einführung. In: Lukas Haasis \& Constantin Rieske (Hg.): Historische Praxeologie. Dimensionen vergangenen Handelns, Paderborn 2015, S. 7-54. 
Hahn 2016 - Hans Peter Hahn: Die Unsichtbarkeit der Dinge. Über zwei Perspektiven zu materieller Kultur in den Humanities. In: Herbert Kalthoff et al. (Hg.): Materialität. Herausforderungen für die Sozial- und Kulturwissenschaften, Paderborn 2016, S. 45-62.

Hahn 2018 - Hans Peter Hahn: Vom Sammeln und Sehen. Episteme des Materiellen im Kontext der Wissenschaften. In: Vera Hierholzer (Hg.): Wertsachen. Die Sammlungen der Johannes Gutenberg-Universität Mainz, Göttingen 2018, S. 18-23.

Hennig \& Andraschke 2010 - Jochen Hennig \& Udo Andraschke (Hg.): Weltwissen. 300 Jahre Wissenschaften in Berlin (Ausst.-Kat., Weltwissen 300 Jahre Wissenschaften in Berlin, 24.9.2010-9.1.2011, Martin-Gropius-Bau Berlin), München 2010.

Hierholzer 2018 - Vera Hierholzer (Hg.): Wertsachen. Die Sammlungen der Johannes Gutenberg-Universität Mainz, Göttingen 2018.

Hurka \& Neuffer 2011 - Herbert Hurka \& Barbara Neuffer: Geschichte und Bedeutung von Herbarien. In: Osnabrücker Naturwissenschaftliche Mitteilungen 37 (2011), S. 141-160.

Imeri \& Schneider 2013 - Sabine Imeri \& Franka Schneider: Historische Ethnografie als reflexiver Forschungsmodus. In: Reinhard Johler et al. (Hg.): Kultur_Kultur. Denken. Forschen. Darstellen (38. Kongress der Deutschen Gesellschaft für Volkskunde in Tübingen vom 21. bis 24. September 2011), Münster et al. 2013, S. 213-224.

Isphording 2008 - Eduard Isphording: Kräuter und Blumen. Kommentiertes Bestandsverzeichnis der botanischen Bücher bis 1850 in der Bibliothek des Germanischen Nationalmuseums Nürnberg, Nürnberg 2008.

Jahn 1998 - Ilse Jahn (Hg.): Geschichte der Biologie. Theorien, Methoden, Institutionen, Kurzbiographien (3., neubearbeitete und erweiterte Auflage), Jena et al. 1998.

Joost 2001 - Ulrich Joost: Vorlesungsmanuskript und Vorlesungsnachschrift als editorisches Problem, und etwas von Lichtenbergs Vorlesungen. In: Robert Seidel (Hg.): Wissen und Wissensvermittlung im 18. Jahrhundert. Beiträge zur Sozialgeschichte der Naturwissenschaften zur Zeit der Aufklärung (Cardanus, Jahrbuch für Wissenschaftsgeschichte, Bd.1), Heidelberg 2001, S. 33-70.

Knecht 2013 - Michi Knecht: Nach Writing Culture, mit Actor-Network. Ethnografie/Praxeografie in der Wissenschafts-, Medizin- und Technikforschung. In: Sabine Hess et al. (Hg.): Europäisch-ethnologisches Forschen. Neue Methoden und Konzepte, Berlin 2013, S. 79-106.

König 2004 - Gudrun M. König: Stacheldraht. Die Analyse materieller Kultur und das Prinzip der Dingbedeutsamkeit. In: Österreichische Zeitschrift für Geschichtswissenschaften 15/4 (2004), S. 50-72. 
König \& Papierz 2013 - Gudrun M. König \& Zuzanna Papierz: Plädoyer für eine qualitative Dinganalyse. In: Sabine Hess et al. (Hg.): Europäischethnologisches Forschen. Neue Methoden und Konzepte, Berlin 2013, S. 283-307.

Knoll 2005 - Joachim Knoll: Die Wendlands - gärtnerische Tüchtigkeit und wissenschaftlicher Anspruch. In: Bericht der Naturhistorischen Gesellschaft Hannover 147 (2005), S. 80-112.

Kretschmann 2006 - Carsten Kretschmann: Räume öffnen sich. Naturhistorische Museen im Deutschland des 19. Jahrhunderts (Wissenskultur und Gesellschaftlicher Wandel., Herausgegeben vom Forschungskolleg 435 der Deutschen Forschungsgemeinschaft »Wissenskultur und gesellschaftlicher Wandek, Bd. 12), Berlin 2006.

Laird \& Bridgman 2014 - Mark Laird \& Karen Bridgman: American Roots. Techniques of Plant Transportation and Cultivation in the Early Atlantic World. In: Pamela H. Smith et al. (Hg.): Ways of Making and Knowing. The Material Culture of Empirical Knowledge, Ann Arbor 2014, S. 164-193.

Larsen 1996 - Anne Larsen: Equipment for the field. In: Nicholas Jardine et al. (Hg.): Cultures of natural history, Cambridge 1996, S. 358-377.

Latour 1997 - Bruno Latour: Der Pedologenfaden von Boa Vista. Eine photophilosophische Montage. In: Hans-Jörg Rheinberger et al. (Hg.): Räume des Wissens. Repräsentation, Codierung, Spur, Berlin 1997, S. 213-263.

Latour 2014 - Bruno Latour: Ist Wissen ein Existenzmodus? In: Anke te Heesen \& Margarete Vöhringer (Hg.): Wissenschaft im Museum - Ausstellung im Labor (LiteraturForschung, Bd. 20), Berlin 2014, S. 136-173.

Lehmann 2016 - Ann-Sophie Lehmann: Objektstunden. Vom Materialwissen zur Materialbildung. In: Herbert Kalthoff et al. (Hg.): Materialität. Herausforderungen für die Sozial- und Kulturwissenschaften, Paderborn 2016, S. 171-193.

Lefèvre 2016 - Wolfgang Lefèvre: 'Das Ende der Naturgeschichte' neu verhandelt. Historisch genealogische oder epigenetische Neukonzeption der Natur?, Berlin 2016, URL: http:/ / hdl.handle.net/11858/00-001M-0000-002A-8079-3 (Zugriff: 6.8.2019)

Macho 2000 - Thomas Macho: Sammeln in chronologischer Perspektive. In: Horst Bredekamp et al. (Hg.): Theater der Natur und Kunst. Essays (Ausst.Kat., Theatrum naturae et artis - Theater der Natur und Kunst. Wunderkammern des Wissens, 10.12.2000-4.3.2001, Martin-Gropius-Bau Berlin), Berlin 2000, S. 63-74.

Mägdefrau 2013 - Karl Mägdefrau: Die Verbreitung der Pflanzen. In: Karl Mägdefrau: Geschichte der Botanik. Leben und Leistung großer Forscher (2., Auflage 1992, Unveränderter Nachdruck), Heidelberg 2013, S. 117-134. 
Mariss 2015 - Anne Mariss: Für's Kabinett. Mineraliensammeln als wissenschaftliche Praxis im 18. Jahrhundert. In: Lukas Haasis \& Constantin Rieske (Hg.): Historische Praxeologie. Dimensionen vergangenen Handelns, Paderborn 2015, S. 89-104.

Mariss 2018 - Anne Mariss: Kunst- und Naturalienkammern in Professorenhaushalten: Polyvalente Wissensräume an der Schnittstelle zwischen Gelehrsamkeit und Geselligkeit. In: Eva Dolezel et al. (Hg.): Ordnen - Vernetzen Vermitteln. Kunst- und Naturalienkammern der Frühen Neuzeit als Lehrund Lernorte (Acta Historica Leopoldina 70), Stuttgart 2018, S. 205-230.

Markert 2017 - Michael Markert: »Die lebende Pflanze [...] ist das beste Anschauungsmittel«. Zur Didaktik der Botanik um 1900. In: Angela Nikolai \& Sabine Thümmler: Form Follows Flower. Moritz Meurer, Karl Blossfeldt \& Co., Berlin 2017, S. 85-93.

Müller \& Victor 2016 - Elisabeth Müller \& Kristin Victor (Hg.): Blatt auf Blatt. Einzigartige Geschichten aus dem Herbarium Haussknecht (Laborberichte, Bd. 10), Weimar 2016.

Müller-Wille 1999 - Staffan Müller-Wille: Botanik und weltweiter Handel. Zur Begründung eines Natürlichen Systems der Pflanzen durch Carl von Linné (1707-78) (Studien zur Theorie der Biologie, Bd. 3), Berlin 1999.

Müller-Wille 2001 - Staffan Müller-Wille: Carl von Linnés Herbarschrank. Zur epistemischen Funktion eines Sammlungsmöbels. In: Anke te Heesen \& Emma C. Spary (Hg.): Sammeln als Wissens. Das Sammeln und seine wissenschafts-geschichtliche Bedeutung, Göttingen 2001, S. 22-38.

Müller-Wille 2005 - Staffan Müller-Wille: Walnuts at Hudson Bay, Coral Reefs in Gotland. The Colonialism of Linnaean Botany. In: Londa Schiebinger \& Claudia Swan (Hg.): Colonial Botany. Science, Commerce, and Politics in the Early Modern World, Pennsylvania 2005, S. 34-48.

Müller-Wille 2008 - Staffan Müller-Wille: Botanischer Tausch und Ökonomie der Natur. In: Regina Dauser et al. (Hg.): Wissen im Netz. Botanik und Pflanzentransfer in europäischen Korrespondenznetzen des 18. Jahrhunderts, Berlin 2008, S. 79-89.

Müller-Wille \& Charmantier 2012 - Staffan Müller-Wille \& Isabelle Charmantier: Lists as Research Technologies. In: Isis 103/4 (2012), S. 743-752.

Müller-Wille 2017a - Staffan Müller-Wille: Verfahrensweisen der Naturgeschichte nach Linné. In: Silke Förschler \& Anne Mariss (Hg.): Akteure, Tiere, Dinge. Verfahrensweisen der Naturgeschichte in der Frühen Neuzeit, Köln, Weimar \& Wien 2017, S. 109-124.

Müller-Wille 2017b - Staffan Müller-Wille: Names and Numbers. "Data" in Classical Natural History, 1758-1859. In: OSIRIS 32 (2017), S. 109-128. 
Napolitani 2018 - Maddalena Napolitani: The Wonder of Mineralogy. Teaching and Collecting Practices within the Cabinet of Balthazar Georges Sage between Science and the Marvellous (1783-1794). In: Eva Dolezel et al. (Hg.): Ordnen - Vernetzen - Vermitteln. Kunst- und Naturalienkammern der Frühen Neuzeit als Lehr- und Lernorte (Acta Historica Leopoldina 70), Stuttgart 2018, S. 95-118.

Nawa 2010a - Christine Nawa: Sammeln für die Wissenschaft? Das Academische Museum Göttingen (1773-1840), Göttingen 2010, URL: http:/ hdl.handle.net/11858/00-1735-0000-000D-F1CC-C (Zugriff: 30.7.2019).

Nawa 2010b - Christina Nawa: Zum «öffentlichen Gebrauche» bestimmt. Das Academische Museum Göttingen. In: Göttinger Jahrbuch 58 (2010), S. 23-62.

Nikolow \& Schirrmacher 2007 - Sybilla Nikolow \& Arne Schirrmacher (Hg.): Wissenschaft und Öffentlichkeit als Ressourcen füreinander. Studien zur Wissenschaftsgeschichte im 20. Jahrhundert, Frankfurt am Main 2007.

Nissen 1966 - Claus Nissen: Die botanische Buchillustration. Ihre Geschichte und Bibliographie (Durchgesehener und verbesserter Abdruck der zweibändigen Erstauflage ergänzt durch ein Supplement), Stuttgart 1966.

Niewöhner et al. 2012 - Jörg Niewöhner, Estrid Sørensen \& Stefan Beck: Science and Technology Studies - Wissenschafts- und Technikforschung aus sozialund kulturanthropologischer Perspektive. In: Stefan Beck et al. (Hg.): Science and Technology Studies. Eine sozialanthropologische Einführung, Bielefeld 2012, S. 9-48.

Nualart et al. 2017 - Neus Nualart, Neus Ibáñez, Ignasi Soriano \& Jordi López-Pujol: Assessing the Relevance of Herbarium Collections as Tools for Conservation Biology. In: The Botanical Review 83/3 (2017), S. 303-325.

Nyhart 2002 - Lynn K. Nyhart: Teaching Community Via Biology in LateNineteenth-Century Germany. In: Osiris 17 (2002), S. 141-170.

Oberdiek 2002 - Alfred Oberdiek: Göttinger Universitätsbauten. Die Baugeschichte der Georg-August-Universität (2., überarb. und erw. Aufl.), Göttingen 2012.

Outram 1996 - Dorinda Outram: New spaces in natural history. In: Nicholas Jardine et al. (Hg.): Cultures of natural history, Cambridge 1996, S. 249-265.

Panke-Kochinke 1993 - Birgit Panke-Kochinke: Göttinger Professorenfamilien. Strukturmerkmale weiblichen Lebenszusammenhangs im 18. und 19. Jahrhundert (Forum Frauengeschichte, Bd. 4), Pfaffenweiler 1993.

Paulsen 1965 - Friedrich Paulsen: Geschichte des gelehrten Unterrichts auf den deutschen Schulen und Universitäten vom Ausgang des Mittelalters bis zur Gegenwart (Dritte, erweiterte Auflage, Bd. 2, Der gelehrte Unterricht im Zeichen des Neuhumanismus 1740-1892), Berlin 1965. 
Pelzer 2002 - Marten Pelzer: Landwirtschaftliche Vereine in Nordwestdeutschland. Das Beispiel Badbergen. Eine Mikrostudie zur Vereins- und Agrargeschichte im 19. und frühen 20. Jahrhundert (Quellen und Studien zur Regionalgeschichte Niedersachsens, Bd. 8), Cloppenburg 2002.

Pelzer 2004 - Marten Pelzer: ,,Was die Schule für das heranwachsende Geschlecht ist, das ist der landwirtschaftliche Verein für die älteren Landwirte ..." Bildungsanspruch und -wirklichkeit landwirtschaftlicher Vereine im 19. Jahrhundert. In: Zeitschrift für Agrargeschichte und Agrarsoziologie 52/2 (2004), S. 41-58.

Phillips 2012 - Denise Phillips: Acolytes of Nature. Defining Natural Science in Germany, 1170-1850, Chicago \& London 2012.

Plesker 2015 - Nadine Plesker: Das Königlich Academische Museum in Göttingen. In: Bénédicte Savoy (Hg.): Tempel der Kunst. Die Geburt des öffentlichen Museums in Deutschland 1701-1815, Köln, Weimar \& Wien 2015, S. 413-442.

Pomian 1994 - Krzysztof Pomian: Sammlungen - eine historische Typologie. In: Andreas Grote (Hg.): Macrocosmos in Microcosmo. Die Welt in der Stube. Zur Geschichte des Sammelns 1450 bis 1800, Wiesbaden 1994, S. 107-126.

Pomian 1998 - Krzysztof Pomian: Der Ursprung des Museums. Vom Sammeln, Berlin 1998.

Reckwitz 2003 - Andreas Reckwitz: Grundelemente einer Theorie sozialer Praktiken. Eine sozialtheoretische Perspektive. In: Zeitschrift für Soziologie 32/4 (2003), S. 282-301.

Reckwitz 2014 - Andreas Reckwitz: Die Materialisierung der Kultur. In:

Friederike Elias et al. (Hg.): Praxeologie. Beiträge zur interdisziplinären Reichweite praxistheoretischer Ansätze in den Geistes- und Sozialwissenschaften (Materiale Textkulturen, Schriftenreihe des Sonderforschungsbereichs 933, Bd. 3), Berlin \& Boston 2014, S. 13-25.

Rieppel 2016 - Lukas Rieppel: Museums and Botanical Gardens. In: Bernard Lightman (Hg.): A Companion to the History of Science, Chichester 2016, S. 238-251.

Saada 2008 - Anne Saada: Die Universität Göttingen. Traditionen und Innovationen gelehrter Praktiken. In: Hans Erich Bödeker et al. (Hg.): Die Wissenschaft vom Menschen in Göttingen um 1800. Wissenschaftliche Praktiken, institutionelle Geographie, europäische Netzwerke (Veröffentlichungen des Max-Planck-Instituts für Geschichte, Bd. 237), Göttingen 2008, S. 23-46.

Sarasin 2011 - Philipp Sarasin: Was ist Wissensgeschichte? In: Internationales Archiv für Sozialgeschichte der deutschen Literatur (IASL) 36/1 (2011), S. 159-172, DOI: https://doi.org/10.1515/iasl.2011.010 (Zugriff: 22.7.2019). 
Savoy 2015 - Bénédicte Savoy: Zum Öffentlichkeitscharakter deutscher Museen im 18. Jahrhundert. In: Bénédicte Savoy (Hg.): Tempel der Kunst. Die Geburt des öffentlichen Museums in Deutschland 1701-1815, Köln, Weimar \& Wien 2015, S. 13-45.

Scheidegger 2017 - Tobias Scheidegger: »Petite Science«. Außeruniversitäre Naturforschung in der Schweiz um 1900, Göttingen 2017.

Schiebinger 2004 - Londa Schiebinger: Plants and Empire. Colonial Bioprospecting in the Atlantic World, Cambridge, Massachusetts \& London/England 2004.

Schiebinger \& Swan 2005 - Londa Schiebinger \& Claudia Swan: Introduction. In: Londa Schiebinger \& Claudia Swan (Hg.): Colonial Botany. Science, Commerce, and Politics in the Early Modern World, Pennsylvania 2005, S. 1-16.

Schirrmacher 2008 - Arne Schirrmacher: Nach der Popularisierung. Zur Relation von Wissenschaft und Öffentlichkeit im 20. Jahrhundert. In: Geschichte und Gesellschaft 34 (2008), S. 73-95.

Schlumbohm 2016 - Jürgen Schlumbohm: Markt und Monopol. Konkurrenz als Motor der Universität Göttingen im 18. Jahrhundert. In: Elizabeth Harding (Hg.): Kalkulierte Gelehrsamkeit. Zur Ökonomisierung der Universitäten im 18. Jahrhundert, Wiesbaden 2016, S. 233-256.

Siemer 2004 - Stefan Siemer: Geselligkeit und Methode. Naturgeschichtliches Sammeln im 18. Jahrhundert, Mainz 2004.

Smith et al. 2014 - Pamela H. Smith, Amy R. W. Meyers \& Harold J. Cook (Hg.): Ways of Making and Knowing. The Material Culture of Empirical Knowledge (The Bard Graduate Center cultural histories of the material world), Ann Arbor 2014.

Stafleu 1987 - Frans A. Stafleu: Die Geschichte der Herbarien. In: Bot. Jahrb. Syst. 108 (1987), S. 155-166.

Steinke 2008 - Hubert Steinke: Gelehrte - Liebhaber - Ökonomen. Typen botanischer Briefwechsel im 18. Jahrhundert. In: Regina Dauser et al. (Hg.): Wissen im Netz. Botanik und Pflanzentransfer in europäischen Korrespondenznetzen des 18. Jahrhunderts (Institut für Europäische Kulturgeschichte der Universität Augsburg, Colloquia Augustana, Bd. 24), Berlin 2008, S. 135-147.

Stelter 2018 - Marcus Stelter: Möglichkeiten und Grenzen des Erwerbs und der Vermittlung von Wissen durch Schenkungen. In: Eva Dolezel et al. (Hg.): Ordnen - Vernetzen - Vermitteln. Kunst- und Naturalienkammern der Frühen Neuzeit als Lehr- und Lernorte (Acta Historica Leopoldina 70), Stuttgart 2018, S. 179-204.

te Heesen 2000 - Anke te Heesen: Boxes in Nature. In: Studies in History and Philosophy of Science 31/3 (2000), S. 381-403. 
te Heesen \& Spary 2001 - Anke te Heesen \& Emma C. Spary (Hg.): Sammeln als Wissen. Das Sammeln und seine wissenschaftsgeschichtliche Bedeutung, Göttingen 2001.

te Heesen 2002 - Anke te Heesen: Vom naturgeschichtlichen Investor zum Staatsdiener. Sammler und Sammlungen der Gesellschaft Naturforschender Freunde zu Berlin um 1800. In: Anke te Heesen \& Emma C. Spary (Hg.): Sammeln als Wissen. Das Sammeln und seine wissenschaftsgeschichtliche Bedeutung, Göttingen 2002, S. 62-84.

te Heesen 2005 - Anke te Heesen: Accounting for the Natural World. Double-

Entry Bookkeeping in the Field. In: Londa Schiebinger \& Claudia Swan (Hg.):

Colonial Botany. Science, Commerce, and Politics in the Early Modern World, Philadelphia 2005, S. 237-251

te Heesen 2008 - Anke te Heesen: in medias res. Zur Bedeutung von

Universitätssammlungen. In: NTM Zeitschrift für Geschichte der Wissenschaften, Technik und Medizin 16 (2008), S. 485-490, DOI:

https://doi.org/10.1007/s00048-008-0312-3 (Zugriff: 22.7.2019).

te Heesen et al. 2013 - Anke te Heesen, Jochen Hennig \& Katrina Schulz (Hg.):

Kunstblätter - Blätterkunst. Objekterkundungen am Arboretum der Humboldt-Universität zu Berlin (Texte des Bachelor-Seminars „Die Sammlungen der Humboldt-Universität. Übungen am Objekt“ Q-Tutoriums „Kunstblätter - Blätterkunst“ im Wintersemester 2012/2013), Berlin 2013.

te Heesen \& Vöhringer 2014 - Anke te Heesen \& Margarete Vöhringer: »Wissenschaft im Museum - Ausstellung im Labor«, In: Anke te Heesen \& Margarete Vöhringer (Hg.): Wissenschaft im Museum - Ausstellung im Labor (LiteraturForschung, Bd. 20), Berlin 2014, S. 7-17.

Thiers 2019 - Barbara M. Thiers: The World's Herbaria 2018. A Summary

Report Based on Data from Index Herbariorum, 10.1.2019, URL: http://sweetgum.nybg.org/science/ih/annual-report/ (Zugriff: 27.7.2019)

Tütken 2005 - Johannes Tütken: Privatdozenten im Schatten der Georgia Augusta. Zur älteren Privatdozentur (1734-1831). Teil I Statutenrecht und Alltagspraxis, Göttingen 2005.

Vogel 2013 - Jakob Vogel: Public-private partnership. Das koloniale Wissen und seine Ressourcen im langen 19. Jahrhundert. Einführung. In: Rebekka Habermas \& Alexandra Przyrembel (Hg.): Von Käfern, Märkten und Menschen. Kolonialismus und Wissen in der Moderne, Göttingen 2013, S. 261-284.

Wagenitz 1982 - Gerhard Wagenitz: Index collectorum principalium herbarii Gottingensis, Göttingen 1982.

Wagenitz 1988 - Gerhard Wagenitz: Göttinger Biologen 1737-1945. Eine biographisch-bibliographische Liste (Göttinger Universitätsschriften, Bd. 2), Göttingen 1988. 
Wagenitz 1993 - Gerhard Wagenitz: Das Herbar des Systematisch-

Geobotanischen Instituts der Universität Göttingen - Archiv und

Forschungsstätte. In: Georgia Augusta 59 (1993), S. 43-53.

Wagenitz 1996 - Gerhard Wagenitz: Geobotanik in Göttingen. Von Albrecht von Haller bis Franz Firbas. In: Verhandlungen der Gesellschaft für Ökologie 25 (1996), S. 9-25.

Wagenitz 2003 - Gerhard Wagenitz: Floristik und Geobotanik in Göttingen von

Albrecht von Haller bis Heinz Ellenberg. In: Tuexenia 23 (2003), S. 41-50.

Wagenitz 2016 - Gerhard Wagenitz: Ausgewählte Objekte der Sammlung Königliche Gartenbibliothek Herrenhausen. Herrenhäuser Herbarien. In: Gottfried Wilhelm Leibniz Bibliothek (Hg.): Königliche Gartenbibliothek Herrenhausen. Eine kostbare Sammlung, ihre Geschichte und ihre Objekte (Schatzkammer 2), Hannover 2016, S. 222-232.

Weber 2011 - Cornelia Weber: Universitätssammlungen und -museen. In: Ulrich Rasche (Hg.): Quellen zur frühneuzeitlichen Universitätsgeschichte. Typen,

Bestände, Forschungsperspektiven (Wolfenbütteler Forschungen, Herausgegeben von der Herzog August Bibliothek, Bd. 128), Wiesbaden 2011, S. 83-118.

Wietschorke 2013 - Jens Wietschorke: Historische Forschung in der Europäischen Ethnologie. Ein Diskussionsbeitrag. In: Reinhard Johler et al. (Hg.): Kultur_Kultur. Denken. Forschen. Darstellen (38. Kongress der Deutschen Gesellschaft für Volkskunde in Tübingen vom 21. bis 24. September 2011), Münster et al. 2013, S. 206-212.

Winnerling 2014 - Tobias Winnerling: Das Kräuterbuch als frühneuzeitliches Gebrauchs-Objekt? In: Friederike Elias et al. (Hg.): Praxeologie. Beiträge zur interdisziplinären Reichweite praxistheoretischer Ansätze in den Geistes- und Sozialwissenschaften (Materiale Textkulturen, Schriftenreihe des Sonderforschungsbereichs 933, Bd. 3), Berlin \& Boston 2014, S. 165-197.

Wörz 2016 - Arno Wörz: Der Esslinger Botanische Reiseverein 1825-1845.

Eine Aktiengesellschaft zur Durchführung naturkundlicher Sammelreisen (Stuttgarter Beiträge zur Wissenschafts- und Technikgeschichte, Bd. 9), Berlin 2016.

Wunschmann 1971a - Ernst Wunschmann: Schrader, Heinrich Adolf. In: Allgemeine Deutsche Biographie 32 (19712), S. 429-430.

Wunschmann 1971b - Ernst Wunschmann: Bartling, Friedrich Gottlieb. In: Allgemeine Deutsche Biographie 46 (19712), S. 224-225.

Wunschmann 1883 - Ernst Wunschmann: Lantzius-Beninga, Scato. In: Allgemeine Deutsche Biographie 17 (1883), S. 702-703, URL: https:/ /www.deutsche-biographie.de/pnd116731516.html\#adbcontent (Zugriff: 28.07.2019) 
Yale 2016 - Elizabeth Yale: Introduction. "A Whole and Perfect Bodie and Book". Constructing the Human and Natural History of Britain. In: Elizabeth Yale: Sociable Knowledge. Natural History and the Nation in Early Modern Britain, Pennsylvania 2016, S. 1-19.

IV. Internetquellen

Internetauftritt der Ausstellung 38 Dinge - Schätze aus den natur- und kulturwissenschaftlichen Sammlungen der Universität Tübingen (19.5.-28.5.2006), URL: https://t1p.de/Ausstellung-38-Dinge (Zugriff: 307.2019).

Briefe von F. G. Bartling an G. Kunze in der Universitätsbibliothek Leipzig, URL: https://t1p.de/Eintrag-in-Kalliope (Zugriff: 30.7.2019).

Herbarium Göttingen,

URL: https://www.unigoettingen.de/de/herbarium+goet/157034.html (Zugriff: 25.7.2019). 


\section{$5 \quad$ Abbildungsverzeichnis}

Abb. 1:

Herbarbeleg von Lepidium densiflorum Schrad., kultiviert im botanischen Garten Göttingen, Herbarium Göttingen, GOET002629.

Abb. 2:

Verzeichnis von Veröffentlichungen von H. A. Schrader (mit Standnummern in U.B.), in denen neue Arten beschrieben wurden, mit Aufzählung einiger dieser Arten, Seite 1, Albrecht-von-Haller-Institut für Pflanzenwissenschaften, PWI.Syst.Geobotan.HE15.

Abb. 3:

Verzeichnis von Veröffentlichungen von H. A. Scbrader (mit Standnummern in U.B.), in denen neue Arten beschrieben wurden, mit Aufzäblung einiger dieser Arten, Seite 2, Albrecbt-von-Haller-Institut für Pflanzenwissenschaften, PWI.Syst.Geobotan.HE15.

Abb. 4:

Herbarbeleg von Dianthus caryophyllus L., gesammelt von F. G. Bartling im Juli 1818 nabe des Kap Kamenjak an der südlichen Spitze der Halbinsel Istrien, Herbarium Göttingen, GOET005946. 
Abb. 5:

Einem Schreiben F. G. Bartlings vom 21. Dezember 1833 an den Prorektor und den Senat der Universität beigefügte Zeichnung, Universitätsarchiv Göttingen, UniA GÖ, Kur., 6912.

Abb. 6:

Einem Schreiben Landbauinspektors Otto Prä̈l vom 24. Mär. 1833 (UniA GÖ, Kur., 6912) an das Kuratorium beigefügte Zeichnung vom Landbauconducteur Weyland, Plan von der Lage des ehemaligen Wohnhauses vom Hofrat Meister an der Paulinerstraße ₹u Göttingen, Universitätsarchiv Göttingen, UniA GÖ, Kart., 3.

Abb. 7:

Einem Schreiben des Landbauinspektors Otto Prä̈l vom 8. Januar 1834 (UniA GÖ, Kur., 6912) an das Kuratorium beigefügte Zeichnung, Grundriss vom Meisterschen Hause ₹u Göttingen, Universitätsarchiv Göttingen, UniA GÖ, Kart., 3.

Abb. 8:

Herbarbeleg von Brachycarpea juncea Marais, gesammelt von C. F. Ecklon im September 1834 auf einem Hügel bei ,Brackfontein' (Clanwilliam), Herbarium Göttingen, GOET002720.

Abb. 9:

Herbarbeleg von Vincetoxicum hirundinaria Medik., gesammelt von F. G. Bartling im September 1840 im oberen Isonzo-Tal bei Ternova (Ternova d'Isonzo/Trnova ob Soči), Herbarium Göttingen, GOET005774.

Abb. 10:

Herbarbeleg von Vincetoxicum hirundinaria Medik., gesammelt von F. G. Bartling im September 1840 im oberen Isonzo-Tal bei Ternova (Ternova d'Isonzo/Trnova ob Soči), Herbarium Göttingen, GOET005775.

Abb. 11:

Herbarbeleg von Salicornia europaea L., gesammelt von B. S. G. Lantzius-Beninga im Jahr 1847 am Meer bei Petkum (1 a-d) sowie auf der Insel Langeoog (2), Herbarium Göttingen, obne Inventarnummer.

Abb. 12:

Herbarbeleg von Tepualia stipularis (Hook. \& Arn.) Griseb., gesammelt von R. A. Philippi im Jahr 1852 in der chilenischen Provinz Cordillera, Herbarium Göttingen, GOET007539.

Abb. 13:

Herbarbeleg von Tepualia stipularis (Hook. \& Arn.) Griseb., gesammelt von R. A. Philippi im Jabr 1852 in der chilenischen Provinz Cordillera, Herbarium Göttingen, GOET007540. 
Abb. 14:

Herbarbeleg von Leontodon berinii (Bartl.) Roth, gesammelt von F. G. Bartling im September 1830 in Forojulio in der Nähe des italienischen Ortes Fogliano Redipuglia, Herbarium Göttingen, GOET001786.

Abb. 15:

Seite 87 der Vorlesungsnachschrift des Studierenden Schünemann, Wintersemester 1836/37, Vorlesung über die Organographie und Physiologie der Pflanzen von F. G. Bartling, Staats- und Universitätsbibliothek Göttingen, Handschriftenabteilung, Cod. Ms. 1990.24/1.

Abb. 16:

Seite 75 der Vorlesungsnachschrift des Studierenden W. A. Roth, Wintersemester 1836/37, Vorlesung über die Organographie und Physiologie der Pflanzen von F. G. Bartling, Staats- und Universitätsbibliothek. Göttingen, Handschriftenabteilung, Cod. Ms. 2016.12/2.

Abb. 17:

Seite 50 der Vorlesungsnachschrift des Studierenden G. Schwalbe, Sommersemester 1821, Staats- und Universitätsbibliothek. Göttingen, Handschriftenabteilung, 4 Cod. Ms. hist. nat. 63 d: 13.

Abb. 18:

Seite 25 der Vorlesungsnachschrift des Studierenden G. Schwalbe, Sommersemester 1821, Staats- und Universitätsbibliothek, Göttingen, Handschriftenabteilung, 4 Cod. Ms. hist. nat. 63 d: 13. 
n dieser kulturanthropologischen Untersuchung fragt Frauke Johanna Ahrens nach der wissens- und wissenschaftsgeschichtlichen Bedeutung der Herbarbelege des Göttinger Herbariums für den Zeitraum von 1832 bis 1852 . Anhand einer historischen, objektfokussierten Ethnografie und Praxeologie schlüsselt sie auf, wie sich die wissenskonstituierenden Praktiken und das wissenschaftliche Erkenntnisinteresse in der Etablierungsphase der 1832 institutionalisierten Sammlung wechselseitig beeinflussten und sich vor allem auch in den botanischen Präparaten niederschlugen. In fünf Kapiteln, denen je eine Praxis - die Sammlung öffnen, Werte zuschreiben, Pflanzenmaterial akquirieren, mit Herbarbelegen forschen, mit Herbarbelegen lehren gewidmet ist, und begleitet durch detaillierte Objektbeschreibungen wird so die Materialität des Wissens herausgearbeitet. 